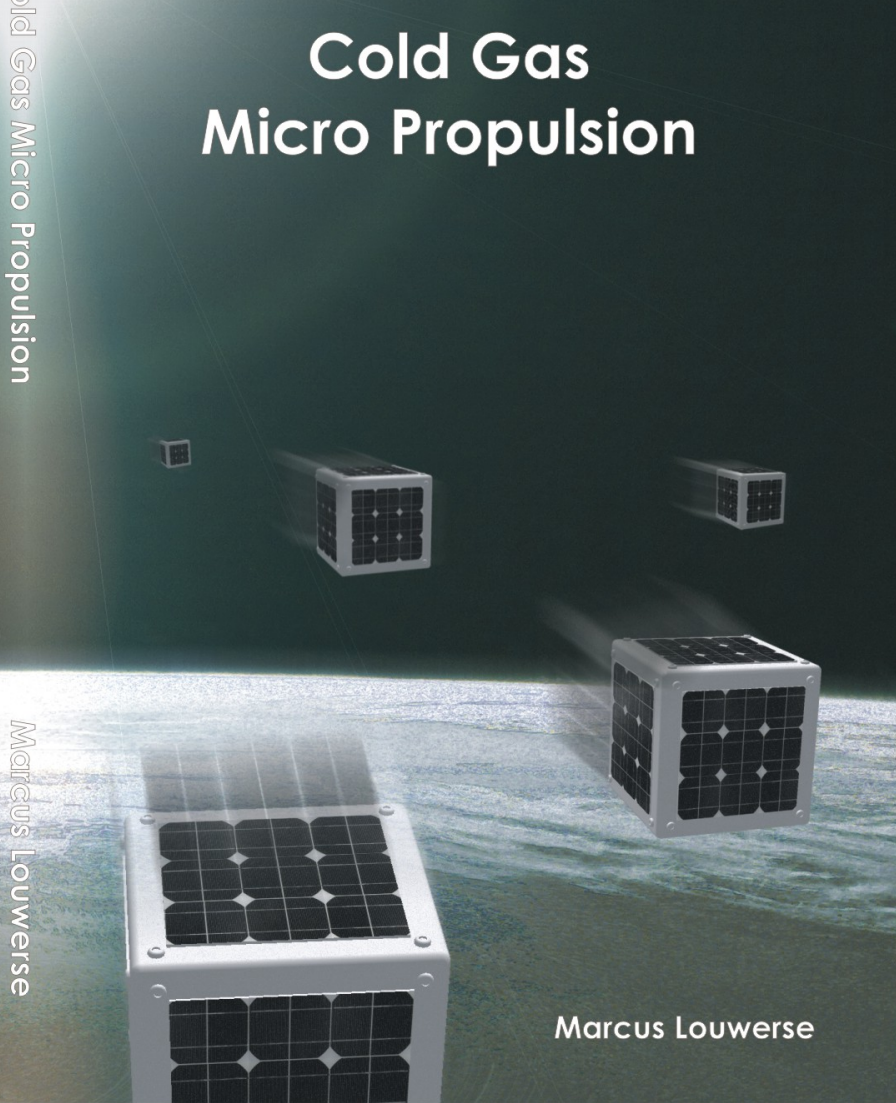

ISBN: $978-90-365-2903-7$ 


\section{Cold Gas Micro Propulsion}

Marcus Cornelis Louwerse 
The research described in this thesis was carried out at the Transducers Science and Technology Group of the MESA+ Research Institute at the University of Twente, Enschede, The Netherlands. The project was financially supported by MicroNed.

Promotiecommissie:

Voorititter

Prof. dr. ir. A.J. Mouthaan Universiteit Twente Secretaris

Prof. dr. ir. A.J. Mouthaan Universiteit Twente Promotor

Prof. dr. M.C. Elwenspoek Universiteit Twente Assistent promotor

Dr. ir. H.V. Jansen Universiteit Twente Leden

Prof. dr. L. Stenmark Universiteit Uppsala

Prof. dr. ir. A.J. Huis in 't Veld Universiteit Twente

Prof. dr. ir. H.J.M. ter Brake Universiteit Twente

Prof. dr. ir. H.W.M. Hoeijmakers Universiteit Twente

Bijzonder deskundige

Ir. H.M. Sanders

TNO Defence, security and safety

Louwerse, Marcus Cornelis

Cold gas micro propulsion

Ph.D. Thesis, University of Twente, Enschede, The Netherlands

ISBN: 978-90-365-2903-7

Copyright (C) 2009 by M.C. Louwerse, Enschede, The Netherlands 


\title{
COLD GAS MICRO PROPULSION
}

\author{
PROEFSCHRIFT
}

ter verkrijging van

de graad van doctor aan de Universiteit Twente,

op gezag van de rector magnificus,

prof. dr. H. Brinksma,

volgens het besluit van het College voor Promoties

in het openbaar te verdedigen op

vrijdag 30 oktober 2009 om 16.45 uur

door

Marcus Cornelis Louwerse

geboren op 8 december 1980

te Neerijnen 
Dit proefschrift is goedgekeurd door de promotor en de assistent promotor:

Prof. dr. M.C. Elwenspoek

Dr. ir. H.V. Jansen 


\section{Contents}

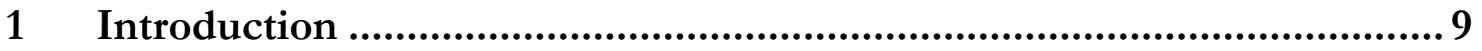

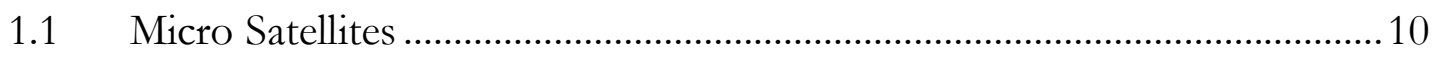

1.2 Micro propulsion systems........................................................................... 11

1.3 Problem statement and motivation .................................................................13

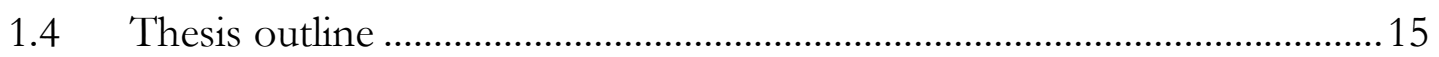

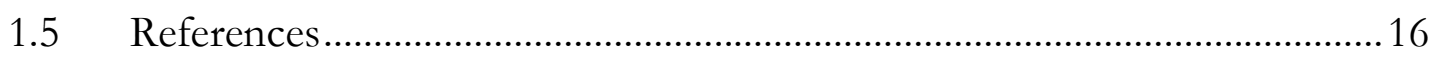

2 Cold gas propulsion: Requirements and design guidelines ....................19

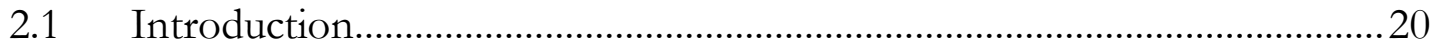

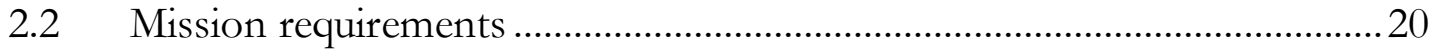

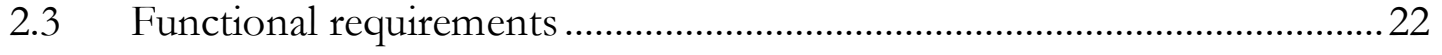

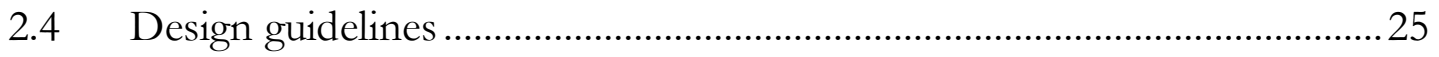

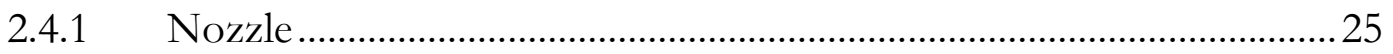

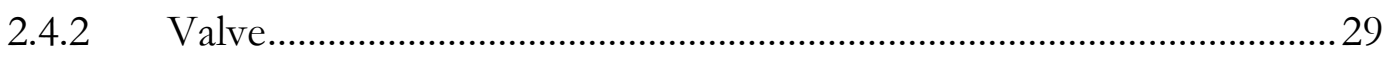

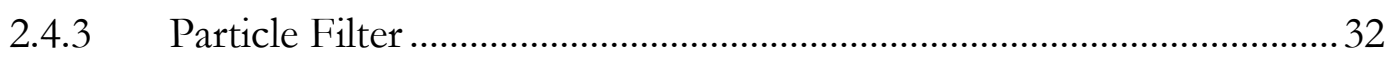

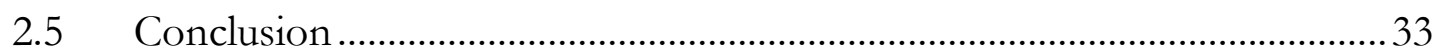

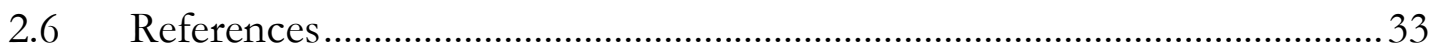

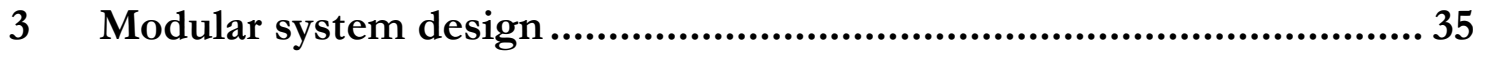

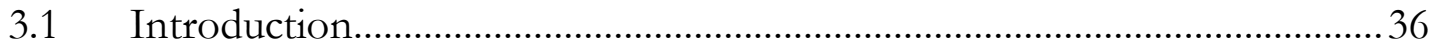

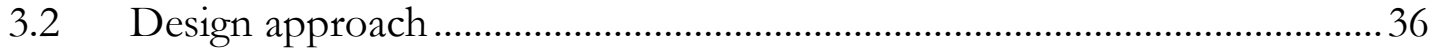

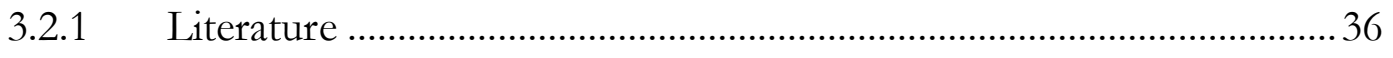

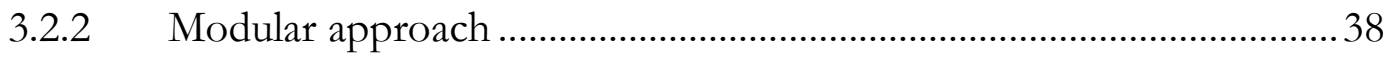

3.3 Modular feeding and thruster system.............................................................

3.3.1 Baseline glass tube package ....................................................................39

3.3.2 Functional modules ............................................................................... 44

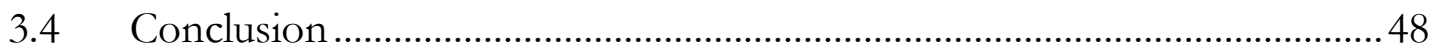

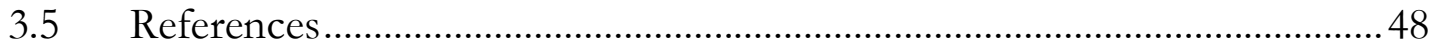

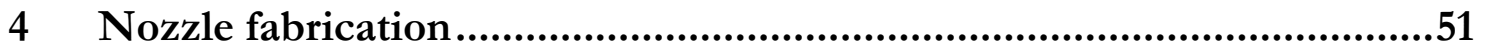

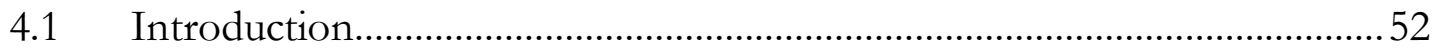

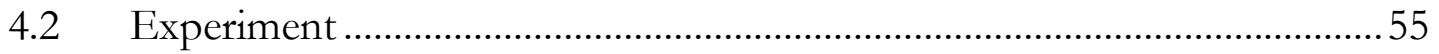

4.3 Fabrication methods......................................................................................56 
4.3.1 Deep Reactive ion etching …………................................................... 56

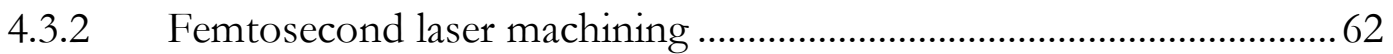

4.3.3 Powder blasting and heat treatment ....................................................... 68

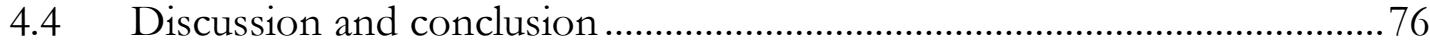

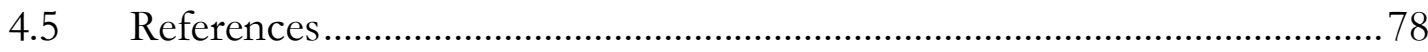

$5 \quad$ Valve for micro propulsion.............................................................81

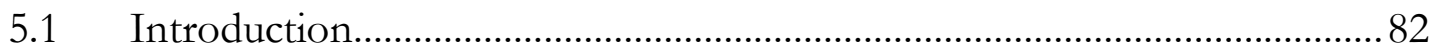

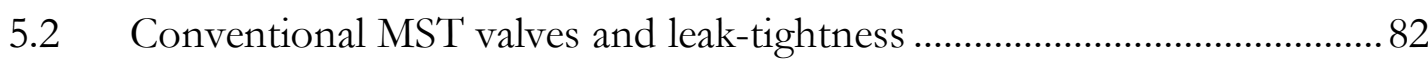

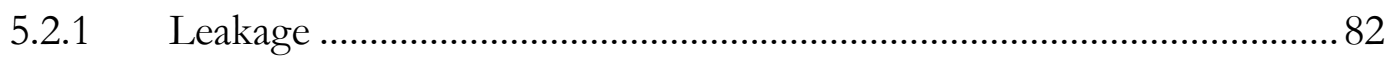

5.2.2 Micro machined valves, state-of-the-art ............................................. 87

5.3 The flexible membrane valve ....................................................................... 93

5.3.1 Functional valve design .................................................................. 93

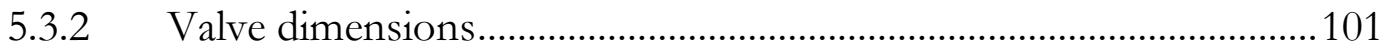

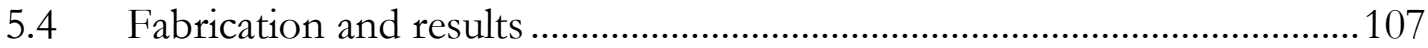

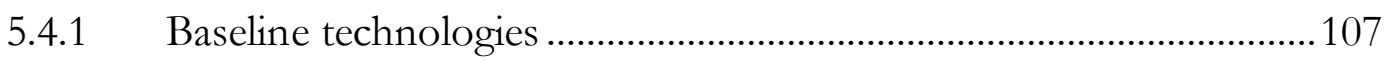

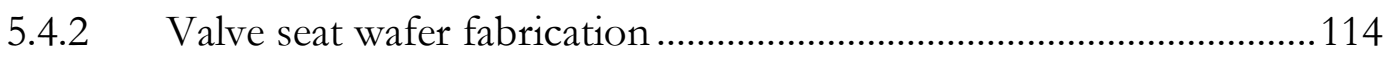

5.4.3 Membrane fabrication..................................................................... 118

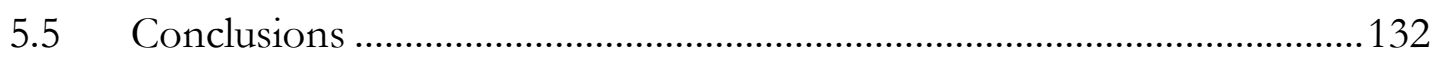

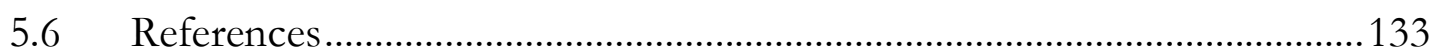

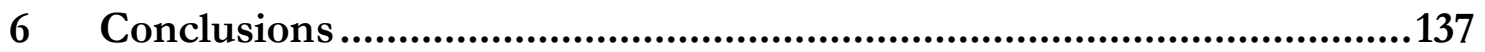

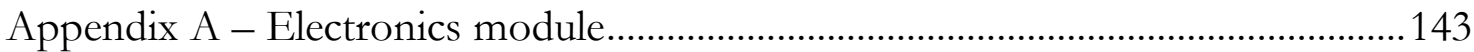

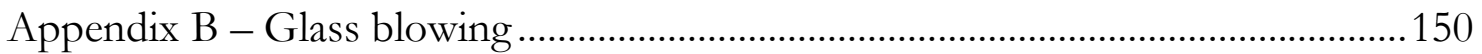

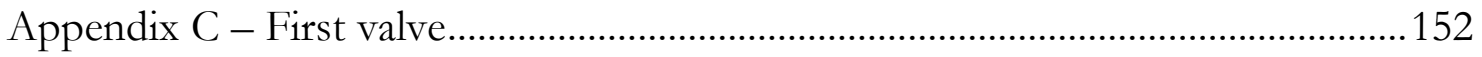

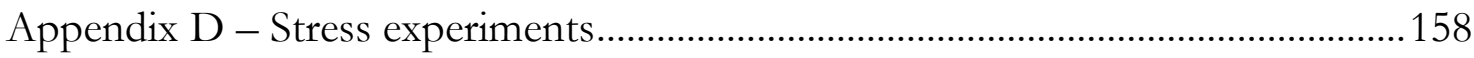

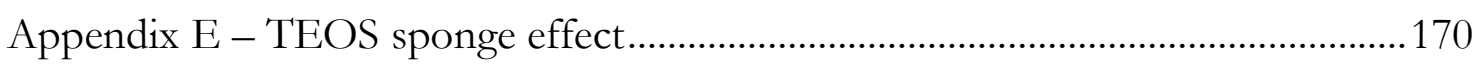

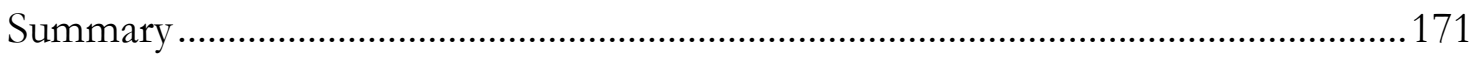

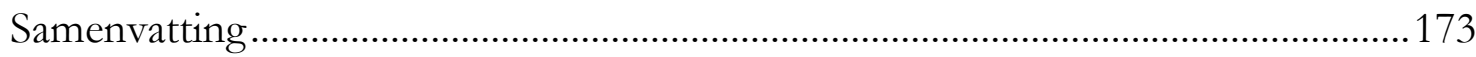

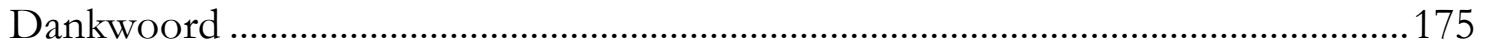






\section{1}

\section{Introduction}

This chapter gives an introduction into micro propulsion. Different types of propulsion pass the review and the motivation for further research is given. At last the outline of this thesis is discussed. 


\subsection{Micro Satellites}

In modern times satellites play a crucial role in a lot of research fields and in everyday live. Satellites are used for communication purposes, global positioning systems, whether and climate monitoring, astronomical research and more.

The high cost of large satellites is a bottleneck for universities and research centers to incorporate new technologies that not yet have proven themselves and therefore risk the mission. The advent of the miniaturization of satellites enables low cost access to space and makes alternative technologies more attractive. Besides the reduction of costs, the miniaturization of satellites has several advantages and opens up new possibilities [1]. A large complex satellite can be replaced by multiple small satellites resulting in improved reliability and flexibility. One can imagine a cluster of several miniaturized satellites working together to perform complex missions. Some applications are optical interferometry, navigation systems and high risk missions for which a large number of identical satellites is send on a mission to ensure success despite of some satellites that do not make it. These miniaturized satellites are called 'micro satellites' because their building block require dimensions down to the micrometer scale.

The research described in this thesis is done as part of the Dutch MicroNed Programme within the MISAT cluster. The aim of the MISAT cluster is to create a micro satellite platform for research and development of innovative space applicable devices based on Micro System Technology (MST). An important obstacle for the cost reduction of small satellites is the lack of a well defined platform for subsystems. At this moment every satellite is unique and subsystems are closely intertwined making it difficult to reuse parts of already designed satellites. For future satellites it is envisioned that they are designed in a more modular and plug \& play fashion.

More advanced missions require the possibility to correct the attitude and/or altitude of a satellite by means of a propulsion system. The title of this thesis, 'Cold gas micro propulsion', refers to the steering of small space vehicles by means of miniaturized propulsion systems. Propulsion is achieved by expelling small amounts of 'cold gas'. The word 'micro' not only refers to the small size of these systems but also to the method of manufacturing: Micro System Technology. 


\subsection{Micro propulsion systems}

In space, a vehicle can accelerate itself by ejecting part of its mass. This acceleration mechanism is called thrust and a device that generates thrust is called a thruster. The nozzle is a key component of the thruster because it increases the efficiency of the system. In the way the nozzle is shaped, it increases the supersonic velocity of a gaseous exhaust. With the same amount of expelled mass more thrust is generated and thus efficiency is improved. There are several methods by which thrust can be generated, not all of them are suitable for miniaturization [2]. When we focus on miniaturized propulsion systems the following types are found.

In the concept of the Resistojet, electric power is used to produce heat and thereby a mechanism is triggered to generate thrust. The generation of heat can be used to vaporize a liquid which is accelerated through a nozzle to obtain thrust. This principle is used by several research groups [3-6]. Kang uses another mechanism based on the generation of a vapor bubble. The rapid growth of a vapor bubble is used to eject a plug of liquid propellant [7]. This mechanism is widely used in inkjet printers.

Colloid thrusters have been developed which produce thrust by the electrostatic acceleration of liquid droplets forming an electrostatic spray [8, 9]. Pulsed Plasma thrusters are also explored for miniaturization. In a pulsed plasma thruster an arc is used to ablate and accelerate a small amount of solid propellant typically Teflon [10]. Hrbud explored the use of RF plasma for micro-propulsion purposes. An RF capacitive coupled discharge is heating a propellant, and thermodynamic expansion of the gas generates thrust [11].

Another type of propulsion which is emerging is the laser-driven micro-rocket. Short laser pulses are used to ablate a small amount of material and thereby obtain thrust [12-14].

In a solid propellant system an array of small chambers, each having an igniter and a nozzle, is filled with a solid material. The solid propellant decomposes when enough heat is supplied. After ignition the hot gaseous products leave the chamber 
through the nozzle. In this way a predefined amount of impulse is generated and it is therefore also called digital propulsion [15-22].

The above mentioned propulsion mechanism all use a solid material or a liquid stored at low pressure, as propellant. The following mechanisms are based on a propellant - gas or liquid - that is stored at high pressure. Besides a pressurized fuel tank such a propulsion system can consist of several components, i.e. filters, valves, pressure regulators, tubing, heaters and thrusters. For health monitoring, pressure sensors, flow meters, and temperature sensors might be necessary. Depending on the type of propulsion, this can be quite a complex system. Three different configurations of propulsion systems are discussed in order of increasing complexity and efficiency and all utilizing a pressurized gas tank.

\section{Cold gas propulsion}

Cold gas propulsion is the simplest configuration, requiring the least components. In a controlled manner gas is released from a tank by a valve and expelled through the thruster. Such a cold gas blow-down rocket engine typically consists of a gas tank, a pressure and temperature sensor, an on/off valve and a thruster. If the tank contains a gas at a very high pressure a pressure regulator is required in between the tank and the valve to reduce the pressure to a convenient operation level. Grönland applied MST to build a cold gas thruster pod [23]. Another such system is presented by Köhler [24]. Although no MST was used, Gibbon and Wart presented a miniaturized propulsion system based on the vaporization of butane [25]. Butane is stored as a liquid propellant and is vaporized at a pressure of $3.8 \mathrm{bar}$ at $40^{\circ} \mathrm{C}$. The heated vapor is expelled through the thruster.

The efficiency of a cold gas thruster can be increased by simply increasing the temperature of the exhaust. The higher the temperature of the gas entering the nozzle the more thrust is generated. In other words, less mass has to be expelled to obtain the same amount of thrust and thus efficiency is improved. In addition to the cold gas configuration a heat exchanger is required just in front of the nozzle to ensure an elevated temperature of the gas when entering the nozzle. 


\section{Mono-propellant propulsion}

For the monopropellant thruster, two types can be discriminated, the solid propellant type, as is previously mentioned, and the liquid propellant type. The latter type stores the propellant in a reservoir from which it is released by a valve. The propellant is injected into a catalyst chamber where it is chemically decomposed. Due to the exothermal nature of the chemical reaction a large amount of heat is released. The hot gasses are expelled at supersonic speed through the thruster. Besides the components required for cold gas propulsion this configuration needs a catalyst chamber and heater to decompose the gas. To get some feedback of what is happening during the chemical reaction one needs to monitor pressure and temperature. The high temperature of the gaseous products puts higher demands on the material the thruster is made of. A few monopropellant propulsion systems have recently been developed [26-28].

\section{Bi-propellant propulsion}

The bi-propellant system is the most complex configuration. It requires two propellant tanks, with separate feeding systems consisting of valves, tubing and pressure monitoring. The propellants are mixed and ignited in a combustion chamber. Compared to the monopropellant system more heat is generated and therefore the bi-propellant system is most efficient. The amount of components increases the complexity even more and the higher temperatures narrow down the variety of materials that can be used in such a harsh environment. At the Massachusetts Institute of Technology (MIT) work has been done on an ambitious project aiming at the development of a millimeter-scale gas turbine engine [29].

\subsection{Problem statement and motivation}

To enable formation flying where the distance between two micro satellites is controlled, an extremely miniaturized propulsion system is required. Conventional, commercially available, propulsion system components are too large, heavy and powerful to be used on micro satellites. Miniaturization is a driving force to explore other technologies than conventional fine machining techniques. A promising technology that can be used in the fabrication of small complex systems is MST. With MST sub-micrometer features can be made which allows a tremendous reduction in size and mass compared to fine machining techniques. Additionally, 
when MST is used in a smart way several components can be integrated in one device which makes the technology appealing for a complex propulsion system. MST plays a crucial role in the research described in this thesis. It forms a basis for the development of manufacturing processes specifically required for micro propulsion systems.

Our research is focused on propulsion systems that utilize a pressurized gas tank. This choice is mainly driven by the availability of a low pressure storage system which is developed by TNO [30]. In conventional systems such a fuel tank adds a considerable amount of mass to the system. A thick tank wall is necessary to withstand the high pressure. TNO has developed a novel cold gas generator (CGG) technology which allows for a low storage pressure and thus a reduced tank mass. A CGG contains a solid material in which a gas is chemically stored. When ignited the gas is released at low temperature. This allows for several refills of the tank and thus a low operating pressure, without compromise on the amount of propellant. Three configurations are distinguished as discussed before: cold gas, mono-propellant and bi-propellant propulsion. The latter two configurations both use a chemical reaction in which a lot of heat is generated. In the mono-propellant system a single propellant is decomposed in a catalyst chamber while for the bi-propellant system two gasses are fed into a combustion chamber where they react. The high temperatures that are reached due to the exothermal reactions complicate the design of the propulsion system. The materials on which MST is based - i.e. silicon and glass-like materials - might not be able to withstand such an environment. Since the development of our propulsion system is done from scratch we will keep it as simple as possible.

The starting point is a feeding and thruster system as part of a cold gas blow-down rocket engine; its main components being a leak-tight valve and a conical converging-diverging nozzle. These two components are crucial for any of the three mentioned propulsion types and therefore form the basis for more complex systems. Several nozzles have been made by MST although none of them have a conical converging-diverging shape which is optimal for a propulsion system. Novel fabrication methods are explored to make such a nozzle. Many valves have been developed by MST but most of them have too high leak rates which is disastrous for the lifetime of a satellite mission. A leak-tight valve is decisive for a successful mission. The miniaturization of valves is mainly bound by the size of the actuator. 
Especially when leak-tightness is neck and crop, the conventional approach requires a forceful and thus large actuator. To allow for even smaller valves a novel design is presented requiring low force while still being leak-tight. Furthermore, the emphasis of the research will be on the integration of several functional parts in a modular manner resulting in a miniaturized feeding and thruster system as part of a plug $\&$ play propulsion system.

\subsection{Thesis outline}

The second chapter deals with the mission requirements for the formation flying of two small satellites. Based on these mission requirements the functional design of a simple blow-down rocket engine is presented consisting of two parts; the propellant storage and the thruster and feeding system. The emphasis of further research will be on the latter and some design guidelines are derived.

The third chapter discusses our vision on a modular platform for complex systems based on MST. Starting with a simple glass tube package it is shown how several functional parts can be integrated to perform a complex task all together. Inside the glass tube the electronics for the valve actuation and for the pressure and temperature sensing is positioned. On top of the glass tube the valve and nozzle are attached which is the hart of the system.

The conical converging-diverging nozzle is central in chapter four. Three potential technologies are explored to make the conical converging-diverging nozzle, all with their own typical characteristics. The first method is deep reactive ion etching. For the second method femtosecond laser machining is examined. The last method describes the fabrication of a glass nozzle by means of powder blasting and a heat treatment. The fabrication methods are compared and we discuss how well they meet the requirements. Finally some thrust measurements are presented.

The fifth chapter describes the development of a leak tight valve. We point out the problems concerning leakage and discuss the difficulty of making an MST based valve which meets the leakage requirement. A novel approach for the design of a leak tight valve is considered. The fabrication process of this valve is discussed and measurements for leakage and throughput are discussed.

The final chapter gives an overall conclusion and recommendations are given for further research. Furthermore, we look at the possibilities of using the developed technology for future satellite missions requiring micro propulsion. 


\subsection{References}

[1] Janson S., Helvajian H., Amimoto S., Smit G., Mayer D. and Feuerstein S., Microtechnology for Space Systems, IEEE Aerospace Conference vol. 1, Snowmass at Aspen, CO, USA (1998), pp. 409-418.

[2] Mueller J., Thruster Options for Microspacecraft: A Review and Evaluation of State-of-the-Art and Emerging Technologies, Micropropulsion for Small Spacecraft of the Progress in Astronautics and Aeronautics series 147 (2000), p. 45.

[3] Maurya D.K., Das S. and Lahiri S. K., Silicon MEMS vaporizing liquid microthruster with internal microheater, Journal of Micromechanics and Microengineering 15 (2005), p. 966.

[4] Mukerjee E.V., Wallace A.P., Yan K.Y., Howard D.W., Smith R.L. and Collins S.D., Vaporizing liquid microthruster, Sensors and Actuators 83 (2000), pp. 231-236.

[5] Ye X. Y., Tang F., Ding H. Q. and Zhou Z. Y., Study of a vaporizing water micro-thruster, Sensors and Actuators A: Physical 89 (2001), pp. 159-165.

[6] J. Mueller, W. Tang, A. Wallace, et al., Design, Analysis and Fabrication of a Vaporizing Liquid Micro-Thruster, ALA A Paper 97-3054, Seattle, WA, USA (1997).

[7] T. G. Kang, S. W. Kim and Y.-H. Cho, High-impulse, low-power, digital microthrusters using low boiling temperature liquid propellant with high viscosity fluid plug, Sensors and Actuators A: Physical 97-98 (2002), pp. 659-664.

[8] Xiong J., Zhoua Z., Sun D. and Ye X., Development of a MEMS based colloid thruster with sandwich structure, Sensors and Actuators A 117 (2005), pp. 168172.

[9] Krpoun R., Räber M. and Shea H.R., Microfabrication and test of an integrated colloid thruster, IEEE 21st International Conference on Micro Electro Mechanical Systems (2008), pp. 964-967.

[10] Cassady R.J., Hoskins W.A., Campbell M. and Rayburn C., A Micro Pulsed Plasma Thruster (PPT) for the "Dawgstar" Spacecraft, IEEE Aerospace Conference Proceedings vol. 4, pp. 7-13.

[11] Hrbud I., Kemp G.E., Yan A.H. and Gedrimas J.G., Review of RF Plasma Thruster Development (2007). 
[12] Phipps C.R., Luke J.R., Lippert T., Hauer M. and Wokaun A., Micropropulsion using laser ablation, Appl. Phys. A 79 (2004), pp. 1385-1389.

[13] Ziemer J.K., Laser Ablation Microthruster Technology, 33rd Plasmadynamics and Lasers Conference, Maui, Hawaii (2002).

[14] Urech L., Lippert T., Phipps C.R. and Wokaun A., Polymer ablation: From fundamentals of polymer design to laser plasma thruster, Applied Surface Science $\mathbf{2 5 3}$ (2007), pp. 6409-6415.

[15] David H. Lewis Jr., Siegfried W. Janson, Ronald B. Cohen and Erik K. Antonsson, Digital micropropulsion, Sensors and Actuators 80 (2000), pp. 143-154.

[16] Zhang K.L., Chou S.K. and Ang S.S., Development of a solid propellant microthruster with chamber and nozzle etched on a wafer surface, J. Micromech. Microeng. 14 (2004), pp. 785-792.

[17] Zhang K.L., Chou S.K. and Ang S.S., Development of a low-temperature co-fired ceramic solid propellant microthruster, J. Micromech. Microeng. 15 (2005), pp. 944-952.

[18] Rossi C., Do Conto T., Esteve D. and Larangot B., Design, fabrication and modelling of MEMS-based microthrusters for space application, Smart Mater. Struct. 10 (2001), pp. 1156-1162.

[19] Rossi C., Orieuxa S., Larangota B., Do Contoa T. and Esteve D., Design, fabrication and modeling of solid propellant microrocket-application to micropropulsion, Sensors and Actuators A 99 (2002), pp. 125-133.

[20] Zhang K.L., Chou S.K. and Ang S.S., MEMS-Based Solid Propellant Microthruster Design, Simulation, Fabrication, and Testing, J. of Microelectromechanical Systems 13 (2004).

[21] Youngner D.W., Son Thai Lu, Choueiri E., et al., MEMS Mega-pixel Microthruster Arrays for Small Satellite Stationkeeping, 14th ALAA/USU Small Satellite Conference, North Logan, UT (2000).

[22] Chaalane A., Rossi C. and Esteve D., The formulation and testing of new solid propellant mixture (DB $+\mathrm{x} \% \mathrm{BP}$ ) for a new MEMS-based microthruster, Sensors and Actuators A 138 (2007), pp. 161-166. 
[23] Grönland T., Rangsten P., Nese M. and Lang M., Miniaturization of components and systems for space using MEMS-technology, Acta Astronautica 61 (2007), pp. $228-233$.

[24] Köhler J., Bejhed J., Kratz H., et al., A hybrid cold gas microthruster system for spacecraft, Sensors and Actuators A: Physical 97-98 (2002), pp. 587-598.

[25] Gibbon D. and Ward J., The Design, Development and Testing of a Propulsion System for the SNAP-1 Nanosatellite, 14th Annual/ USU Conference on Small Satellites (2000).

[26] Hebden R., Bielby R., Baker A., et al., The development and test of a hydrogen peroxide monopropellant microrocket engine using MEMS technology, 5 Round Table on Micro/Nano Technologies for Space, ESTEC/ESA, Rijswijk, The Netherlands (2005).

[27] Hitt D.L., Zakrzwski C.M. and Thomas M.A., MEMS-based satellite micropropulsion via catalyzed hydrogen peroxide decomposition, Smart Mater. Struct. 10 (2001), pp. 1163-1175.

[28] Chih-Penh Chen, Yei-Chin Chao, Chih-Yung Wu and Jungh-Chang Lee, Development of a catalytic hydrogen micro-propulsion system, Combust. Sci. and Tech. 178 (2006), pp. 2039-2060.

[29] Epstein A.H., Millimeter-Scale, Micro-Electro-Mechanical Systems Gas Turbine Engines, J. Eng. Gas Turbines Power 126 (2004), pp. 205-222.

[30] Rackemann N. J., Sanders H. M. and van Vliet L. D., Design and development of a propulsion system for a cubesat - Based on solid propellant cool gas generator technology -, ALA A 57th International Astronautical Congress, $L A C$ vol. 5 (2006), pp. 3434-3442. 


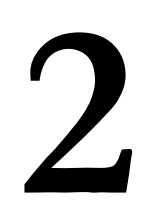

\section{Cold gas propulsion:} Requirements and design guidelines

In this chapter the requirements for a cold gas micro propulsion system are specified. These specifications are based on a mission projected to do formation flying of two micro satellites, i.e. the distance between the satellites is accurately controlled. Based on these mission requirements some design guidelines are derived for a thruster and feeding system. 


\subsection{Introduction}

Depending on the nature of the mission of a satellite, or cluster of satellites, the demands on a propulsion system vary quite a lot. For large formations of many satellites, it is envisioned that they have the capability to maintain their formation autonomously and perform complex tasks together. This requires sensors on every satellite to determine the relative distance between them. But more importantly, complex algorithms are required to determine the influence of individual position correction of satellites on the formation. It is like a swarm of sparrows flying in a continuously changing formation. For now, we focus on the formation flying of two satellites, where the distance between the satellites is maintained.

In paragraph 2.2 some mission requirements are derived for the formation flying of two satellites. The functional requirements of a cold gas propulsion system are presented in paragraph 2.3. In paragraph 2.4 the design guidelines and dimensions of individual components are derived.

\subsection{Mission requirements}

In this paragraph some mission requirements are derived that form the basis for the functional design of a cold gas propulsion system. The formation flying of two satellites implies the following; the initial relative position and velocity is maintained while the orbital altitude of the satellites may degrade. The decay of the satellites is influenced by atmospheric drag and by the geo-potential field, the Earth's gravity field. Due to differences in position, area and mass of the satellites they drift apart and for this the propulsion system is adjusting [1]. Depending on the distance the satellites are allowed to drift apart, the frequency and accuracy of the corrections are obtained. These parameters can be translated into a minimum impulse bit. For formation flying the impulse bit is simply defined by:

$$
I_{b}=m \Delta v=T t_{\min }
$$

Where $\Delta v($ delta-v) is the change in velocity and $m$ the mass of the satellite. The impulse bit is also expressed as the force $T$, which is actually the thrust, times $t_{\min }$; the smallest time unit that the thrust is delivered. When accurate positioning is important one needs a small impulse bit and a high repetition rate for the corrections. By taking the duration of the mission into account, the net velocity 
change, which is the sum of all the changes in velocity required for a particular mission, can be calculated. The total delta- $\mathrm{v}$ is then calculated by:

$$
\Delta v_{t}=\frac{\text { mission duration }}{\text { period between corrections }} \Delta v \quad[\mathrm{~m} / \mathrm{s}]
$$

When the total delta- $\mathrm{v}$ is known it is possible to calculate the amount of reaction mass which is required by using Tsiolkovsky's rocket equation [2]:

$$
\Delta v_{t}=v_{e} \ln \frac{m_{i}}{m_{f}}
$$

Where $v_{e}$ is the velocity of the reaction mass when it leaves the satellite, $m_{i}$ the initial mass and $m_{f}$ the final mass. By rewriting equation (2.3), the total amount of reaction mass for the mission is obtained:

$$
\Delta m=m_{i}-m_{f}=m_{i}-\left[m_{i} \cdot e^{-\Delta v_{t} / v_{e}}\right]
$$

A standard for micro satellites is the Cubesat which measures $10 \times 10 \times 10 \mathrm{~cm}^{3}$ and has a mass of $1 \mathrm{~kg}$ [3]. The requirements for a propulsion system used to do formation flying of two Cubesats were derived by TNO [4]. Some assumptions for the mission are given in Table 2-I. The micro propulsion system should be smaller than $10 \%$ of the satellite volume and less than $10 \%$ of the satellite mass which is $100 \mathrm{~cm}^{3}$ and 100 gram, respectively. The distance between the Cubesats is in the order of tens of kilometers and this distance should be maintained within a 2 meters margin.

For a particular orbital altitude it is calculated how fast two satellites drift apart due to small differences in area and mass [1]. To compensate for the drifting and to maintain the distance between the two Cubesats within the margin of 2 meter, 6000 corrections by the thruster are required per year. Per correction a certain amount of impulse is needed to stay within the margin. This impulse bit is $90 \mu \mathrm{Ns}$ and the total delta- $\mathrm{v}$ that is then demanded for a 1 year mission is $0.54 \mathrm{~m} / \mathrm{s}$. When assuming a supersonic exhaust of Mach 4 , a total amount of 0.8 gram nitrogen of reaction mass is needed to fulfill the mission. These requirements are summarized in Table 2-II and give a starting point for the functional design. 


\begin{tabular}{|l|l|}
\hline Mission duration & 1 year \\
\hline Orbital altitude & $500 \mathrm{~km}$ \\
\hline Satellite mass / dimensions & $1 \mathrm{~kg} / 10 \times 10 \times 10 \mathrm{~cm}^{3}$ \\
\hline Allowed drift in distance & $2 \mathrm{~m}$ \\
\hline
\end{tabular}

Table 2-I: Mission characteristics

\begin{tabular}{|l|l|}
\hline Impulse bit & $90 \mu \mathrm{Ns}$ \\
\hline Number of corrections & 6000 \\
\hline Total delta-v & $0.54 \mathrm{~m} / \mathrm{s}$ \\
\hline Assumed exit velocity & $673 \mathrm{~m} / \mathrm{s}(\mathrm{Ma}=4)$ \\
\hline Reaction mass & $0.8 \mathrm{~g}$ \\
\hline
\end{tabular}

Table 2-II: Propulsion requirements

\subsection{Functional requirements}

Here, the functional requirements of a cold gas propulsion system are presented. Our micro propulsion system is a simple blow down system of a rocket engine consisting of a thruster and feeding system and a propellant storage tank. Besides the use of micro system technology to reduce the size of the thruster and feeding system, a novel technology has been developed which makes it possible to reduce the size as well as the storage pressure of the propellant storage drastically [5]. Cold gas generators (CGG) are used which contain a gas that is chemically stored inside a solid material. When ignited, nitrogen gas is released into a gas storage tank. Several CGG's are connected to the storage tank to refill the tank when the pressure drops below a proper operation pressure for the thruster.

A functional lay-out of the propulsion system is shown in Figure 2-1. It consists of two main parts, the propellant storage and the thruster and feeding system. The propellant storage consists of a plenum with 8 cold gas generators and is developed by TNO [6]. The thruster and feeding system, consists of a valve, a particle filter, a pressure and temperature sensor and a nozzle. 


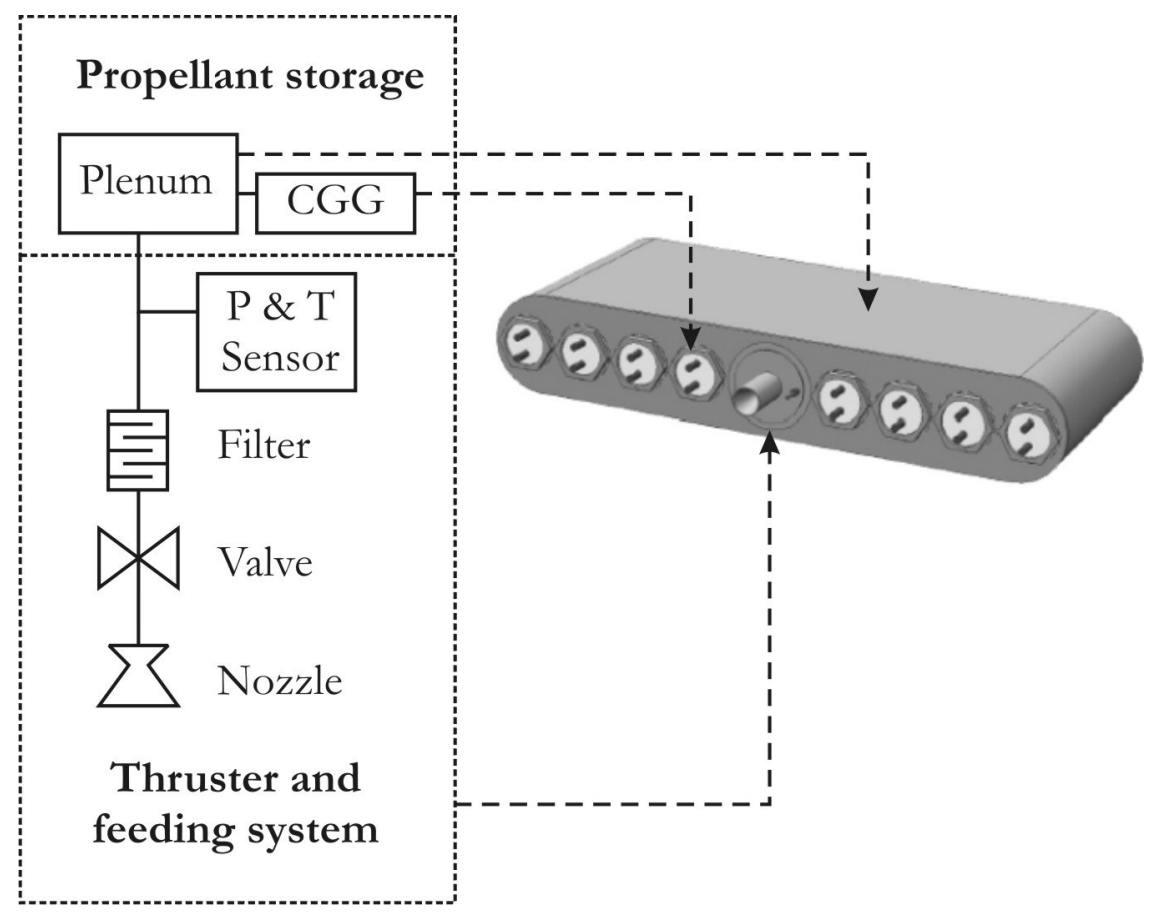

Figure 2-1: Functional lay-out of the micro propulsion system

The low storage pressure - which is enabled by the cool gas generator technology and is typically between 1 and 4.5 bar - makes a pressure regulator redundant. Such a regulator is normally required when using high-pressure tanks. Moreover, the low pressure makes storage easier and makes the design of the MST thruster and feeding system less critical in terms of fracture. To prevent system failure, if by accident more than one CGG is ignited and thus causing a high tank pressure, the system has to be able to withstand a pressure of $10 \mathrm{bar}$.

The impulse bit of $90 \mu \mathrm{Ns}$ can be achieved by a nozzle that generates a thrust in the order of 1 to $10 \mathrm{mN}$ in combination with a valve open time of 90 to $9 \mathrm{~ms}$. The time it takes to open or close the valve should be less than $1 \mathrm{~ms}$ to guarantee a precise impulse bit. This normally closed active valve has to be leak-tight for a pressure between 1 and 10 bar. A total amount of 0.8 gram nitrogen is carried by the 8 cold gas generators. Less than 0.1 gram loss of nitrogen per year due to leakage is allowed which means a valve leakage of less than $1.6 \cdot 10^{-4} \mathrm{sccm}$ (standard cubic centimeters per minute). The leakage depends on the tank pressure, since a higher tank pressure results in a faster depletion of propellant. We assume a linear relation between the tank pressure and the leak rate. When assuming a mean tank pressure of 3 bar - which is a considerable over-estimation - the maximum allowed leakage is 
$5.3 \cdot 10^{-5} \mathrm{sccm}$. To avoid leakage induced by particles getting stuck in the valve a particle filter is necessary.

To be able to accurately steer the satellite two parameters should be known:

1. Tank pressure

Our propulsion system is a blow down system meaning the pressure decreases during propulsion. The tank pressure is in between 1 and 4.5 bar depending on the amount of gas in the tank. The pressure is maintained within this range by releasing nitrogen from a CGG when the operation pressure drops below 1 bar.

2. Gas temperature

We are building a cold gas propulsion system without any thermal housekeeping. This means the gas temperature is mainly depending on the temperature of the satellite and on the heat generated by the ignition of a CGG. When the satellite is orbiting the earth, it is for part of the time in eclipse and otherwise in direct sun light. This causes periodic temperature changes of the satellite.

The tank pressure and temperature are measured to be able to determine the amount of thrust before the actual propulsion is performed. The impulse bit, which is the parameter that should be precisely controlled to be able to do formation flying, is then tuned by the duration of the thrust action as described in equation (2.1). The duration of propulsion is controlled by the valve. The measurement of pressure is also important to detect leakage and to determine when a cold gas generator has to be ignited to refill the plenum.

An important issue to be considered is the harsh environment in space and difficult launch conditions which the system has to be able to survive. First of all the system has to withstand the vibrations during the launch which requires a mechanically very stable design. When the satellite is in orbit two other environmental aspects are important namely, temperature and radiation. The operating range of the temperature depends on the thermal management of the satellite. In the extreme case the temperature can change $100^{\circ} \mathrm{C}$ from $-40^{\circ} \mathrm{C}$ to $60^{\circ} \mathrm{C}$ in 90 minutes [7]. The exposure to radiation is mainly important for sensitive electronic component which degrade over time due to radiation. But also mechanically, the protective body of the propulsion system should be thick enough to prevent puncture, and thus leakage, due to the impact of high-energy cosmic rays [8]. 


\subsection{Design guidelines}

Before making a detailed design for the hardware we look at some important dimensions of the nozzle, the valve and filter. For the propulsion system to work properly, the characteristic dimensions of these functional parts have to be matched. Some design rules are derived for the thruster and feeding system which will be used in the refined designs covered in chapter 4 and 5 .

\subsubsection{Nozzle}

To every action there is an equal and opposite reaction. This is Newton's third law of motion and governs the mechanism by which cold gas propulsion works. A satellite accelerates itself by expelling part of its mass, the reaction mass, in the opposite direction. The propulsion system accelerates or slows down the satellite.

The reaction mass is expelled through a nozzle. In a propulsion system a nozzle is used to increase the velocity of the exhaust. The higher the effective outlet velocity the more thrust is generated with the same amount of expelled mass and therefore efficiency is increased. The typical shape of a converging-diverging nozzle, or 'de Laval' nozzle, named after its inventor, is shown in Figure 2-2. At the inlet of the nozzle the gas is at a high pressure and low velocity. The flow enters the converging part of the nozzle and reaches the speed of sound in the throat of the nozzle. The gas is further accelerated in the diverging part of the nozzle till it is expelled through the nozzle exit at supersonic speed and low pressure.

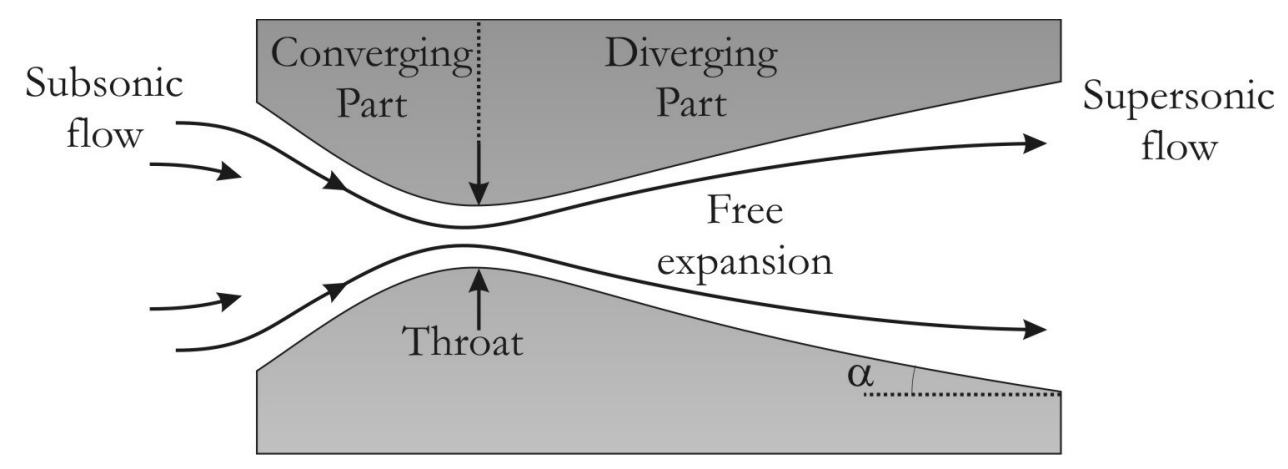

Figure 2-2: Typical nozzle geometry 
The amount of thrust that is produced depends on the mass flow rate $\dot{m}_{n}$, the exit velocity of the exhaust $v_{e}$ and the pressure at the nozzle exit $p_{e}$.

The thrust that is produced by a nozzle is calculated by [2]:

$$
T=\dot{m}_{n} v_{e}+\left(p_{e}-p_{0}\right) A_{e}
$$

Where, $p_{0}$ is the free stream pressure which is considered zero in space and $A_{e}$ is the nozzle outlet area. All of the variables in equation (2.5) depend on the dimensions of the nozzle. The equations describing the supersonic isentropic flow of an ideal gas through a converging-diverging duct are derived in [9].

The mass flow through the nozzle is mainly determined by the throat area $A_{t}$ and tank pressure $p_{n}$ and can be calculated by:

$$
\dot{m}_{n}=\sqrt{k}\left(\frac{2}{k+1}\right)^{\frac{k+1}{2(k-1)}} \frac{p_{n}}{\sqrt{R T_{c}}} A_{t}=0.0023 p_{n} A_{t} \quad[\mathrm{~kg} / \mathrm{s}]
$$

In equation (2.6) $k$ is the specific heat ratio which is 1.4 for nitrogen, $R$ is the gas constant and $T_{c}$ is the temperature of the gas in the storage tank, which we assume to be $296 \mathrm{~K}$. The exit pressure and exit velocity are related to the Mach-number at the nozzle exit. The Mach-number $(M a)$ is determined by the expansion ratio of the nozzle; i.e. the ratio between the throat area and the exit area. They are related by:

$$
\frac{A_{e}}{A_{t}}=\frac{1}{M a}\left\{\frac{1+[(k-1) / 2] M a^{2}}{1+[(k-1) / 2]}\right\}^{\frac{k+1}{[2(k-1)]}}=\frac{1}{M a}\left\{\frac{1+0.2 M a^{2}}{1.2}\right\}^{3}
$$

For a given expansion ratio the Mach-number is obtained and the ratio between the exit- and tank pressure can be calculated:

$$
\frac{p_{e}}{p_{c}}=\left\{\frac{1}{1+[(k-1) / 2] M a^{2}}\right\}^{\frac{k}{k-1}}=\left\{\frac{1}{1+0.2 M a^{2}}\right\}^{3.5}
$$

Subsequently the exit velocity is found by:

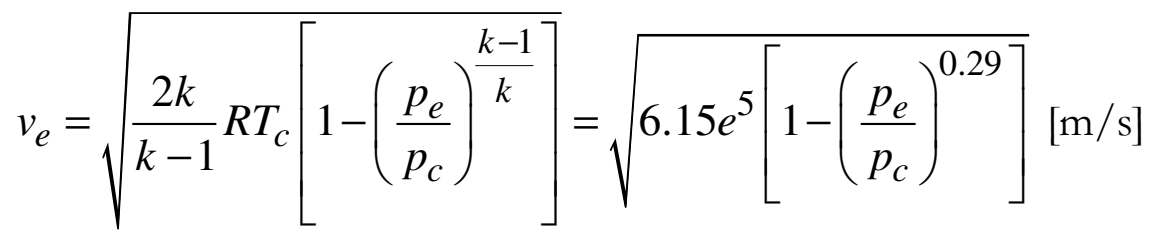

When analyzing the equations one can observe the following trend. With increasing expansion ratio the Mach-number increases and thus the exit pressure decreases and exit velocity increases. The bigger the expansion ratio the more potential energy 
is converted from gas pressure into velocity. The largest contribution to the thrust then comes from the first term of equation (2.5) which is the term responsible for increased efficiency; the faster the gas is leaving the exhaust, less mass is required to get the same amount of thrust. The second term compensates for the residual pressure working at the nozzle exit plane. Theoretically, the ideal situation would be an expansion ratio going to infinity, leaving no residual pressure. In practice this is not possible because it requires an infinitely small throat area or infinitely long nozzle to obtain an exit area reaching infinite dimension.

As is pointed out in chapter 1, the temperature of the gas at the entrance of the nozzle influences the efficiency. This tendency becomes apparent when looking at equation (2.6) and (2.9). From the first equation it is observed that the mass flow decreases when the temperature of the gas which is entering the nozzle is increased. The latter equation shows an increase in exit velocity when the gas temperature is increased. This explains the increase in efficiency when the gas is heated before entering the nozzle. With a higher temperature less reaction mass is required to obtain the same amount of thrust.

The shape of the nozzle is a static parameter; not changing during the mission. The nozzle dimensions should be chosen in such a way that it will deliver 1 to $10 \mathrm{mN}$ thrust, depending on the pressure. For an indication of the dimensions of the nozzle we assume a temperature of $20^{\circ} \mathrm{C}$ and neglect temperature influences due to the sun and CGG ignition.

To determine the dimensions of the nozzle the following assumptions are made:

- The diverging part of the nozzle has a half angle (a) in between $15^{\circ}$ and $20^{\circ}$ as depicted in Figure 2-2. A larger angle can cause rupture of the boundary layer and can result in shockwaves reducing the accuracy significantly.

- The converging part of the nozzle has a half angle of $30^{\circ}$. The converging part guides the gas towards the nozzle throat where it reaches Mach 1. Abrupt changes in the geometry should be avoided since it causes the thickness of the boundary layer to increase, and thereby reducing efficiency.

- MST is based on techniques to shape silicon and glass substrates. Since we are using this technology it is convenient to use these materials as base material for 
the nozzle. These substrates come in different thicknesses but the most common thickness is $525 \mu \mathrm{m}$ for silicon and $500 \mu \mathrm{m}$ for Pyrex glass. This dimension determines the total length of the nozzle as is further discussed in Chapter 4. We assume a converging length of $100 \mu \mathrm{m}$ and a diverging length of $400 \mu \mathrm{m}$.

Equations (2.5) till (2.9) are used to obtain some design guidelines for the dimensions of the nozzle in order to meet the requirements for the mission. Table 2-III shows the upper and lower limits of the nozzle dimensions. They are obtained with the above mentioned assumptions and the following constraints.

- Upper limit constraints:

- Maximum thrust $10 \mathrm{mN}$

- A diverging angle of $20^{\circ}$ to obtain the optimum expansion ratio resulting in Mach 4.

- Lower limit constraints:

- Minimum thrust $1 \mathrm{mN}$

- A diverging angle of $15^{\circ}$ resulting in Mach 4.1.

\begin{tabular}{|l|l|l|}
\hline & $\begin{array}{l}\text { Upper limit } \\
\mathbf{2 0 ^ { \circ }} \text { half angle }\end{array}$ & $\begin{array}{l}\text { Lower limit } \\
\mathbf{1 5 ^ { \circ }} \text { half angle }\end{array}$ \\
\hline Throat diameter $\left(d_{\text {tbroat }}\right)$ & $131 \mu \mathrm{m}$ & $90 \mu \mathrm{m}$ \\
\hline Exit diameter & $422 \mu \mathrm{m}$ & $304 \mu \mathrm{m}$ \\
\hline Thrust@ 4.5bar & $\mathbf{1 0} \mathbf{m N}$ & $4.72 \mathrm{mN}$ \\
\hline Thrust@1bar & $2.2 \mathrm{mN}$ & $\mathbf{1 . 0} \mathrm{mN}$ \\
\hline Expansion ratio & 10.4 & 11.4 \\
\hline Mach-number & 4 & 4.1 \\
\hline
\end{tabular}

Table 2-III: The upper and lower limits for the dimensions of the nozzle are calculated. The diverging length of the noz:le is assumed to be $400 \mu \mathrm{m}$ and the parameters in bold are constraint. 


\subsubsection{Valve}

An active normally closed valve is required to be able to accurately control the amount of propulsion. There are three main requirements that should be met:

1. The leakage of the valve in the closed state should be smaller than $1.6 \cdot 10^{-4} \mathrm{sccm}$ as is earlier addressed. An in depth discussion about the leakage can be found in chapter 5.

2. In the open state the area of passage should be at least 3 times higher than the nozzle throat area to avoid choked flow in the valve instead of in the nozzle.

3. The valve should open and close within $1 \mathrm{~ms}$.

Figure 2-3 shows a typical geometry of a MST valve. The static part of the valve consists of a valve seat while the active valve sealing can be used to open and close the valve. A circumferential gas flow is converging towards the valve outlet where it can enter the nozzle as is indicated by the dashed lines in Figure 2-1.

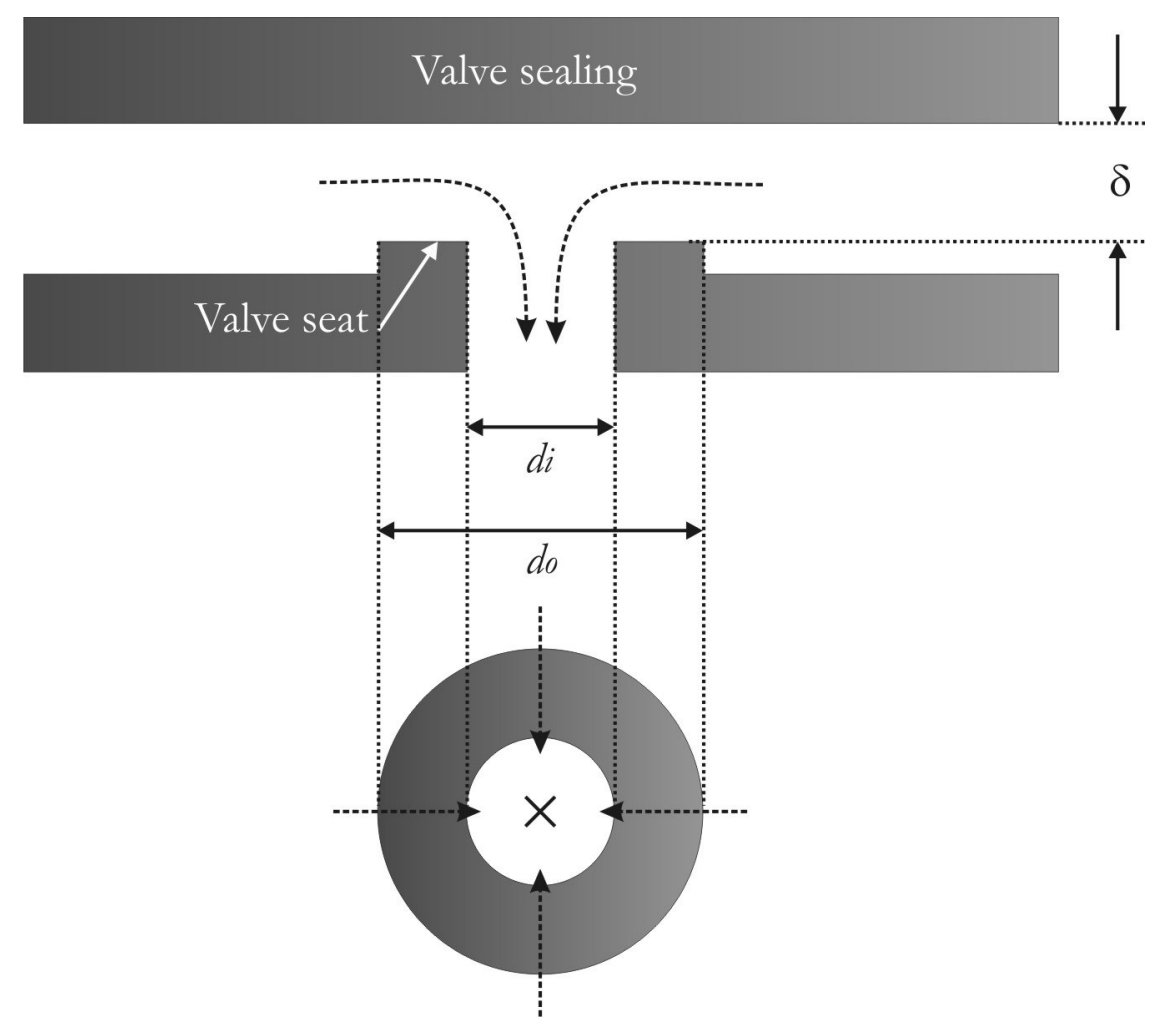

Figure 2-3: Typical geometry of a micro valve 
Assuming the inner diameter of the valve seat - indicated by $d_{i}$ in Figure 2-3 - to be much bigger than the deflection $\delta$ in the open state, the smallest area of passage of the valve can be calculated by:

$$
A_{\text {valve }}=\pi d_{i} \delta
$$

To avoid a choked flow in the valve the following constraint is considered:

$$
\begin{array}{cc}
3 A_{t}<A_{\text {value }} & {\left[\mathrm{m}^{2}\right]} \\
\frac{3}{4} \pi d_{\text {tbroat }}^{2}<\pi d_{i g} g & {\left[\mathrm{~m}^{2}\right]}
\end{array}
$$

Where $A_{t}$ is the throat area of the nozzle and $d_{t b r a t}$ the throat diameter. The upper limit for the throat diameter of the nozzle is $131 \mu \mathrm{m}$ resulting in the following guideline for the valve dimensions:

$$
d_{i} g>1.3 \cdot 10^{-8}
$$

The deflection $\delta$ of the valve sealing is determined by the actuator. When actuators are considered, four characteristics are of importance, namely; force, stroke, speed and power consumption. Common principles of actuation are piezoelectric, thermo-pneumatic, electrostatic and electromagnetic. A quick comparison of the characteristics of these actuation principles shows that piezoelectric actuation is most suitable for our application [10,11]. The thermo-pneumatic principle is discarded by the fact that it requires quite a lot of power and the operation frequency is too low. Electromagnetic actuation has a power consumption which is too high. The electrostatic method can be used at high frequencies and uses very less power but does not have a very large stroke.

In general, the force and stroke of piezo-actuators are strongly related to the size of the actuator. The total size of functional devices requiring a large force and/or stroke are mainly determined by the size of the actuator. When pursuing miniaturization, this means that the leak-tight valve should be designed in such a way that it does not require much force and stroke. In chapter 5 this point is explained in depth.

We use a piezo-disc actuator which is commercially available [12]. It has a specified free deflection of $19.1 \mu \mathrm{m}$ or a blocking force of $2.4 \mathrm{~N}$ at zero deflection when $180 \mathrm{~V}$ is applied. The diameter of the piezo-disc actuator is $12.7 \mathrm{~mm}$ and the thickness measures $410 \mu \mathrm{m}$. The Curie temperature of the piezoelectric material is $350^{\circ} \mathrm{C}$, 
meaning the material holds its piezoelectric properties up to this temperature. In the temperature range of $-40^{\circ} \mathrm{C}$ to $60^{\circ} \mathrm{C}$ the deflection of the disc deviates by $\pm 5 \%$ of the specified deflection. This deviation in deflection causes small differences in mass flow through the valve and results in a deviation in thrust. To be able to calculate an accurate impulse bit it is important to know the temperature to correct for this deviation. This piezo-actuator is fast enough to open and close the valve well within $1 \mathrm{~ms}$ which enables the small impulse bit that is required for formation flying. A disadvantage is the high voltage that is required to actuate the piezo-disc which is not standard available on a micro-satellite and so additional electronics is necessary to boost the onboard voltage.

The required force to open the valve depends on the design of the valve which is discussed in chapter 5. Preferably, the required force should be less $0.3 \mathrm{~N}$ which would result in a deflection of $16.8 \mu \mathrm{m}$. By using equation (2.13) an outlet diameter of $773 \mu \mathrm{m}$ would then be safe for the upper limit of the nozzle throat diameter. For smaller throat diameters the outlet diameter can be reduced.

To calculate the viscous mass flow through the valve following equation is used [13]:

$$
\dot{m}_{v}=\frac{\pi \delta^{3}}{12 \mu \mathrm{R} T} \frac{p_{t}^{2}-p_{n}^{2}}{\ln \left(d_{o} / d_{i}\right)}
$$

Where $\mu$ is the fluid viscosity, $R$ is the gas constant, $T$ the temperature and $p_{t}$ and $p_{n}$ the tank pressure and pressure in front of the nozzle, respectively. The geometry is defined by $\delta$ the gap height and $d_{i}$ and $d_{0}$ are the inner and outer diameter of the valve seat, respectively. To give an indication of the pressure drop over the valve when it is placed in series with the nozzle - formulas (2.6) and (2.14) are used. With the assumption that - in the steady state - the mass flow through the valve is equal to the mass flow through the nozzle, the pressure in front of the nozzle is found by solving the equations in an iterative manner. In Table 2-IV and Table 2-V the calculated pressure loss over the valve is given when it is placed in series with the nozzle. For a gap height of $16.8 \mu \mathrm{m}$ and a pressure of $4.5 \mathrm{bar}$ the Knudsen number is 0.05 which means the flow is viscous. Thus the use of equation (2.14) is justified. The Mach number in the valve is smaller than 0.3 which means compressive effects are small. 


\begin{tabular}{|l|l|}
\hline & Upper limit \\
\hline Throat diameter $\left(d_{\text {tbroat }}\right)$ & $\mathbf{1 3 1} \mu \mathrm{m}$ \\
\hline Inner diameter of valve seat $d_{i}$ & $773 \mu \mathrm{m}$ \\
\hline Valve Mach number @ 4.5bar & 0.24 \\
\hline Valve Mach number @ 1bar & 0.10 \\
\hline Pressure loss@ @ 4.5bar & 0.18 bar $(4.0 \%)$ \\
\hline Pressure loss@ 1bar & 0.17 bar $(16.5 \%)$ \\
\hline Thrust@4.5bar & $9.4 \mathrm{mN}$ \\
\hline Thrust@1bar & $1.7 \mathrm{mN}$ \\
\hline
\end{tabular}

Table 2-IV: The upper limits for the dimensions of the valve are calculated. A deflection of $17.5 \mu m$ is assumed and the difference between the inner and outer diameter of the valve seat is assumed to be $600 \mu \mathrm{m}$.

\begin{tabular}{|c|c|}
\hline & Lower limit \\
\hline Throat diameter $\left(d_{t b r o a t}\right)$ & $90 \mu \mathrm{m}$ \\
\hline Inner diameter of valve seat $d_{i}$ & $347 \mu \mathrm{m}$ \\
\hline Valve Mach number@4.5bar & 0.26 \\
\hline Valve Mach number@1bar & 0.11 \\
\hline Pressureloss@4.5bar & 0.14 bar $(3.0 \%)$ \\
\hline Pressure loss@1bar & 0.13 bar $(12.8 \%)$ \\
\hline Thrust@4.5bar & $4.4 \mathrm{mN}$ \\
\hline Thrust@1bar & $0.9 \mathrm{mN}$ \\
\hline
\end{tabular}

Table 2-V: The lower limits for the dimensions of the valve are calculated. A deflection of 17.5 $\mathrm{mm}$ is assumed and the difference between the inner and outer diameter of the valve seat is assumed to be 600 um.

\subsubsection{Particle Filter}

The main purpose of the filter is to block particles which can get stuck inside the valve causing leakage. A perforated silicon membrane is designed to function as particle filter [14]. It is a robust membrane which is easily integrated in the modular design of the thruster and feeding which is presented in chapter 3. The main constraint for the filter is again the area of passage which should be much larger than the nozzle throat. With a porosity $p$ of $10 \%$ and the area of passage through 
the filter at least 100 (N) times larger than the nozzle throat the minimum area of the filter can be calculated:

$$
A_{f}=N \cdot A_{t} \cdot\left(\frac{100 \%}{p}\right)
$$

Table 2-VI shows the calculated filter area for the upper and lower limits. A 10\% filter porosity can be obtained by an hexagonal $2 \mu \mathrm{m}$ hole pattern with a $4 \mu \mathrm{m}$ spacing between the holes. Particles smaller than $2 \mu \mathrm{m}$ will be able pass through the filter and still can cause leakage in the valve. It is possible to make a filter with nano-sized pores to reduce the leakage induced by small particles [15]. Regardless of the size of the sieve, the valve has to be designed in such a way that it can cope with small particles anyway.

\begin{tabular}{|l|l|l|}
\hline & Upper limit & Lower limit \\
\hline Throat diameter $\left(d_{t b r o a t}\right)$ & $131 \mu \mathrm{m}$ & $90 \mu \mathrm{m}$ \\
\hline Throat area $\left(A_{t}\right)$ & $1.35 \cdot 10^{-8} \mathrm{~m}^{3}$ & $6.4 \cdot 10^{-9} \mathrm{~m}^{3}$ \\
\hline Minimum filter size & $1.35 \cdot 10^{-5} \mathrm{~m}^{3}$ & $6.4 \cdot 10^{-6} \mathrm{~m}^{3}$ \\
\hline
\end{tabular}

Table 2-VI: The upper and lower limits for the dimensions of the filter are calculated. The porosity of the filter is assumed to be $10 \%$.

\subsection{Conclusion}

The functional design of a cold gas micro propulsion system is presented. It consists of the propellant storage and the thruster and feeding system. For the components of the latter - i.e. the filter, valve and nozzle - the characteristic dimensions are calculated. It is shown that these dimensions are indeed in the micrometer range and thus MST is a suitable technology to use.

\subsection{References}

[1] Le Mair A.F., Cold gas micro propulsion - a technology development -, TNO Defence, Security and Safety, Rijswijk (2006).

[2] O. B. George P. Sutton, Rocket Propulsion Elements: an introduction to the engineering of rockets, 7th edition, John Wiley \& Sons, inc., New York (2001).

[3] http://cubesat.atl.calpoly.edu/. 
[4] Moerel J.-L., Hogedoorn A. and Dekker E., System Requirements Document, MicroPropulsion System for MiSAT, TNO Defence, Security and Safety, Rijswijk (2006).

[5] Rackemann N. J., Sanders H. M. and van Vliet L. D., Design and development of a propulsion system for a cubesat - Based on solid propellant cool gas generator technology -, ALA A 57th International Astronautical Congress, $L A C$ vol. 5 (2006), pp. 3434-3442.

[6] Sanders H.M., Boscher J.M., Hogedoorn A.T., Moerel J.L.P.A., Zandbergen B.T.C. and Louwerse M.C., System analysis and development of a cool gas generator based micropropulsion system, ESA MNT 6 Round table 6 on micro/nano technologies for space, Noordwijk, The Netherlands (2007).

[7] Larson W. J. and Wertz J. R., Space Mission Analyses and Design, 2nd edition, Microcosm inc. and Kluwer Academic Publishers, Dordrecht (1992).

[8] Fleischer R. L. and Price P. B., Charged Particle Tracks in Glass, Journal of applied physics 34 (1963), pp. 2903-2904.

[9] Munson B.R., Young D.F. and Okiishi T.H., Fundamentals of fluid mechanics, John Wiley \& Sons, Inc. (2002).

[10] Woias P., Micropumps--past, progress and future prospects, Sensors and Actuators B: Chemical 105 (2005), pp. 28-38.

[11] Fazal I., Development of a gas microvalve based on fine- and micromachining, Tranceducer Science and Technology, University of Twente, Enschede (2007).

[12] http://www.piezo.com/.

[13] Browne V.d'A. and John J.E.A., Vacuum radial flow from the viscous through the free molecule regime, V acuum 20 (1970), pp. 525-533.

[14] Unnikrishnan S., Jansen H.V., Berenschot J.W. and Elwenspoek M.C., Wafer scale nano-membranes supported on a silicon microsieve using thin-film transfer technology, Journal of Micromechanics and Microengineering 18 (2008), p. 064005.

[15] Tong H. D., Jansen H. V., Gadgil V. J., et al., Silicon Nitride Nanosieve Membrane, Nano letters 4 (2004), pp. 283-287. 


\section{3}

\section{Modular system design}

Our vision on the development of a complex system consisting of several functional parts is presented. Miniaturization, integration and packaging play a crucial role in developing a modular platform for complex systems. A simple baseline package is chosen first. By adjusting the MST devices to fit the package integration is simplified. This is a new approach to put several, independently tested, functional MST devices together to perform a complex task. 


\subsection{Introduction}

The feeding and thruster system consists of several functional parts; a filter, a valve, a nozzle, and electronics. The electronics controls the actuation of the valve and handles the read out of the pressure- and temperature sensor. These parts need to be connected mechanically and electrically and furthermore a fluidic connection is required for them to be able to function together. In the macro-world fluidic devices are often connected by piping. The devices and pipes are big and can easily be connected by human hands. When things are miniaturized this becomes a more difficult task. In this chapter a modular platform is presented to connect the functional parts, which we will call functional modules.

Paragraph 3.2 discusses the design approach for systems based on MST that consist of multiple functional modules. Then in paragraph 3.3 the modular feeding and thruster system is presented. This chapter closes with some concluding remarks in paragraph 3.4 .

\subsection{Design approach}

Section 3.2.1 shortly discusses two mainstream approaches for building complex MST systems that are found in literature. Our vision on a modular design approach is stressed in section 3.2.2.

\subsubsection{Literature}

A couple of research groups are working on the miniaturization of micro propulsion systems by means of MST. In chapter 1 several of such propulsion systems passed the revue. We focus on the systems that use gas as propellant. Such a system is build from several functional parts which together perform a complex task. Two approaches are found in literature to integrate - say: put together - these functional parts with MST.

The first approach for building complex fluidic systems is based on the following techniques. Bulk micro-machining is used to make 2D extruded shapes in silicon which is characteristic for MST. Then several 2D structured layers are stacked to obtain a functional device. Probably one of the most complicated examples of this is the micro-engine developed by MIT [1]. They build a micro-engine of six stacked 
silicon wafers which includes a diffuser vane, compressor rotor, turbine rotor, journal bearing, thrust bearing, combustor and a nozzle guide vane. A schematic of this device is shown in Figure 3-1.

The micro-engine is a very complex system from technological point of view. The micro-engine consists of several functional parts that are fabricated in one and the same run. This has a significant drawback; when one part of the system has become defective during fabrication the whole device is useless. Finally, the packaging of such a system is quite complicated.

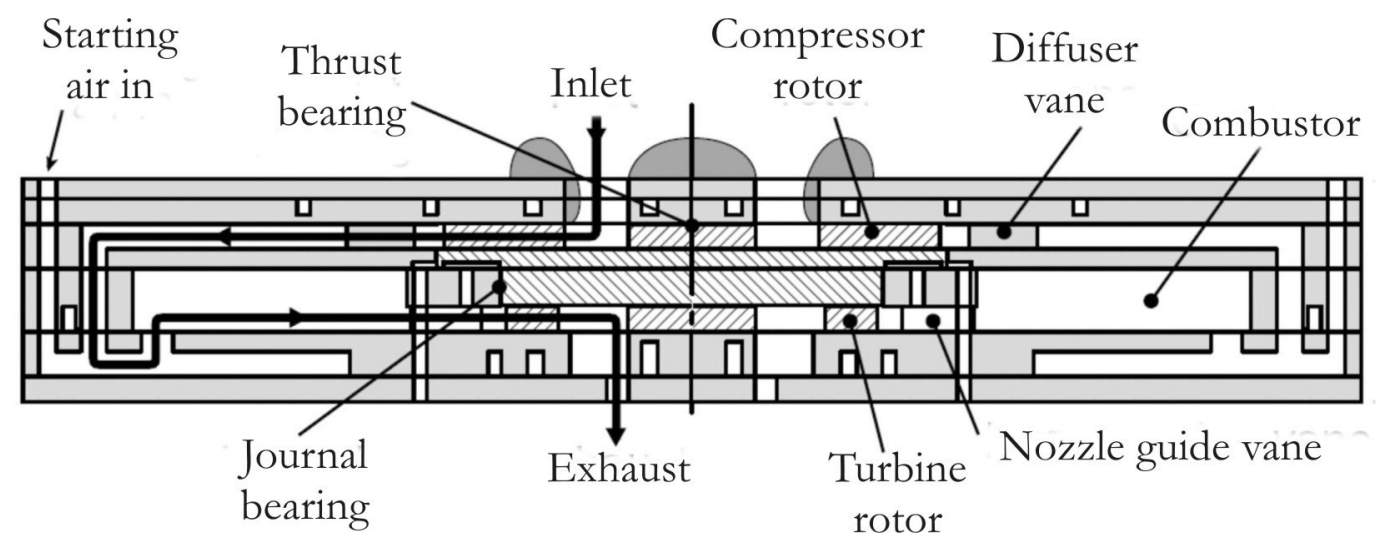

Figure 3-1: MIT micro-engine: $H_{2}$ demo engine with conduction-cooled turbine constructed from six silicon wafers. [1]

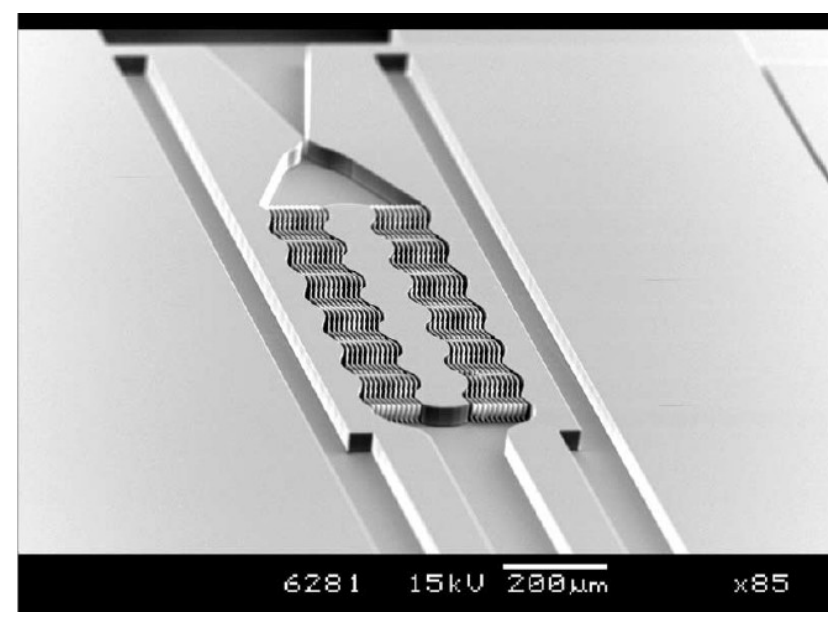

a)

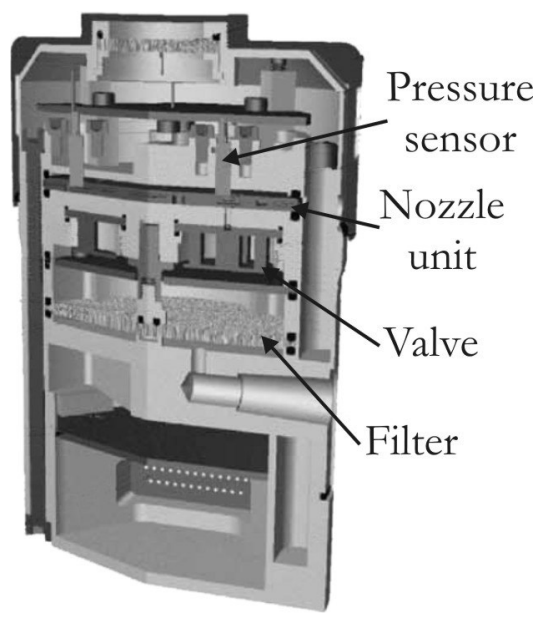

b)

Figure 3-2: Köbler micro-thruster [2] a) Nozzle unit. b) Hybrid cold gas micro-thruster system.

For the second approach all functional parts are manufactured separately in one or two silicon wafers. An example of this is the hybrid cold gas micro-thruster 
presented by Köhler et al [2]. It includes a nozzle unit with in front of it an integrated heat exchanger as shown in Figure 3-2.a. On the outside surface of the heat exchanger platinum thin film heater elements and temperature sensors are included. Besides the nozzle unit, this system includes two other MST manufactured devices, namely a filter and a valve. Figure 3-2.b shows the total cold gas micro-thruster system. Several functional parts are put together in a specifically designed package.

\subsubsection{Modular approach}

In the future, ultimate integration might be achieved by implementing all functionality in a single process run. However, at this moment most MST devices require a dedicated fabrication process, optimized for the task at hand. Integration of multiple functionalities means that the fabrication processes cannot be optimized for every dedicated function. Thus, concessions have to be made on the requirements of a single functionality. In the future it is envisioned that MST will be so mature that the design is less limited by the technology and further miniaturization will be possible by a higher degree of integration.

With the above in mind and acknowledging the fact that integration is limited by the technology, we prefer the manufacturing of functional devices as separate modules. This is pretty much the same approach as for the hybrid cold gas microthruster system of Köhler but pushed further. The fabrication process can then be optimized to the needs of every single module. This results in a more simplified fabrication scheme than when full integration is pursued, and thus a higher yield. Instead of a dedicated package we see the need for a simple, modular platform to put these functional parts together. It is often the packaging that makes MST devices still quite large and time consuming. Thus, in our vision on miniaturization, modularity and packaging are of utmost importance. By selecting a convenient package first and adjusting the MST part to fit the package, overall size and mass are reduced and modularity is obtained [3,4]. All functions are fabricated separately and a properly working unit is selected first, before integration. Depending on the requirements of the system, functionality can be added in a plug\&play fashion. When damaged during operation, some of these devices can even be replaced which is useful for terrestrial applications of this technology. 


\subsection{Modular feeding and thruster system}

In this paragraph the modular design of the feeding and thruster system is presented. In section 3.3.1 the baseline package is presented. Section 3.3.2 describes the electronics module and the filter module.

\subsubsection{Baseline glass tube package}

In this section it is described how a simple convenient package forms the baseline for a modular platform for several independent functional modules. The manufacturing procedure of this baseline package is discussed. Finally some characteristics of the package are considered like: the hermetic sealing properties, burst pressure and environmental issues.

The baseline package of modular platform is a glass tube bonded on silicon. Figure 3-3 shows the glass tube with a silicon component underneath it. This silicon component can contain any functionality according to the requirements of the system. In our case it is the valve module which is described in chapter 5. The glass tube is a convenient package because it is easily attached to silicon or other glass like materials by fusion bonding. It is functioning as a hermetically sealed package, fluidic interconnect as well as a macro support for the fragile MST components [4].

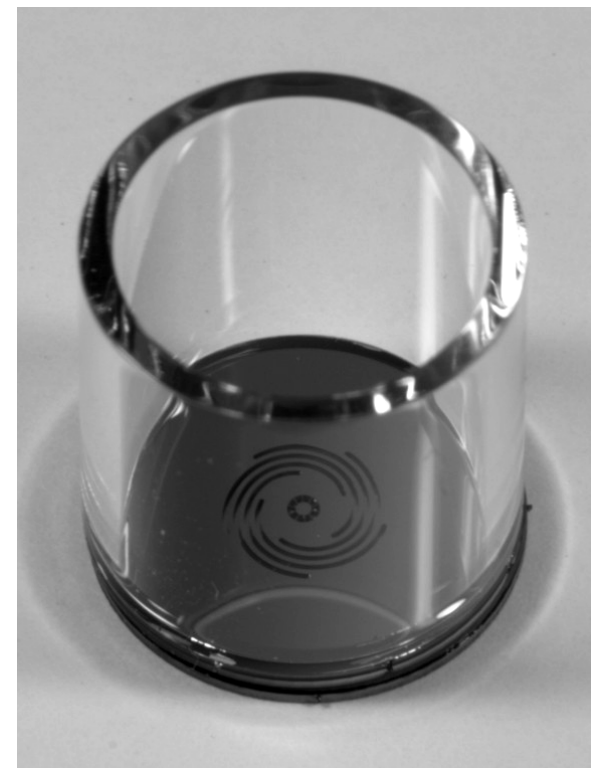

Figure 3-3: Baseline glass tube package 
The glass tube is fixed on the MST part by fusion bonding at elevated temperature [5]. First the glass tubes are diced to the right length and the bonding surface is polished [3]. Just before fusion bonding the glass tube is ultrasonically cleaned in ethanol. The glass tube is positioned on top of the clean silicon valve. The heat treatment is done in a Nabertherm LH 15/12 furnace in an air environment. The unit is heated up to $790^{\circ} \mathrm{C}$, maintained at this temperature for 30 minutes, after which it is gradually cooled down resulting in a hermetic seal between the glass tube and the silicon [6].

Figure 3-4 shows a schematic of the thruster and feeding system. It shows a glass tube bonded on a silicon-glass-silicon stack. This stack contains the valve structure and the conical converging-diverging nozzle. The valve and nozzle can be tested for functionality before the nozzle is attached to the valve by anodic bonding. The nozzle can also be characterized for thrust levels and thrust angle as stand alone. The valve requires the glass-silicon stack underneath for rigidity to avoid bending of the valve under tank pressure which causes leakage.

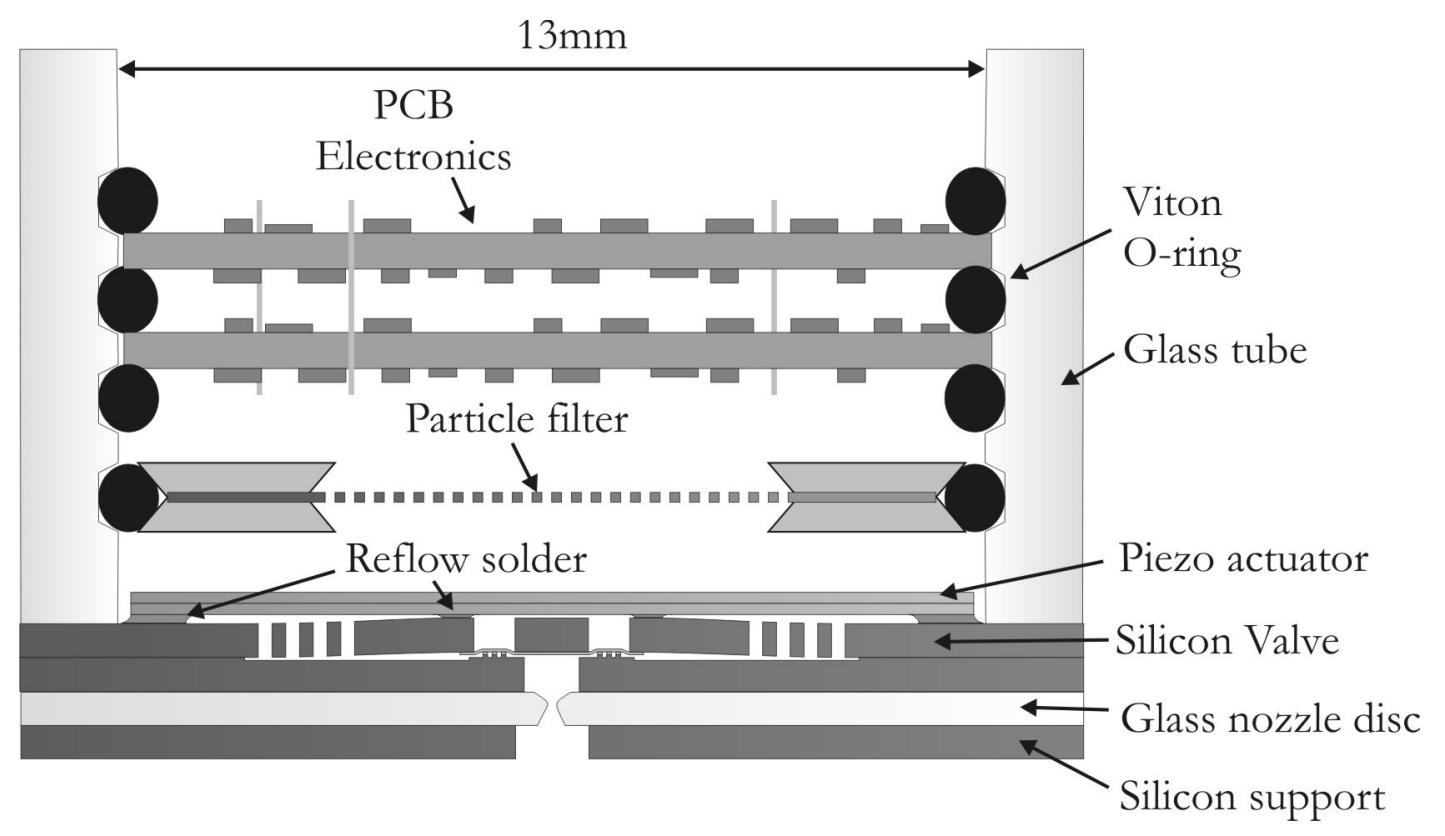

Figure 3-4: Schematic of the feeding and thruster system

The active valve is normally closed and can be opened by the piezo-disc actuator which is attached to the valve by reflow soldering. A piezo-disc with a diameter of $12.7 \mathrm{~mm}$ - performing with a stroke of $19.1 \mu \mathrm{m}$ - is used. The size of the piezo-disc actuator determines the diameter of the glass tube which is $13 \mathrm{~mm}$ inner- and $16 \mathrm{~mm}$ 
outer diameter [7]. When smaller thrust levels are required for more precise positioning of the satellite the throat of the nozzle will be smaller which allows a smaller area of passage through the valve. A smaller, commercially available, piezodisc actuator can then be used which measures $6.4 \mathrm{~mm}$ in diameter and has a specified deflection of $4.7 \mu \mathrm{m}$ [7]. The size of the system is mainly defined by the size of the actuator.

Several other functional modules can be integrated inside the tube by a simple technique. Powder blasted glass discs have a tapered sidewall. When two glass discs are bonded together a V-shape is obtained, as can be seen in Figure 3-5. A Viton O-ring fits around the bonded glass discs. In the inside of the glass tube a small groove can be made by precision machining techniques. When the glass stack is pushed in the tube the O-ring gets stuck in the groove. In this way the particle filter can be integrated. The electronics can also be integrated in a similar way. The circular printed circuit boards (PCB) that contain the electronics for the valve actuation and sensors can be suspended in between two O-rings as can be seen in Figure 3-4. These double sided PCB's contain electronics on both sides and the open vias function as electrical connection as well as passage for the gas flow towards the valve. One can imagine all kinds of modular systems stacked inside a glass tube, i.e. complex filtration systems or chemical reactors.

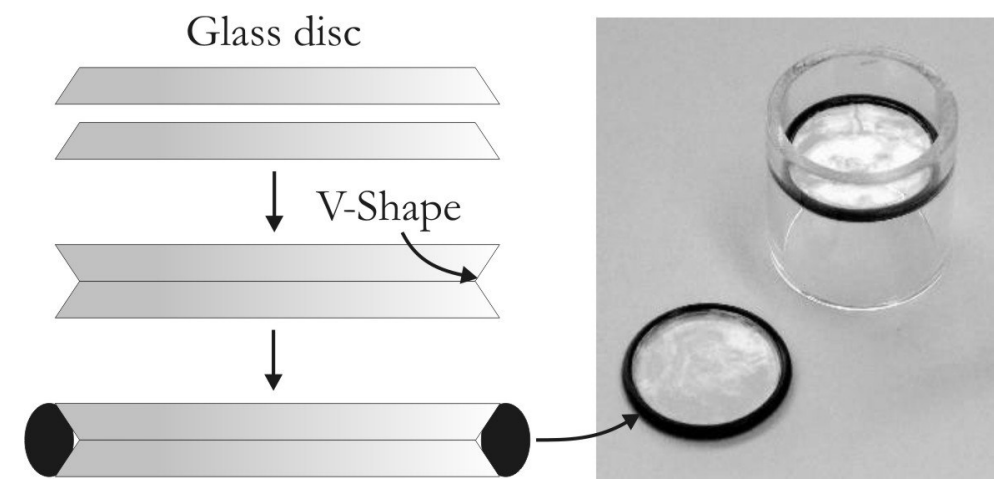

Figure 3-5: Functional system inside a glass tube 
The following characteristics of the baseline package are considered: hermetic sealing, burst pressure and some environmental aspects.

\section{- Hermetic sealing}

Here we only look into the leakage of the baseline package at room temperature. The leakage through the valve is discussed in Chapter 5. Two mechanism of leakage are considered for the presented system; leakage due to gas diffusion through materials and leakage through interfaces. Regarding the latter type, the bond between the glass and silicon is of interest. This bond has been tested and no substantial leakage could be measured [3]. For gas diffusion, the glass tube is considered. The diffusion of gas through silicon is virtually zero compared to the diffusion of gas through the glass tube $[3,8]$. The saturated diffusion rate, through the glass, is calculated by:

$$
\varphi=\frac{2 \pi \cdot L \cdot D \cdot S \cdot p}{\ln \left(r_{o} / r_{i}\right)}
$$

The glass tube has a height $(\mathrm{L})$ of $8 \mathrm{~mm}$ and an inner $\left(\mathrm{r}_{\mathrm{i}}\right)$ and outer $\left(\mathrm{r}_{\mathrm{o}}\right)$ radius of $6,5 \mathrm{~mm}$ and $8 \mathrm{~mm}$ respectively. The diffusion coefficient (D) of Helium through the glass is $54 \cdot 10^{-8} \mathrm{~cm}^{2} / \mathrm{min}$ and the Solubility (S) is $0.8 \cdot 10^{-2}$. For Helium at a pressure of $4.5 \mathrm{bar}$ the calculated leakage through the glass tube is $4.7 \cdot 10^{-6} \mathrm{sccm}$. This leakage is 36 times less than the allowed leakage and for nitrogen it will be even 3 decades lower [9].

\section{- Pressure testing}

The system has to be able to withstand a pressure of 10 bar. When the system bursts under a high pressure load, small pieces of silicon and glass are scattered. Propulsion is not possible anymore and even worse, the fragments of silicon and glass can damage other parts of the satellite. Therefore the glass tube package is tested for burst pressure.

Two tests have been performed. For the first test a glass tube is bonded to a $525 \mu \mathrm{m}$ thick silicon disc. The device is placed in a specifically designed sample holder, which is shown in Appendix C. While carefully increasing the pressure on the system, the pressure is monitored. At a pressure of $4.5 \mathrm{bar}$ the system bursts. Figure 3-6 shows the broken glass tube package. One can see there is still silicon left on the glass. The silicon disc can be considered as a membrane which bends under the 
applied pressure. When the stress becomes too high a crack is initiated. This always occurs in the glass since this is the weaker material. Under certain conditions this crack can propagate into the silicon which is described by Mogulkoc [6]. The result of such a rupture is shown in Figure 3-6.a. The crack can also propagate through the glass tube which results in the broken tube as shown in Figure 3-6.b.

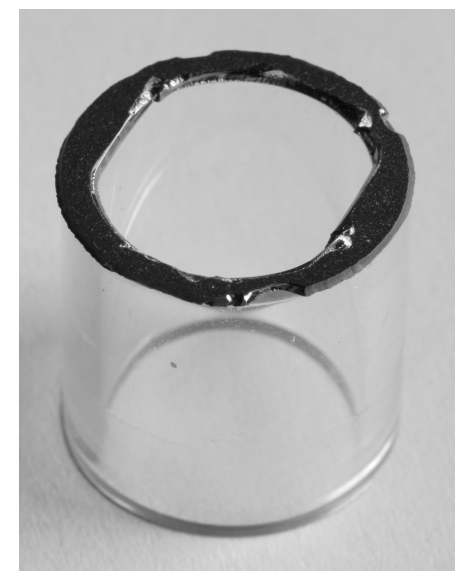

a)

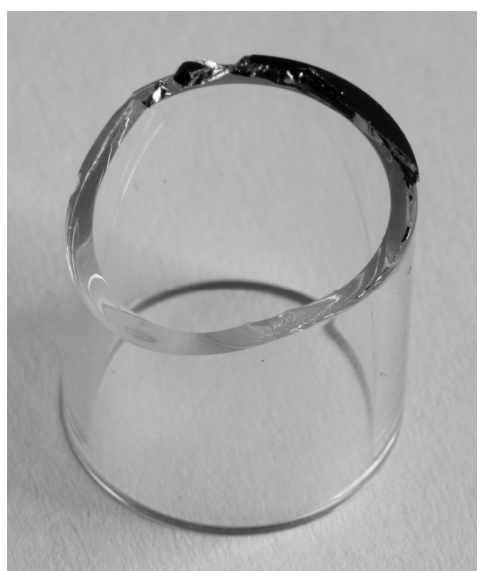

b)

Figure 3-6: Glass tube package after burst pressure test. (inner and outer diameter are $13 \mathrm{~mm}$ and $16 \mathrm{~mm}$ )

For constant pressure, when increasing the thickness of the silicon disc the stress is distributed more equally reducing the peak stress on the inner radius of the tube near the bond interface. Therefore, an increase of the thickness results in a higher burst pressure.

For the second test the glass tube is bonded to the silicon valve supported by a glass and silicon disc as shown in Figure 3-4. The only difference is that the silicon disc underneath is without an exit hole. The valve, glass disc and silicon disc have a thickness of $760 \mu \mathrm{m}, 500 \mu \mathrm{m}$ and $525 \mu \mathrm{m}$, respectively. This adds up to a total thickness of $1785 \mu \mathrm{m}$ for the entire stack. The system is tested up to 12 bar without any rupture. More information about the bond strength between the glass tube and silicon can be found in [6].

\section{- Environmental aspects}

The glass tube is bonded to the silicon at a temperature of $790^{\circ} \mathrm{C}$ and then cooled down to room temperature. In the specified temperature range of $-40^{\circ} \mathrm{C}$ to $60^{\circ} \mathrm{C}$ the thermal expansion coefficient between glass and silicon is quite close. Therefore 
very little internal stress is induced between the glass and silicon and thus no problems are expected.

The glass tube package has to be connected to the Titanium gas storage tank. The thermal expansion coefficient between glass and Titanium alloy $\mathrm{TiAl}_{6} \mathrm{~V}_{4}$ does differ considerably. Direct contact between these materials causes stress in the glass tube package and it might break. Therefore, the glass tube package is suspended by orings to avoid direct contact and to introduce an elastic buffer. These o-rings fix the glass tube package in place and act as dampers for the extreme vibrations and shocks during the launch. This is important since glass and silicon are brittle materials and might break when handled to roughly. Vibrations are random over a frequency range of 20 to $2000 \mathrm{~Hz}$. Furthermore, shockwaves induce a mechanical response over a wide frequency range which is specified as the peak acceleration. Data on the vibrations and shocks for various launch vehicles can be found in [10]. At last radiation is considered. This radiation can be particles traveling with speeds close to the speed of light; i.e. cosmic rays, or highly energetic electromagnetic waves; extreme ultra violet. The part that is most sensitive for radiation is the electronic module. The electronics is positioned inside the glass tube. The propulsion system, including the glass tube packaged feeding and thruster system, is positioned inside the protective body of the satellite. Before being able to damage the electronics, high-energy particles first have to penetrate the protective satellite body and the glass tube. In glass, trails of damage are found due to heavily ionizing particles that pass through the material [11]. Their tracks have a length in the order of 10 to $20 \mu \mathrm{m}$. With a thickness of $1.5 \mathrm{~mm}$, the glass tube is a good protective body for the electronic module.

\subsubsection{Functional modules}

In this paragraph the electronic piezo-driver module for valve actuation is presented first followed by the mechanical filter module.

\section{- Piezo driver module}

Since there is a power supply of typically $5 \mathrm{~V}$ onboard of a micro-satellite, additional electronics is needed to obtain the $180 \mathrm{~V}$ that is required to actuate the piezo-disc. Power consumption is of utmost importance in space applicable devices, and thus requires a design which is as energy thrifty as possible. A peak power of $1 \mathrm{~W}$ may be 
used at maximum and on average a power of less than $100 \mathrm{~mW}$ is preferred. Another important requirement is the size and mass of the electronics which should reduced as much as possible. These two requirements, energy thrifty on the one hand and small size on the other, are complementary and a fair trade off has to be made.

To obtain an accurate impulse bit the piezo-disc has to go from zero deflection to a full deflection of $19.1 \mu \mathrm{m}$ in $1 \mathrm{~ms}$ and vice versa. The piezo-disc reaches full deflection when a stable $180 \mathrm{~V}$ is reached. The capacity of the $12.7 \mathrm{~mm}$ diameter piezo actuator is $4.3 \mathrm{nF}$ and the energy to be stored in this piezo element is then calculated by: $1 / 2 \mathrm{CV}^{2}=70 \mu \mathrm{J}$. This energy should be delivered to the piezo-disc within $1 \mathrm{~ms}$. To be able to do this, a buffer capacitor, with a chosen capacity approximately 16 times larger than the capacity of the piezo-disc, is used as an energy reservoir. This capacitor is charged up to $190 \mathrm{~V}$ to store $1,2 \mathrm{~mJ}$ of energy. By switching the piezo-disc in parallel with the buffer capacity, the energy is distributed over the two capacitors and the total voltage drops to $180 \mathrm{~V}$. The speed at which this takes place is determined in the electronic design by limiting the current and this can be done well within $1 \mathrm{~ms}$.

When the repetition rate of actuation is very low we can choose to charge the buffer capacitor within a longer time. A charge- or wakeup time of $100 \mathrm{~ms}$ would require a theoretical average power of $30 \mathrm{~mW}$. Another possibility is to keep the buffer capacitor charged at all times and adjust for leak-currents, meaning the thruster is always ready to be actuated.

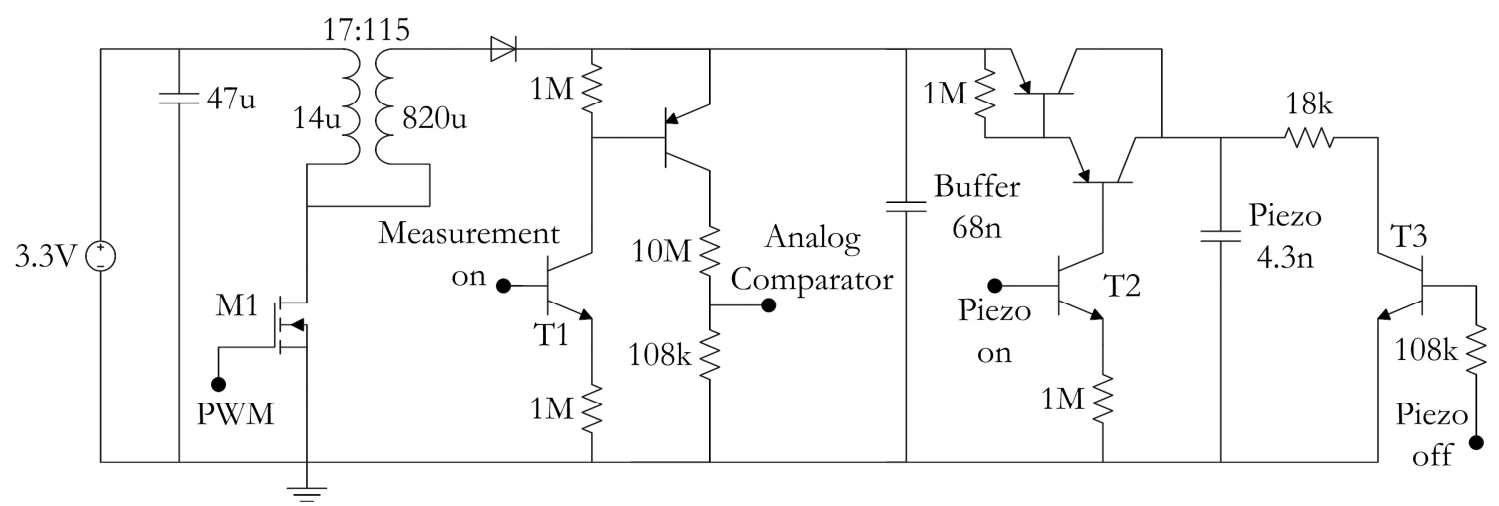

Figure 3-7: Electronic circuit of the piezo driver. For the complete circuit see Appendix A 
The buffer capacitor is charged by the circuit shown in Figure 3-7, which is based on the flyback step-up principle. When the buffer capacitor is completely charged the piezo-disc can be actuated. During actuation the voltage is measured to be able to maintain the desired voltage. Measurement of the voltage requires energy and therefore is done only once in a while instead of continuously.

A micro controller - not shown, but presented in Appendix A - is used to control the piezo actuator circuit shown in Figure 3-7. The controller is a low power 8-bit microcontroller of Atmel, the ATtiny24. This microcontroller is available in a very small package measuring $4 \times 4 \mathrm{~mm}$ and a height of $0.75 \mathrm{~mm}$. It can generate a pulse width modulated (PWM) signal for charging the buffer capacity which is applied to the MOSFET indicated by M1. The measurement circuit is activated by transistor $T 1$ and the buffer voltage can then be measured with an analog comparator input of the microcontroller. While the gate of $T 3$ is driven low and $T 2$ is driven high the piezo-disc is actuated. The piezo element is discharged when T2 is driven low and T3 driven high. This microcontroller has the possibility to measure its temperature which gives an indication of the temperature of the piezo-disc and nitrogen gas. This is an important parameter to predict the thrust.

The circuit works at voltages between a 3.3 and 5VDC and communication is possible with a common two-wire protocol. Commands can be given to actuate the piezo-disc for a certain amount of time according to the desired impulse that is required for a correction in position. Figure 3-8 gives an event timeline of a typical thrust cycle and shows the corresponding required power during each stage. The power consumption is measured in each state separately and reflected in Figure 3-8. In the idle state the microcontroller is in power-down mode and the total power that is used is less than $1 \mathrm{~mW}$. The most power is required during wake up in which the buffer capacitor is charged, i.e. $45 \mathrm{~mW}$ for $100 \mathrm{~ms}$. This can be reduced considerably if a longer charging time is chosen. When the buffer capacitor is charged, power is required to compensate for leak currents and to keep it charged at $190 \mathrm{~V}$. This requires a power of $25 \mathrm{~mW}$. A power of $40 \mathrm{~mW}$ is required during actuation to maintain the $180 \mathrm{~V}$. In appendix A a component list and the microcontroller program can be found. Also some measurements are included of the voltage over the piezo-disc. It is shown that from the moment the actuation starts it takes approximately $400 \mu$ s to reach a stable voltage. The piezo element is discharged within $700 \mu \mathrm{s}$. Both values fulfill the $1 \mathrm{~ms}$ requirement. The ripple on the 
$180 \mathrm{~V}$ has been measured during actuation. The amplitude of the ripple is $0.5 \mathrm{~V}$. After the thrust cycle the system returns to the idle state. The electronic components fit on a circular double sided printed circuit board with a diameter of $12 \mathrm{~mm}$. This is small enough to fit inside the glass tube above the valve. Figure 3-9 shows the electronic module.

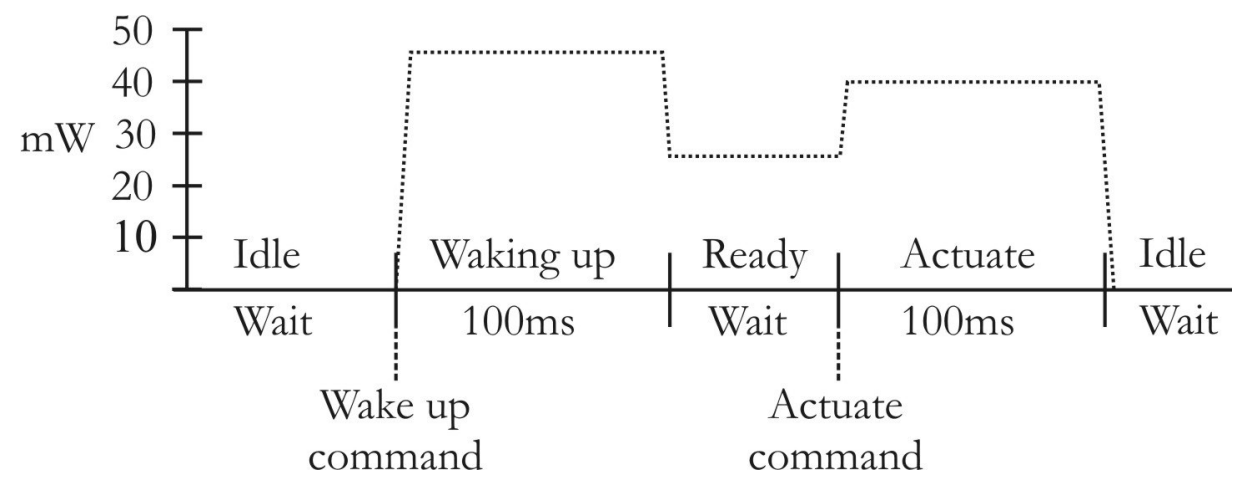

Figure 3-8: Event timeline

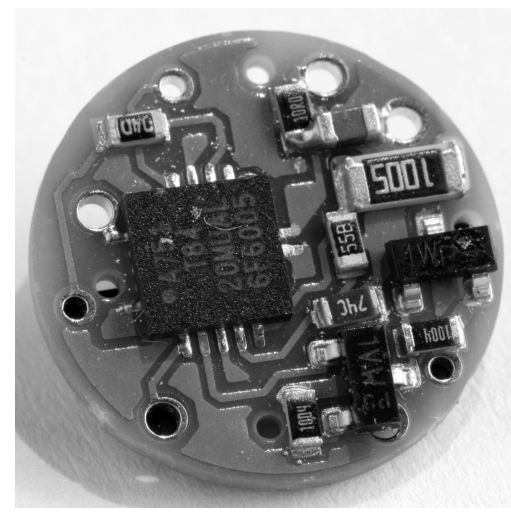

a)

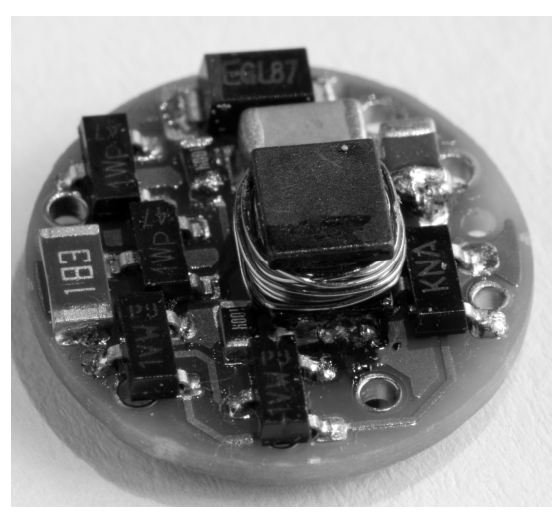

b)

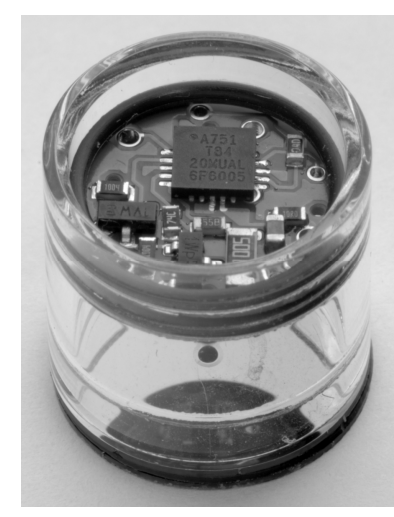

$c$

Figure 3-9: Piezo driver module a) frontside b) backside c) electronics suspended inside the glass tube

\section{- Filter module}

A filter module is fabricated which inhibits large particles getting stuck in the valve. A $100 \mu \mathrm{m}$ thick silicon membrane perforated with $5 \mu \mathrm{m}$ holes is fabricated by Unnikrishnan [12]. If needed, the perforated silicon membrane can be used as rigid support for nano-sized filters [13]. Two glass rings are powder blasted resulting in the typical inclined sidewall as is shown in Figure 3-5. The glass rings are attached 
to both sides of the silicon filter by anodic bonding. Figure 3-10 shows the filter inside the glass tube package suspended by o-rings.

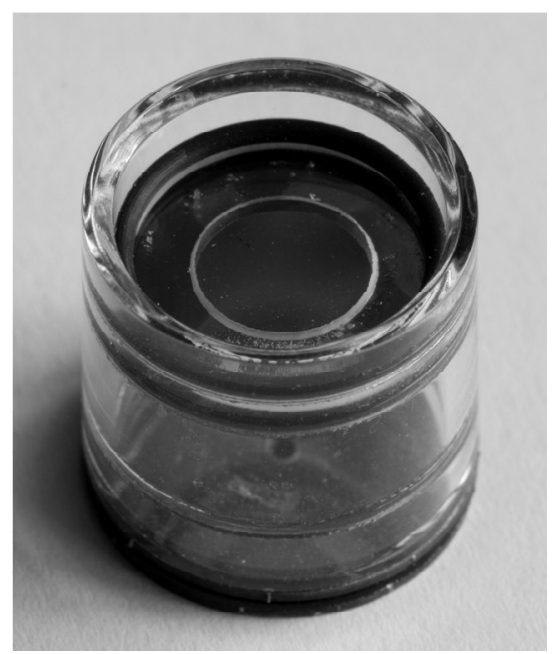

Figure 3-10: Filter module inside the glass tube package

\subsection{Conclusion}

In this chapter a baseline package is presented which forms the basis of a modular platform for MST devices. A simple glass tube is used as hermetically sealed package and tested up to $12 \mathrm{bar}$. It is functioning as a fluidic interconnection and a macro support for the functional modules. The valve and nozzle module are fusion bonded on top of the glass tube package. Other functional modules - like the electronics and filter module - are implemented by suspending them inside the glass tube by o-rings. In this way functional modules are put together which form the feeding and thruster system.

\subsection{References}

[1] Epstein A.H., Millimeter-Scale, Micro-Electro-Mechanical Systems Gas Turbine Engines, J. Eng. Gas Turbines Power 126 (2004), pp. 205-222.

[2] Köhler J., Bejhed J., Kratz H., et al., A hybrid cold gas microthruster system for spacecraft, Sensors and Actuators A: Physical 97-98 (2002), pp. 587-598. 
[3] Mogulkoc B., Jansen H. V., ter Brake H. J. M. and M. C. Elwenspoek, Borosilicate Glass (DURAN®) Tubes as Micro-Fluidic Interconnects, Proceedings of the 19th micromechanics Europe conference, Aachen, Germany (2008), pp. 283-286.

[4] Unnikrishnan S., Jansen H. V., Berenschot J. W., et al., A method for making a glass supported system, such glass supported system, and the use of a glass support therefore In: E. P. Office, Editor, Application No./Patent No. 08007746.4-2111 (2008).

[5] Fazal I., Berenschot J.W., de Boer R., Jansen H. V. and Elwenspoek M. C., Bond strength tests between silicon wafers and Duran tubes, Transducers (2005), pp. 936-939.

[6] Mogulkoc B., Jansen H.V., Berenschot J.W, ter Brake H.J.M., Knowles K.M. and Elwenspoek M.C., Characterization of MEMS-on-tube assembly: reflow bonding of borosilicate glass (Duran ${ }^{\circledR}$ ) tubes to silicon substrates, J. Micromech. Microeng. 19 (2009) 085027.

[7] http://www.piezo.com/prodbm5disk.html.

[8] Rogers W. A., Buritz R. S. and Alpert D., Diffusion Coefficient, Solubility, and Permeability for Helium in Glass, J. Appl. Phys. 25 (1954), p. 868.

[9] Roth A., Vacuum Technology, 2nd revised edition, Elsevier Noth Holland Publishing Company (1982).

[10] Larson W. J. and Wertz J. R., Space Mission Analyses and Design, 2nd edition, Microcosm inc. and Kluwer Academic Publishers, Dordrecht (1992).

[11] Fleischer R. L. and Price P. B., Charged Particle Tracks in Glass, Journal of applied physics 34 (1963), pp. 2903-2904.

[12] Unnikrishnan S., Jansen H.V., Berenschot J.W. and Elwenspoek M.C., Wafer scale nano-membranes supported on a silicon microsieve using thin-film transfer technology, Journal of Micromechanics and Microengineering 18 (2008), p. 064005.

[13] Unnikrishnan S., Jansen H.V., Falke F.H., et al., Transition low through an ultra-thin nanosieve, Nanotechnology 20 (2009) 305304. 



\section{4}

\section{Nozzle fabrication ${ }^{*}$}

A nozzle is used to increase the velocity of the exhaust. By accelerating the gas the efficiency of the propulsion system is increased. The nozzles that have been made with MST have a 2D extruded shape. Three fabrication methods are explored to make a truly 3D conical converging-diverging nozzle shape.

* This chapter is based on: Louwerse M.C., Jansen H.V., Groenendijk M.N.W. and Elwenspoek M.C., Nozzle Fabrication for Micro Propulsion of a Micro Satellite, J. Micromech. Microeng. 19 (2009). 


\subsection{Introduction}

In a propulsion system, the nozzle is primarily used to increase the velocity of the exhaust. The higher the effective outlet velocity the more thrust is generated with the same amount of expelled mass and therefore efficiency is increased. For the formation flying of two micro satellites where the distance between the satellites is controlled a nozzle is required delivering a thrust in the order of 1 to $10 \mathrm{mN}$ [1]. The typical shape of a de Laval nozzle is shown in Figure 4-1. A subsonic flow enters the converging part of the nozzle and reaches Mach 1 in the throat of the nozzle. The gas is further accelerated in the diverging part and expelled through the nozzle exit at supersonic speed.

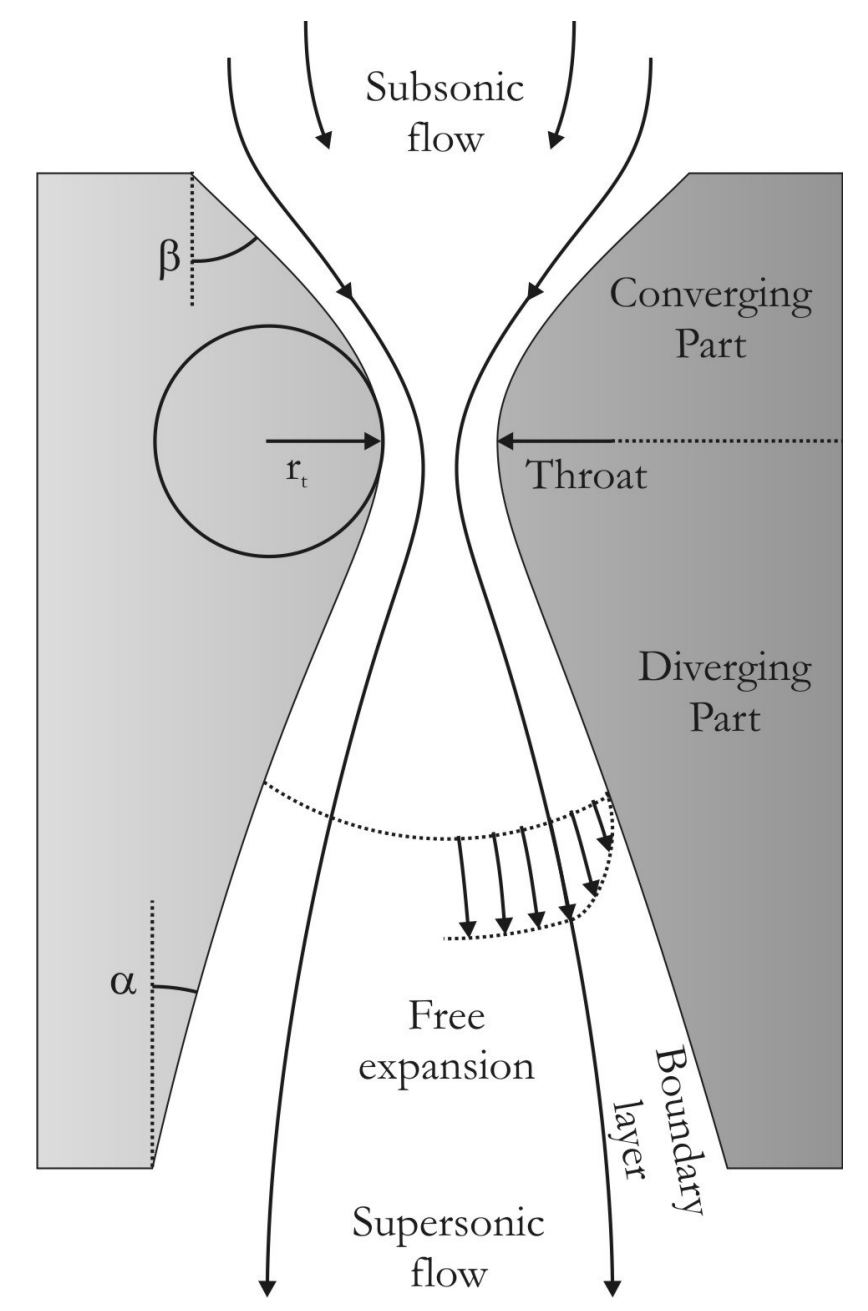

Figure 4-1: Typical 'de Laval' nozzle geometry 
Previously, nozzles have been fabricated by means of micro system technology. Two types of MST nozzles can be distinguished, the 2D extruded nozzle shape and the 3D conical nozzle shape. Bayt et al. made 2D extruded nozzles by means of deep reactive ion etching [2]. The nozzles fabricated by this etch technique are limited in their geometry to extruded shapes and therefore the efficiency of these nozzles is low compared to the 3D conical variant. In this paper we focus on the fabrication of a 3D conical converging-diverging nozzle.

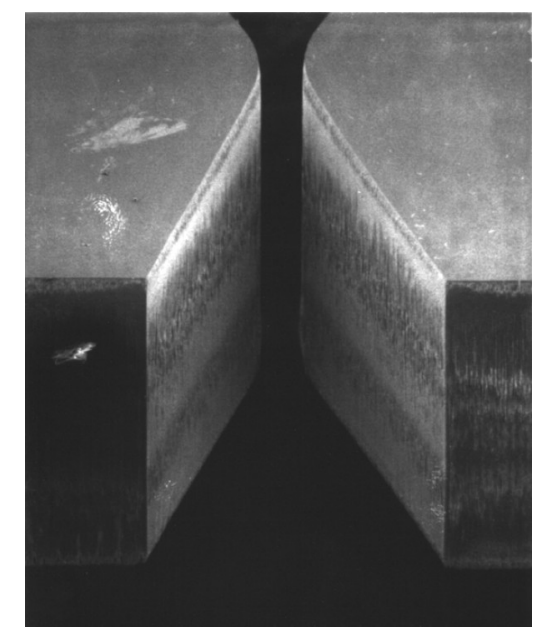

Figure 4-2: 2D extruded nozzle of Bayt. [2]

To enable integration with the other parts of the MST thruster and feeding system the nozzle should be made of silicon or glass. Important parameters for the nozzle are i) profile control, ii) surface roughness and iii) symmetry [3].

i) The most important aspects of the nozzle profile are the throat and exit diameter and the half angle (a) of the diverging bell shaped part of the nozzle. The throat area determines the mass flow through the nozzle and the ratio between the throat area and the exit area determines the outlet velocity and outlet pressure of the exhaust flow. To obtain the required thrust the throat diameter should be in between 50 and $300 \mu \mathrm{m}$, depending on the inlet pressure and expansion ratio.

The diverging part of the nozzle has a half angle (a) between $15^{\circ}$ and $20^{\circ}$. The angle of the converging part $(\beta)$ of the nozzle is typically $30^{\circ}$, but is less important for the performance. The radius of curvature $\left(r_{t}\right)$ of the throat of a 'de Laval' nozzle, is 
typically equal to the throat diameter. A nozzle throat with a sharp corner widens the fluid boundary layer reducing the generated thrust.

ii) Flow separation and shock formation in the nozzle degrade the performance of the nozzle significantly. To prevent this, the roughness of the sidewall should be minimal, especially in the throat. The sidewall roughness also increases the fluid boundary layer reducing performance.

iii) Another important issue is the symmetry of the nozzle with regard to the thrust vector. An attitude control system enables the satellite to align the micro-thruster with its velocity vector. Such a control system consists of sensors to determine the attitude of the satellite, i.e. magnetometer, sun sensor and/or gyroscope, and of actuators to change the attitude, i.e. magnetorquers and/or reaction wheels. To do formation flying only one thruster is enough which is positioned in such a way that the thrust vector is going through the centre of mass of the satellite. Misalignment of the thrust vector results in a disturbance torque exerted on the satellite which has to be corrected by the attitude control system. The positioning of the thrust vector of the nozzle with respect to the satellite's centre of mass should be done with high accuracy. To make this task of aligning as easy as possible, as it is complex enough, the thrust vector should be precisely at right angle of the nozzle exit plane.

Three potential technologies are explored to make a conical converging-diverging nozzle, all with their own typical characteristics. The first method is deep reactive ion etching (DRIE). By tuning the process recipe we tried to control the profile of an etched hole to obtain the nozzle shape. For the second method femtosecond laser machining (FLM) is examined. The energy in the laser spot is used to tune the angle of the nozzle sidewall. The last method describes the fabrication of a glass nozzle by means of powder blasting and a heat treatment. The powder blasting process results in a hole with the desired inclined angle for the diverging part of the nozzle. The heat treatment determines the radius of curvature of the throat. 


\subsection{Experiment}

In this paragraph the experimental procedure and the required equipment is discussed for the three fabrication methods.

i) The first method uses deep reactive ion etching (DRIE) to shape the nozzle in a $100 \mathrm{~mm}$ diameter and $525 \mu \mathrm{m}$ thick $<100>$ silicon wafer [4]. The wafers where spin coated with an Olin 907-17 photoresist layer and patterned with 50 $\mathrm{mm}$ diameter circular features. After lithography the wafers were pre-baked to avoid cracking of the photoresist layer under cryogenic temperature. The DRIE system used in this study is the Alcatel/Adixen AMS $100 \mathrm{SE}$ DRIE system. In all experiments the $\mathrm{SF}_{6}$ flow was set at $400 \mathrm{sccm}$, ICP $2500 \mathrm{~W}$ and temperature $-120^{\circ} \mathrm{C}$. The rest of the process parameters where tuned to obtain a negative taper. The cross-sections are obtained by breaking the silicon wafers along its crystalline structure.

ii) For the second method, which is femtosecond laser machining (FLM), experiments where done on $100 \mathrm{~mm}$ diameter and $525 \mu \mathrm{m}$ thick $<100>$ silicon wafers. For the experiments a commercial Coherent system comprising a Vitesse Duo oscillator/seed laser and $\operatorname{Reg} A$ amplifier is used that delivers pulses with durations of $200 \mathrm{fs}\left(\mathrm{fs}=10^{-15} \mathrm{~s}\right.$ ) and energies of $4 \mu \mathrm{J}$. The central wavelength of this laser is $800 \mathrm{~nm}$. The laser was set to a repetition rate of $50 \mathrm{kHz}$. A galvo scanner was used to manipulate the laser spot over the workpiece. The laser beam was focused by an $\mathrm{f}-$ theta lens with a focal distance of $100 \mathrm{~mm}$. This leads to a focused spot size of $25 \mu \mathrm{m}$. After treatment with the laser the wafers were ultrasonically cleaned to remove residual particles. Again, cross-sections are obtained by carefully breaking the silicon wafers.

iii) For the method which includes powder blasting and a heat treatment we used $100 \mathrm{~mm}$ Pyrex glass wafers. A $100 \mu \mathrm{m}$ thick foil (BF410) is used as mask material and patterned with circular features having a diameter of $200 \mu \mathrm{m}, 300 \mu \mathrm{m}$ and $400 \mu \mathrm{m}$. For the powder blasting process a beam of $29 \mu \mathrm{m} \mathrm{Al} \mathrm{A}_{2} \mathrm{O}_{3}$ particles is expelled from a nozzle and manipulated over the work-piece. The heat treatment is done in a Nabertherm LH 15/12 furnace in an air environment. The cross-sections are obtained by dicing with a diamond saw. 


\subsection{Fabrication methods}

\subsubsection{Deep Reactive ion etching}

The first fabrication method that is explored is deep reactive ion etching (DRIE), where plasma is used that consists of ionic and reactive (radical) species which are important in the etching of silicon [4]. The technique is used to make high aspect ratio features in silicon which is especially interesting for MST devices. An $\mathrm{SF}_{6}$ plasma is used from which the reactive species etch silicon in an isotropic manner. A gas is added which forms an etch inhibiting layer, also called passivation layer, on the etching surface. The inhibiting layer is selectively removed from the bottom of the etching surface by introducing a bias voltage, driving the ionic species directionally towards the wafer surface. Since the inhibiting layer is not removed from the sidewall, etching only occurs in the bottom of a feature and in this way directionality is obtained.

The basic idea for etching the nozzle is to combine an isotropic etch step for the converging part of the nozzle (Figure 4-3.a), with a negatively tapered etch step (Figure 4-3.b) having a diverging angle between $15^{\circ}$ and $20^{\circ}$. During both etch steps the same photo-resist mask is used which has $50 \mu \mathrm{m}$ circular features. When both etch steps are preformed consecutively it results in the shape as shown in Figure 4-3.c). An example of such a nozzle is shown in Figure 4-4. The depth of this nozzle shape is $260 \mu \mathrm{m}$ and the throat diameter is $76 \mu \mathrm{m}$. The angle of the diverging part of the nozzle is approximately $5^{\circ}$ which is not sufficient for a de Laval nozzle. As the critical part is the diverging angle this is studied next.

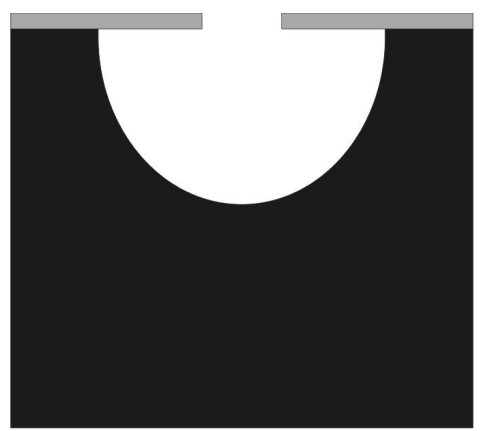

a)

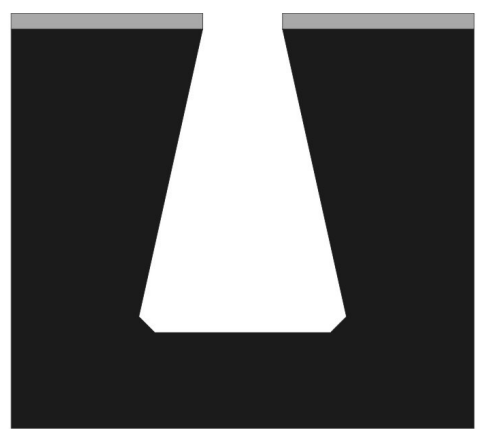

b)

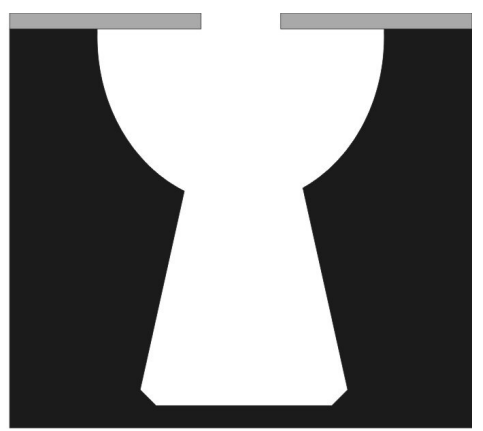

c)

Figure 4-3: a) Isotropic etch, b) directional etch, c) Combination of isotropic and directional etch 


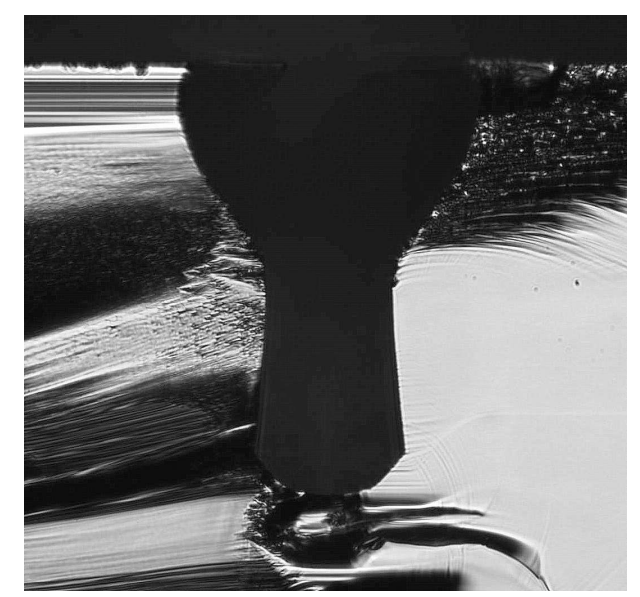

Figure 4-4: Resulting nozzle of a combination of isotropic and directional etching

The plasma glow shown in Figure 4-5 is a region where the plasma is highly conductive and rich of ions and radicals due to electronic impact. In between the plasma glow and the silicon a sheath is present where there is a depletion of electrons which prevents the plasma to exist and gives it insulating properties; i.e. the dark space. When a bias voltage is applied strong electrical fields develop in the sheath causing ions to accelerate from the plasma glow straight towards the etching surface.

The first effect that is explored to obtain a negative tapered profile is the ion angular distribution (IAD). Because of the collisions of ions with other species and their thermal motion in the plasma glow, ion dispersion will occur (Figure 4-5). At high pressure the ions encounter many collisions with gas molecules while travelling through the dark space. These collisions result in a flux of ions towards the silicon which is not perpendicular to the wafer surface. At a pressure of $10 \mathrm{~Pa}$, where the mean free path between collisions is $0.2 \mathrm{~mm}$ for $\mathrm{SF}_{6}$ at $300 \mathrm{~K}$, the ions which arrive at the silicon surface are distributed over an angle of $30^{\circ}$. The degree of collimation of the ion flux is expressed in the ion angular distribution (IAD) function [4]. When ions arrive under an angle with sufficient kinetic energy, they will cause etching of the sidewalls below the mask; i.e. a negative tapered profile which is a 'footprint' of the IAD. Figure 4-5.b shows the result of a high speed etch recipe based on the pulsed process optimized for a broad IAD and high kinetic energy, meaning high pressure and bias voltage [5]. The largest negative taper that was achieved with the Adixen tool was about $5^{\circ}$ and this is not enough for the diverging part of the nozzle. 


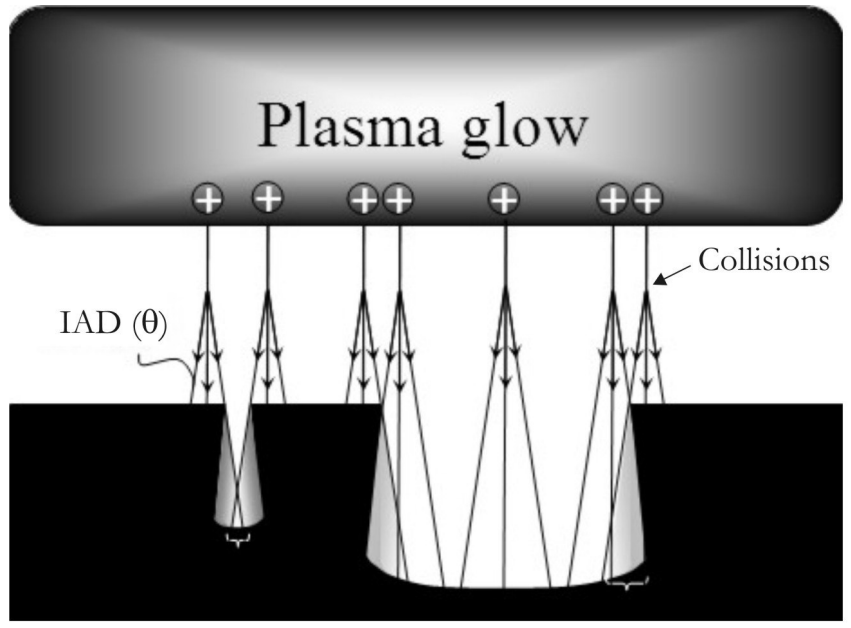

a)

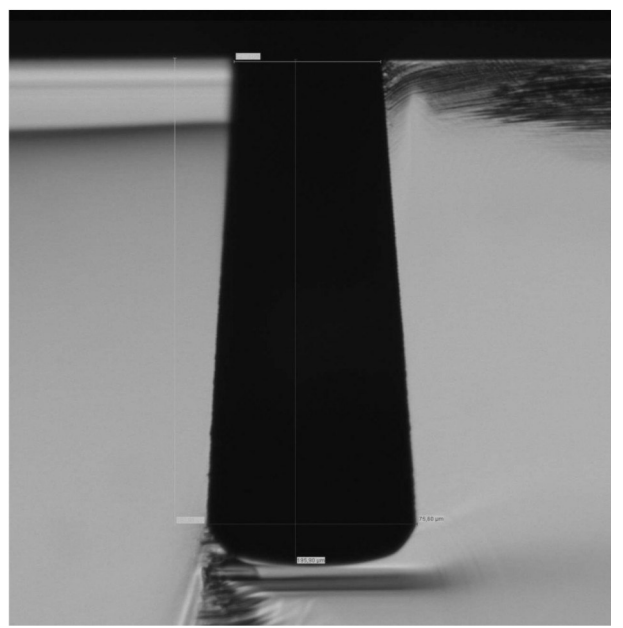

b)

Figure 4-5: a) LAD effect: ion angular distribution caused by collision in the dark space. b) Pulsed process settings: $\mathrm{SF}_{6} / \mathrm{CHF}_{3}: 400 \mathrm{sccm} / 100 \mathrm{sccm}$ (4 / $0.5 \mathrm{sec}$ ), Throttle 15\% ( $\rightarrow$ Pressure: 8.4Pa), CCP 100W (chopped $20 \mathrm{~ms}$ on $/ 80 \mathrm{~ms}$ off), SH: $110 \mathrm{~mm}$, Temperature: $-120^{\circ} \mathrm{C}, \sim 1 / 2 \%$ load, Duration $10 \mathrm{~min}$

We explored a second method in which a negative tapered profile might be obtained; the image force (IF) effect. The IF effect is caused by the electrostatic attraction of incoming ionic species due to influencing fields, towards the silicon sidewalls as shown in Figure 4-6. This force is inversely proportional to the square of the distance, so the acceleration toward the sidewall is ever increasing until the particle collapses with the surface causing a negative taper. The lower the bias, the slower the ionic species move, the more they will deflect and thus the more negative the taper. A high speed etch recipe based on cryogenic $\mathrm{SF}_{6} / \mathrm{O}_{2}$ is used to show the effect of the IF [6]. This kind of recipe is known for its thin $\mathrm{SiO}_{\mathrm{x}} \mathrm{F}_{\mathrm{y}}$ passivation layer which makes it sensitive for the IF when a low bias voltage is used. From the resulting etch profile as shown in Figure 4-6 one can observe two important effects. The upper part of the profile is insufficiently passivated and therefore the silicon is etched by radicals. Second, the negativity of the taper is ever increasing towards the bottom of the profile. This is an undesired property for the diverging part of the nozzle because it results in flow separation when the angle becomes too large. There is no process setting found that result in a proper negative taper while the upper part of the profile is preserved. 


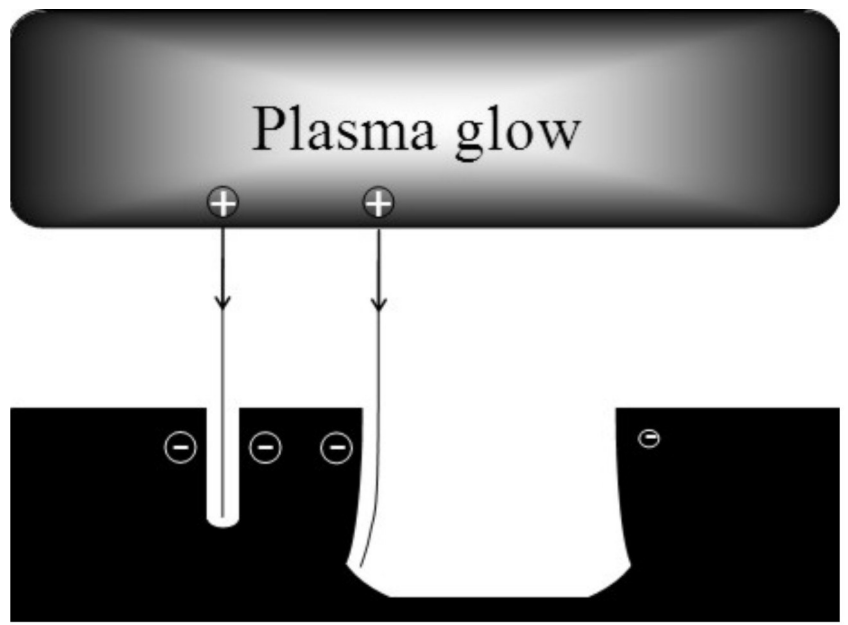

a)

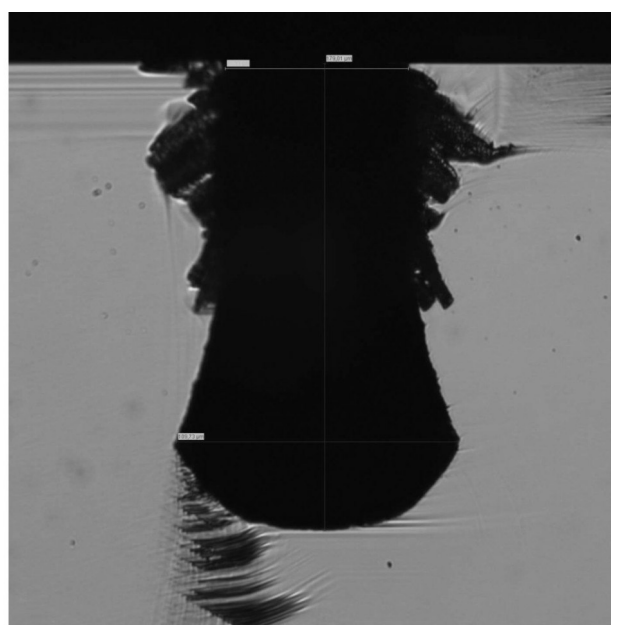

b)

Figure 4-6: a) IF effect: image force caused by deflection of ions inside trenches. b) Mixed process settings: $\mathrm{SF}_{6}+\mathrm{O}_{2}: 400 \mathrm{sccm}+27 \mathrm{sccm}$, Throttle 15\% ( $\rightarrow$ Pressure: 6.4Pa), CCP 20W (chopped 20ms on/80ms off), SH: $150 \mathrm{~mm}$, Temperature: $-120^{\circ} \mathrm{C}, \sim 1 / 2 \%$ load, Duration $10 \mathrm{~min}$

A sufficiently negative tapered profile seems to be difficult to obtain with DRIE. Another approach to make the nozzle shape might be to use a hole with a positive taper. Although we did not do in depth studies into this we discuss four methods where positive tapered holes are made from both sides of the wafer to form the nozzle.

The first option is to tune the DRIE process in such a way that, instead of a negative taper, a positive taper is obtained. This can be done by enhancing the passivation, by increasing the flow of the etch inhibiting gas or by increasing the time of this passivation step. The drawback of this approach is the forming of black silicon when the etch inhibiting layer is not totally removed from the bottom of the etching feature. Increasing the bias voltage would help but is destructive for the etch selectivity. A positive taper of $15^{\circ}$ or higher without the forming of black silicon and enough selectivity is therefore probably impossible.

The second method is gray-scale lithography [7]. By gray-scale lithography a variation in the thickness of the photosensitive mask layer is obtained (Figure 4-7.a). When the etch selectivity between silicon and photoresist is known one can tune the etch profile in the silicon by the thickness variation in the resist layer. The footprint in the silicon correlates directly to the variation in resist thickness amplified by the etch selectivity as is depicted in Figure 4-7. For a positive taper with an angle of $15^{\circ}$ a very low selectivity is required. This means, one needs a very 
thick layer of photo-resist to be able to etch through a silicon wafer. This is impractical and thus this method is not an option.

As a third method we consider the use of the RIE-lag effect and thereby obtain the positive tapered profile [6]. The RIE-lag is caused by the difference in etch speed of small sized features compared to larger sized features. In Figure 4-8.a the mask is shown which is used to show the RIE-lag effect; the black circles are etched. An example of the RIE-lag effect is shown in Figure 4-8.b. The trenches at the outer side of the etched profile are $2 \mu \mathrm{m}$ and etching slower than the hole in the middle which has a much larger diameter. A stepwise profile is obtained by this method and is therefore not very suitable for making a nozzle because a smooth profile required.

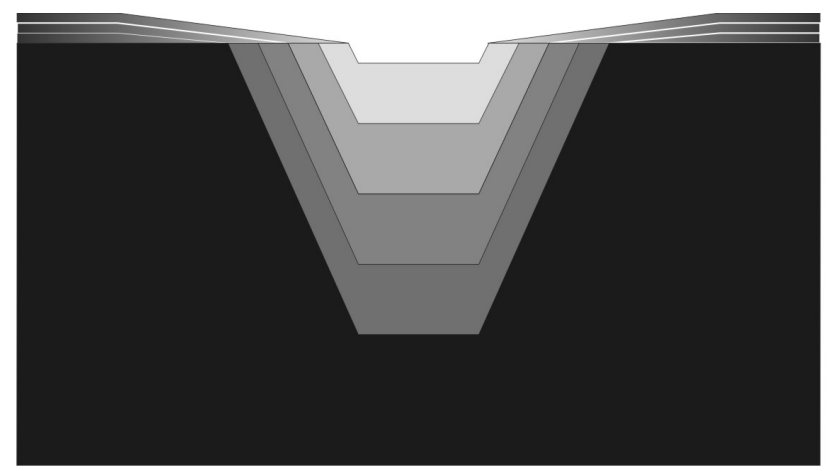

Figure 4-7: Development of positive tapered hole with Grey-scale lithography.

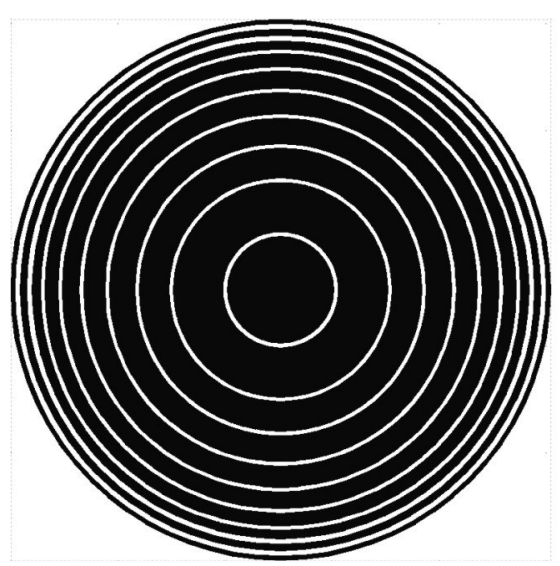

a)

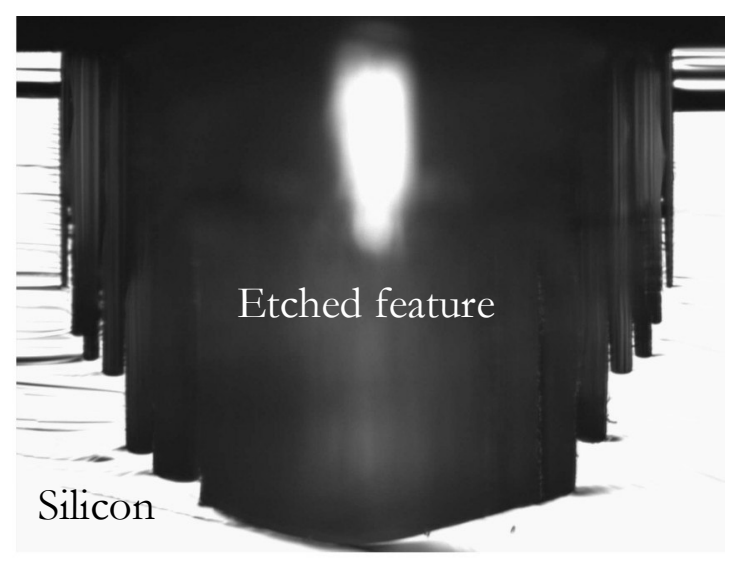

b)

Figure 4-8: a) Mask used to show RIE-lag effect. b) Example of the RIE-lag effect. 
The fourth possibility is to use a DC-bias voltage to obtain a positive tapered profile [4]. In a DC biased discharge, the electron and ion currents through the dark space are not equal, which will cause charge accumulation on an insulating mask. A resist pattern will charge negatively with respect to the plasma in order to draw zero net current, while the exposed conducting areas of the wafer will draw a net current. The resist layer will charge positively with respect to the under lying Silicon and thereby, the electric field lines in the plasma sheath are changed at the edges of an etched feature in the vicinity of the resist pattern. The distorted electric field causes the ions to deflect away from the charged resist layer and changes the etching profiles. In Figure 4-9, this distortion results in convex etched walls which bulge strongly towards the interior on an etched groove.

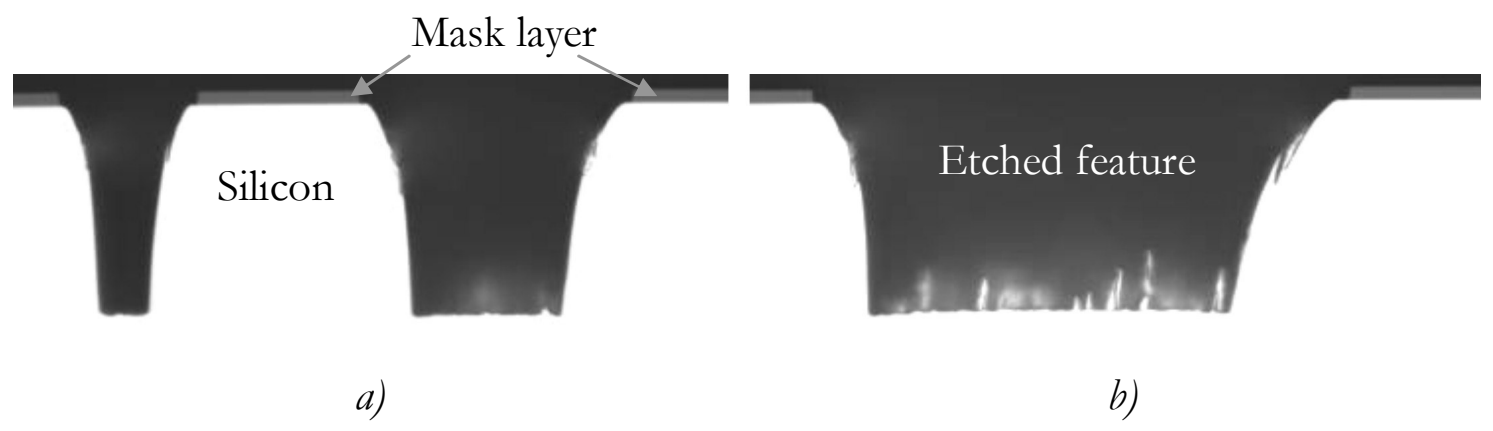

Figure 4-9: Positive taper by DC-plasma a) small convex etched walls b) asymmetric etched feature

The distortion of the field lines depends on the shape and area size of the neighboring resist pattern. An irregular pattern can result in an asymmetrically etched feature as is shown in Figure 4-9.b. The shape of the hole can be tuned by the design of the resist pattern and the strength of the DC-bias voltage. But again, with this method the profile at the exhaust changes quickly, increasing the risk of rupture of the boundary layer and thus difficult to control thrust power. 


\subsubsection{Femtosecond laser machining}

For the second method of fabrication femtosecond laser machining (FLM) is explored, which is considered a promising technology for manufacturing down to the micro-scale. Due to ultra short pulses and high peak intensities a small amount of material is ablated resulting in high accuracy and repeatability $[8,9]$.

In order to machine the nozzle holes with diameters of $230 \mu \mathrm{m}$ the laser spot is manipulated over this area in a specific machining pattern. To obtain the conicaldivergent nozzle shape the silicon disc is treated from two sides. From the topside, the converging part, a hole is drilled through the wafer. From the backside the point of penetration is used to align the silicon wafer for a second treatment to obtain the diverging part of the nozzle.

The energy density (fluence) in the focused laser spot of ø $25 \mu \mathrm{m}$ is $0.81 \mathrm{~J} / \mathrm{cm}^{2}$ (Gaussian distributed). This is substantially above the minimum energy density needed on the surface of the material for ablation to occur, i.e. the ablation threshold. We found that the minimum beam fluence needed for ablation to occur depends on the machining speed and pulse repetition rate. At a machining speed of $80 \mathrm{~mm} / \mathrm{s}$ and pulse repetition rate of $50 \mathrm{kHz}$ the ablation threshold is determined at $0.18 \mathrm{~J} / \mathrm{cm}^{2}$. To machine the nozzles, a machining pattern was programmed to manipulate the laser over a circular area. This pattern and the configuration of the laser settings were optimized for sidewall smoothness and machining time.

The slope of the wall is implicitly controlled by the energy density. The relationship between the fluence used for machining the holes and the threshold fluence determines the naturally occurring slope of a side wall. This is due to the fact that the laser energy irradiates a larger area on a sloped wall, as depicted in Figure 4-10. Once this area gets too large, at a certain slope, the irradiated fluence is not high enough to overcome the ablation threshold and no material is removed.

This effect is used to tune the angle of the side wall by adjustment of the fluence rather than adjusting the machining pattern. The successive scans over the sloped sidewalls lead to formation of a very thin melt layer that improved the smoothness of the sidewalls with every overscan. 


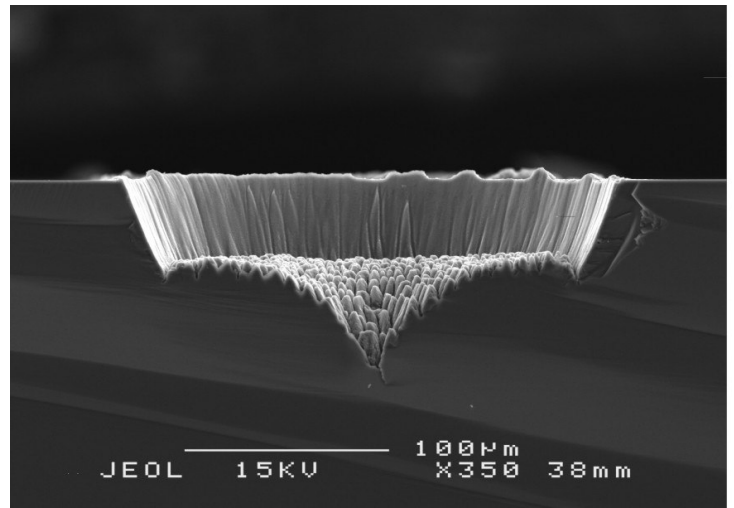

a)

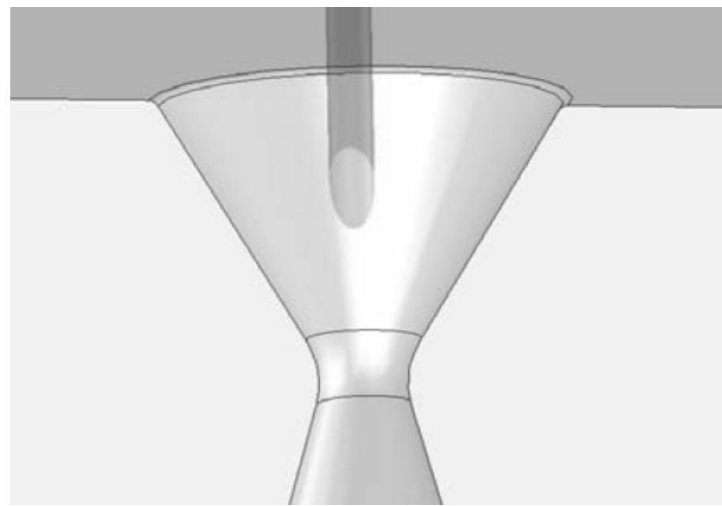

b)

Figure 4-10: a) SEM image of unfinished hole, b) increased irradiated area

The limit for the slope of the wall is given by the maximum available pulse energy. This leads to a minimum half angle (theta) of $15^{\circ}$. This slope could be further reduced by using lasers with higher pulse energies or by tighter focusing, in order to meet the requirements for the diverging half angle. In principle an angle could be adjusted also to very large values, just by choosing the machining fluence close to the threshold fluence. A practical drawback would be the long machining times. The converging half angle of $20^{\circ}$ is easily attained; however this was not performed in the scope of these experiments. The location of the nozzle throat is controlled by adjusting the begin diameter of the holes drilled from the two sides.

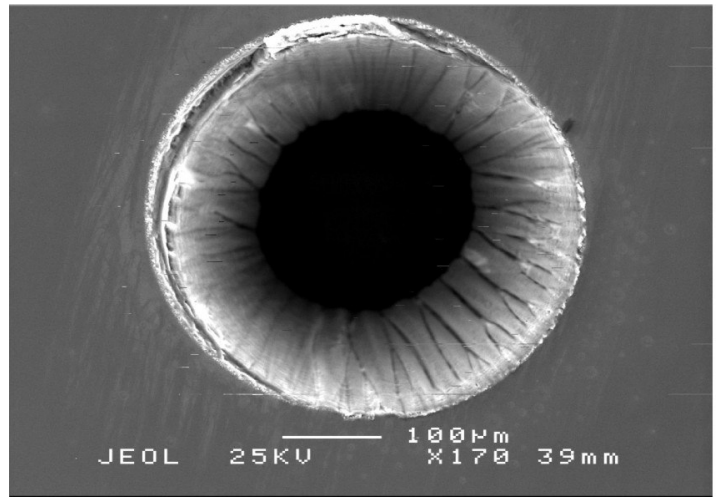

a)

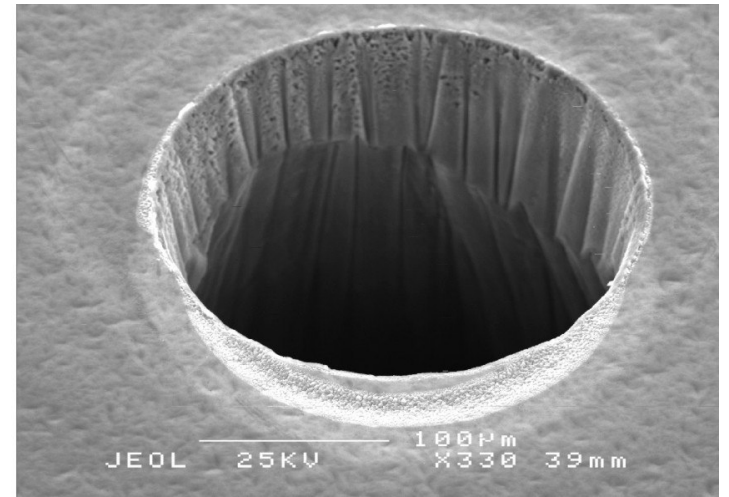

b)

Figure 4-11: SEM images: a) nozzle inlet b) nozzle outlet 


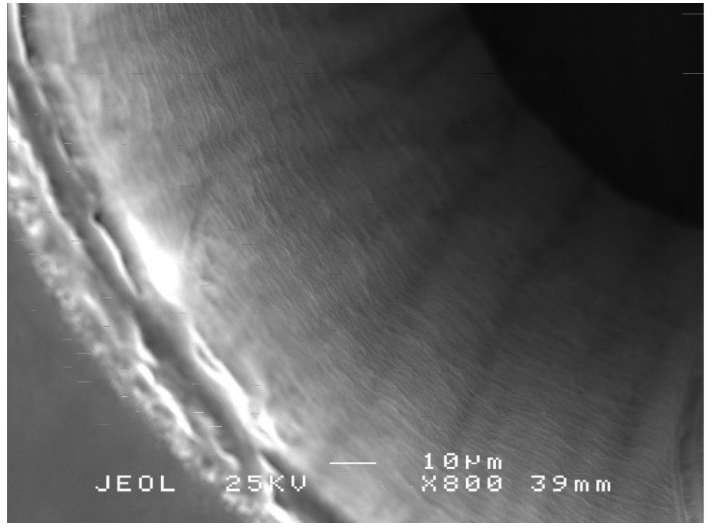

a)

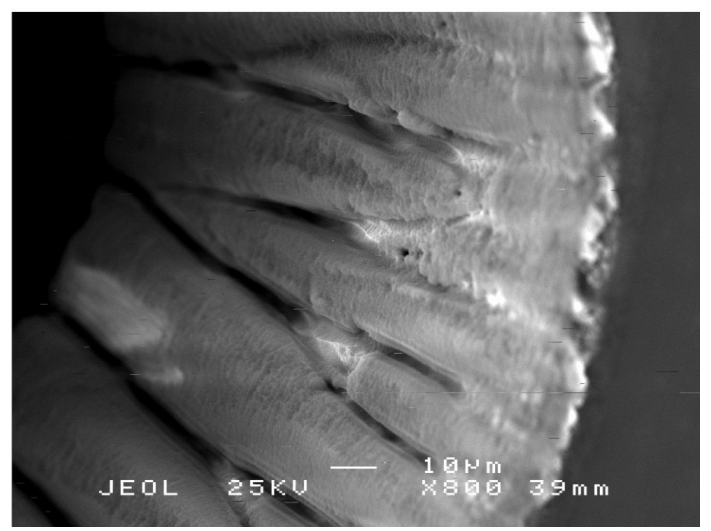

b)

Figure 4-12: SEM images: a) Zoom of Figure 4-10.a left under b) Zoom of Figure 4-10.b right under

Figure 4-11 shows a nozzle inlet and outlet of a typical nozzle produced with a throat diameter of $210 \mu \mathrm{m}$. The figures show that the throat of the nozzle is not exactly aligned in the middle and is therefore slightly elliptical. This is attributed to little misalignment of the two holes that are laser machined from both sides of the wafer. The sidewall of the nozzle locally shows small trenches as is visible in Figure 4-12. a and $\mathrm{b}$. This is a residual of the micro columns which are formed during laser treatment. The trenches are more pronounced in Figure 4-12.b compared to Figure 4-12.a. It is hypothesized that this is caused by an elliptical energy distribution in the laser spot.

New developments aim at improved control over the geometry of drilled holes and nozzles. For this, a new focusing and manipulation technique is developed, which enables the explicit definition of nozzle geometries. 3D shapes can be determined directly in software instead of using the ablation threshold to control the converging angle. First results on metals are conducted as presented in Figure 4-13. Here a smaller focal spot size was used, enabling the machining of holes down to 1 $\mu \mathrm{m}$. This new technique is not yet applied on the machining of nozzles for this particular application. 


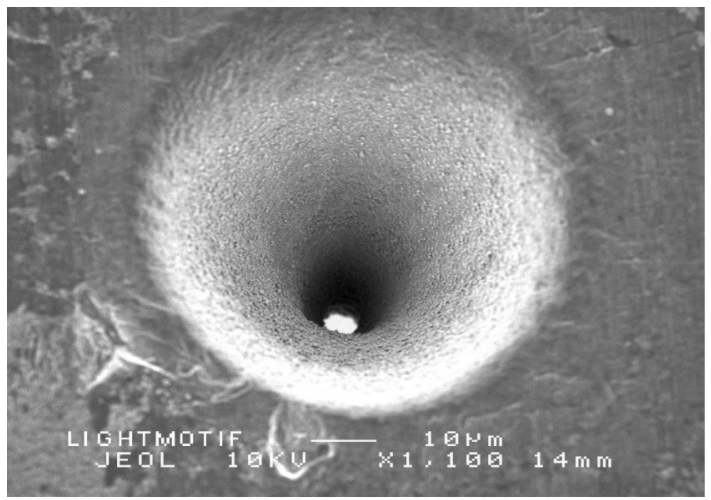

a)

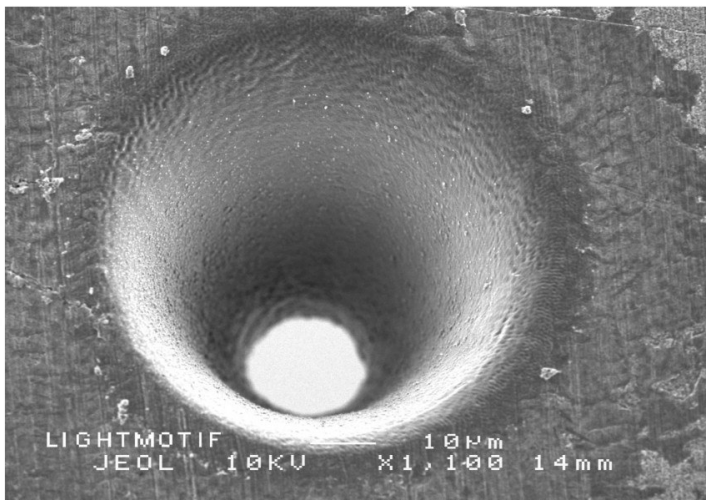

b)

Figure 4-13: Hole in silver sheet with $1 \mu \mathrm{m}$ laser spot

The nozzles that are fabricated by FLM have been measured for their performance at a test stand at the Delft University [10]. The test stand is designed for thrust measurements in atmospheric conditions in the range of $20-1000 \mathrm{mN}$.

Nozzles that are supposed to be used in space require a different expansion ratio than the nozzles that are tested under atmospheric circumstances. At sea level the nozzle expansion ratio should be chosen in such a way that the pressure at the exit of the nozzle does not drop below the atmospheric pressure [11].

A limitation of the test stand is reflected in the fact that thrust values below $20 \mathrm{mN}$ can not be measured accurately. For the formation flying of two Cubesats a thrust between 1 and $10 \mathrm{mN}$ is preferred which can not be measured accurately with this test stand. To test the performance of the nozzles, two arrays of nozzles have been designed and manufactured. An area ratio between the exit and the throat of 1.2 is selected to avoid over expansion which can result in flow separation. A single nozzle is designed to deliver $5 \mathrm{mN}$ at 2 bar inlet pressure (1 bar differential pressure). Such a nozzle has a throat diameter of $210 \mu \mathrm{m}$ and an outlet diameter of $230 \mu \mathrm{m}$. Two nozzle arrays having a hexagonal pattern were machined; one with 19 (Figure 4-14.a) nozzles expecting to deliver $95 \mathrm{mN}$ thrust. The second array has 7 nozzles which should result in $35 \mathrm{mN}$. Another nozzle disk was fabricated with only 1 nozzle for $5 \mathrm{mN}$ thrust. A glass tube is fusion bonded onto the silicon nozzle discs and a fine machined threaded metal part is attached to the tube with epoxy glue. The resulting nozzle assembly is shown in Figure 4-14.b. This assembly is connected to the plenum, which will finally contain the cold gas generators, but for 
this test, is only equipped with a pressure sensor and a gas inlet. This gas tank, which is manufactured by TNO, is positioned in the test stand as shown in Figure 4-15.

To be able to compare the measurements with the theory the throat and outlet diameter of the nozzles have been measured. The dimensions of the individual nozzles of the 19 nozzle array have been taken from SEM images to give an indication of the reproducibility of the fabrication process. A typical manufactured nozzle has a throat diameter of $219 \mu \mathrm{m}$ and an exit diameter of $259 \mu \mathrm{m}$. Since the nozzle throats are slightly elliptical the given numbers are a mean value. The nozzles are bigger than the required $210 \mu \mathrm{m}$ and $230 \mu \mathrm{m}$ for the throat and the outlet diameter, respectively.

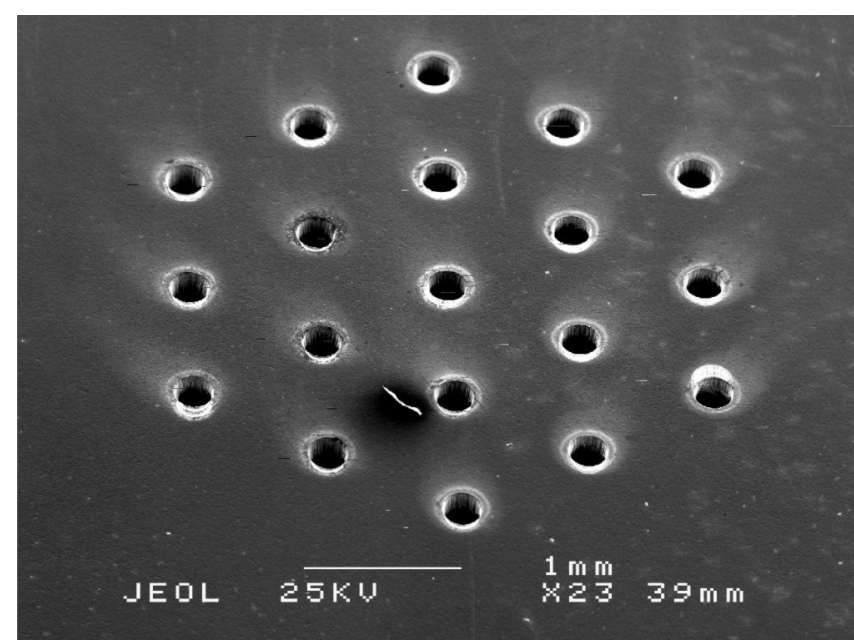

a)

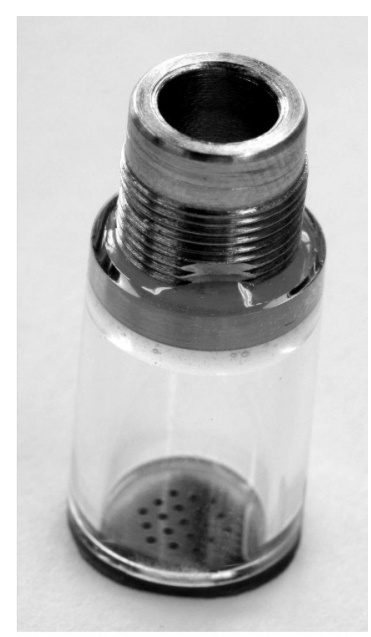

b)

Figure 4-14: a) SEM image of the 19 nozzle array b) Nozzle assembly

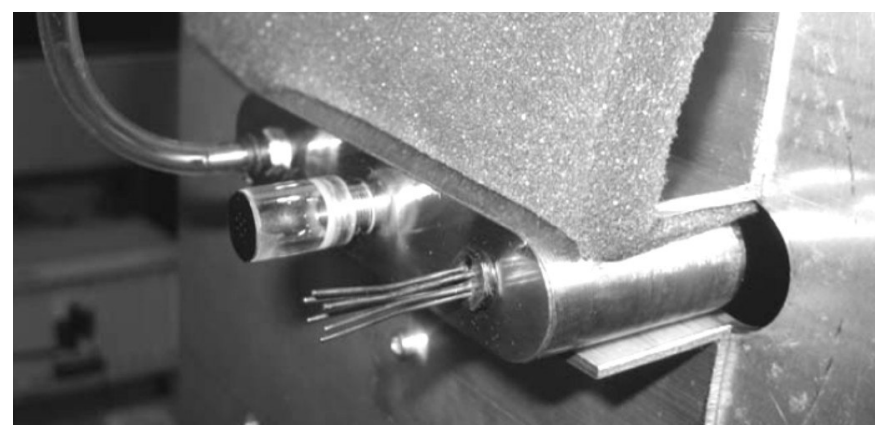

Figure 4-15: Assembly of the nozile unit, plenum, pressure sensor and gas inlet on the test stand [10] 
Measurement of the individual nozzles indicates that differences in throat area from one nozzle to the other are within $10 \%$. It must be noted that the measurement method is not exact due to low resolution SEM images, but it gives an indication of the big differences in throat area. This difference is attributed to the error in double sided alignment. The first hole is drilled through the silicon wafer. The point of puncture of this first hole is used for the alignment of the second hole, which is laser machined from the other side of the wafer. This alignment is critical to obtain a symmetrical nozzle and should be improved considerably. For lithography there are optimized machines which can align with a precision of typically $1 \mu \mathrm{m}$. Positioning systems for laser machining are not optimized for double sided positioning within $1 \mu \mathrm{m}$ precision. A good method might be to use infrared light, for which silicon is transparent, to align to machined structure on the backside of a wafer.

An extensive description of the measurements is given by Zandbergen and can be found in [10]. It is shown that the mass flow through the nozzles is in linear relation with the inlet pressure. This is expected from the theory as described in chapter 2. The measured thrust levels, range from $\sim 4 \mathrm{mN}$ for the disc with only 1 nozzle at a plenum pressure of $2 \mathrm{bar}$, to over $300 \mathrm{mN}$ for the disc with an array of 19 nozzles at a pressure of $\sim 5$ bar. The ratio for the thrust between the 1,7 and 19 nozzle discs is $17: 7.7: 1$. For identical nozzles we expect a ratio between the thrust levels which is identical to the number of nozzles. The deviation of the thrust ratio is mainly attributed to the differences in the dimensions of the nozzles. Furthermore, the large inaccuracy in the measurement of the 1-nozzle disc is adding to the error. The measurement accuracy is within $\pm 2 \mathrm{mN}$ in this low thrust range which is enormous for a measured thrust of $4 \mathrm{mN}$.

For future measurements it is important that the dimensions of all individual nozzles are accurately known. Furthermore, the test stand should be improved to allow for higher accuracy in the low thrust range by reducing the signal noise. 


\subsubsection{Powder blasting and heat treatment}

The final fabrication method that is explored is a method for making a nozzle shape in Pyrex glass. Powder blasting is used to make a hole in the glass. It is a physical process where energetic particles impact the glass substrate causing erosion. A beam of $\mathrm{Al}_{2} \mathrm{O}_{3}$ particles is expelled and manipulated over the work-piece. The cross-section of a powder blasted hole has a rounded V-shape, which is caused by the typical impact angle dependent removal rate for brittle materials [12]. Although in some application this inclined sidewall is an unwanted effect, we will use this property to tune the angle of the diverging part of the nozzle.

Figure 4-16 shows cross-sections of the development of a hole. From left to right the duration of the treatment is increased, i.e. the number of times the abrasive beam of $29 \mu \mathrm{m} \mathrm{Al}_{2} \mathrm{O}_{3}$ particles is manipulated over a particular hole.
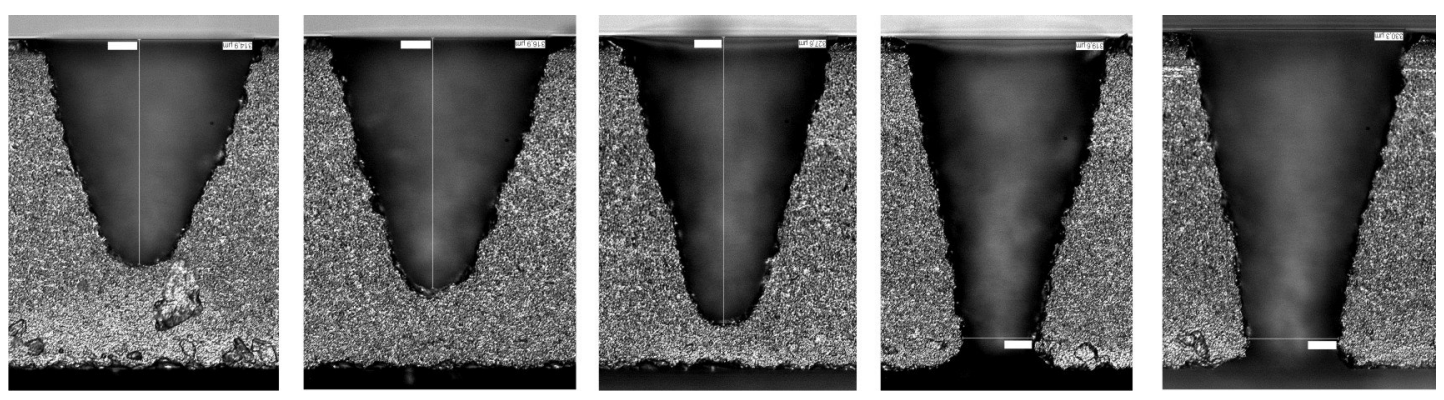

Figure 4-16: $300 \mu m$ holes, increasing treatment duration
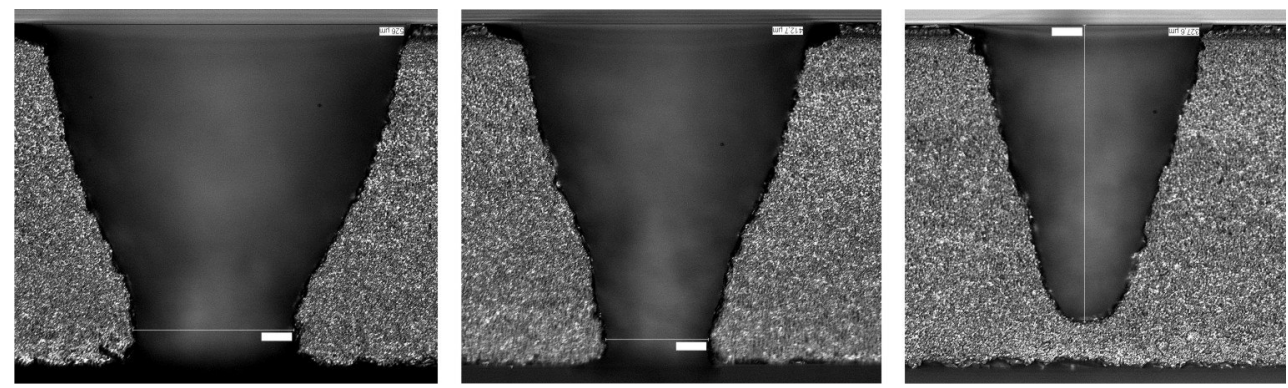

Figure 4-17: Holes with different mask openings

The shape of the hole changes from parabolic to a straight inclined sidewall at a certain aspect ratio as can be seen in Figure 4-17. For the nozzle, we aim at a straight sidewall but a bell shaped nozzle can be obtained. 
When smaller particles are used a steeper sidewall can be obtained and the characteristic shape of a straight inclined sidewall is found at higher aspect ratios. The angle that could be obtained with the $29 \mu \mathrm{m}$ particles is in between $14^{\circ}$ and $20^{\circ}$ depending on the duration of the treatment. For a larger hole longer treatment is required to obtain a straight inclined sidewall meaning the selectivity of the mask material towards the Pyrex glass is the limiting factor.
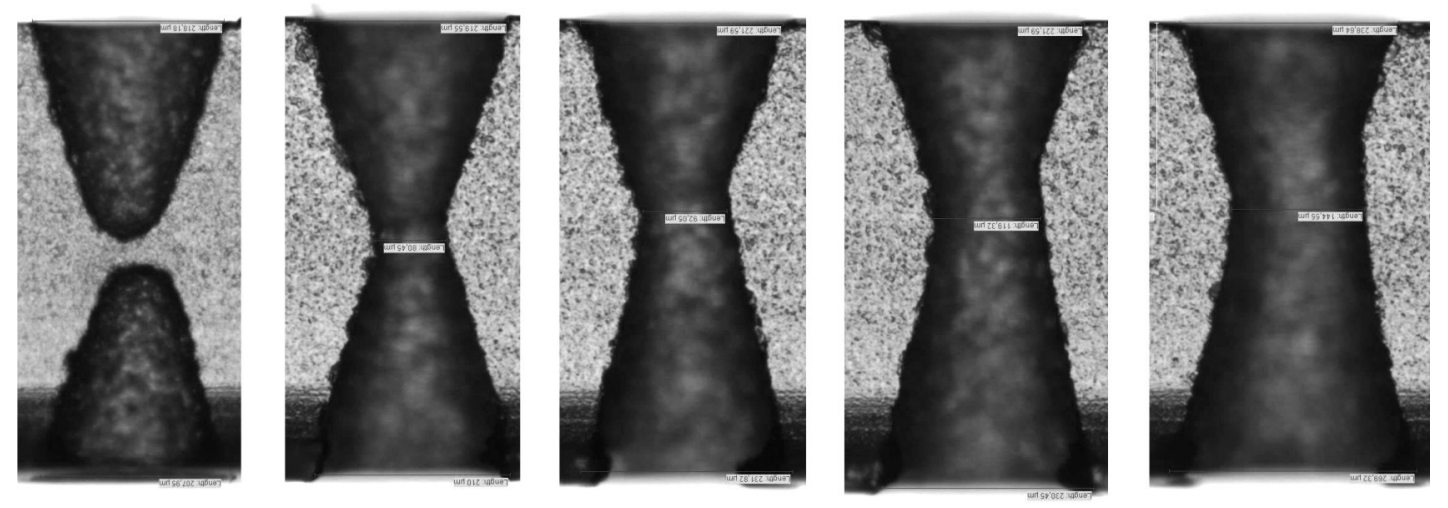

Figure 4-18: Treatment from two sides

First attempts to obtain the conical converging-diverging nozzle where done by treatment of the Pyrex glass wafer from two sides. The result is shown in Figure 4-18. The glass is treated from the top for 4 times. From the backside the glass is treated 4 times (the most left picture) up till 8 times (right). It is observed that the holes are a little bit misaligned, which is an issue of interest because of the thrust vector. With increased treatment the nozzle throat becomes wider and it shifts upwards. When the angle of inclination is known for a certain process setting the nozzle shape can be predicted for a certain inlet and outlet diameter and is tuned by the duration of the treatment.

A big disadvantage of powder blasting is the high surface roughness which degrades the performance of the nozzle. The smaller the particles which are used in the abrasive process the more smooth the resulting sidewall. For $29 \mu \mathrm{m}$ particles the resulting roughness is in the order of $R_{a} 2.5 \mu \mathrm{m}$ and for $9 \mu \mathrm{m}$ particles it is approximately $\mathrm{R}_{\mathrm{a}} 1.2 \mu \mathrm{m}$ [13]. One could argue to use smaller particles to obtain a smoother result, were it not for that the size of the particles also determines the angle of inclination and smaller particles result in an undesirable low angle. Another 
possibility to make the surface of the sidewall smoother is to introduce a high temperature anneal [13]. It is know that in this way the roughness can be reduced to $\mathrm{R}_{\mathrm{a}} 0.5 \mu \mathrm{m}$. Such a high temperature step is required anyway for the integration of the nozzle with the MST feeding and thruster system. By means of the high temperature step a glass machined tube is bonded to the MST part consisting of a valve and the nozzle. The glass tube is functioning as a hermetically sealed package, fluidic interconnect as well as a macro support for the fragile MST component [2]. This means the glass nozzle undergoes a temperature step of $790^{\circ} \mathrm{C}$. Figure $4-19$ shows a nozzle after heat treatment. The nozzle sidewall is indeed smoothened. We also see that the sharp corners at the inlet and outlet are rounded, caused by surface relaxation. The rounding of the diverging part of the nozzle is an undesirable effect. The diverging angle increases towards the nozzle exit which causes flow separation and thus sacrificing thrust efficiency.

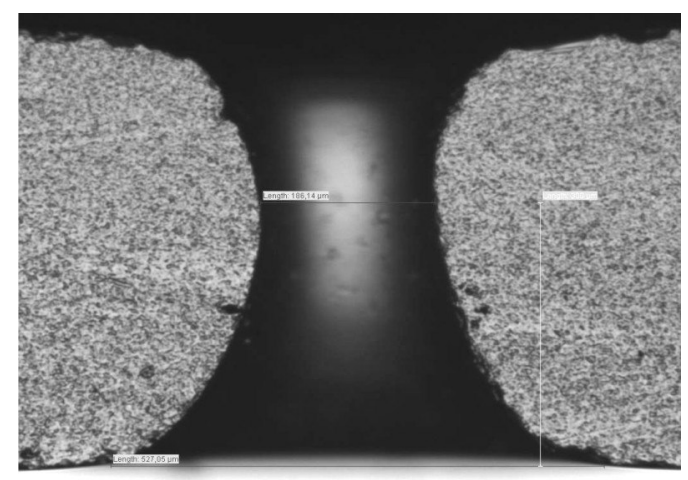

Figure 4-19: Nozzle after heat treatment

The rounding of the corners by means of a high temperature treatment can be used in our advantage when we make sure that the shape of the diverging part of the nozzle is fixed and the entrance of the nozzle is free to relax its corners.

The fabrication scheme for the manufacturing of a nozzle by a combination of powder blasting and heat treatment is shown in Figure 4-20. After applying a mask with circular features with a diameter of $400 \mu \mathrm{m}$, a hole is made through a $500 \mu \mathrm{m}$ thick Pyrex glass wafer by powder blasting. The novelty of the process lies in the following two steps. First a silicon wafer is bonded to the Pyrex wafer to fix the exit diameter during a consecutive heat treatment. This anodic bonding step is done at a temperature of $450^{\circ} \mathrm{C}$. At this temperature the Pyrex glass keeps its shape. Second, 
a high temperature step is done to round of the corners at the entrance of the nozzle without rounding of the exit of the nozzle [14]. Hereby the final nozzle shape is obtained. In the last step the nozzle exit is opened by etching a hole through the silicon wafer with reactive ion etching.

1

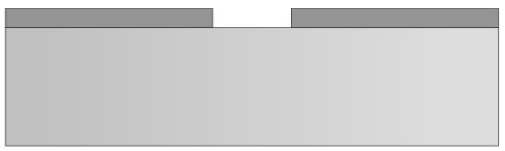

2
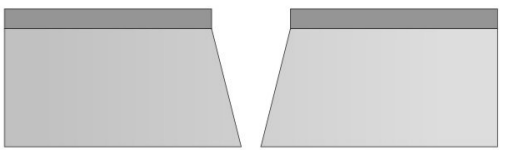

3

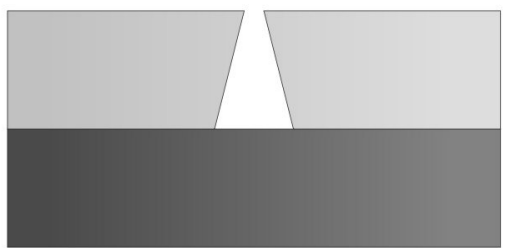

4

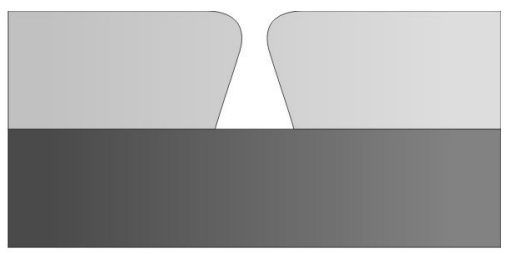

5
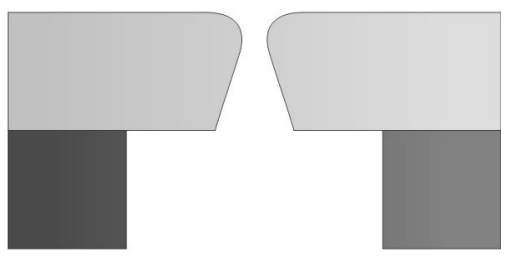

Figure 4-20: Glass nozzle fabrication scheme

Figure 4-21 shows a cross-section of the resulting nozzle shape. The throat diameter of this nozzle is $150 \mu \mathrm{m}$ and the radius of curvature and the position of the throat are controlled by the heat treatment. The half angle of the nozzle is approximately $20^{\circ}$ but can be tuned for smaller angles.

The serrated contour of the nozzle that is seen in Figure 4-21 is the result of the dicing; it is not the roughness of the nozzle surface itself. The surface of the nozzle inlet and throat are improved considerably by the high temperature step. A nozzle inlet is shown in Figure 4-22.a. One can observe some irregularities in the surface that might be caused by dirt particles or residual particles of the powder blasting process. It is difficult to give an estimated number for the roughness from these SEM pictures but it is much less than $1 \mu \mathrm{m}$. In Figure 4-22.b a magnification of the nozzle exit is displayed. It is observed that there is sudden increase in surface roughness at the outlet of the nozzle. The approximated roughness in this part of the nozzle is in the order of microns but this is relatively harmless for the thrust efficiency. More nozzles are presented later on which do not have this sudden change in roughness. 
The smallest mask opening that can be used to powder blast through a wafer of $500 \mu \mathrm{m}$ thickness is $250 \mu \mathrm{m}$ in diameter when assuming an inclined angle of $14^{\circ}$. This means that the exit of the nozzle cannot be smaller than $250 \mu \mathrm{m}$. When using a thinner wafer the exit diameter can be reduced. However, this lowers the outlet to throat area ratio and therefore reduces thrust efficiency.

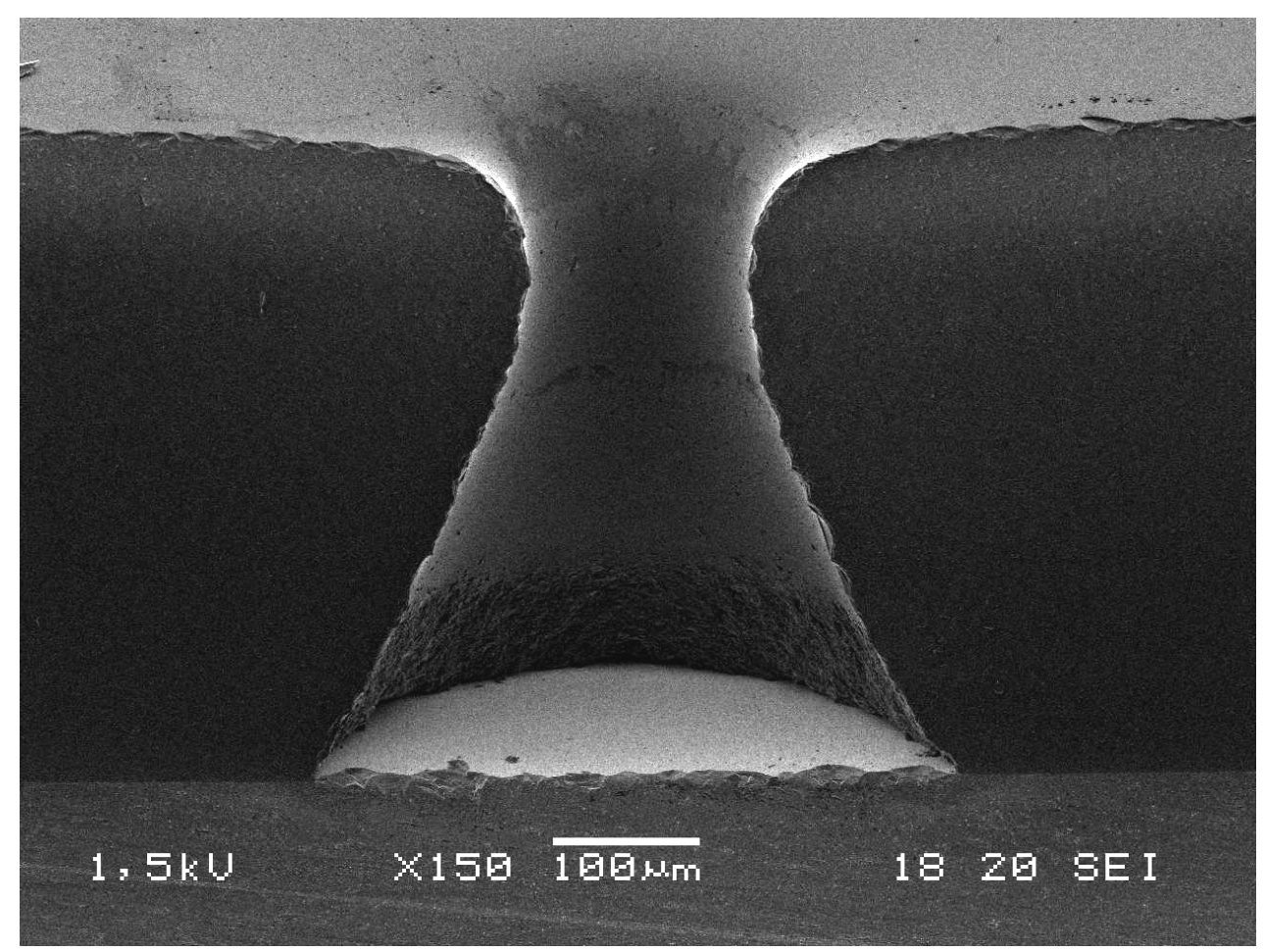

Figure 4-21: Cross-section of a glass nozzle

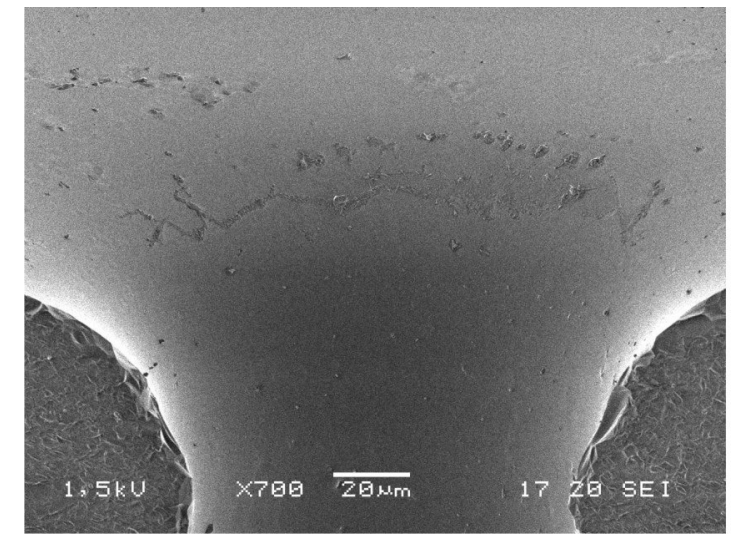

a)

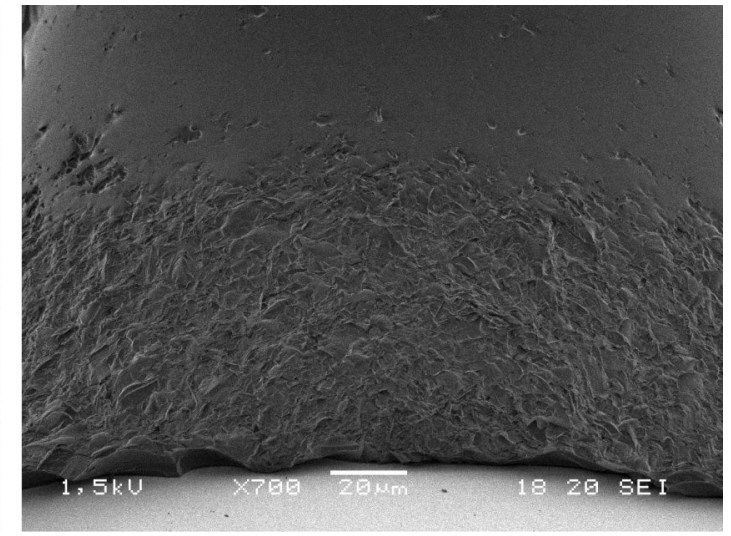

b)

Figure 4-22: a) Zoom in on nozzle inlet. b) Zoom in on nozzle outlet 
Nozzles have been fabricated by the powder blasting and heat treatment method, which are supposed to fly on the Delphi n3Xt micro satellite. This is a micro satellite under development by the University of Delft, the Netherlands. The micro satellite measures $10 \mathrm{~cm} \cdot 10 \mathrm{~cm} \cdot 30 \mathrm{~cm}$ and is supposed to be launched in 2010 .

Several nozzles have been fabricated with different outlet diameters. The outlet diameters are $280,300,320,340,360,400$ and $500 \mu \mathrm{m}$, which is expected to result in throat diameters ranging from 70 to $240 \mu \mathrm{m}$. Different sizes have been made for testing purposes. For the actual mission we aim at the nozzle with an outlet of $300 \mu \mathrm{m}$ and an expected throat diameter of $100 \mu \mathrm{m}$. The nozzle is positioned in the center of a glass disc with a diameter of $6 \mathrm{~mm}$. During the heat treatment, to obtain the final nozzle shape, glass tubes are bonded to the glass discs. The fabrication process of these nozzles differs at two points from the process described in Figure 4-20. First, instead of Pyrex glass, Borofloat glass is used which is very similar in composition. Second, the anodic bond step is done in a dedicated machine whereas for the previous nozzles it was done manually in a furnace. The anodic bonding process in the dedicated machine is optimized to result in the best bond. Furthermore, the abrasive beam of $\mathrm{Al}_{2} \mathrm{O}_{3}$ particles is precisely positioned at right angle of the wafer surface. This is important to obtain a symmetrical hole and thus a symmetrical final nozzle shape. The resulting nozzles are shown in Figure 4-23. From these cross sections it is observed that the smaller nozzles have a smaller curvature at the inlet. It is expected that this rounding of the nozzle inlet can be tuned by the heat treatment. In Figure 4-24 the top view of a nozzle with an outlet of $320 \mu \mathrm{m}$ - as defined by lithography - is shown. Figure 4-24.a shows the nozzle before the heat treatment. The picture is taken from the inlet of the nozzle and has a mean diameter of $148 \mu \mathrm{m}$. The chipping of the glass is clearly seen and it is also observed that the hole is not perfectly circular. Figure 4-24.b shows the same nozzle after the heat treatment. The picture is again taken from the inlet of the nozzle and the focus is on the throat which has a mean diameter of $121 \mu \mathrm{m}$. The diameter of individual nozzles shrinks $27 \mu \mathrm{m}( \pm 5 \mu \mathrm{m})$ due to the heat treatment. When comparing the shape of Figure 4-24.a and Figure 4-24.b it is observed that most bumps have disappeared after the heat treatment resulting in an almost perfect circular shape. 


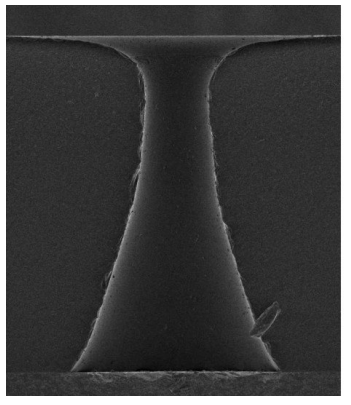

a) 280

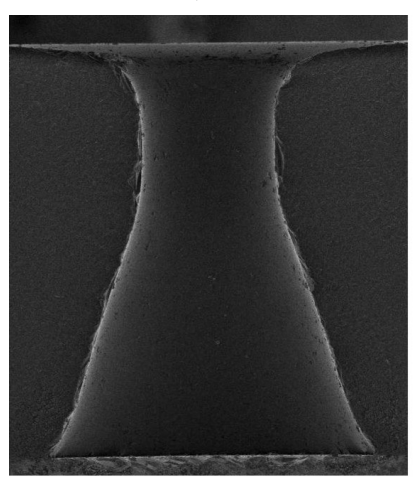

e) 360

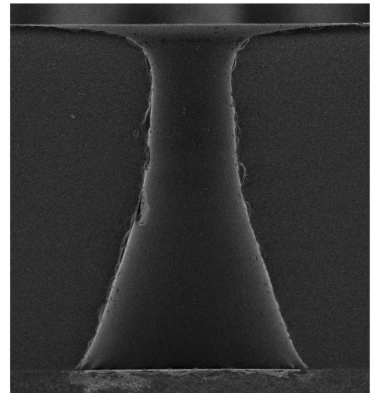

b) 300

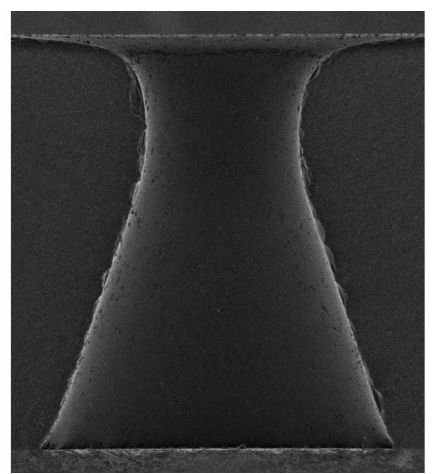

f) 400

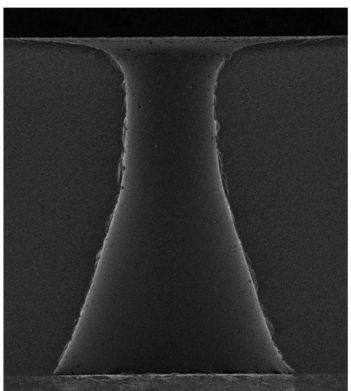

c) 320

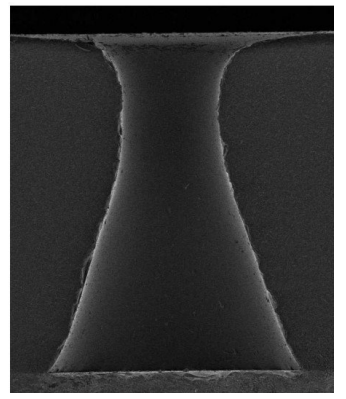

d) 340

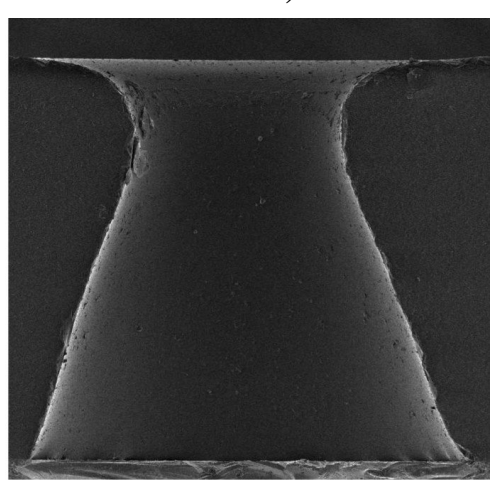

g) 500

Figure 4-23: SEM pictures of the resulting noziles. The numbers indicate the outlet diameter (as defined by lithography).

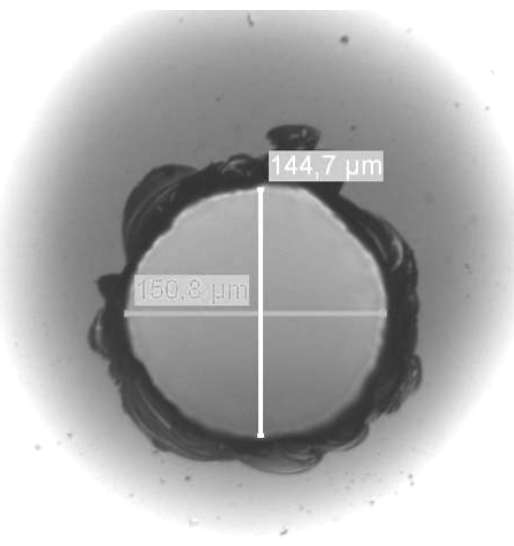

a) 320

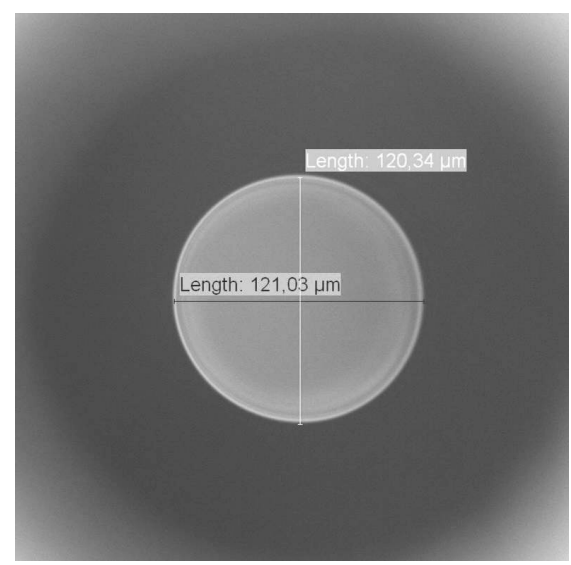

b) 320

Figure 4-24: a) bole after powder blasting (from inlet) b) bole after heat treatment (focus on the throat) The numbers indicate the outlet diameter (as defined by lithography). 
In Figure 4-25.a a SEM picture is shown which is taken from the outlet. It is the same nozzle as in Figure 4-25.b. When looking at the outlet of the nozzle one can see that the shape is irregular. The outlet of the nozzle is inhibited to reflow due to the silicon wafer that is attached to the outlet by anodic bonding.

Figure 4-22.b the outlet of a nozzle is shown. It was observed that the outlet is rougher than the surface in the throat. Figure 4-25.b shows the outlet of a nozzle which is made with the minor adjustments here presented. Now the outlet of the nozzle is as smooth as the nozzle throat. Since the properties of Pyrex and Borofloat glass are comparable it is expected that this difference is due to the difference in the anodic bonding step. During anodic bonding $\mathrm{Na}+$ ions are drawn away from the interface between the glass and silicon. The depletion of $\mathrm{Na}+$ ions changes the physical properties of the glass. This might cause a higher viscosity and thus less reflowing of the glass. In the controlled and optimized bonding process a lower voltage and a shorter process time are used which is expected to result in less depletion of $\mathrm{Na}+$ ions. It is hypothesized that the physical properties of the glass change much less resulting in comparable roughness over the whole nozzle surface.

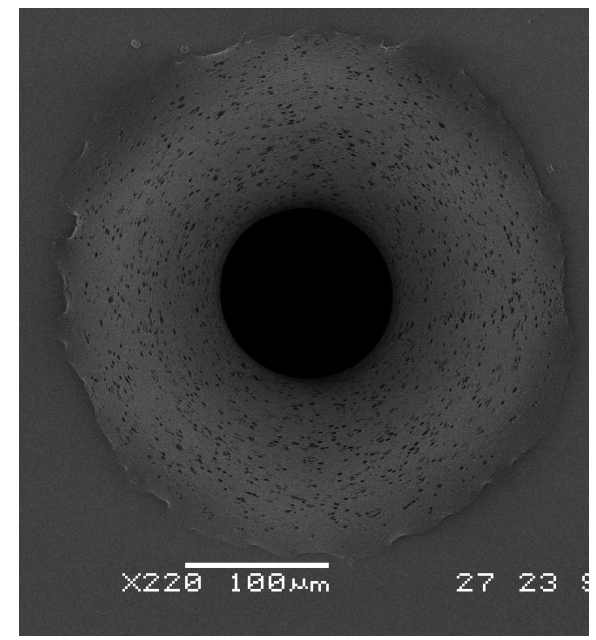

a)

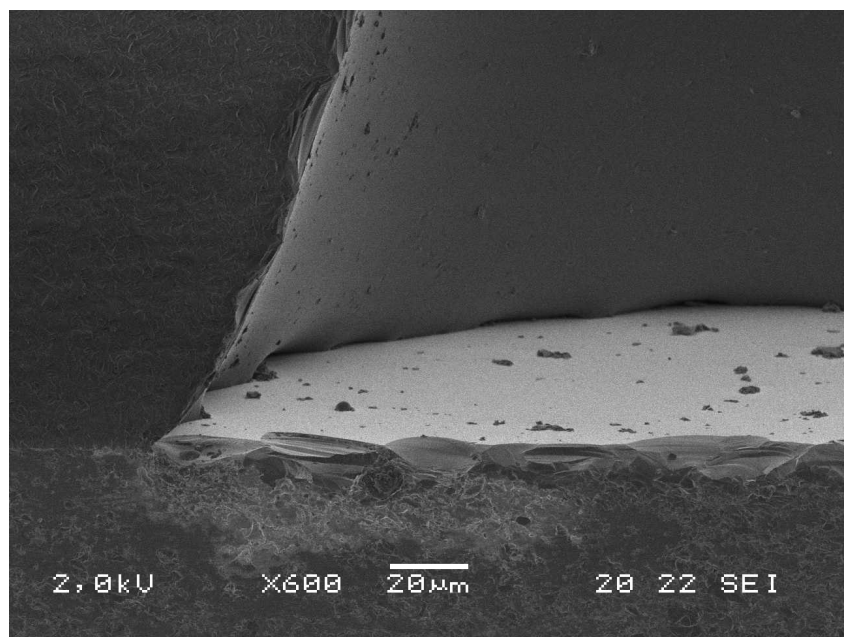

b)

Figure 4-25: a) SEM image of final nozzle (from outlet) b) SEM image of the nozzle outlet 


\subsection{Discussion and conclusion}

Nozzles manufactured by different technologies are compared. Table 4-I displays the pros and cons of the three methods concerning profile control, surface roughness, double sided alignment, the symmetry of the nozzle and the reproducibility.

\section{- Deep reactive ion etching}

Our research shows that the DRIE fabrication method, when a negative taper is considered, is not suitable for making a conical nozzle. Although the method is very suitable for making smooth sidewalls especially when cryogenic etching with $\mathrm{SF}_{6} / \mathrm{O}_{2}$ plasma is considered, the profile could not be controlled. Two approaches have been explored to obtain the desired half angle of the nozzle; namely, the ion angular distribution (IAD) and image force (IF) effect. The IAD is broadened under certain process settings causing the ions to travel towards the wafer under an angle. The largest angle that could be obtained is about $5^{\circ}$ which is insufficient. Due to the IF effect, ions are deflected towards the sidewall of an etched feature. Thereby the sidewall is etched and a negative taper can be obtained. The process could not be tuned in such a way that it results in a proper negative taper suitable for the nozzle. By these two effects no satisfactory negative taper could be obtained and therefore this method is discarded. Despite of this some remarks can be made regarding the other criteria. From Figure 4-4 one can see that the throat of the nozzle has a gradual changing curvature, no sharp edges are present. This is a nice feature of the combined isotropic and directional etch step and would be very interesting for a nozzle throat. In general, the resulting surface roughness of this method is in the submicron range which is quite good. Furthermore the symmetry and reproducibility of this fabrication method are excellent.

\section{- Femtosecond laser macbing}

FLM is a good method to fabricate nozzles in silicon especially for rapid prototyping. The half angle of the converging and diverging part of the nozzle can be tuned according to the requirements. With this method the radius of curvature of the throat is not controlled, it is a sharp corner which has a negative effect on the performance. The roughness estimated from SEM images of the sidewall is in 
the order of microns. The double sided alignment is a critical step in this method, since little misalignment results in an asymmetrical nozzle shape. It is shown how the nozzle throat area is influenced by the error in double sided alignment. The differences in individual nozzle throat area are within 10\% which is quite a large range. Therefore this method suffers from poor reproducibility due to the error in alignment.

\section{- Powder blasting and heat treatment}

The method of powder blasting in combination with heat treatment results in a very smooth nozzle sidewall which is especially important in the throat. This technique improves surface quality, settles the profile angle and removes the need for double side alignment. The radius of curvature and the position of the throat are controlled by the temperature step and can be adjusted according to specifications.

A disadvantage of this method is the short inlet length, but the impact on the performance is minor. Furthermore, the powder blasting process is not very accurate and introduces deviations in the dimensions of the nozzle. Care must be taken with the alignment of the abrasive beam during the powder blasting process. The beam of $\mathrm{Al}_{2} \mathrm{O}_{3}$ particles should be exactly perpendicular to the wafer surface to obtain a symmetrical hole.

\begin{tabular}{|l|l|l|l|l|l|l|l|l|l|l|}
\hline $\begin{array}{l}\text { Fabrication } \\
\text { method }\end{array}$ & \multicolumn{3}{l|}{ Profile control } & \multicolumn{3}{l|}{ Roughness } & Alignment & Symmetry & $\begin{array}{l}\text { Reproduci } \\
\text { bility }\end{array}$ \\
\hline & Inlet & Outlet & Throat & Inlet & Outlet & Throat & & & \\
\hline \hline FLM & + & + & - & - & - & - & - & - & - \\
\hline RIE & - & - & + & + & + & + & $\mathrm{n} / \mathrm{a}$ & + & + \\
\hline $\begin{array}{l}\text { Powder blasting } \\
\text { Heat treatment }\end{array}$ & - & + & + & + & + & + & $\mathrm{n} / \mathrm{a}$ & $+/-$ & $+/-$ \\
\hline
\end{tabular}

Table 4-I: pros and cons of the different fabrication methods. 
It can be concluded that the nozzles manufactured by FLM and by powder blasting in combination with heat treatment are with great potential. Since the radius of curvature of the nozzle throat and the sidewall roughness are the most important parameters for the nozzle performance the latter method is preferred at this moment. The FLM method requires further improvements which might lead to controllable curvatures, and a larger freedom of choice for the diverging angles and a smaller roughness.

\subsection{References}

[1] Moerel J. L. P. A., Sanders H. M., Louwerse M. C., et al., Development of micro propulsion system technologies for minisatellites in the Netherlands, 5th International spacecraft propulsion conference (2008).

[2] Bayt R., Kenneth S., Breuer A. and Arturo A., DRIE-fabricated nozzles for generating supersonic flows in micropropulsion systems, Proceedings of the Solid-State Sensor and Actuator Workshop, Hilton Head, SC (1998).

[3] Sutton G. P. and Biblarz O., Rocket Propulsion Elements: an introduction to the engineering of rockets, 7th edition, John Wiley \& Sons, inc., New York (2001).

[4] Jansen H.V., de Boer M.J., Unnikrishnan S., Louwerse M.C. and Elwenspoek M.C., Black silicon method X: a review on high speed and selective plasma etching of silicon with profile control, J. Micromech. Microeng. 19 (2009).

[5] Tsujimoto K., Tachi S., Ninomiya K., Suzuki K., Okudaira S. and Nishimatsu S., New side wall protection technique in microwave plasma etching using a chopping method, 18th Conference on Solid State Devices and Materials, Tokyo (1986), p. 229.

[6] Jansen H.V., de Boer M.J., Wiegerink R., et al., Black silicon method VII: RIE lag in high aspect ratio trench etching of silicon, Microelectronic Engineering 35 (1997), p. 45.

[7] Waits C. M., Morgan B., Kastantin M. and Ghodssi R., Microfabrication of 3D silicon MEMS structures using gray-scale lithography and deep reactive ion etching, Sensors Actuators A 119 (2005), p. 245.

[8] Groenendijk M. N. W. and Meijer J., Microstructuring using femtosecond pulsed laser ablation, J. Laser Appl. 18 (2006), p. 227. 
[9] Chichkov B N, Momma C., Nolte S., von Alvensleben F. and Tünnermann A., Femtosecond, picosecond and nanosecond laser ablation of solids, Appl. Phys. A 63 (1996), p. 109.

[10] Zandbergen B.T.C., Louwerse M.C., Groenendijk M.N.W., et al., Initial Development of a Miniature Cold Gas Propulsion System for Nano/MicroSatellites, 5th International Spacecraft Propulsion Conference, Crete, Greece (2008).

[11] Larson W. J. and Wertz J. R., Space Mission Analyses and Design, 2nd edition, Microcosm inc. and Kluwer Academic Publishers, Dordrecht (1992).

[12] Wensink H. and Elwenspoek M. C., Reduction of sidewall inclination and blast lag of powder blasted channels, Sensors Actuators A 102 (2002), p. 157.

[13] Wensink H., Schlautmann S., Goedbloed M. H. and Elwenspoek M. C., Fine tuning the roughness of powder blasted surfaces, J. Micromech. Microeng. 12 (2002), p. 616.

[14] de With G. and Corbijn A. J., Morphological relaxation of glass surfaces, J. Mater. Sci. 30 (1995), pp. 3155-3161. 



\section{5 \\ Valve for}

micro propulsion

This chapter deals with the design and realization of a micro valve suitable for micro propulsion. Typical for space applicable valves is the strong requirement for a very low leak rate. The problems that are encountered when leak tightness is crucial are discussed. Several MST valves that are found in literature pass the review in light of their characteristics. A novel approach is presented to make a leak tight valve by utilizing a flexible thin film membrane. Finally, the fabrication of this valve is discussed. 


\subsection{Introduction}

A valve - suitable for micro propulsion purposes - has to be very leak-tight. Leaktight seals between materials can be made with o-rings, deformable metal seals or just by solder bonds. The valve has to be extremely leak-tight in the closed state and in the open state the resistance to the gas flow has to be very low. It is this combined requirement which is hard to meet. In this chapter the ins and outs of a leak-tight MST valve are discussed. Paragraph 5.2 describes the problems that arise when low leakage is crucial, especially when miniaturization by means of MST is pursued. Several micro valves pass the review that can be found in literature. In paragraph 5.3 a new approach for making a leak-tight valve is presented. By utilizing a flexible membrane and specifically shaped valve seats low leakage is pursued. The functional design of this membrane valve is considered and the dimensions of the valve are derived. Then, in paragraph 5.4 the fabrication of the membrane valve is presented. Several technological challenges are solved that came forth during fabrication. Finally, in paragraph 5.5 the results are discussed and some conclusions are made.

\subsection{Conventional MST valves and leak-tightness}

The most important requirement for the valve is a low leak rate. Section 5.2.1 stresses on the implications of the low leakage requirement. In Section 5.2.2 we look at several micro valves that have been made in the past. Since the most important characteristic of the valve is the low leak rate the focus is mainly on valves for which the leak rate is specified.

\subsubsection{Leakage}

This section discusses the difficulty of making a valve which is sufficiently leak tight. This low leak rate on the one hand and the demand of further miniaturization on the other is shown to be contradicting. At least it is for the conventional approach.

As discussed in chapter 1, the leakage of the valve is of utmost importance for a successful mission. A high leak rate causes undesired disturbance torques and deviations in altitude, but even worse, it shortens the mission duration significantly. 
As derived in chapter 2, for a micro satellite carrying 0.8gram of nitrogen, less than 0.1 gram/year loss due to leakage is allowed. This means a valve leakage of less than $1.6 \cdot 10^{-4} \mathrm{sccm}$. In conventional valves these low leakages are obtained by using a soft layer which is pressed against a hard valve seat with a high force. Dirt particles are pressed in the soft layer to prevent leakage as is shown in Figure 5-1. The pressure that is required for soft-hard contact valves are in the order of 5 to 100 bar [1].

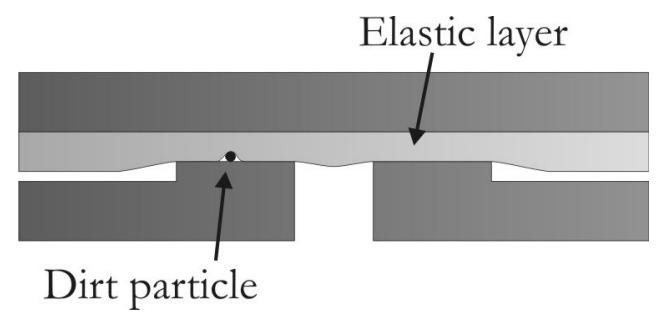

Figure 5-1: Dirt particle on the valve seat encapsulated by elastic layer.

Such a soft layer has to be elastic. The layer has to be thick enough to have enough compliance in the perpendicular direction. The most common elastic polymer or elastomer material that is used in MST is PDMS [2]. However, for our application this material is not suitable because it is permeable for gasses causing leakage. Other elastomer materials should be explored for a leak tight valve. Another disadvantage of elastomers is the fact that the elasticity is highly dependant on temperature, which is undesirable considering the temperature variations of the satellite. At lower temperatures the elasticity decreases and thus it would require more force to encapsulate a particle that is stuck in the valve.

When using an elastic layer one also needs to consider the additional stroke that is required by the actuator. The stroke that is lost is determined by the distance the elastic layer is pushed in. For piezo-actuators the stroke and force are strongly related to the size of the actuator. When pursuing miniaturization, this means that the required stroke and force should be minimized to allow for a smaller actuator. Since the stroke should be reduced as much as possible a soft layer is not the way to go. Besides this fact, the usage of a soft layer reduces the variety of fabrication techniques that can be used to manufacture the valve. An elastic layer cannot withstand the high temperatures that are required for many process steps. It is definitely not impossible but since actuator stroke is precious a soft layer is no option and is discarded. 
Another possibility is the usage of two hard surfaces that are pressed against each other with a high force to close the valve. A pressure in excess of 5000 bar is required for these kind of valves [1]. This very high pressure is needed to crush the dirt particles between the two hard surfaces and close the gap between valve seat and sealing. A disadvantage of the high pressure is that the valve seat might be damaged. Especially when a silicon valve seat is used this can cause problems. Due to the single crystalline structure of silicon, an initiated crack can easily propagate and causes large fragments of silicon to break out. This kind of valve is also not really an option because of the high force that is required and thus a larger actuator. The first generation of valves that we fabricated was one with two hard silicon surfaces. The fabrication and measurements of this valve are discussed in Appendix C. By introducing an external force to close the valve a leak-rate of $8 \cdot 10^{-4} \mathrm{sccm}$ was obtained which is 5 times worse than specified. Moreover, under this external force it became impossible to open the valve since the piezo-actuator generates insufficient force to counteract the external force. It would require a more forceful actuator, and thus a larger one, to open the valve.

The low leakage is quite a strong requirement and not easily satisfied with MST. To give an indication of the challenge this requirement brings we calculated the size of the gap between a valve seat and its sealing which would result in the maximum allowable leakage. To calculate the gap height the dimensions as indicated in Figure 5-2 are used. These dimensions come forth from the calculations made in Chapter 2. It is assumed that the flow is in the molecular range which is justified later on by calculating the Knudsen number. The mass flow through the valve is calculated by [3]:

$$
\dot{m}=\frac{4}{3 R T} \frac{\bar{v} \pi g^{2}\left(p_{1}-p_{2}\right)}{\ln \left(r_{2} / r_{1}\right)}
$$

Where $\bar{v}$ is the mean thermal velocity, $R$ is the gas constant and $T$ the temperature and $p_{1}$ and $p_{2}$ the inlet and outlet pressure, respectively. The geometry is defined by $g$ the gap height and $r_{1}$ and $r_{2}$ the inner and outer radius of the valve seat, respectively. The mass flow $\dot{m}$ is converted from $\mathrm{mol} / \mathrm{s}$ to $\mathrm{sccm}(1 \mathrm{~mol} / \mathrm{s} \approx$ $\left.1.344 \cdot 10^{6} \mathrm{scm}\right)$. At a pressure drop of $3 \mathrm{bar}-$ which is an over-estimation for the mean tank pressure - the maximum allowed leakage of $1.6 \cdot 10^{-4} \mathrm{sccm}$ is already 
reached at a gap height of $25 \mathrm{~nm}$. For this gap height the Knudsen number is 6.7 which indicates a molecular flow.

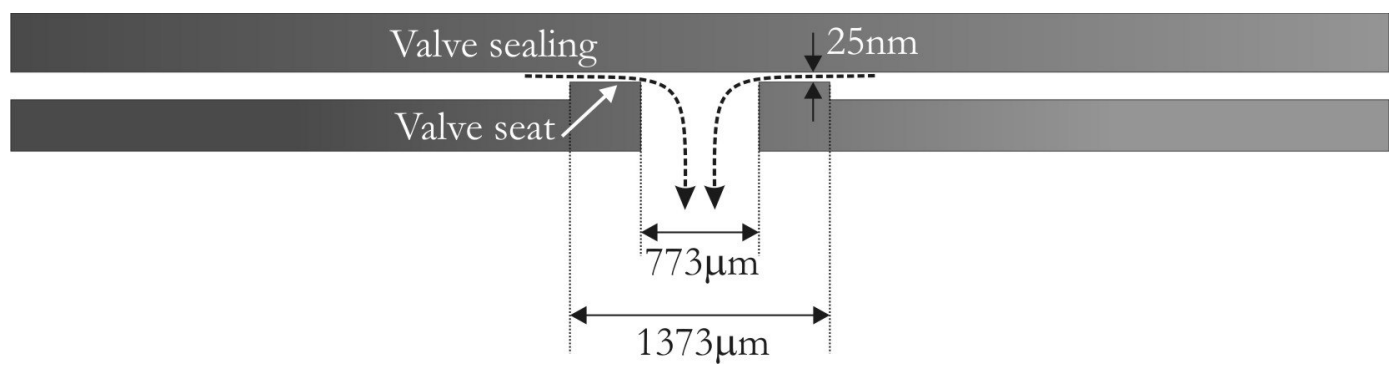

Figure 5-2: Typical geometry of a micro valve using MST.

Even if one assumes there are no particles that cause leakage it is practically impossible to obtain a surface which is that flat over a circumference in the millimeter range. When looking at the surface of a silicon wafer one finds waviness over any area of interest for which the following rule applies; the larger the area of interest the larger the wavelength and amplitude of the waves on the surface [4]. This means that a valve seat with a smaller circumference has waves with smaller amplitude, meaning less leakage than a valve with a larger circumference. Then the circumference of the valve seat should be as small as possible to minimize leakage. Unfortunately there is another limiting factor for reducing the circumference as is argued in chapter 2. The area of passage of the valve should be larger than the throat area of the nozzle to avoid choked flow in the valve. To obtain a thrust level at the upper end of the specified thrust range $(10 \mathrm{mN})$ the inner diameter of the valve has to be $773 \mu \mathrm{m}$ as calculated in chapter 2 .

We measured the waviness of a bare single side polished $525 \mu \mathrm{m}$ thick $<100>$ silicon wafer with white light interferometry. The result of a typical measurement is shown in Figure 5-3. Over a circumference of $2.5 \mathrm{~mm}$ a height difference of approximately $30 \mathrm{~nm}$ is measured. In the worse case, assuming that the valve seat and sealing are made of such silicon wafers there would be a gap of $60 \mathrm{~nm}$ between them when the valve is closed. This results in a leakage which exceeds the allowed value.

From the above discussion the following can be concluded. The conventional approach for making a leak-tight valve - with a soft-hard seal - requires quite some force and additional deflection. The hard-hard sealed valve requires an even higher force to make a tight seal. This means a large actuator which goes against the vision 
of miniaturization. Discarding the option of forceful actuators we need a smart design which neutralizes trapped particles without requiring a lot of force. The valve should also be designed in such a way that the waviness on the silicon base material, having amplitude of tens of nanometers, is not causing any leakage problems. Before presenting a new approach towards a leak-tight valve we look at some MST valves found in literature for which the leakage is specified.
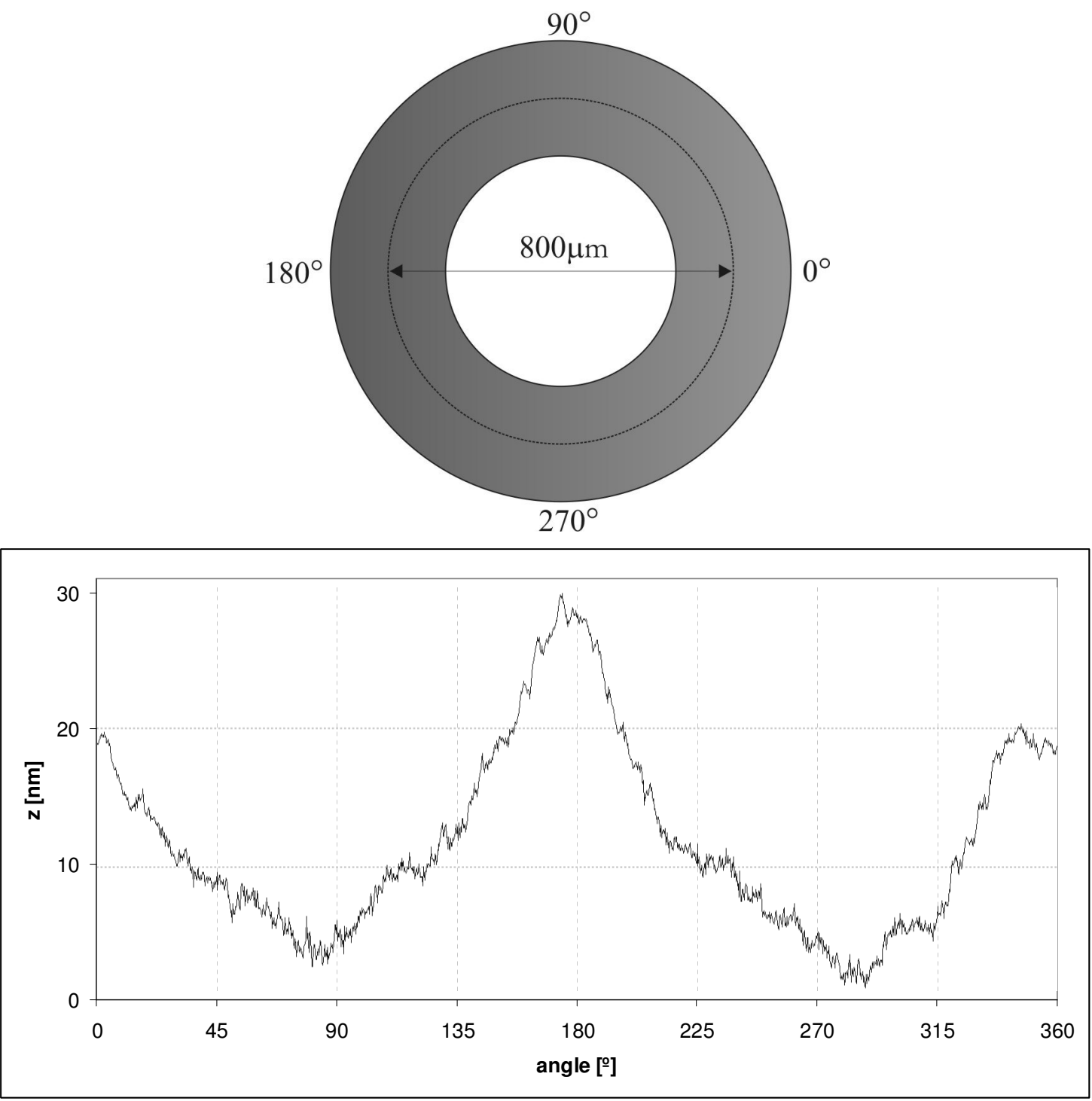

Figure 5-3: Waviness on a single side polished $<100>$ silicon wafer, having a diameter of $100 \mathrm{~m}$ and a thickness of $525 \mu \mathrm{m}$. Measured is done on the polished side with a white light interferometer. 


\subsubsection{Micro machined valves, state-of-the-art}

In this section several micro valves pass the review and are compared for the following characteristics; leak rate, power consumption and response time. Many micro valves have been fabricated over the last decades. Every one of them designed for particular applications. For an overview of developed micro valves one can look into the following references $[5,6]$. Although a lot of valves have been developed, not many focus on leak-tightness for gasses. In our case less than 0.1 gram/year loss of nitrogen due to leakage is allowed which means a valve leakage of less than $1.6 \cdot 10^{-4} \mathrm{sccm}$. When focusing on active gas valves, which have been tested for leakage, a few can be found, all of them being hard-hard valves. As an example, only one soft-hard valve is discussed, although no leak rate is specified. In Table 5-I the characteristic properties of the valves are summarized. Beware that it is difficult to give a good comparison between the various characteristics. For example, the power is not specified for every valve, as some only specify voltage or current. The different valves are discussed shortly.

Choi et al presented a normally closed electro-magnetic actuated valve [7]. A schematic of the valve is shown in Figure 5-4.a. An electro-magnet acts on a thin $\mathrm{NiFe}$ film to open the valve. This permalloy is deposited on a silicon membrane which seals the valve seat. In the open state, a flow rate of $0.02 \mathrm{sccm}$ is obtained at a pressure of $9.7 \mathrm{kPa}$ and a current through the electro-magnet of $550 \mathrm{~mA}$. The measured leak rate is $1.5 \cdot 10^{-2} \mathrm{sccm}$ at a pressure of $4.8 \mathrm{kPa}$.

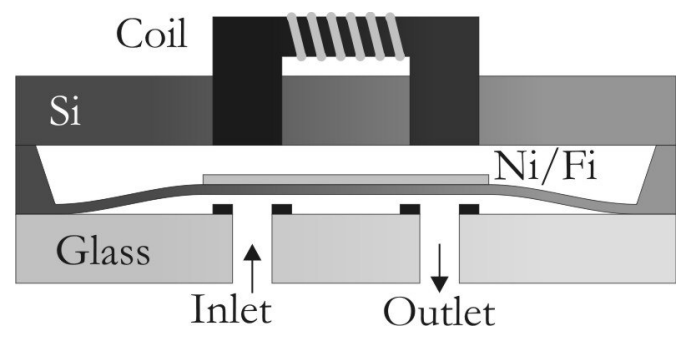

a)

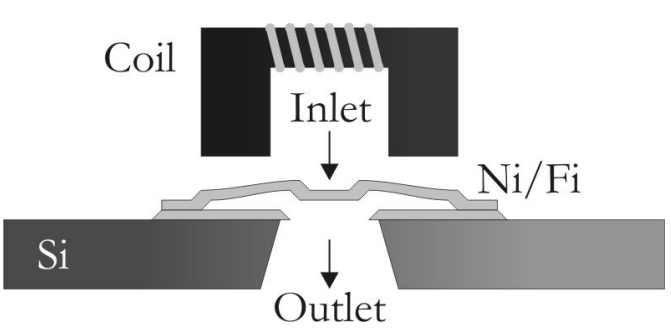

b)

Figure 5-4: a) Choi b) Hirano 
Hirano et al used the same actuation principle for a normally closed valve [8]. Figure 5-1.b shows the schematic. The valve is fabricated in such a way so that very tight seal is ensured. Any waviness on the valve seat are copied in the $\mathrm{NiFe}$ sealing. When a voltage is applied to an external coil the NiFe sealing is pulled upwards and the valve opens. At $25 \mathrm{~V}$ and a pressure of 1 bar a flow rate of $0.62 \mathrm{sccm}$ is measured. The measured leak rate is $1.1 \cdot 10^{-2} \mathrm{sccm}$ at a pressure of $100 \mathrm{kPa}$.

As part of the MIT micro-engine project a normally closed on/off valve has been presented by Yang X et al [9]. The valve is depicted in Figure 5-5.a. It is electrostatically actuated by applying a voltage over a top electrode and the boss electrode or valve sealing. The flow rate through the valve is $45 \mathrm{sccm}$ when $136 \mathrm{~V}$ is applied at a pressure of $9 \mathrm{bar}$. The resistive power that is consumed is less than $0.04 \mathrm{~mW}$ and the response time of this valve is $20 \mathrm{~ms}$. The measured leak rate is $2.1 \cdot 10^{-3} \mathrm{sccm}$ at a pressure of $170 \mathrm{kPa}$.

A normally closed bimetallic diaphragm valve is manufactured by Jerman [10]. It is shown in Figure 5-5.b. A Teflon ${ }^{\text {TM }}$ coated Kapton ${ }^{\text {TM }}$ diaphragm seals a silicon valve seat. At an applied power of $350 \mathrm{~mW}$ and a pressure of $138 \mathrm{kPa}$ a flow rate of $90 \mathrm{sccm}$ is measured. The response time of this valve is larger than $100 \mathrm{~ms}$. Opening of the valve can be accelerated by applying more power but the closing time is limiting by the cooling of the valve. The measured leak rate is $5.7 \mathrm{sccm}$ at a pressure of $35 \mathrm{kPa}$.

Rich et al presented a normally open thermo-pneumatic valve [11]. Figure 5-5.c shows the valve schematic. A cavity contains a volatile fluid which vaporizes when heat is applied. Under the vapor pressure a corrugated silicon membrane is deflected and closes the valve. With an input pressure of $133 \mathrm{kPa}$, the valve closes when $350 \mathrm{~mW}$ of power is applied. After closing, $30 \mathrm{~mW}$ of power is enough to keep it shut. In the open state a flow of $400 \mathrm{sccm}$ is measured with a pressure of $200 \mathrm{kPa}$. The measured leak rate is $1 \cdot 10^{-3} \mathrm{sccm}$ at a pressure of $200 \mathrm{kPa}$. A thermodynamic model suggests a response time of 1 s for this valve. 


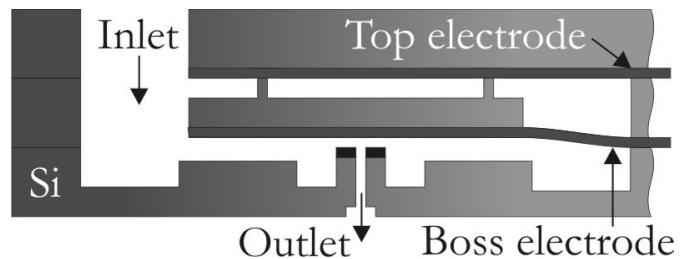

a)

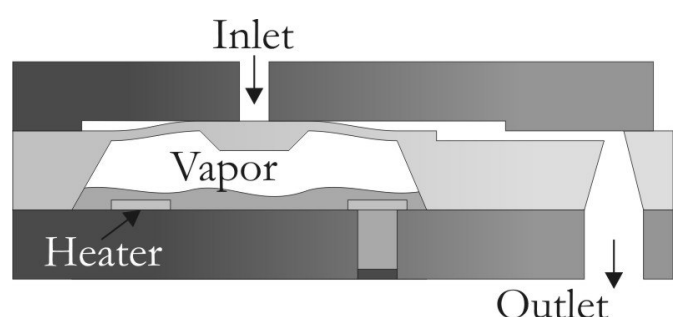

c)

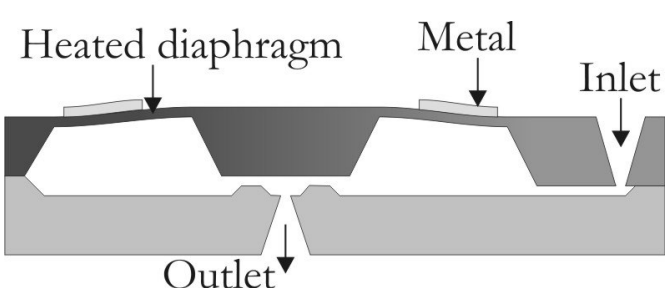

b)

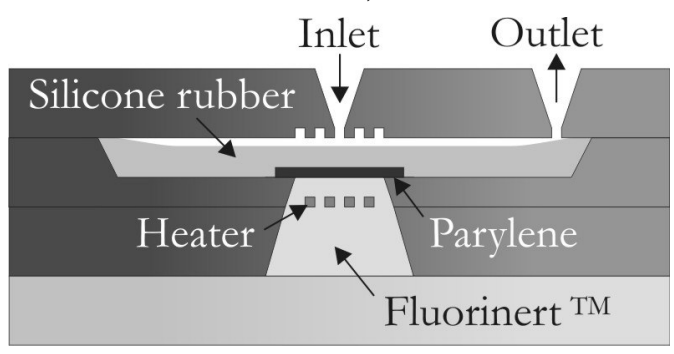

d)

Figure 5-5: a) Yang $X$ b) Jerman c) Rich d) Yang

Yang et al have presented a thermo-pneumatically actuated, normally open, silicone rubber membrane valve [12]. The valve is depicted in Figure 5-5.d. The heaters are suspended inside a fluid with good thermal properties. The fluid is separated from the silicon rubber by a Parylene layer. Parylene acts as a barrier to avoid absorption of moisture in the rubber. The silicone rubber is used to seal the valve seats. It is hypothesize that particles are encapsulated by the rubber membrane due to its elastic properties and thereby avoiding leakage. Unfortunately no value for gas leakage is given. As stated earlier, the problem with rubber is that it is permeable for gasses, limiting the use of this material to applications where leakage is not critical [13]. In the open state a flow rate of $8790 \mathrm{sccm}$ is measured with a pressure of $345 \mathrm{kPa}$. When a power of $35.5 \mathrm{~mW}$ and a pressure of $228 \mathrm{kPa}$ are applied the valve is closed. The response time of the valve is in the order of tens of seconds.

Rogge et al fabricated a polymer normally closed micro-valve [14]. The valve is actuated by a piezo bending disk as shown in Figure 5-6.a. To increase the deflection of the polyimide membrane that closes the valve a hydraulic mechanism is designed. With a pressure of $100 \mathrm{kPa}$ and an applied voltage of $140 \mathrm{~V}$ a flow rate of $430 \mathrm{sccm}$ is obtained. The measured leak rate is $3.3 \mathrm{sccm}$ at a pressure of $200 \mathrm{kPa}$. The response time of the valve is $1.8 \mathrm{~ms}$. Although the valve is a normally closed it requires power to keep it closed at higher pressures. 
As part of a cold gas thruster system a piezo-electric actuated micro valve is presented by Köhler [15]. No schematic of the valve is available. The valve is piezo electrically actuated. When $50 \mathrm{~V}$ is applied to the piezo elements, a maximum stroke of $4 \mu \mathrm{m}$ obtained. The valve seats have been covered with either Parylene or Titanium-Aluminium to avoid degradation of the valve seat. The measured leak rate is $2.1 \cdot 10^{-4} \mathrm{sccm}$.

Another piezo-electric actuated valve is developed by Yang E-H et al [16]. The schematic of the valve is shown in Figure 5-6.b. A custom designed piezo-electric stack actuator capable of delivering a blocking force of $1000 \mathrm{~N}$ or a deflection of $5 \mu \mathrm{m}$ when $60 \mathrm{~V}$ is applied. This valve is designed to work at extreme high pressure. At a pressure of $689 \mathrm{kPa}$ and $20 \mathrm{~V}$ a flow rate of $80 \mathrm{sccm}$ is measured. The measured leak rate is $5.2 \cdot 10^{-6} \mathrm{sccm}$ at a pressure of $690 \mathrm{kPa}$. The response time of the valve is approximately $30 \mu \mathrm{s}$.
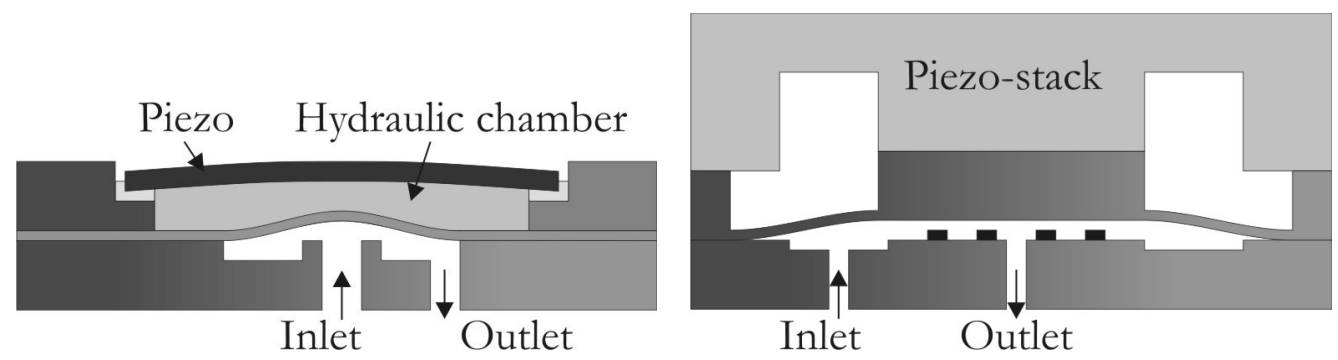

Figure 5-6: a) Rogge b) Yang E-H

The power consumption and response time of these valves are considered first, before the leakage is addressed. From Table 5-I one can see that the lowest specified power is achieved by the electro-statically actuated valve of Yang X. et al. They specified the resistive power consumption of the valve which is $0.04 \mathrm{~mW}$. It must be noted that this is not a very useful figure because DC-DC conversion is not taken into account. The piezo-electric actuated valve of Yang E-H et al. then has the lowest power consumption. The thermal actuated valves require the most power and they also have the largest response time. The response time of the piezo electrically actuated valves are superior.

The measured leakages of the valves are shown in Table 5-I. Most references specify the leak rate in standard cubic centimeter per minute $(\mathrm{sccm})$. When the leakage is given in other units it is converted to sccm. The leak rate measurements presented in the references are done under various differential pressures and with 
various gasses. In case a Helium leakage was specified it is converted to a Nitrogen leakage by assuming that the leak rate is inversely proportional to the square root of the molecular mass [17]. Furthermore, to be able to compare the measured values, we calculate the leakage that would occur with a tank pressure of $3 \mathrm{bar}(300 \mathrm{kPa})$, which is an over-estimation of our mean tank pressure. Therefore, we assume a linear relation between the leak rate and applied differential pressure [9]. In general this assumption is not valid because it depends on the design of the valve. Consider for example the valves of Choi, Rich, Xing and Rogge (Figure 5-4.a, Figure 5-5.c, Figure 5-5.d and Figure 5-6.a). All these valves use a membrane which closes the valve. The high pressure side of these valves - i.e. the inlet - is designed in such a way that it pushes the valve open. This means the higher the inlet pressure the more the membrane will be lifted off from the valve seat and thus a larger leak hole. For the other valves the pressure is working in favour because it is helping to close valve. For the valve of Jerman (Figure 5-5.b) it is not clear whether the inlet pressure is also present above the embossed diaphragm. If so it would help to close the valve. For simplicity it is assumed that the leakage has a linear relation with pressure. Most valves that are considered have a leakage which is much too high. They do not come near to the maximum allowed leakage of $1.6 \cdot 10^{-4} \mathrm{sccm}$. The valve presented by Köhler is close, with a leakage of $6.4 \cdot 10^{-4} \mathrm{sccm}$. The valve with the best leak performance is the piezo-electric actuated valve of Yang E-H [16]. A leak-rate of $5.2 \cdot 10^{-6} \mathrm{sccm}$ is measured at a pressure of $689 \mathrm{kPa}$. This corresponds to a leakage of $2.3 \cdot 10^{-6} \mathrm{sccm}$ at $300 \mathrm{kPa}$, which is 70 times less than the $1.6 \cdot 10^{-4} \mathrm{sccm}$ leakage which is required for our system. This low leakage is achieved as a result of the extreme high closing pressure of $954 \mathrm{MPa}$. A specifically designed piezo-actuator stack is attached to the valve in such a way that it is under tension. When actuated the boss is lifted $5 \mu \mathrm{m}$ upwards and the valve is opened. This actuator is not extremely big - it measures: $8.4 \mathrm{~mm} \cdot 5 \mathrm{~mm} \cdot 6 \mathrm{~mm}$ - but it is still the biggest part of the valve.

Although the leak performance of this valve is superior we chose a different approach than the brute force method. This choice is driven by two reasons. First, to allow for further miniaturization a smaller actuator is preferred and thus less force. Second, the high closing force might damage the valve seat as discussed in the previous section. In the next paragraph a novel approach to a leak-tight valve is presented. 


\begin{tabular}{|c|c|c|c|c|c|c|c|c|c|}
\hline \multirow[b]{2}{*}{ Reference } & \multirow[b]{2}{*}{ Type } & \multirow[b]{2}{*}{ Gas } & \multirow[b]{2}{*}{$\begin{array}{l}\text { Power/ } \\
\text { Voltage/ } \\
\text { Current }\end{array}$} & \multicolumn{3}{|c|}{ Leakage } & \multicolumn{2}{|c|}{ Flow rate } & \multirow[b]{2}{*}{$\begin{array}{l}\text { Response } \\
\text { (msec) }\end{array}$} \\
\hline & & & & $\begin{array}{l}\text { Applied } \\
\text { pressure } \\
(k P a)\end{array}$ & $\begin{array}{l}\text { Measured } \\
\text { leakage } \\
\left(\operatorname{sccm} N_{2}\right)\end{array}$ & $\begin{array}{l}\text { Leakage at } \\
300 \mathrm{kPa} \\
\left(\operatorname{sccm} \mathrm{N}_{2}\right)\end{array}$ & $\begin{array}{l}\text { Applied } \\
\text { pressure } \\
(\mathrm{kPa})\end{array}$ & $\begin{array}{l}\text { Measured } \\
\text { flow } \\
\text { (sccm) }\end{array}$ & \\
\hline Choi [7] & NC/EM & & $550 \mathrm{~mA}$ & 4.8 & $1.5 \cdot 10^{-2}$ & $9.2 \cdot 10^{-1}$ & 9.7 & 0.02 & \\
\hline Hirano [8] & $\mathrm{NC} / \mathrm{EM}$ & $\mathrm{He}$ & $25 \mathrm{~V}$ & 100 & $1.1 \cdot 10^{-2}$ & $3.4 \cdot 10^{-2}$ & 100 & 0.68 & \\
\hline Yang X [9] & NC/ES & $\mathrm{He}$ & $0.04 \mathrm{~mW} / 136 \mathrm{~V}$ & 170 & $2.1 \cdot 10^{-3}$ & $3.8 \cdot 10^{-3}$ & 900 & 45 & 20 \\
\hline Jerman [10] & $\mathrm{NC} / \mathrm{BM}$ & $\mathrm{N}_{2}$ & $350 \mathrm{~mW}$ & 34.5 & 5.7 & 49 & 138 & 90 & $>100$ \\
\hline Rich [11] & $\mathrm{NO} / \mathrm{TP}$ & & $30 \mathrm{~mW}$ & 200 & $1.0 \cdot 10^{-3}$ & $1.5 \cdot 10^{-3}$ & 200 & 400 & 1 \\
\hline Yang [12] & NO/TP & $\mathrm{N}_{2}$ & $35.5 \mathrm{~mW}$ & 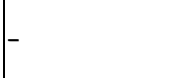 & - & - & 345 & 8790 & $>20000$ \\
\hline Rogge [14] & $\mathrm{NC} / \mathrm{PE}$ & $\mathrm{N}_{2}$ & $140 \mathrm{~V}$ & 200 & 3.3 & 4.9 & 50 & 430 & 1.8 \\
\hline Köhler [15] & $\mathrm{NC} / \mathrm{PE}$ & $\mathrm{He}$ & $50 \mathrm{~V}$ & - & $2.1 \cdot 10^{-4}$ & $6.4 \cdot 10^{-4}$ & & & \\
\hline Yang E-H [16] & $\mathrm{NC} / \mathrm{PE}$ & $\mathrm{He}$ & $20 \mathrm{~V} / 0.3 \mathrm{~mW}$ & 690 & $5.1 \cdot 10^{-6}$ & $2.3 \cdot 10^{-6}$ & 689 & 80 & 0.03 \\
\hline Requirements & & $\mathrm{N}_{2}$ & $<100 \mathrm{~mW}$ & & & $<1.6 \cdot 10^{-4}$ & & & $<1$ \\
\hline
\end{tabular}

Table 5-I: Characteristic of several micro valves (NO Normally Open, NC Normally Closed, EM electro-magnetic, ES electro-static, BM bimetallic, TP thermo-pneumatic, PE piezo-electric) 


\subsection{The flexible membrane valve}

In chapter 2 the top level requirements are described for a valve which can be used in a micro propulsion system. These requirements form the basis for the detailed design presented in this section. First the functional design is presented in section 5.3.1. In section 5.3.2 the dimensions of the valve are derived.

\subsubsection{Functional valve design}

This section discusses the functional design of the valve. First an overview is given of the valve structure before focusing on the sealing mechanism. The novelty of this valve lies in the sealing mechanism. It ensures a leak tight valve without requiring a high force to close the valve. Furthermore, no additional stroke is required which is the case for a soft-hard sealed valve.

Since the silicon is not perfectly flat, two surfaces that are not deformable will not make a perfect seal when they meet. To solve this problem a valve sealing is needed that is free to settle towards the valve seat. We propose to use a deflectable thin film membrane which is pulled over several concentric valve seats. Figure 5-7 shows a cross sectional view of the membrane valve. The valve is made of two silicon wafers. The top wafer contains a thin film membrane which is pulled over the valve seats of the bottom wafer. To be able to lift the membrane from the valve seats a piezo-disc actuator is used and to allow for this upward motion a spiral spring mechanism is shaped in the top wafer. The two wafers are processed separately and at last bonded together. During this bonding step the spiral spring is put under tension, it is bending a little bit upwards. The spring is exerting a force on the membrane and thereby the membrane is pulled over the valve seats. When focusing underneath the spiral spring in Figure 5-7 another membrane is visible. This thin film membrane is perforated with $2 \mu \mathrm{m}$ holes to function as a filter. Particles larger than $2 \mu \mathrm{m}$ are inhibited to pass through the filter. The integration of a filter in the valve has a big advantage. The valve is fabricated in a Cleanroom environment meaning it is clean when the fabrication is ready. With the filter integrated, the valve is protected - a priori - against particles when exposed to a dirtier environment for testing. 


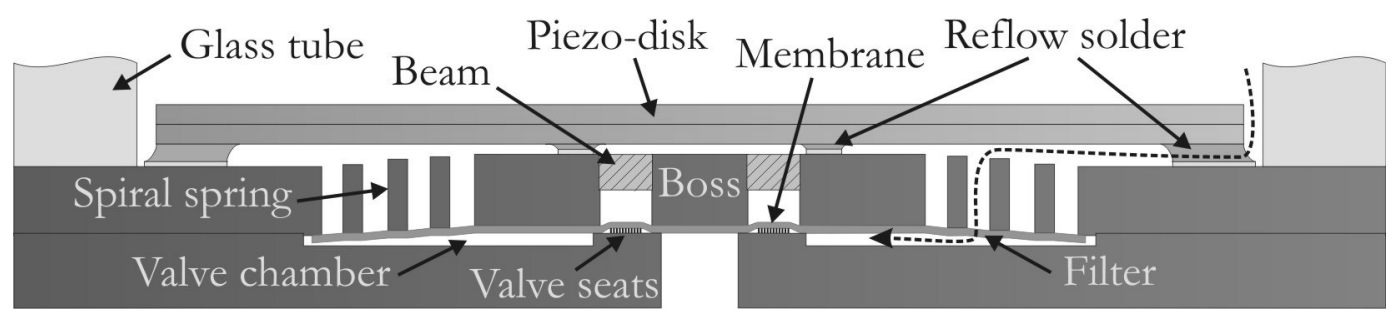

Figure 5-7: Membrane valve with glass tube and piezo-disk actuator.

A top view of the top wafer is shown in Figure 5-8.a. The area were the glass tube is bonded to the silicon valve is indicated by the hatched pattern. The piezo-disc is attached to the valve by reflow soldering. The diagonal patterned areas indicate the points where the piezo-disc is attached. The outer solder pads are interrupted to allow the gas to flow around the piezo disc as indicated in Figure 5-7 by the dashed line. In Figure 5-8.b a close-up of the middle part of the valve is shown. Above the outlet the membrane is strengthened by a silicon boss to avoid rupture of the thin membrane due to the tank pressure on one side of the membrane and vacuum on the other side. The silicon boss is connected to the outer part by beams. This is the top surface of the silicon wafer. When focusing in the depth - beneath the top surface - the membrane is visible as the light gray part. It is positioned on the backside of the wafer.

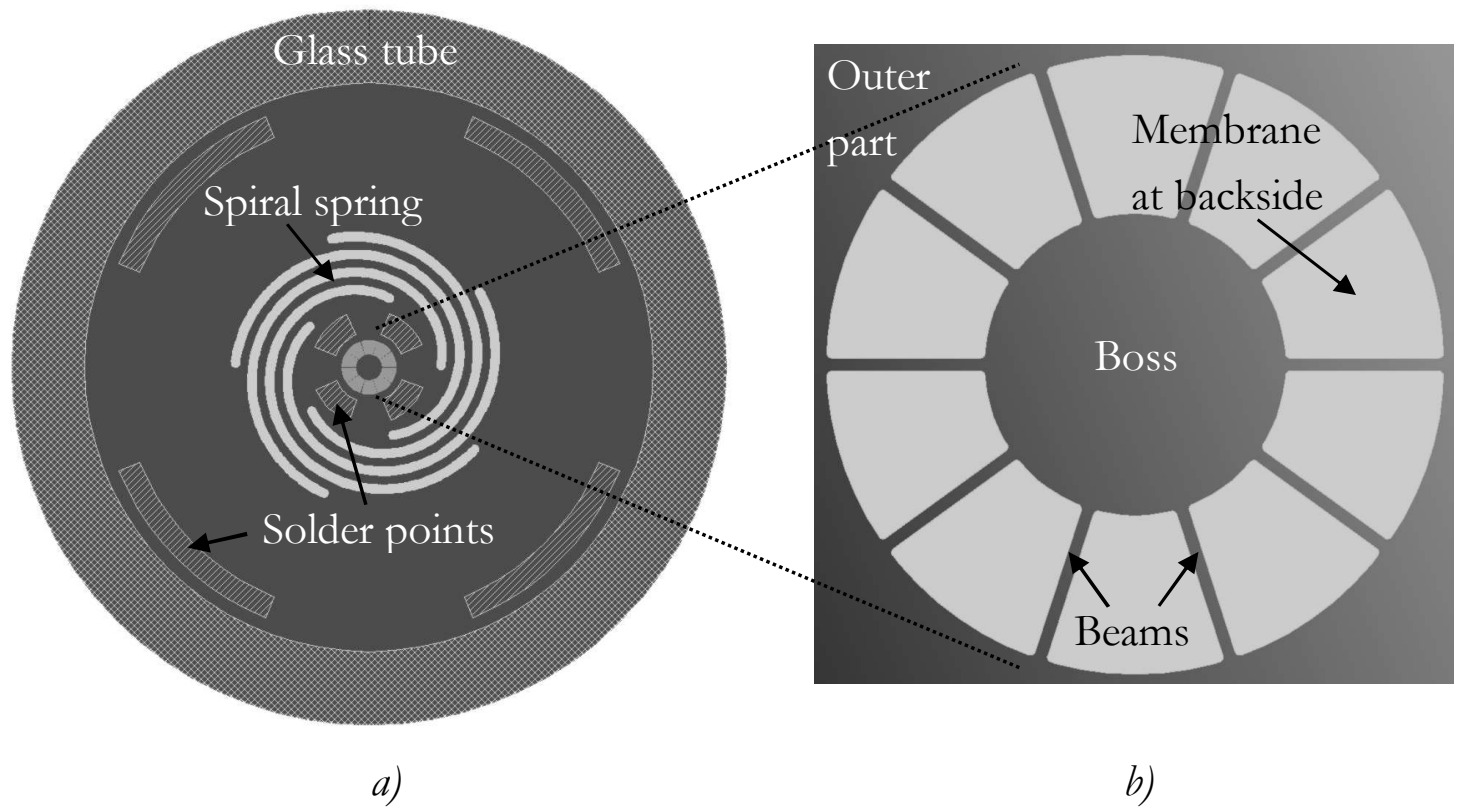

Figure 5-8: a) Top view of the membrane wafer b) Zoom in on the middle part of the valve 
In Figure 5-9 a close up of the cross sectional view of the middle part of the valve is shown. In the top picture the valve is closed and the membrane is pulled over the valve seats. The spiral spring mechanism is forcing the membrane to bend over the valve seats. This passive force is indicated by $F_{s}$ and ensures a normally closed valve and thus no power is required in the closed state.

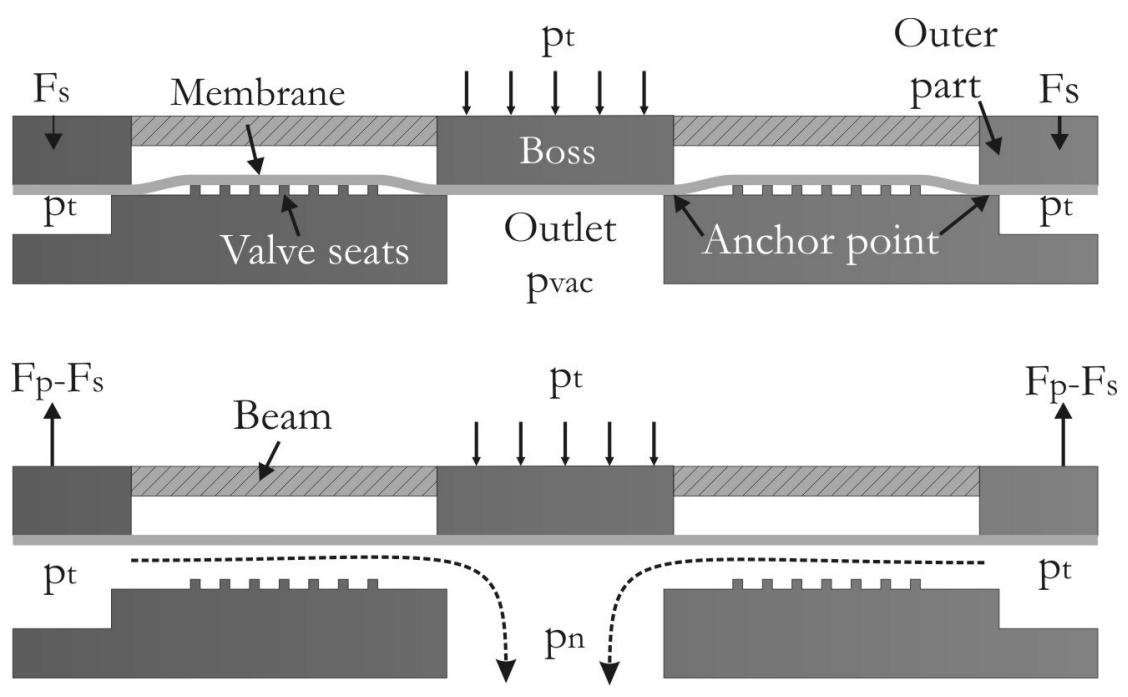

Figure 5-9: Thin film membrane valve. Top: closed valve. Bottom: open valve.

In the open state the piezo-actuator is used to pull the membrane upwards allowing a gas flow through the valve. The force which the piezo-disc is generating is indicated by $F_{p}$. The spiral spring should be designed in such a way that it requires a maximum force of $0.3 \mathrm{~N}$ to pull it at least $16.8 \mu \mathrm{m}$ upwards. The piezo-actuator can deliver $0.3 \mathrm{~N}$ force at this deflection. The silicon boss is connected to the outer part - there where the piezo-actuator acts on - by the beams as indicated in Figure 5-8 and Figure 5-9. If this would not be the case the valve would not open due to the tank pressure which is pushing the silicon boss onto the valve outlet. The membrane might rupture when pulling only the outer part of the valve upwards.

At the moment the valve is opened the valve acts as a series resistance for the gas flow with the nozzle. The nozzle is positioned directly behind the valve outlet to minimize the volume between them. This volume acts as a hydraulic capacitance for which the following applies; the larger the dead volume the longer it takes before a steady pressure $\left(p_{n}\right)$ is reached in front of the nozzle. For very precise positioning of satellites it is desired to reduce this volume to minimize inaccuracies in thrust levels. 
To give an approximation of the time it takes, to reach a steady pressure in front of the nozzle, we used 20-sim to simulate the behavior of this dynamic system [18]. The pressure in front of the nozzle is calculated by:

$$
p_{n}=\frac{R T}{V} \int\left(\dot{m}_{v}-\dot{m}_{n}\right) \cdot d t
$$

Where $\dot{m}_{v}$ and $\dot{m}_{n}$ are the mass flow through the valve and the nozzle, respectively. The formulas for the mass flows are given in chapter 2. Furthermore, $R$ is the gas constant, $T$ the temperature and $V$ the volume in between the valve and the nozzle which measures is $1.1 \cdot 10^{-10} \mathrm{~m}^{3}$. For this small volume we found that it will take approximately $20 \mu$ s to reach a steady pressure in front of the nozzle. This is well within the required response time of $1 \mathrm{~ms}$. An indication for the pressure drop over the valve has been calculated in chapter 2 .

In Figure 5-10.a a top view of the bottom wafer is given, showing the outlet and the valve chamber also indicated in Figure 5-7. Six anchor points are shown in Figure 5-10.b, which are also indicated in Figure 5-9. The silicon boss of the top wafer rests on the inner three anchor points while the outer part is supported by the outer anchor points.

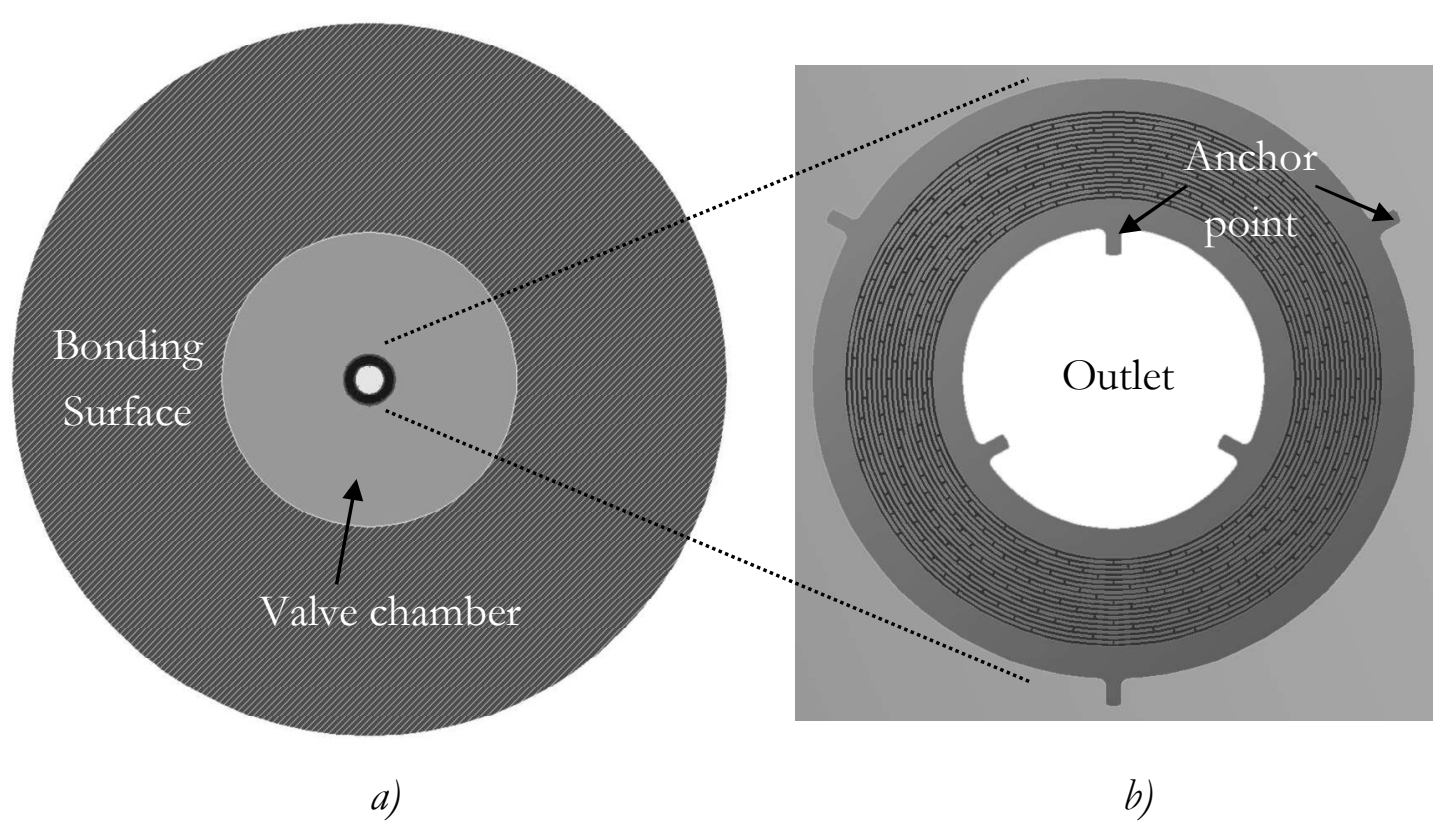

Figure 5-10: a) Top view of the valve seat wafer. b) Zoom in on the valve seats, also showing 6 anchor points 
The anchor points should be small in area to reduce the chance that particles get stuck in this part of the valve. When this happens the membrane is not pulled over the valve seat resulting in leakage as is depicted in Figure 5-11. This is a weak point in the design of the valve, but we can design in such a way that it is not favorable for a particle to stay in this position as explained later on. If these anchor points were to be left out the boss is pulled into the outlet due to the pressure difference. The membrane is then stretched too much and might break as is shown in Figure 5-12.

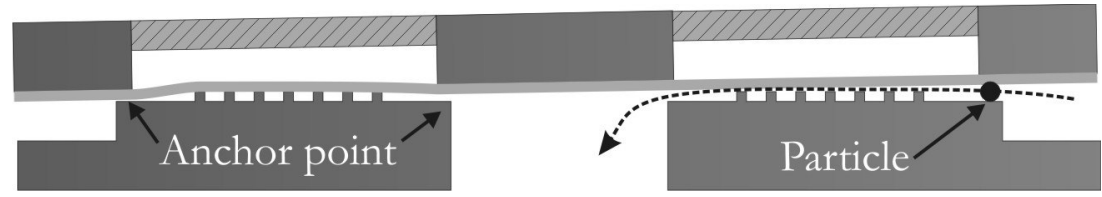

Figure 5-11: Leakage caused by a particle which is stuck at an anchor point

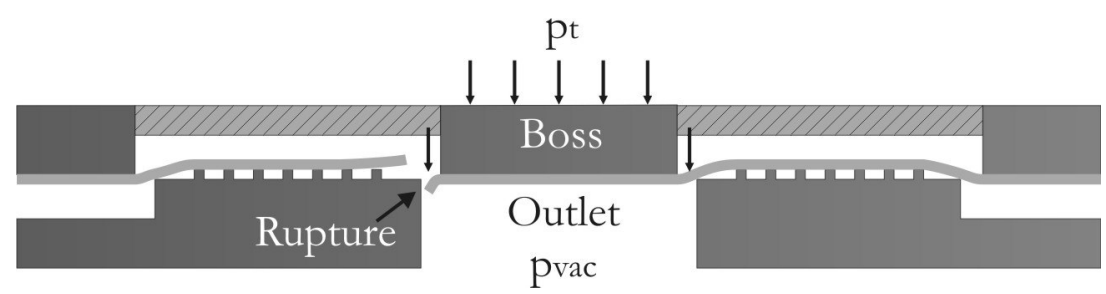

Figure 5-12: The Anchor points are left out resulting in possible rupture of the membrane

With the integration of a $2 \mu \mathrm{m}$ filter in the valve large particles are inhibited to enter the valve. However, particles smaller than $2 \mu \mathrm{m}$ can still cause significant leakage. The membrane and valve seats have to be designed in such a way that these particles will be encapsulated. This is a similar approach as the silicone rubber membrane valve of Yang et al [12]. The difference is that we use a membrane which is not permeable for gasses and since the membrane is not elastic it needs fewer stroke. Figure 5-13 shows how a particle is trapped under the membrane. In the worst case such a particle is positioned on a valve seat, rather than in between them. The membrane has to be flexible enough to bend around it. The pressure under the membrane is lower than above it when the valve is well closed. This pressure difference over the membrane is actually helping to deform the membrane around the particle. It is like a rubber balloon which is pulled over a tube forming a membrane. While the tube is depressurized the balloon is pulled inside the tube. The better the balloon is able to seal the top of the tube the higher the pressure 
difference over the membrane and thus more deformation of the membrane. When there is leakage the pressure difference is lower and the balloon is pulled in with less force. One initially needs a rather good sealing before the pressure is working in favor. This raises the question what will happen when the tank pressure is decreasing. The pressure difference over the membrane reduces and the membrane will bend less resulting in a larger opening due to particles. For a simple hole with a fixed size the amount of leak has a linear relationship with pressure. In the case of our membrane valve this relation might not be linear. However, the lower limit of the operation pressure is 1 bar and thus - below this value - the valve leakage is not critical. Only in the final stage of the mission, when the propellant tank is almost empty, the leakage might increase considerably.

To be able to neutralize particles with a size up to $2 \mu \mathrm{m}$, the cavities in between the valve seats should be $2 \mu \mathrm{m}$ deep as indicated by $h$ in Figure 5-13. Particles that are then trapped in between the valve seats do not cause any bending of the membrane. The height difference between the anchor points and the valve seats should be larger than the waviness of the wafer. This height difference is indicated by $d$.

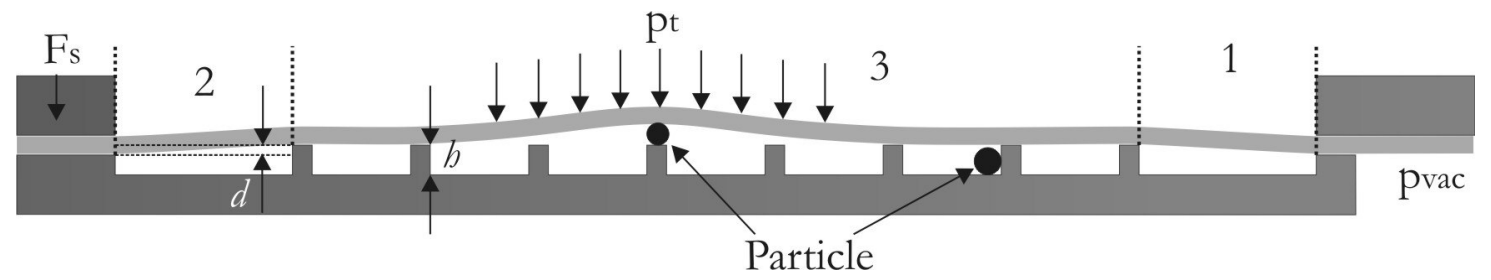

Figure 5-13: Cross sectional view: encapsulated particles under the membrane.

Figure 5-14.a shows a top view of two encapsulated particles trapped on top of the valve seats. The larger the particle the larger the membrane is deflected causing a larger open area as indicated by the circles around the particles. The valve seats are divided into compartments to reduce the chance that several particles together are causing leakage. In Figure 5-14.a the shaded compartments are connected due to the trapped particles. When the valve seats are not divided into compartments two particles - which are positioned much farther apart - can already induce leakage as is shown in Figure 5-14.b. 


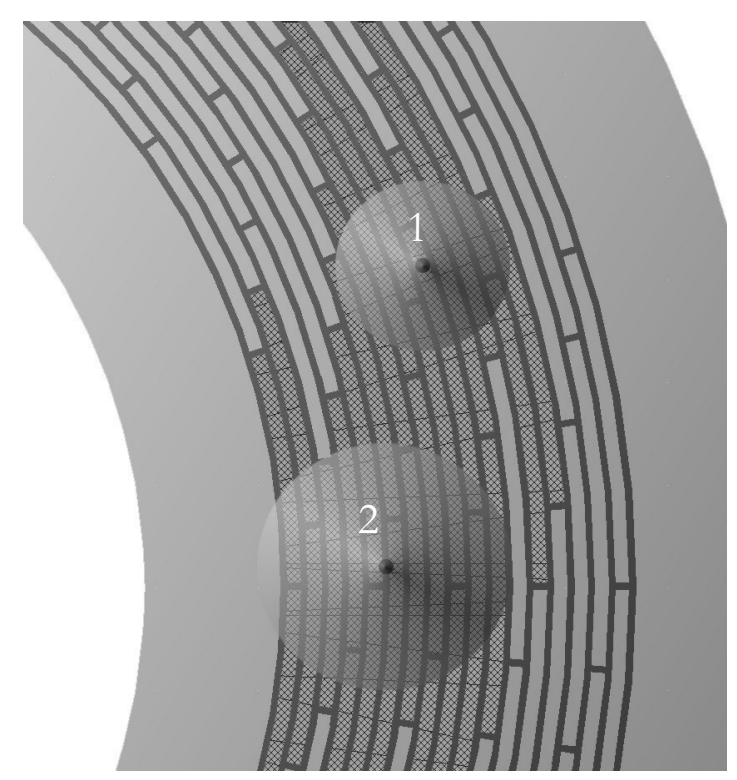

a)

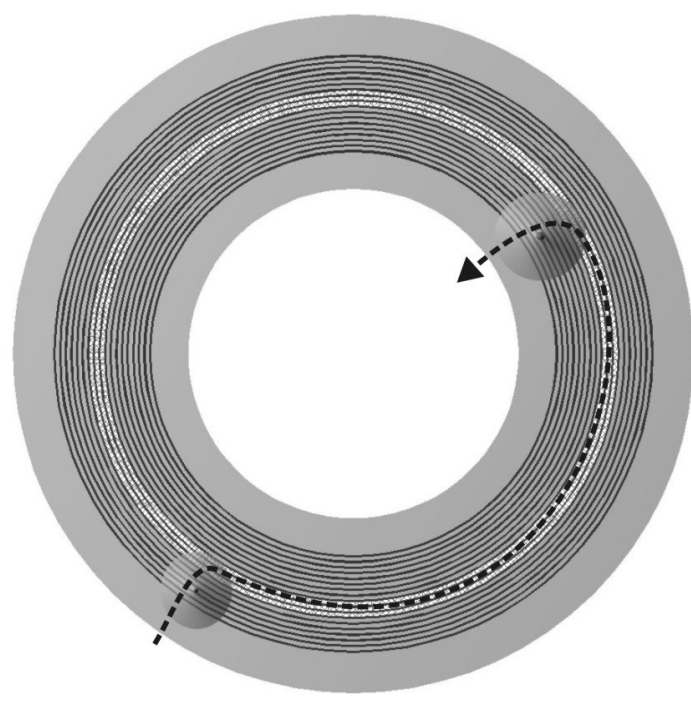

b)

Figure 5-14: Top view: encapsulated particles under the membrane. a) with compartments b) without compartments; the dashed arrow shows the leakage path

When the amount of trapped particles increases, the chance for leakage increases. One can increase the area of the valve seat pattern to be able to encapsulate more particles. This area can be increased by adding valve seats or by increasing the distance between the valve seats. When assuming a viscous flow, particles travel in a straight line inwards to the outlet in radial direction. The time that particles spend above the valve seat pattern is then linearly dependent on the difference between the outer- and inner radius of the valve seat area. Thus, for a larger valve seat area, particles reside longer in this area, increasing the chance that they get trapped under the membrane. But since the area increases with the square of the radius, a doubling of the outer radius would mean that the amount of particles that can be encapsulated becomes four times larger. Thus, increasing the valve seat area works in favor. However, when looking at this from a fabrication perspective; the larger the membrane which covers the valve seat area, the weaker its strength. The size of the valve seat area is constraint by the membrane strength.

To reduce the chance that a particle gets trapped on top of a valve seat, the area of the valve seats themselves should be kept as small as possible. Also, the shape of the valve seats can help to avoid particles sticking on top of them as is depicted in Figure 5-15. A triangular or round shape would be optimal since particles tend to 
slide towards a lower position in between the valve seats. An extremely sharp valve seat might damage the membrane.

This same technique can be used to make it unfavorable for a particle to stay on top of an anchor point. Figure 5-15.a shows a close up of an anchor point. Figure 5-15.b shows a cross-section along the dashed line. The silicon boss is resting on the anchor point. When a particle is in between the silicon boss and the anchor point, leakage is the result as also shown in Figure 5-11. When the surface of the anchor point is ribbed a particle tends to slide in between the ribs and a leak is avoided.

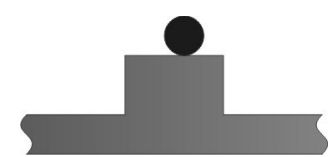

a)

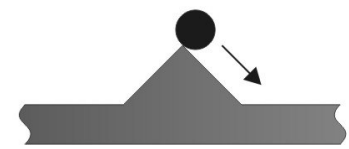

b)

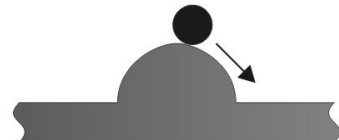

c)

Figure 5-15: V alve seat shapes. a) rectangular b) triangular c) round

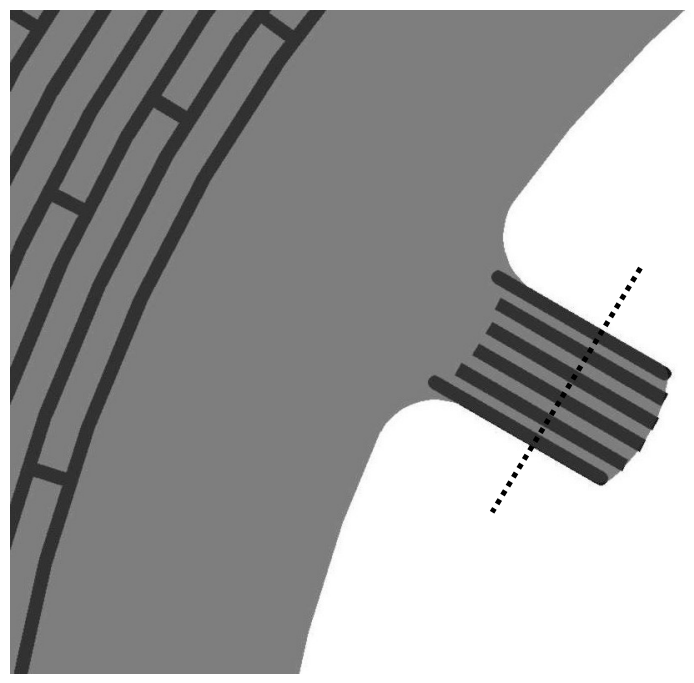

a)

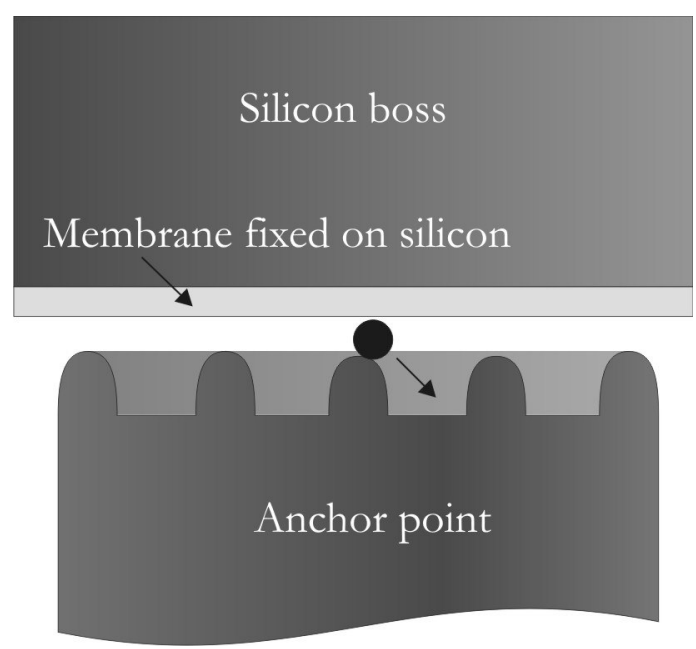

b)

Figure 5-16: a) Close up of an anchor point b) round 


\subsubsection{Valve dimensions}

In this section the dimensions of the spiral spring, the membrane and the valve seat area are determined. It is stressed that the given calculations are based on oversimplified considerations, but in first order the presented constraints are believed to be correct.

\section{- Spiral spring dimensions}

There are a couple of constraints regarding the dimensions of the valve. In chapter 2 a design guideline has been derived for the maximum force that is allowed in the open state of the valve. The valve is open when the membrane is pulled upwards for a distance of $16.8 \mu \mathrm{m}$ and the allowed force to do this is $0.3 \mathrm{~N}$. The spring constant should be as such that this restriction is met. By using Hooke's law the spring constant is calculated:

$$
F_{p}=-F_{s}=k \delta
$$

Where $F s$ is the force exerted by the spring, $k$ is the spring constant and $\delta$ the deflection. $F_{p}$ is the force the piezo-disk has to deliver against the spring. The calculated spring constant is then $0.3 / 16.8 \cdot 10^{-6}=18 \mathrm{kNm}^{-1}$.

The stiffness of the spiral spring for pure bending is calculated by:

$$
K=n E_{s} v\left(\frac{t_{s}}{l_{s}}\right)^{3}
$$

Where $n$ is the number of spiral beams, $E$ the Young's modulus, $v$ the width, $t$ the thickness and $l$ the length of the beams as indicated in Figure 5-17. When the number of rotations of the spiral is given by $m$ the length of a beam is calculated by:

$$
l_{s}=\pi m\left(a_{i}+a_{0}\right)
$$

The torsion of the spiral spring is neglected in the model which results in an overestimation of the stiffness.

Two things play a role in choosing the dimensions. First of all, the width of the etched trench (w) should be comparable with other etched features to avoid problems with the RIE-lag effect [19]. This effect is also discussed in section 4.3.1. Second, enough space should be available to attach the piezo-disc to the silicon in between the outer part of the embossed membrane and the spiral spring as indicated by the diagonal patterned area in Figure 5-17. 
The following dimensions are chosen. The inner $\left(a_{i}\right)$ and outer $\left(a_{0}\right)$ radius of the spring are chosen to be $1800 \mu \mathrm{m}$ and $2820 \mu \mathrm{m}$ respectively. The number of spiral beams $(n)$ is 5 , the height of the beams $(t)$ is $380 \mu \mathrm{m}$ and the Young's modulus is $130 \mathrm{GPa}(<100>$ silicon). The number of rotations of the spiral $(\mathrm{m})$ is 0.56 . The calculated spring stiffness is then $14 \mathrm{kNm}^{-1}$ which is below the maximum allowed stiffness of $18 \mathrm{kNm}^{-1}$.

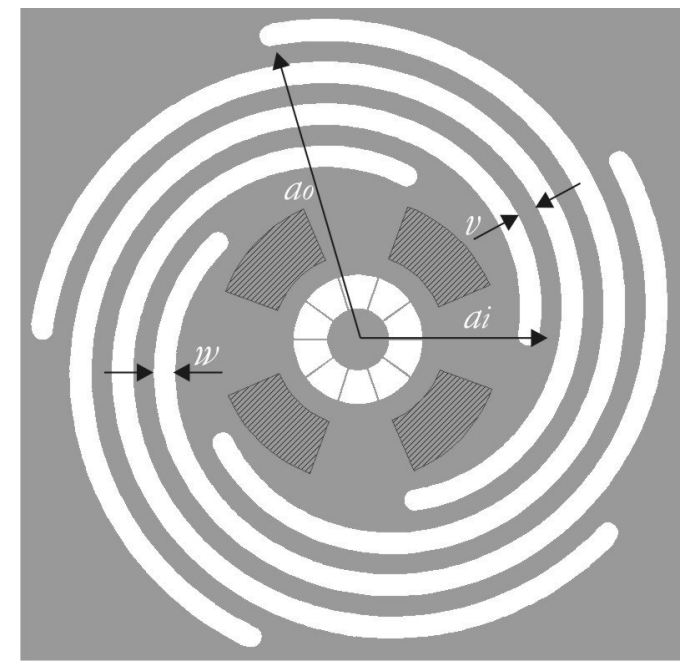

Figure 5-17: Dimensions of the spiral spring

- Membrane dimensions

When the valve is closed the membrane is pulled over the valve seats by the spring mechanism. The spring mechanism is deflected a little bit and thus exerting a force on the membrane to close the valve. The deflection of the spring is determined by the thickness of the membrane as can be seen in Figure 5-18.

Let's assume for the moment a membrane thickness of $0.5 \mu \mathrm{m}$. The force that the spring is exerting at a deflection of $0.5 \mu \mathrm{m}$ is then $7 \mathrm{mN}$ as calculated with equation (5.1). With this force the membrane has to be pulled over the valve seats.

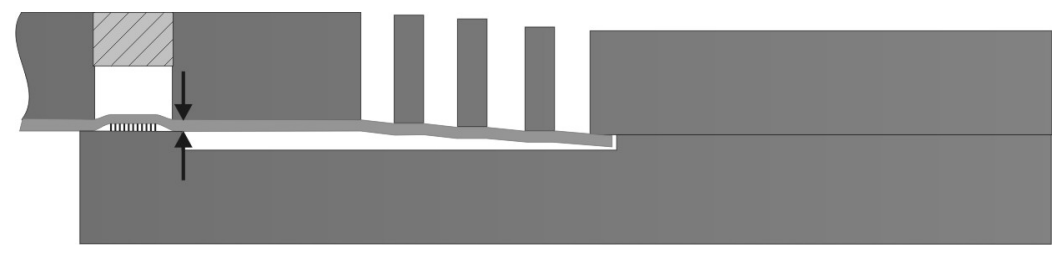

Figure 5-18: Deflection of spiral spring caused by membrane thickness 
To calculate the required force the membrane is divided in three sections. The sections are indicated in Figure 5-13 and Figure 5-21.a as 1, 2 and 3. In section 1 and 2 the membrane is bending around the valve seat area. Section 3 is the part covering the valve seats. We focus on sections 1 and 2 and consider the sections as separate membranes independent of each other. It is also assumed that the initial stress in the membrane is zero which is not self-evident as is pointed out in section 5.4.3.

For large deflections the relation between the applied force and the amount of bending is non-linear. The non-linear deflection curve for a double clamped beam, as depicted in Figure 5-19.a, is given by [20]:

$$
x(y)=\frac{F y}{2 E_{m} b\left(2 t_{m}\right)^{3}}\left(3 l_{m}^{2}-16 y^{2}\right)-\frac{9 F^{3} l_{m}^{4} y}{200 E_{m}{ }^{3} b^{3}\left(2 t_{m}\right)^{11}}\left(5 l_{m}^{2}-16 y^{2}\right)^{2}+\ldots[\mathrm{m}]
$$

Where $x$ is the deflection, $y$ the position on the beam, $l_{m}$ the length, $E_{m}$ the Young's modulus, $t_{m}$ the thickness, $b$ the width and $F$ the force exerted on the beam. The configuration for the membrane is shown in Figure 5-19.b and Figure 5-20. Due to symmetry only half of the force as calculated by equation (5.6) is required to bend the membrane. To give an approximation for the required force for bending the membrane the problem is simplified. As depicted in Figure 5-21.b, it is assumed that the membrane is a straight strip with a width (b) of $2 \pi a 1$. It is a beam with a very large width compared to its length. The deflection at $l_{m} / 2$ is then given by:

$$
x\left(l_{m} / 2\right)=\frac{1}{32} \frac{F l_{m}}{E_{m} \pi a_{1} t_{m}{ }^{3}}\left(3 l_{m}^{2}-4 l_{m}^{2}\right)-\frac{9}{3276800} \frac{9 F^{3} l_{m}{ }^{9}}{E_{m}{ }^{3} \pi^{3} a_{1}{ }^{3} t_{m}{ }^{11}}+\ldots[\mathrm{m}]
$$

The following dimensions are chosen: $l_{m} / 2$ equals $62 \mu \mathrm{m}$ and $t_{m}$ is $0.5 \mu \mathrm{m}$. The height of the valve seats determines the deflection of the membrane as depicted in Figure 5-20. The valve seats are $1 \mu \mathrm{m}$ high (b), which is a suitable thickness considering the fabrication method as is discussed in section 5.3.2. The membrane consists of $\mathrm{SiO}_{2}$ and $\mathrm{Si}_{3} \mathrm{~N}_{4}$ as is discussed in section 5.4.3. The Young's modulus of such a membrane is calculated by:

$$
\frac{\left[Y_{S_{3} N_{4}} /\left(1-v_{S i_{3} N_{4}}\right)\right] \cdot t_{S i_{3} N_{4}}+\left[Y_{S_{i O}} /\left(1-v_{S i O}\right)\right] \cdot t_{S i O_{2}}}{t_{S i_{3} N_{4}}+t_{S i O_{2}}}
$$


Where $Y$ is the young's modulus, $v$ the Poisson's ratio and $t$ the thickness of the layer. The Young's of $\mathrm{Si}_{3} \mathrm{~N}_{4}$ and $\mathrm{SiO}_{2}$ is $400 \mathrm{GPa}$ and $70 \mathrm{GPa}$, respectively and the Poisson's ratio is 0.28 and 0.17 , respectively. For now we assume the membrane is composed of $100 \mathrm{~nm} \mathrm{Si}_{3} \mathrm{~N}_{4}$ and $400 \mathrm{~nm} \mathrm{SiO}_{2}$ the calculated Young's modulus is 180GPa.

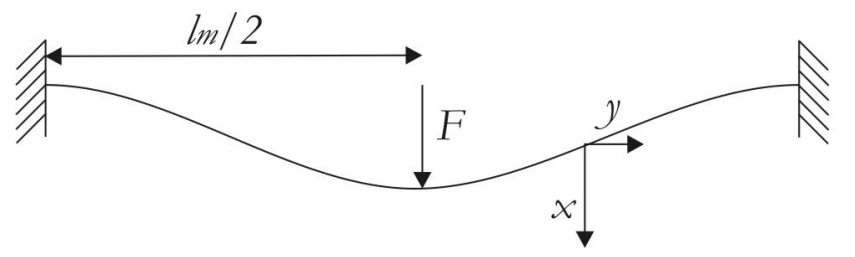

a)

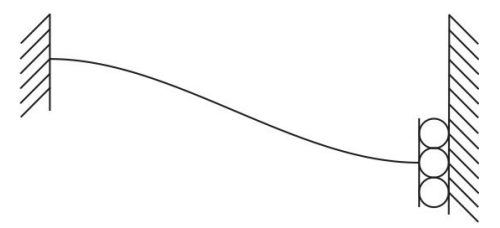

b)

Figure 5-19: a) double clamped beam b) configuration for the membrane

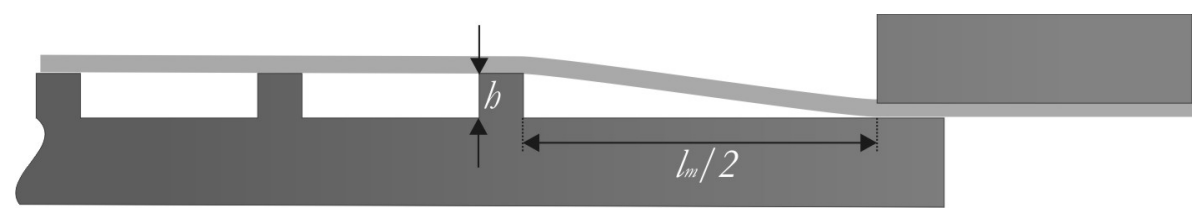

Figure 5-20: Close-up of the bended membrane.

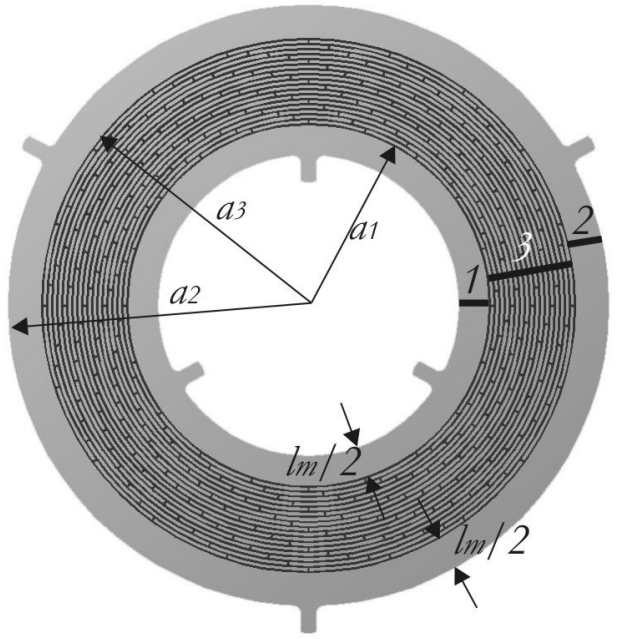

a)

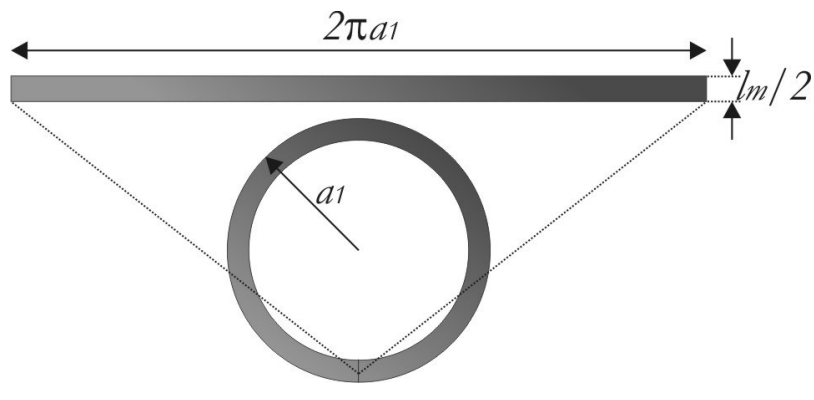

b)

Figure 5-21: a) Bottom wafer valve seat dimensions b) Simplified membrane configuration

The membrane is pulled over the valve seat at two sides as indicated by 1 and 2 in Figure 5-21.a. For section 1 and 2 the radii of the membrane are $a_{1}$ and $a_{2}$ respectively, having dimensions $362 \mu \mathrm{m}$ and $600 \mu \mathrm{m}$ respectively. The inner radius is 
determined by the diameter of the outlet which is chosen to be $600 \mu \mathrm{m}$. This is smaller than the $773 \mu \mathrm{m}$ that is required for the upper end of the thrust range $(10 \mathrm{mN})$. During the course of the project the thrust range was determined while the first valves were already fabricated with a $600 \mu \mathrm{m}$ diameter inner radius. For larger thrust levels the dimensions of the valve can easily be adjusted.

For sections 1 and 2, the required force is calculated by equation (5.7) and added. The total force required to bend the membrane over the $1 \mu \mathrm{m}$ high valve seat pattern is $2.2 \mathrm{mN}$. The first term of equation (5.7) gives the biggest contribution; the second term adds less than $1 \%$. Since the formula is an approximation obtained by a Taylor series this means that the error is less than $1 \%$. The $2.2 \mathrm{mN}$ force is an overestimation because in reality sections 1 and 2 are not clamped at both sides but are connected by section 3. The tension which is building up in sections 1 and 2 is actually distributed over all three sections. This means the stretching in section 1 and 2 is actually less and thus requires less force to bend. The required force is well below the $7 \mathrm{mN}$ that is exerted by the spring.

Now we focus on section 3. In this section particles are encapsulated by the membrane as is depicted in Figure 5-13 and Figure 5-14. It is assumed that this section is not influenced by the other two sections. Furthermore, we simplify the situation by assuming that a trapped particle causes the membrane to bend in a circular way as indicated in Figure 5-13. The relation between the pressure and the membrane deflection is described by [21]:

$$
p=c \cdot x+d \cdot x^{3}
$$

Where

$$
\begin{aligned}
c=c_{b}+c_{i} & =\frac{16}{3\left(1-v^{2}\right)} \frac{E_{m} t_{m}^{3}}{a_{m}^{4}}+\frac{4 t_{m} \sigma_{0}}{a_{m}^{2}} \\
d & =\frac{7-v}{3(1-v)} \frac{E_{m} t_{m}}{a_{m}^{4}}
\end{aligned}
$$

Here, $x$ is the deflection at the centre, $v$ is the Poisson's ratio, $E$ the Young's modulus, $\sigma_{0}$ the initial stress in the membrane, $p$ is the pressure difference, $a_{m}$ is the radius of the deflected part of the membrane and $t_{m}$ is its thickness. For small deflections in comparison with the thickness of the membrane, relation 
(5.10) is linear and the second term of equation (5.9) is negligible. In this case the membrane mainly bends. We assume that the initial stress in the membrane is zero by which the second term of equation (5.10) drops out. For large displacements, the deflection scales with the cubic root of the pressure. In this regime the tension in the membrane plays a significant role.

We plotted the radius of opening as a function of the deflection of the membrane at a tank pressure of 1 bar, which is the lowest operation pressure. This is the worst case since higher tank pressures will cause the radius of the deflected membrane to shrink. The smaller the radius the less valve seat compartments are compromised and thus less chance for leakage. For the calculation we used the following values; a membrane thickness of $0.5 \mu \mathrm{m}$, a Young's modulus of 180GPa and a Poisson's ratio of 0.27 . The plot is shown in Figure 5-22. For a particle with a size of $2 \mu \mathrm{m}-$ which causes a $2 \mu \mathrm{m}$ deflection - this results in an opening with a radius of $70 \mu \mathrm{m}$, thus a diameter of $140 \mu \mathrm{m}$. The width of the valve seat area should be larger than $132 \mu \mathrm{m}$ to be able to encapsulate a $2 \mu \mathrm{m}$ particle. We chose a width of $173 \mu \mathrm{m}$.

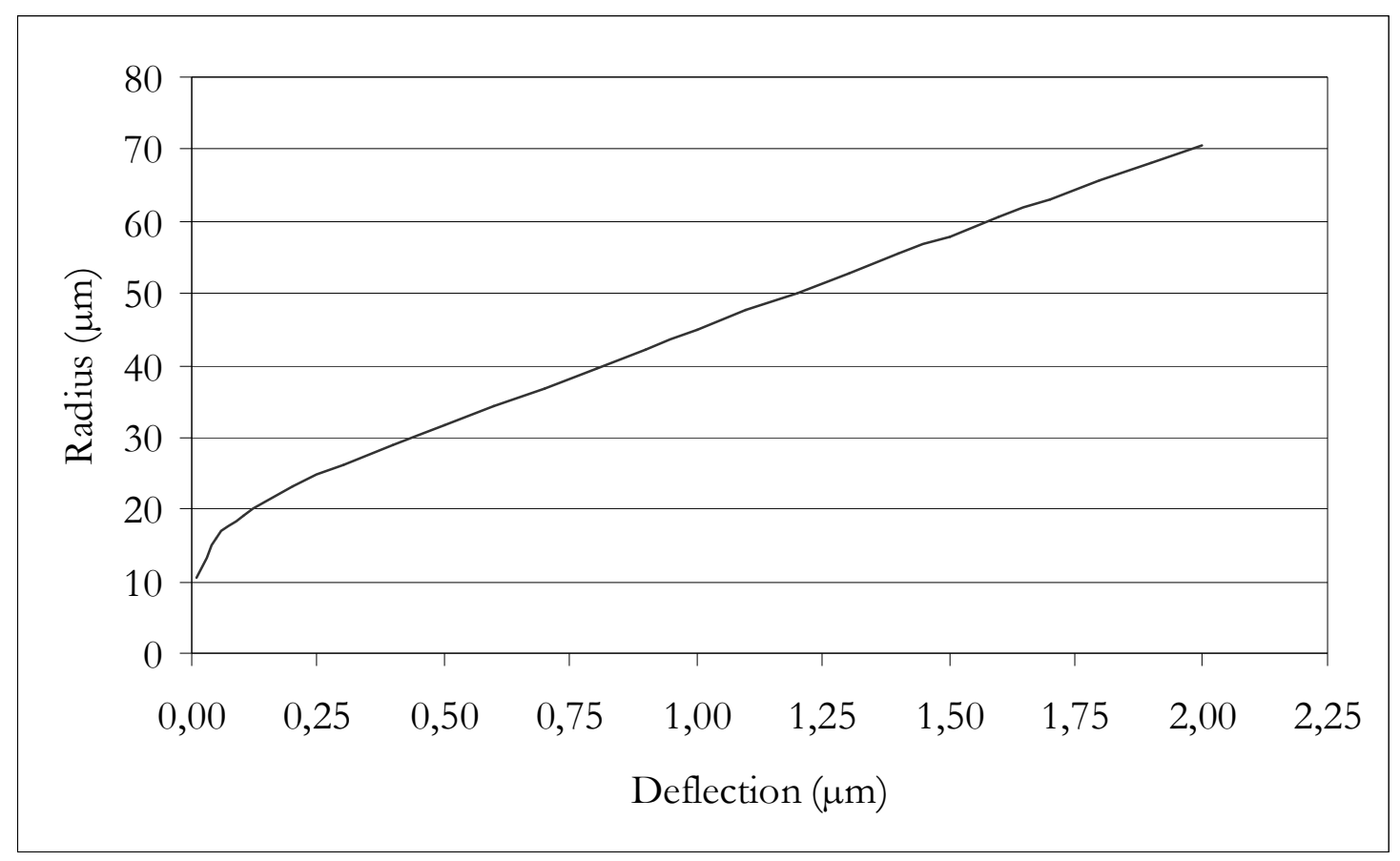

Figure 5-22: Radius of the opening as a function of membrane deflection at 1 bar tank pressure 
A final remark is made about the opening of the valve. In the closed state the tank pressure acts on the membrane and the boss. The outer radius of the membrane is $600 \mu \mathrm{m}$ which agrees with the outer radius of the valve seat as indicated in Figure 5-21.a by $a_{2}$. The force due to a tank pressure of $4.5 \mathrm{bar}$ is calculated to be $0.5 \mathrm{~N}$ :

$$
F_{t}=\pi\left(600 \cdot 10^{-6}\right)^{2} \cdot 4.5 \cdot 10^{5}=0.5 \mathrm{~N}
$$

In the closed state the piezo-disc is not deflected and can exert a force of $2.4 \mathrm{~N}$ which is sufficient to open the valve. At the moment the valve is opened the pressure over the membrane reduces significantly and plays a minor role. An indication of the pressure drop over the valve in the open state is given in chapter 2.

\subsection{Fabrication and results}

In this paragraph the fabrication of the membrane valve is presented. The valve is based on bulk micro machining of silicon and on thin film micro machining techniques. The valve seat and chamber are shaped in the bottom wafer. The top wafer contains the spring mechanism and a thin film membrane which closes the valve. These two parts are fabricated separately and at last fusion bonded. Some baseline technologies are discussed in section 5.4.1. Then several manufacturing routes are compared for making valve seat in section 5.4.2. Finally, in section 5.4.3, the fabrication of the membranes is explored in depth.

\subsubsection{Baseline technologies}

In this section three baseline technologies are discussed specified at the valve fabrication. First deep reactive ion etching is considered followed by fusion bonding and finally local non-bonding.

\section{- DRIE etching through the wafer}

Deep reactive ion etching is used to make $2 \mathrm{D}$ extruded shapes in the silicon. It is used for the bottom wafer - the valve seat wafer - to etch the valve chamber and outlet. For the top wafer - the membrane wafer - it is used to shape the spiral spring. Also, by etching through the wafer and selectively stopping at the membrane the free hanging membrane is obtained. The free etching of this membrane is not straightforward. As is shown in Figure 5-23.a the boss in the 
middle of the membrane is connected with the outer part by beams. The light grey part is the membrane. When etched through, the membrane should be free hanging; the beams should not be connected to the membrane. This can be obtained by etching with a slight negative taper. This is depicted in cross sectional view in Figure 5-23.b. A beam with a width of $20 \mu \mathrm{m}$ is etched $380 \mu \mathrm{m}$ in depth with a slight negative taper. This results in a beam with a wedge shape and a free hanging membrane.

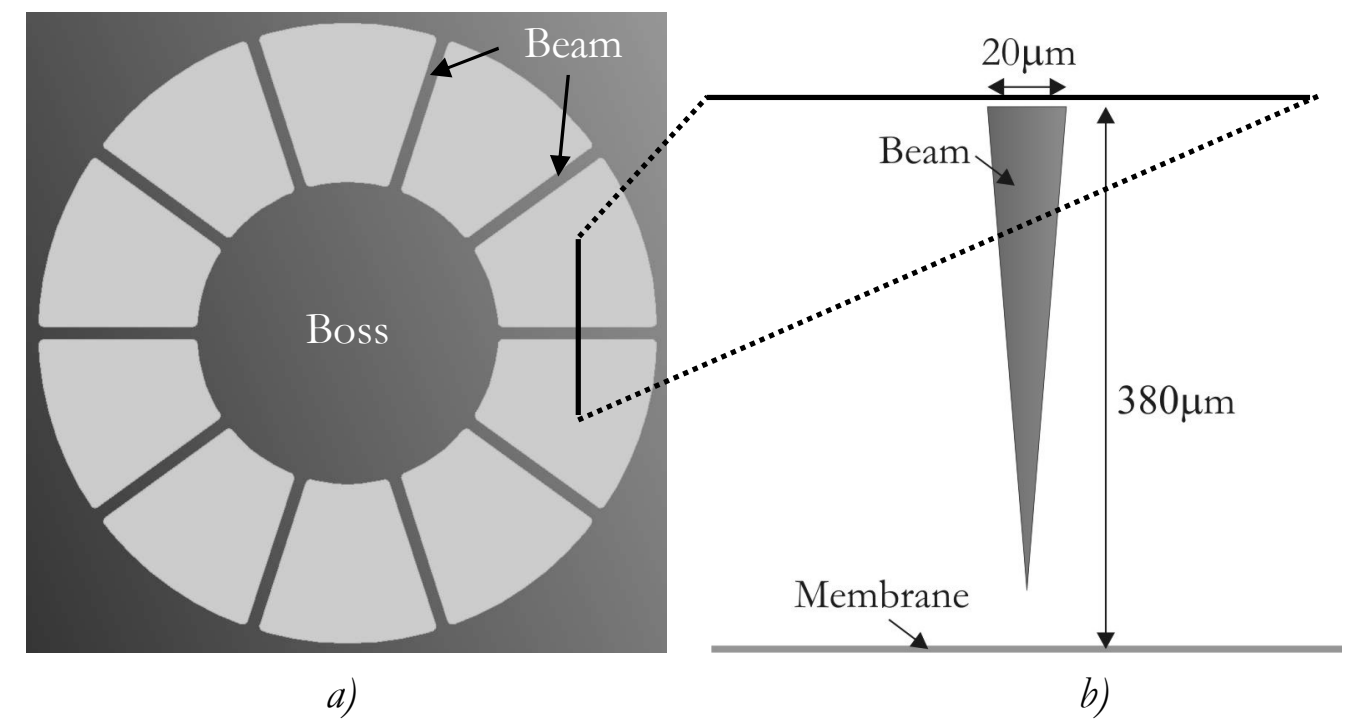

Figure 5-23: a) Top view of the middle part of the valve b) Cross section of a beam

One can also use the notching - also called footing - effect if the stopping layer, in this case the membrane, is an electrically isolating layer [22]. Charged particles are accelerated towards the membrane and encounter an isolating layer, for example $\mathrm{SiO}_{2}$. This layer is charging under the bombardment of charged particles. When the stopping layer gets charged the incoming charged particles will be deflected towards the silicon sidewall of an etched feature due to electrostatic forces. The passivation layer is removed from the sidewall of the silicon beam near the membrane. There where the passivation layer is removed the beam is etched by the etching radicals. This results in the profile as shown in Figure 5-24. 


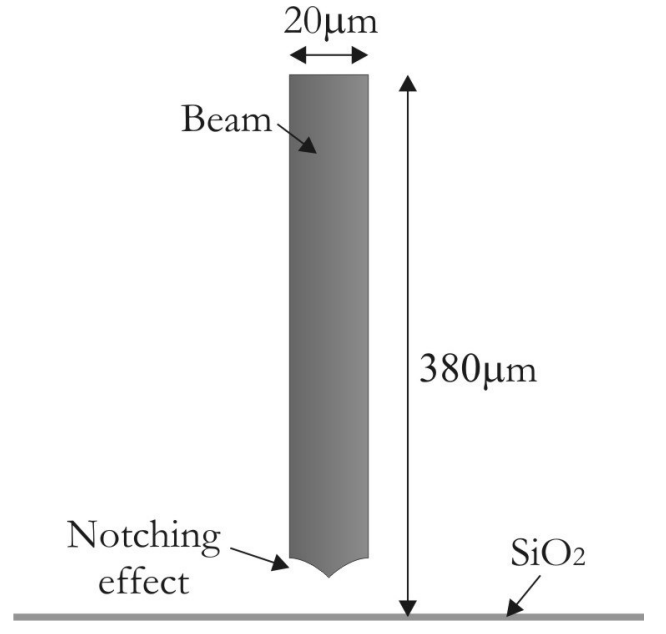

a)

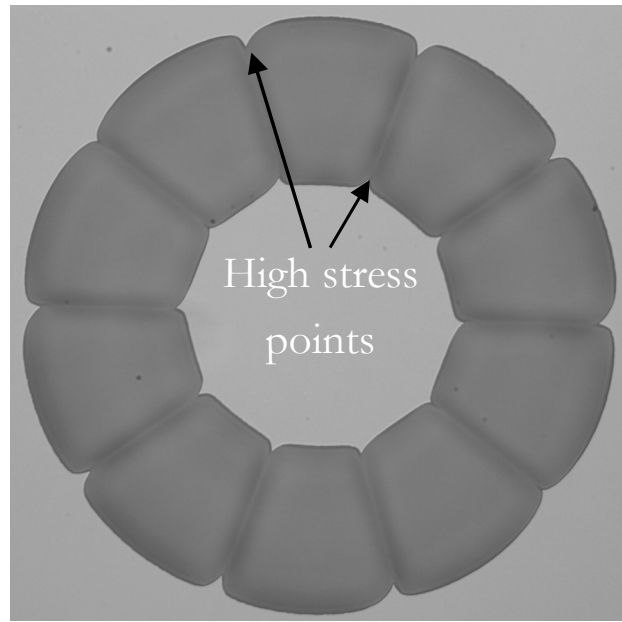

b)

Figure 5-24: a) Cross section of a beam directionally etched and stopping at $\mathrm{SiO}_{2}$ b) Flower shaped membrane

We will use $\mathrm{SiO}_{2}$ as stopping layer and use the notching effect to obtain a free hanging membrane. Compared with the negative tapered etching technique the notching technique results in a beam with more bulk material and thus a stronger connection between the boss and outer part. However, due to the notching the membrane is shaped like a flower instead of a circular circumference. The shape is shown in Figure 5-24.b. This shape causes an uneven stress contribution when the membrane is pulled over the valve seats. A higher stress concentration is expected at the sharp points.

Etching through the wafer is also used to obtain circular devices, ready to be bonded to the glass tubes. A wafer contains 16 devices as shown in Figure 5-25.a and they need to be separated to obtain a single valve. To separate these devices dicing is not an option because of the circular shape. After etching through the wafer, several other fabrication steps need to be done and thus the devices should still be in the framework of the wafer. We use small break beams as indicated in Figure 5-25.b to hold everything together. When the final fabrication step is done the devices can be separated by breaking them out of the framework. 


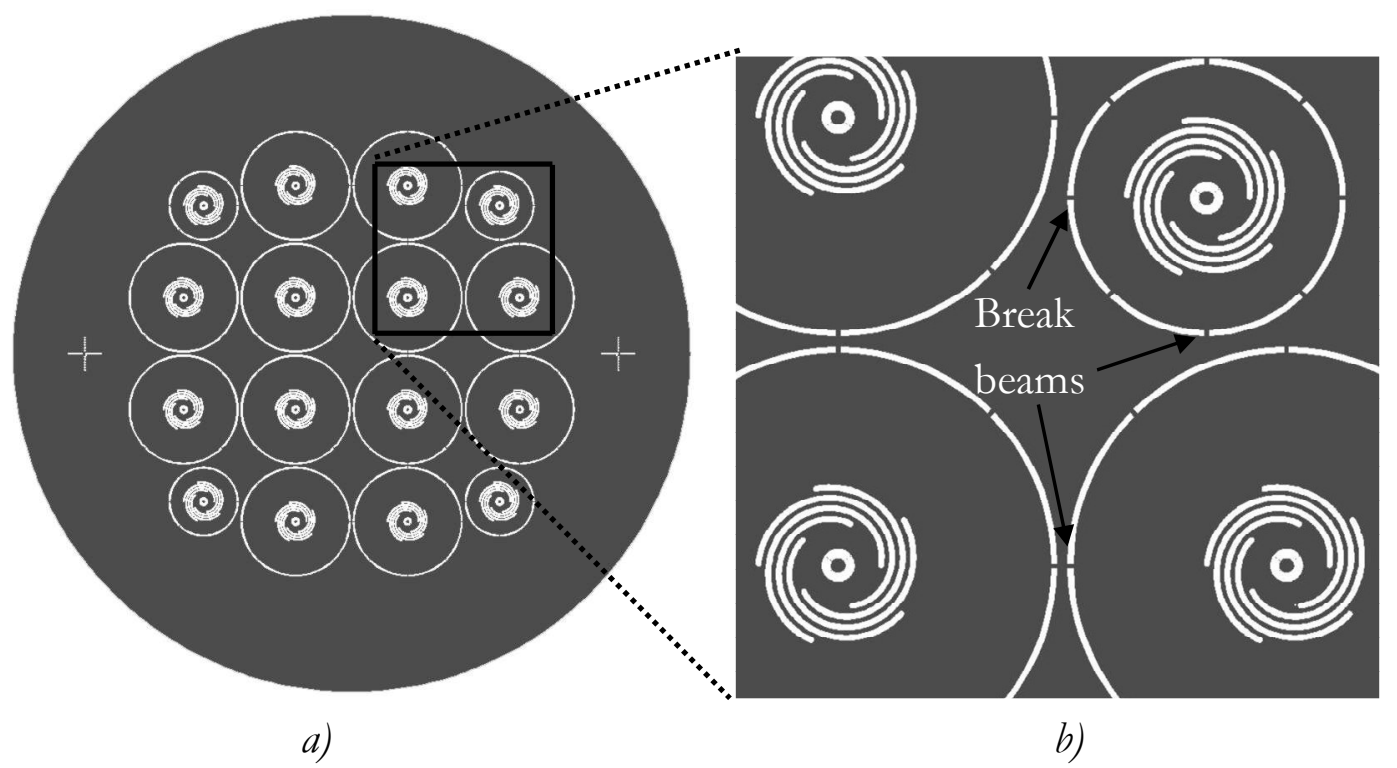

Figure 5-25: a) Top view of the 16 devices on a wafer (4 smaller ones for testing) b) Zoom in on the break beams

When etching the wafer with DRIE the wafer temperature is maintained constant by Helium backside cooling [19]. The Helium pressure underneath the wafer is typically $1000 \mathrm{~Pa}$ while the pressure on the topside of the wafer is much lower. A typical process pressure is $4.5 \mathrm{~Pa}$. The pressure difference over the wafer is causing the wafer to bend upwards. Care must be taken with the decrease in rigidity of the wafer when it is etched through. The wafer is weakened and should not break under the Helium backside pressure and thus the break beams should be rigid enough to avoid this. The effect on the wafer temperature due to the bending is discussed in [19]. The wafer temperature is important to maintain directionality and avoid under-etching. The silicon is etched with a high speed etch recipe with an ICP power of $2500 \mathrm{~W}$. When the bottom of the wafer is reached and the beams are etched due to notching, the thermal control of the beams is compromised. The thermal impact from the plasma source heats up the beams which results in a degradation of the etch inhibiter and thus under-etching is the result. In that case it is possible to reduce the ICP power to $1500 \mathrm{~W}$, at the final stage of etching, in order to decrease the thermal impact and avoid under-etching.

Helium leakage should be avoided when the wafer is etched through at the break beam locations. This can be done in several ways. A carrier wafer can be used underneath the device wafer. Fomblin oil which is spin-coated on this carrier wafer 
is used to attach it to the device wafer. Fomblin oil is used as a lubricant for vacuum pumps and does not evaporate in a low pressure environment. Another method to avoid Helium leakage is the use of thin films. A thin layer can be applied on the backside of the wafer which is then used as stop layer for etching and barrier against Helium leakage. This layer has to be strong enough - and thus thick enough - to avoid rupture of the layer due to the Helium backside pressure. We use a $20 \mu \mathrm{m}$ thick foil which can be laminated on the device wafer; it is removed by $\mathrm{HNO}_{3}$ afterwards. This foil also gives some rigidity to the framework to avoid breaking of the wafer due toe the Helium pressure. Thinner layers might also be used, like spincoated photo-resist or polyimide.

\section{- Fusion bonding - Si-Si}

The valve consists of two wafers. They are fabricated separately and finally the two parts are fusion bonded. The fusion bonding process is the most critical step in the fabrication process. In general the following procedure is used. First the two wafers are cleaned thoroughly. Then the native silicon oxide is stripped and the wafers are treated with Piranha. In this solution of Sulfuric acid and Peroxide a fresh thin film of silicon oxide is grown. When pulled out of this solution the bonding surface is very reactive. Then the two wafers are aligned and placed in proximity. When the surfaces locally touch a pre-bond propagates. After the pre-bond the wafers are annealed at $1100^{\circ} \mathrm{C}$ and fused together.

For a successful pre-bond a couple of things are important. First of all it is important that the bonding surface is virginal. It is essential to protect the surface during processing. A good strategy for this is to grow a thin film of $\mathrm{SiO}_{2}$ as protective layer. This layer is grown on brand new silicon wafers. Then the wafers are processed, and finally, just before the bonding process, the $\mathrm{SiO}_{2}$ layer is removed.

Another important requirement for a successful pre-bond is the absence of particles on the bonding surface. Any particle on the surface will cause a void in between the wafers and can cause a defective device. It is therefore very important that no residues are left from earlier process steps. This should be kept in mind during the shaping of the thin films and silicon bulk. The shaping of the bulk silicon is done by deep reactive ion etching. In this process an etch inhibitor is introduced to obtain directionality in the etch profile. This inhibitor leaves fluorocarbon residues 
which need to be removed before bonding. This is done by burning the fluorocarbon residues at $800^{\circ} \mathrm{C}$. A thin layer of $\mathrm{SiO}_{2}$ is grown at this temperature which is removed in HF and any last residue will lift off.

For our particular valve another factor is influencing the pre-bonding process. When the two wafers are put in proximity and pushed together to touch, the spring mechanism is pushed in as is shown in Figure 5-7. The spring is pushing the two wafers away from each other. The pre-bond force should be much larger than the force exerted by the spring. Values for the pre-bond strength can be found in [23]. A safe value for the bond strength $\left(p_{b}\right)$ is $1 \mathrm{MPa}$. In Figure 5-10.a the bonding area is indicated by the diagonal patterned area having an inner- $\left(r_{i}\right)$ and outer $\left(r_{0}\right)$ radius of $3.3 \mathrm{~mm}$ and $8 \mathrm{~mm}$ respectively. For a single valve the pre-bond strength is obtained by:

$$
F_{b}=p_{b} \cdot \pi\left(r_{o}^{2}-r_{i}^{2}\right)=1 \cdot 10^{6} \cdot \pi\left(\left(8 \cdot 10^{-3}\right)^{2}-\left(3.3 \cdot 10^{-3}\right)^{2}\right)=167 N
$$

The calculated value is $167 \mathrm{~N}$ which is much larger than the $7 \mathrm{mN}$ that is exerted by the spring as calculated in section 5.3.2. It is expected that this will not cause any problem.

\section{- Local non-bonding-Si3 $\mathrm{N}_{4}-\mathrm{Si}_{3} \mathrm{~N}_{4}$}

Due to the high fusion temperature of $1100^{\circ} \mathrm{C}$ most glass like materials tend to stick to each other. The valve seat and membrane should not stick to each other, since it would result in an 'always' closed valve instead of a 'normally' closed valve. To prevent bonding of the active part of the valve we use a technique which is called local non-bonding. Gui et al. showed how the surface roughness is influencing the bonding process [24]. When a surface is rough enough it will not bond. A passive valve has been fabricated by tuning the roughness of a surface to avoid bonding $[25,26]$. This valve has a silicon valve seat and is closed by an embossed silicon sealing. On the sealing a layer of silicon rich nitride is deposited to avoid bonding. When leakage is considered the roughness of the active part of the valve should be as small as possible. On the other hand, to inhibit bonding, the surface should be rough enough. This is a delicate balance and the roughness should be controlled accurately. A silicon rich nitride layer, deposited by LPCVD, can contain particles which will cause leakage [27]. $\mathrm{A} \mathrm{Si}_{3} \mathrm{~N}_{4}$ layer which is deposited by LPCVD does not contain such particles and thus the roughness is better tuned. The roughness of the 
$\mathrm{Si}_{3} \mathrm{~N}_{4}$ layer increases when a thicker layer is grown. Figure 5-26.a shows a close up of a fabricated valve seat. The top wafer has been bonded to the bottom wafer and the spiral spring was later removed as can be seen in Figure 5-26.b. In the close up of the valve seat one can see that the membrane is still present and pulled over the valve seats. The $\mathrm{Si}_{3} \mathrm{~N}_{4}$ membrane is bonded to the silicon next to the valve seats. The bonded areas are encircled by a white line. A cross-section is depicted in Figure 5-26.c to clarify the place were the membrane is bonded to the silicon. If the membrane would not stick it would have been removed with the spring mechanism.

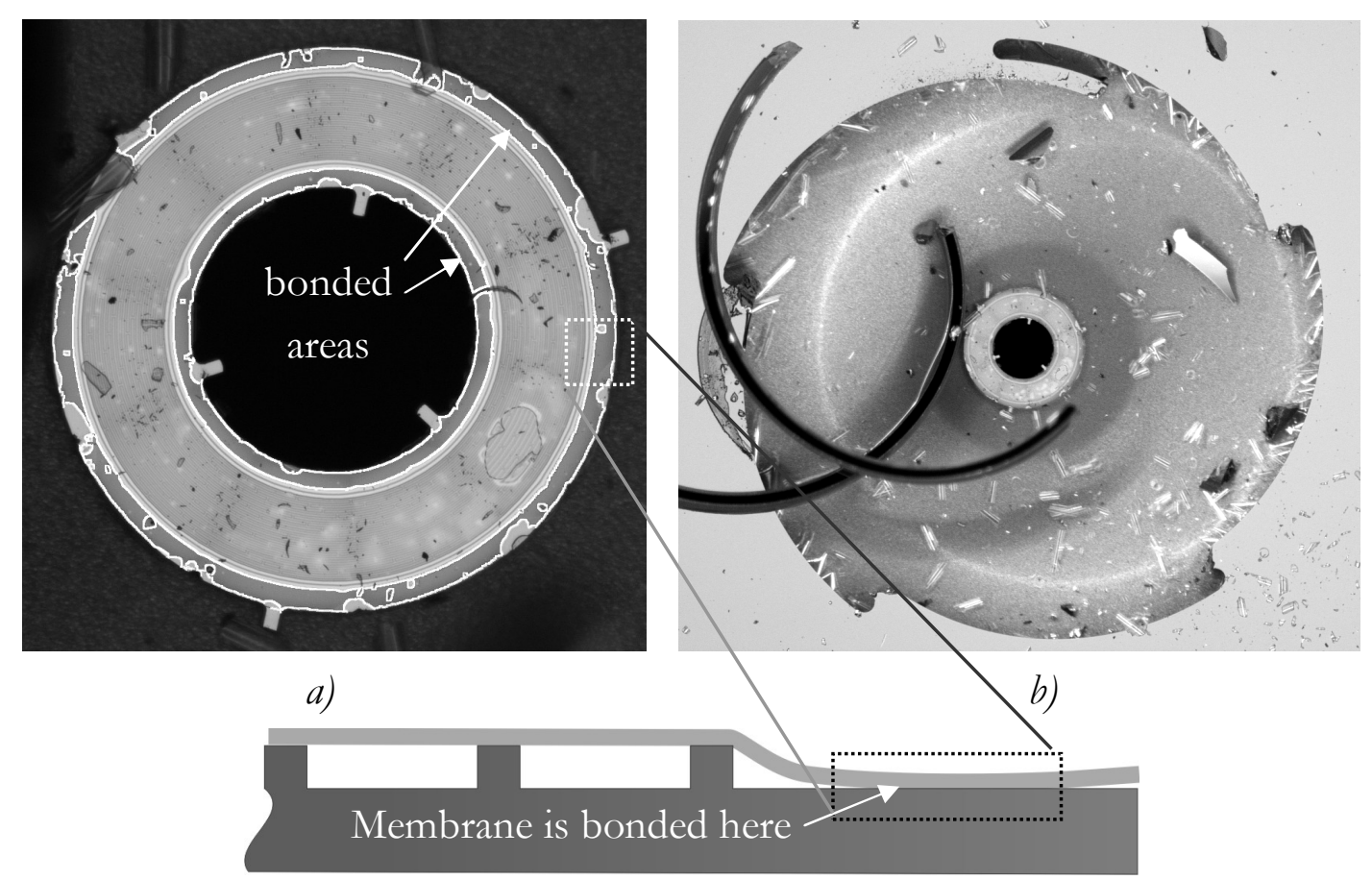

c)

Figure 5-26: a) Membrane bonded next to the valve seat. b) Spiral spring broken out. c) Cross-section of bonded membrane.

Bonding of $\mathrm{Si}_{3} \mathrm{~N}_{4}$ to $\mathrm{Si}_{3} \mathrm{~N}_{4}$ is even better inhibiting the bonding compared to $\mathrm{Si}_{3} \mathrm{~N}_{4}$ to silicon bonding. This is probably caused by the fact that the silicon surface is covert with a thin layer of fresh silicon oxide. This surface is very reactive - due to dangling bonds - and can easily make a bond. On a $\mathrm{Si}_{3} \mathrm{~N}_{4}$ layer almost no oxide is grown and thus is this surface much less reactive. Therefore, not only the valve membrane, but also the whole valve seat area will be covert with a thin film of $\mathrm{Si}_{3} \mathrm{~N}_{4}$. 
A final remark can be made on the fact that the membrane is bonded next to the valve seats. It is an indication that the membrane is really pulled over the valve seats by the spiral spring. Otherwise no bonding would have occurred because the membrane would not have been in contact with the silicon.

\subsubsection{Valve seat wafer fabrication}

This section discusses the fabrication of the valve seats and valve seat wafer. The fabrication scheme of one of the first valve seats that were made is depicted in Table 5-II, although later on optimized. These valve seats are simply rectangular bumps with a height of $430 \mathrm{~nm}$ made by thin film techniques.

1) The process starts with a thermally grown 430nm thick $\mathrm{SiO}_{2}$ layer on a $380 \mu \mathrm{m}$ thick $<110>$ silicon wafer. Then, a $120 \mathrm{~nm}$ thick layer of $\mathrm{Si}_{3} \mathrm{~N}_{4}$ is deposited by low pressure vapor deposition (LPCVD) over the $\mathrm{SiO}_{2}$ layer.

2) The $\mathrm{Si}_{3} \mathrm{~N}_{4}$ and $\mathrm{SiO}_{2}$ layer is patterned with the valve seat features by reactive ion etching with a mask layer of photo-resist. $100 \mathrm{~nm}$ of $\mathrm{SiO}_{2}$ is left on both sides of the wafer as protective layer for the bond surface.

3) Another $40 \mathrm{~nm}$ thick layer of $\mathrm{Si}_{3} \mathrm{~N}_{4}$ is deposited by LPCVD. It is patterned and acts as a bond inhibiting layer.

4) Now we zoom out and show only the left half of the valve. The valve is symmetric and therefore only one half is shown. The wafer is coated with a $500 \mathrm{~nm}$ TEOS, which is patterned with the valve chamber and outlet.

5) In the next step the outlet of the valve is etched. This is the first DRIE step for which a photo-resist mask layer is used. $20 \mu \mathrm{m}$ of silicon is still left standing.

6) The photo-resist is stripped to reveal the TEOS mask layer which is used for the second DRIE step. A carrier wafer - attached by Fomblin oil - is used to prevent Helium leakage during this etch step. The last $20 \mu \mathrm{m}$ of silicon is etched to open the outlet and the valve chamber is shaped.

7) After removing the fluorocarbon and stripping the protective $\mathrm{SiO}_{2}$ layer the wafer is ready to be bonded.

The initial attempt to fabricate this valve seat wafer was a little bit different than shown in the fabrication scheme (Table 5-II). Step 3 was skipped and thus the second $\mathrm{Si}_{3} \mathrm{~N}_{4}$ layer was not present over the valve seats. This resulted in the bonding of the $\mathrm{Si}_{3} \mathrm{~N}_{4}$ membrane to the silicon next to valve seats as shown in 
Figure 5-26.a and as discussed earlier at the end of section 5.4.1. That is why the $\mathrm{Si}_{3} \mathrm{~N}_{4}$ layer is added to introduce a better bonding inhibiting layer.

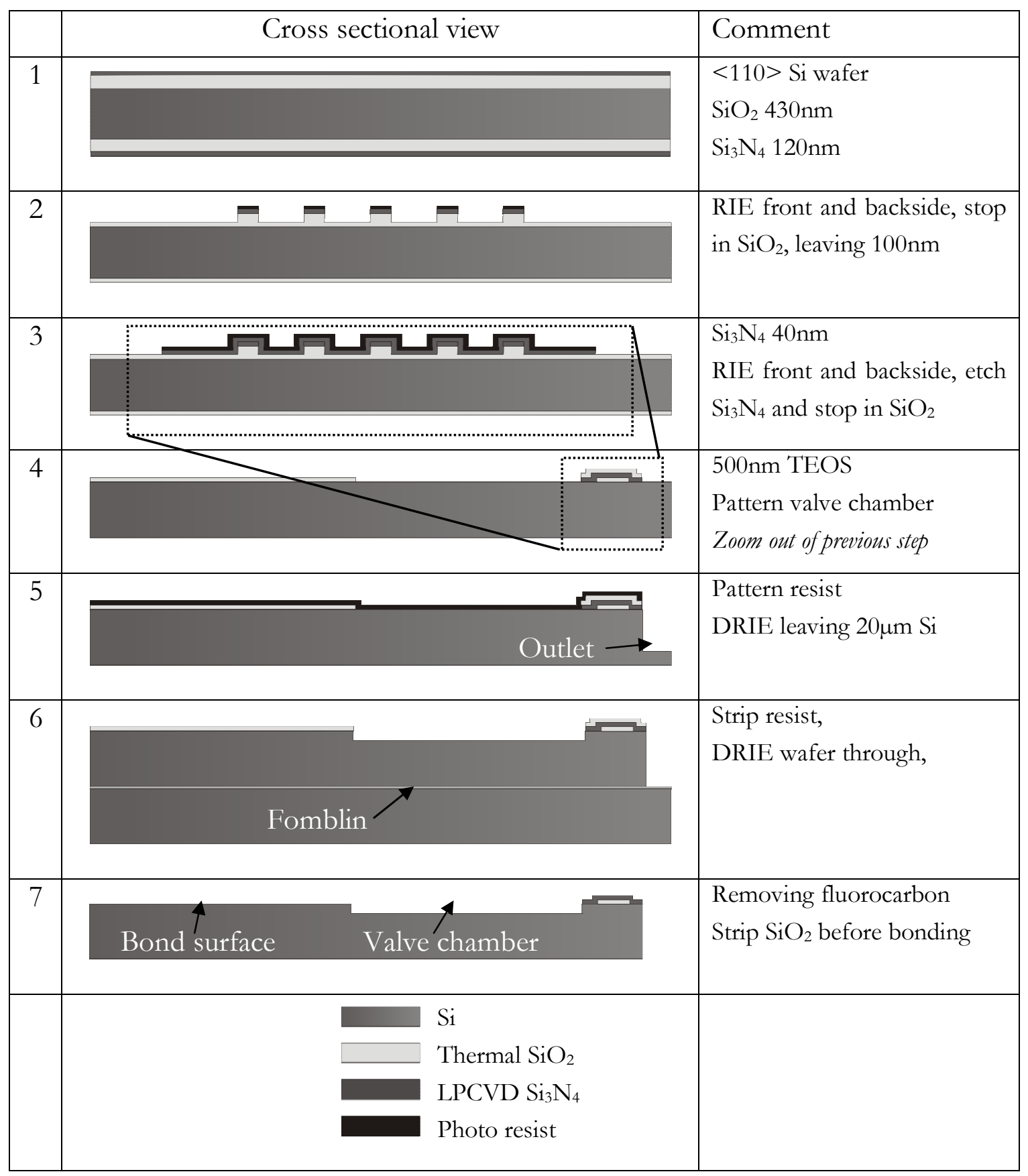

Table 5-II: V alve seat fabrication scheme.

This fabrication process suffered from poor reproducibility. Several valve seat wafers have been fabricated in this way but most of them did not bond well in the end. This is caused by the RIE etching process of the $\mathrm{Si}_{3} \mathrm{~N}_{4}$ and $\mathrm{SiO}_{2}$ layers to 
shape the valve seats. Sometimes residues of this process are left on the wafer surface. The amount of residue depends on the history and state of the etch chamber. A dirty chamber leaves a lot of residue on the wafer, which is sometimes very hard to remove. To ensure a clean process it is decided to shape the valve seats by wet etching instead of the dry etch method. Besides the problems with residues, the shape of valve seats that are processed with RIE is not optimal. It results in a rectangular shape which is not the desired shape as discussed in section 5.3.1. A triangular or round shape is preferred to encourage particles to slide to a position in between the valve seats. A second fabrication method is considered - which is not yet executed - to make round shaped valve seats. The process scheme is shown in Table 5-III.

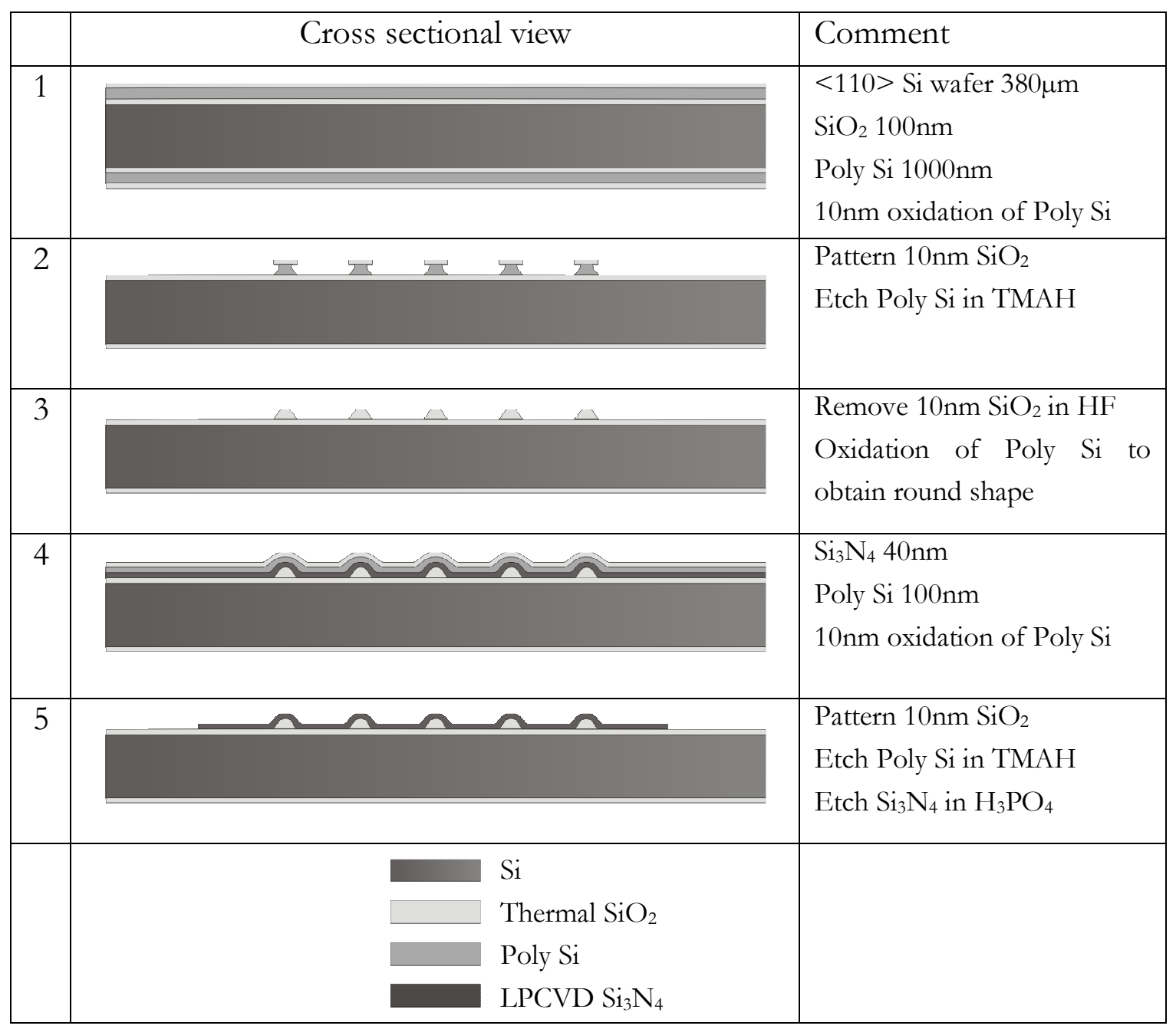

Table 5-III: Round valve seat fabrication scheme. 
The fabrication of these optimized valve seats is as follows.

1) On a $380 \mu \mathrm{m}$ thick $<110>$ silicon wafer a 100 nm thick $\mathrm{SiO}_{2}$ layer is grown. Then $1000 \mathrm{~nm}$ poly silicon is deposited and $10 \mathrm{~nm}$ of the layer is oxidized.

2) The $10 \mathrm{~nm}$ oxide is patterned and is used as a mask layer for the etching of the poly silicon. The wet etching of poly silicon results in a typical isotropic profile. By under etching of the poly silicon the width of the valve seats can be tuned and submicron features can be obtained.

3) The $10 \mathrm{~nm} \mathrm{SiO} 2$ mask layer is stripped in HF and the poly silicon is oxidized. Due to this oxidation step sharp convex corners of the valve seats are rounded $[28,29]$. This effect is shown in Figure 5-27.

4) To avoid bonding of the valve seats a $\mathrm{Si}_{3} \mathrm{~N}_{4}$ layer is applied. A $40 \mathrm{~nm}$ thick LPCVD $\mathrm{Si}_{3} \mathrm{~N}_{4}$ layer is deposited followed by the deposition of $100 \mathrm{~nm}$ poly silicon. 10nm of the poly silicon is oxidized.

5) The $10 \mathrm{~nm}$ thick layer of $\mathrm{SiO}_{2}$ is patterned and is used as a mask layer for the etching of the poly silicon in TMAH. Then the poly silicon is used as a mask layer for the etching of $\mathrm{Si}_{3} \mathrm{~N}_{4}$ in $\mathrm{H}_{3} \mathrm{PO}_{4}$. Further processing of the valve seat wafer is the same as discussed earlier and shown in Table 5-II steps 4 till 7.

The latter fabrication process for the valve seats results in round valve seats which is important to create a bias for a particle to move to a position in between the valve seats. Furthermore, the shaping of the valve seats is done by wet etching only, to ensure a clean surface which is important for the final bonding step. The dry etching steps to shape the silicon wafer did not cause any problem for the bonding. This is done with a better controlled process which leaves much less residue. The fluorocarbon residue that is left after etching is easily removed by burning it at $800^{\circ} \mathrm{C}$ and a consecutive lift off in $\mathrm{HF}$.

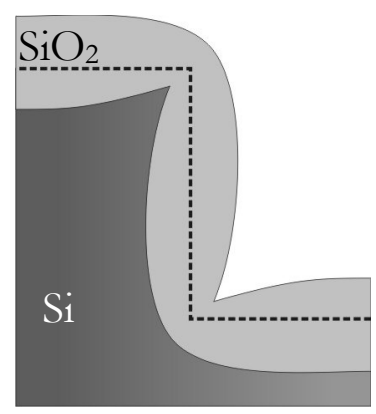

Figure 5-27: Rounded feature due to oxidation. The dashed line indicates the original shape. 


\subsubsection{Membrane fabrication}

This section focuses on the fabrication of the membrane which seals the valve. There are a couple requirements that are important for the membrane. First of all it needs to be covered with $\mathrm{Si}_{3} \mathrm{~N}_{4}$ to avoid bonding of the membrane to the valve seats as discussed in the section 5.4.1. Second, the ideal situation for the membrane would be that it is without stress. As deposited, LPCVD $\mathrm{Si}_{3} \mathrm{~N}_{4}$ has a tensile stress of $1.1 \mathrm{GPa}$ with respect to the underlying silicon substrate. When this material is used as a membrane it is unable to follow surface irregularities as explained previously with the help of e.g. Figure 5-14 and Figure 5-22. The internal stress in the membrane has to be compensated as much as possible, to enable the membrane to comply for any irregularities. It is possible to compensate the stress by making a membrane of two materials; one material being $\mathrm{Si}_{3} \mathrm{~N}_{4}$ which has tensile stress, and another material having a compressive stress, for example $\mathrm{SiO}_{2}$. Thermally grown $\mathrm{SiO}_{2}$ has a compressive stress of $0.3 \mathrm{GPa}$. By tuning the thickness of the tensile $\mathrm{Si}_{3} \mathrm{~N}_{4}$ layer and a compressive $\mathrm{SiO}_{2}$ layer the internal stress can be evened out. Care must be taken with an overcompensated membrane which results in a membrane under compression. It is disastrous when the membrane is under compressive stress since it will buckle. The membrane then becomes fragile and the valve seats are not sealed properly.

\section{- Single side compensated membrane}

The first membrane that has been fabricated consisted of a thermal $\mathrm{SiO}_{2}$ layer underneath a $\mathrm{Si}_{3} \mathrm{~N}_{4}$ layer. We call this the single side compensated membrane. The mean stress in the stack can be calculated by the following equation:

$$
\frac{\sigma_{S_{i} N_{4}} \cdot t_{S i_{3} N_{4}}+\sigma_{S_{i O_{2}}} \cdot t_{S_{i O}}}{t_{S i_{3} N_{4}}+t_{S i O_{2}}}=\frac{1.1 \cdot 120-0.3 \cdot 430}{120+430}=10 \mathrm{MPa}>0
$$

Where $t$ is the thickness of the layer and $\sigma$ is the stress. When the stress is tensile the value is positive while a compressive stress has a negative value. The thickness of the layers is chosen in such a way that the mean stress is almost zero but still on the tensile side, thus a positive value. The thickness of the $\mathrm{SiO}_{2}$ and $\mathrm{Si}_{3} \mathrm{~N}_{4}$ is chosen to be $430 \mathrm{~nm}$ and $120 \mathrm{~nm}$, respectively. The fabrication scheme of the single side compensated membrane is shown in Table 2-III. The fabrication of the single side compensated valve is as follows. 


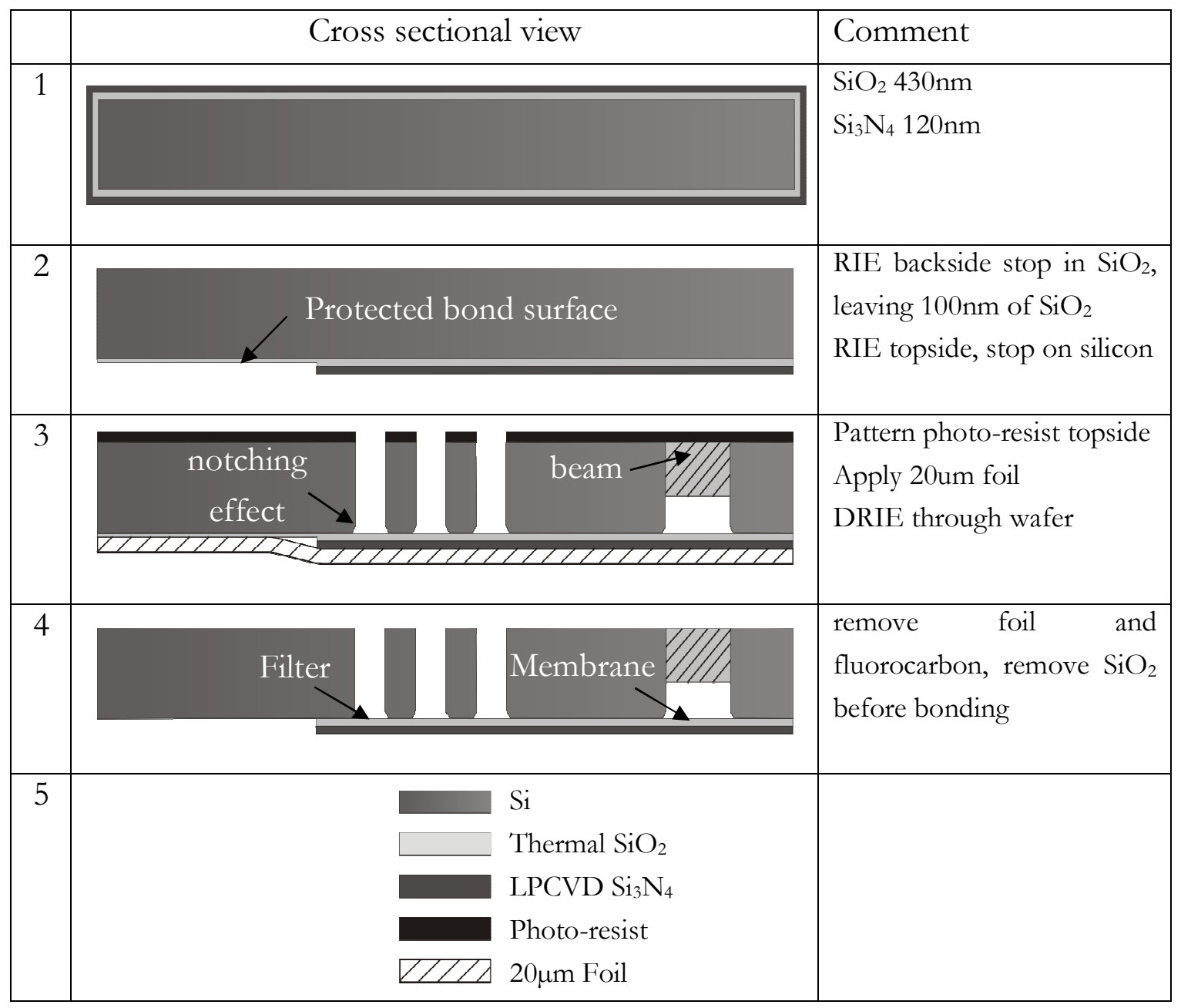

Table 5-IV: Single side compensated membrane fabrication scheme.

1. First a layer of $430 \mathrm{~nm} \mathrm{SiO}_{2}$ is thermally grown followed by the deposition $120 \mathrm{~nm} \mathrm{Si}_{3} \mathrm{~N}_{4}$ by an LPCVD process.

2. On the backside of the wafer these thin films are patterned by RIE defining the membrane and $2 \mu \mathrm{m}$ filter. $100 \mathrm{~nm}$ of the $\mathrm{SiO}_{2}$ layer is left to protect the bonding surface. The $\mathrm{Si}_{3} \mathrm{~N}_{4}$ and $\mathrm{SiO}_{2}$ layer on the topside are stripped by RIE.

3. On the topside of the wafer a photo-resist layer is applied which acts as a mask for the DRIE step. Before etching through the wafer a $20 \mu \mathrm{m}$ foil is applied to the backside of the wafer to avoid Helium leakage as discussed previously. The wafer is etched through and a free hanging membrane is obtained due to the notching effect. 
4. The wafers are cleaned in $\mathrm{HNO}_{3}$ and the fluorocarbon residues are burned in an oxidation step at $800^{\circ} \mathrm{C}$. Just before bonding the last $100 \mathrm{~nm}$ protecting $\mathrm{SiO}_{2}$ is removed from the bonding surface.

The bonding step is done by annealing at $1100^{\circ} \mathrm{C}$ in a nitrogen atmosphere. Before this step the membranes are tensile; no buckling is seen. It was observed that, after the high temperature step, the membranes were buckled, meaning they are under compressive stress. This means the stress is changing during annealing at $1100^{\circ} \mathrm{C}$. To get an idea of the mechanisms that are in play regarding the stress change, we take a closer look at the different materials. First, we will discuss the cause of stress in $\mathrm{SiO}_{2}$ and $\mathrm{Si}_{3} \mathrm{~N}_{4}$ layer just after deposition. Then we look at what happens when they are subjected to high temperature.

Two types of stress are considered; thermal stress and intrinsic stress. Thermal stress is caused by the difference in thermal expansion between two materials. Intrinsic stress is produced during the growth of a film. Several mechanisms can cause intrinsic stress, as there are: the incorporation of atoms, lattice mismatch, dislocations in the silicon lattice, grain boundary relaxation, chemical reaction, phase transformations and plastic deformation.

There are three materials that play a role in our system, these are; the silicon substrate, the $\mathrm{SiO}_{2}$ layer and the $\mathrm{Si}_{3} \mathrm{~N}_{4}$ layer. It is assumed that the silicon substrate will not plastically deform; it is the reference bulk material for determining the stress. In appendix D it is shown that this is not true under certain circumstances. For thermal oxide, grown on a silicon substrate at $1150^{\circ} \mathrm{C}$, a compressive stress of $0.29 \mathrm{GPa}$ is measured at room temperature; for the used procedure see appendix D. The stress is mainly caused by the difference in thermal expansion between the $\mathrm{SiO}_{2}$ and the silicon, thus thermal stress. After consecutive annealing for 1 hour at $1100^{\circ} \mathrm{C}$ no changes in stress could be measured. For this we found no exception. A lower stress value for the $\mathrm{SiO}_{2}$ layer was only found when it was annealed at $800^{\circ} \mathrm{C}$ for 59 hours. A stress of $0.26 \mathrm{GPa}$ is then measured. This is explained by the effect that the $\mathrm{SiO}_{2}$ film is slowly rearranging its structure and releasing stress to converge to a stress free situation at $800^{\circ} \mathrm{C}$. Since the stress is only caused by differences in thermal expansion, cooling down from $800^{\circ} \mathrm{C}$ to room temperature results in a lower stress than when it is cooled down from $1100^{\circ} \mathrm{C}$. Indeed, when the silicon wafer with the $0.26 \mathrm{GPa}$ compressive stressed $\mathrm{SiO}_{2}$ layer is again annealed at $1100^{\circ} \mathrm{C}$ the stress returns to its original value. 
The stress of $\mathrm{Si}_{3} \mathrm{~N}_{4}$ deposited on a silicon substrate by LPCVD at $850^{\circ} \mathrm{C}$ is measured to be $1.1 \mathrm{GPa}$ tensile. The mechanisms that are responsible for the intrinsic stress in $\mathrm{Si}_{3} \mathrm{~N}_{4}$ include dislocations in the silicon lattice, incorporation of atoms, lattice mismatch between the silicon surface and $\mathrm{Si}_{3} \mathrm{~N}_{4}$ film $[30,31]$. It has been shown that the stress at the deposition temperature - which is the inherent intrinsic stress only - is 15\% higher than the stress at room temperature [30]. This indicates that the thermal stress in the $\mathrm{Si}_{-} \mathrm{Si}_{3} \mathrm{~N}_{4}$ system is compressive as the total tensile stress is less when cooled down to room temperature. When the $\mathrm{Si}_{-} \mathrm{Si}_{3} \mathrm{~N}_{4}$ system is annealed at $1100^{\circ} \mathrm{C}$ for 1 hour it shows a minor change in stress towards $1 \mathrm{GPa}$. However, when annealing the $\mathrm{Si}-\mathrm{SiO}_{2}-\mathrm{Si}_{3} \mathrm{~N}_{4}$ system at $1100^{\circ} \mathrm{C}$ there seems to be a significant change. At this high temperature the viscosity of the $\mathrm{SiO}_{2}$ film decreases significantly. It is postulated that the $\mathrm{Si}_{3} \mathrm{~N}_{4}$ film is floating on the $\mathrm{SiO}_{2}$ layer at $1100^{\circ} \mathrm{C}$ and can release part of its intrinsic stress. To clarify; when an island of $\mathrm{Si}_{3} \mathrm{~N}_{4}$ is floating on a sticky sea of $\mathrm{SiO}_{2}$, the $\mathrm{Si}_{3} \mathrm{~N}_{4}$ can expand or contract without constraint. However, when the $\mathrm{Si}_{3} \mathrm{~N}_{4}$ film on top of the $\mathrm{SiO}_{2}$ is mechanically constraint in its movement, the stress release is slowed down. Some initial experiments that support this idea are presented in appendix D.

Now, we return to the single side compensated membrane which was mend to leave minor tensile stress but turned out to be compressive after annealing (fusion bonding). These membranes are $\sim 1.3 \mathrm{~mm}$ in diameter and are part of a larger island of $\mathrm{Si}_{3} \mathrm{~N}_{4}$ as is depicted in Figure 5-28. This island of $\mathrm{Si}_{3} \mathrm{~N}_{4}$ has a diameter of $6.3 \mathrm{~mm}$ and extends over the spiral spring. For a rough estimation of the stress in the $\mathrm{Si}_{3} \mathrm{~N}_{4}$ after annealing we did the following. The buckled membranes were etched in 1\% $\mathrm{HF}$ to reduce the thickness of the $\mathrm{SiO}_{2}$. It was found that the membranes become tensile when there is $260 \mathrm{~nm}( \pm 40 \mathrm{~nm})$ of $\mathrm{SiO}_{2}$ left. It must be said that the $\mathrm{SiO}_{2}$ thickness of the membrane could not be verified and this is an approximate value obtained by timing the etch step. When assuming that the stress in the thermal $\mathrm{SiO}_{2}$ is not changing, the tensile stress in the $\mathrm{Si}_{3} \mathrm{~N}_{4}$ can be calculated by:

$$
\begin{gathered}
\sigma_{S_{3} N_{4}} \cdot t_{S i_{3} N_{4}}+\sigma_{S i O_{2}} \cdot t_{S i O_{2}} \approx 0 \\
\sigma_{S i_{3} N_{4}}=\frac{-\sigma_{S i O_{2}} \cdot t_{S i O_{2}}}{t_{S i_{3} N_{4}}}=\frac{0.29 \cdot 260( \pm 40)}{120}=0.6( \pm 0.1) \mathrm{GPa}
\end{gathered}
$$


Thus the stress in the $\mathrm{Si}_{3} \mathrm{~N}_{4}$ seems to be changing from $1.1 \mathrm{GPa}$ to about $0.6 \mathrm{GPa}$ during annealing at $1100^{\circ} \mathrm{C}$ for 1 hour.

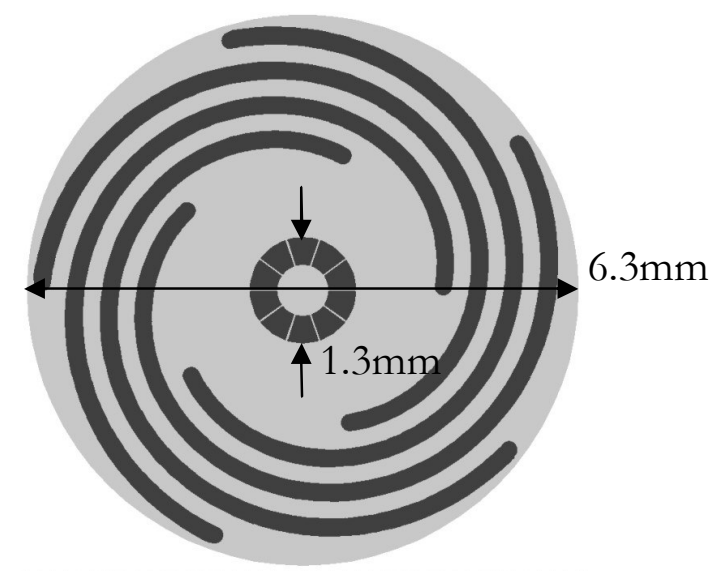

Figure 5-28: Dimensions of membrane as part of a larger $\mathrm{Si}_{3} \mathrm{~N}_{4}$ island.

Besides the changing stress value, another problem is observed with these membranes. They seem to be quite fragile and some break during cleaning and spin drying. Figure 5-29.a shows a broken valve membrane and Figure 5-29.b shows a broken filter membrane. In the close up of the broken filter - Figure 5-29.c - the $2 \mu \mathrm{m}$ holes can be distinguished. One can see that the membranes are curled. This is caused by the stress inside the bimorph membrane. The mean stress might be compensated but when they break the $\mathrm{Si}_{3} \mathrm{~N}_{4}$ wants to contract while the $\mathrm{SiO}_{2}$ is trying to expand resulting in the curling of the membrane. When a membrane is ruptured at a certain place it curls and rips itself loose till it cannot go further anymore as is seen in Figure 5-29.b. These curled membranes can have a height of $80 \mu \mathrm{m}$ which inhibits the bonding of the wafers. Thus, a single broken membrane inhibits the bonding of the devices which are still in tact.

Rupture of the valve membrane which is supposed to seal the valve seats was rare. However, rupture of the filter membrane which is positioned underneath the spiral spring is more often seen. This might be caused by the fact that when the spiral spring is bending it also rotates a little bit. Thereby, the filter membrane is twisted which might cause them to break. 


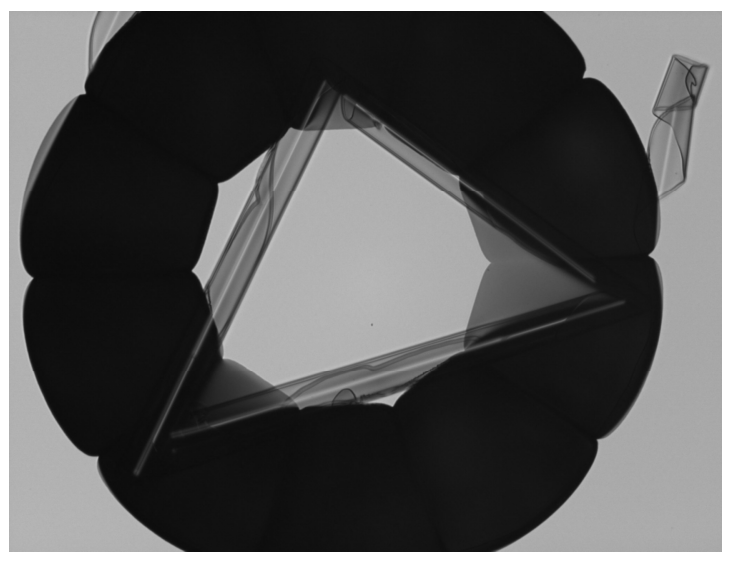

a)

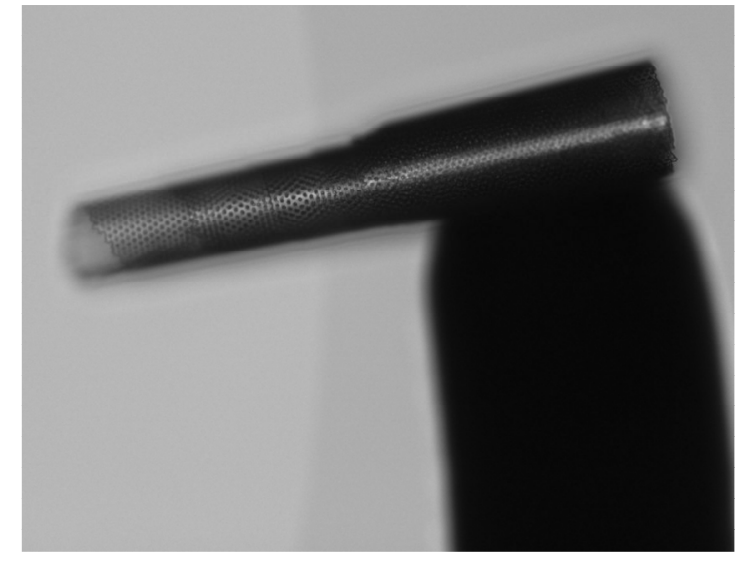

b)

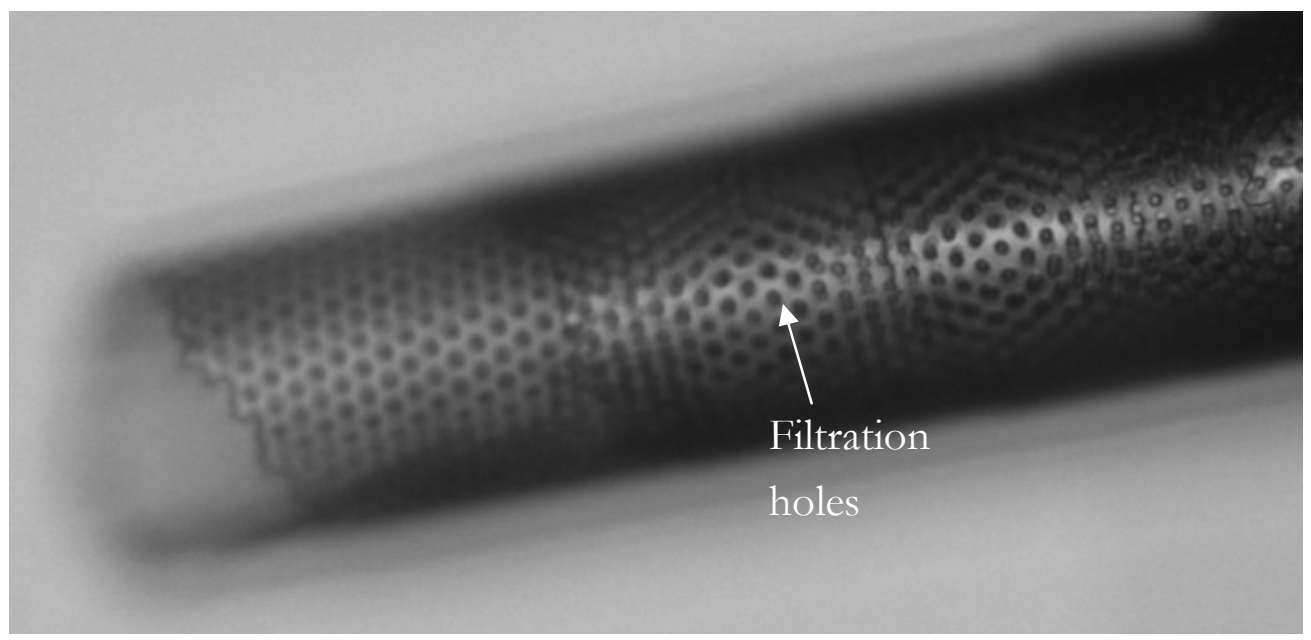

c)

Figure 5-29: a) Broken membrane b) Broken filter membrane c) Zoom in on the curved filter

The curling of a broken membrane can be solved by making a so called double compensated membrane; it is a membrane consisting of a $\mathrm{Si}_{3} \mathrm{~N}_{4}-\mathrm{SiO}_{2}-\mathrm{Si}_{3} \mathrm{~N}_{4}$ stack. On both sides of the compressive $\mathrm{SiO}_{2}$ layer the same thickness of tensile $\mathrm{Si}_{3} \mathrm{~N}_{4}$ is deposited. The membrane will not curl because there is no preference to bend upwards or downwards. We expect that such membranes will be stronger than the single side compensated membranes - at least a broken membrane will not inhibit the bonding of other devices due to curling - and this will be the subject of the next section. 
- Double side compensated membrane

The double side compensated membrane consists of a $\mathrm{Si}_{3} \mathrm{~N}_{4}-\mathrm{SiO}_{2}-\mathrm{Si}_{3} \mathrm{~N}_{4}$ stack. Two approaches are considered to make a tensile membrane. It is possible to slow down the release of stress in the $\mathrm{Si}_{3} \mathrm{~N}_{4}$ layer by constraining it mechanically. Or, one takes into account the change in stress, and tunes the thickness of the $\mathrm{SiO}_{2}$ and $\mathrm{Si}_{3} \mathrm{~N}_{4}$ layers in such a way that it is still tensile after annealing.

For the first fabrication method we make freely floating islands of $\mathrm{Si}_{3} \mathrm{~N}_{4}$ on top of $\mathrm{SiO}_{2}$. We assume that the stress in the $\mathrm{Si}_{3} \mathrm{~N}_{4}$ layer is $0.6 \mathrm{GPa}$ after bonding, more precisely after annealing at $1100^{\circ} \mathrm{C}$ for 1 hour. A total thickness of $120 \mathrm{~nm}$ is chosen for the $\mathrm{Si}_{3} \mathrm{~N}_{4}$ layers. With a $\mathrm{SiO}_{2}$ layer of $245 \mathrm{~nm}$ the membrane has $3 \mathrm{MPa}$ tensile stress as calculated below.

$$
\begin{aligned}
& \frac{\sigma_{S_{3} N_{4}} \cdot t_{S i_{3} N_{4}}+\sigma_{S i O_{2}} \cdot t_{S i O_{2}}}{t_{S i_{3} N_{4}}+t_{S i O_{2}}} \\
& =\frac{0.6 \cdot 120-0.29 \cdot 245}{365}=3 \mathrm{MPa}
\end{aligned}
$$

For this fabrication method a $\mathrm{SiO}_{2}$ layer is required on top of a $\mathrm{Si}_{3} \mathrm{~N}_{4}$ layer. Two options are considered to obtain a deposited $\mathrm{SiO}_{2}$ layer with a compressive stress of $0.3 \mathrm{GPa}$. The first method is to deposit poly silicon and give it a thermal oxidation treatment. Disadvantage of this method is the higher roughness of deposited poly silicon. Since roughness will cause leakage we chose for another method which results in lower roughness. For this method a TEOS layer is deposited by chemical vapor deposition (CVD). At room temperature, just after deposition, this layer has $10 \mathrm{MPa}$ tensile stress. For the membranes, we are not interested in tensile $\mathrm{SiO}_{2}$ but require a compressive stressed film. Therefore, the TEOS layer is annealed for 1 hour at $1100^{\circ} \mathrm{C}$ and changes into thermal oxide. As a result of the annealing, the TEOS layer gets approximately 3.5\% thinner due to out gassing and rearranging of the atoms. For annealed TEOS, a compressive stress of $0.29 \mathrm{GPa}$ is measured. For more details see appendix D and E.

The fabrication scheme of these membranes is shown in Table $5-\mathrm{V}$. The focus is only on the membrane that is supposed to seal the valve seats. Therefore, the spiral spring is left out. The fabrication process is as follows. 
1) On a $<110>380 \mu \mathrm{m}$ thick silicon wafer a six layer stack is applied. The first layer is a thermally grown $160 \mathrm{~nm}$ thick $\mathrm{SiO}_{2}$ layer and has multiple functions. First of all it is the protection layer for the silicon bonding surface. Second, it is used as a stop layer for several etch steps as shown later on. The next three layers are part of the membrane; it is the $\mathrm{Si}_{3} \mathrm{~N}_{4}-\mathrm{SiO}_{2}-\mathrm{Si}_{3} \mathrm{~N}_{4}$ stack. Both $\mathrm{Si}_{3} \mathrm{~N}_{4}$ layers are $60 \mathrm{~nm}$ thick and deposited by LPCVD at $850^{\circ} \mathrm{C}$. In between the $\mathrm{Si}_{3} \mathrm{~N}_{4}$ layers a $\mathrm{SiO}_{2}$ layer is present to compensate for the tensile stress in the $\mathrm{Si}_{3} \mathrm{~N}_{4}$. Therefore we use an annealed TEOS layer which has $0.29 \mathrm{GPa}$ compressive stress. The thickness of this layer is $245 \mathrm{~nm}$. On top of the $\mathrm{Si}_{3} \mathrm{~N}_{4}-\mathrm{SiO}_{2}-\mathrm{Si}_{3} \mathrm{~N}_{4}$ stack a $100 \mathrm{~nm}$ thick layer of poly silicon is deposited and approximately $10 \mathrm{~nm}$ of this layer is oxidized.

2) The poly silicon oxide is patterned on the backside to define the membrane.

3) Then a couple of wet etch steps are done. First the poly silicon layer is etched in a TMAH solution with the thin $10 \mathrm{~nm}$ oxide layer as mask. The $\mathrm{Si}_{3} \mathrm{~N}_{4}$ is etched in a $\mathrm{H}_{3} \mathrm{PO}_{4}$ solution followed by $\mathrm{SiO}_{2}$ etching in $1 \% \mathrm{HF}$. Finally, the second $\mathrm{Si}_{3} \mathrm{~N}_{4}$ layer is etched, again in $\mathrm{H}_{3} \mathrm{PO}_{4}$, and the underlying $\mathrm{SiO}_{2}$ layer prevents further etching. The typical isotropic shape of wet etched layers is depicted.

4) The poly silicon mask layer is stripped in TMAH and a photo-resist layer is spin-coated and patterned on the topside of the wafer. This photo-resist layer is used as mask for the DRIE step. The backside is protected with another photoresist layer.

5) The wafer is etched through by DRIE. Then the photo-resist is removed in $\mathrm{HNO}_{3}$. The wafer is cleaned and the fluorocarbon residue is burned at $800^{\circ} \mathrm{C}$. Just before bonding the protective $\mathrm{SiO}_{2}$ layer is stripped in $1 \% \mathrm{HF}$ and any last residue is lifting off. In this step also the $\mathrm{SiO}_{2}$ on the topside of the membrane is removed leaving only the $\mathrm{Si}_{3} \mathrm{~N}_{4}-\mathrm{SiO}_{2}-\mathrm{Si}_{3} \mathrm{~N}_{4}$ stack.

The fabricated membranes are annealed for 1 hour at $1100^{\circ} \mathrm{C}$. The $\mathrm{Si}_{3} \mathrm{~N}_{4}$ can now release its stress. We chose the thickness of the layers in such a way that the membrane should still be tensile after annealing and assumed a tensile stress of $0.6 \mathrm{GPa}$ in the $\mathrm{Si}_{3} \mathrm{~N}_{4}$ after 1 hour annealing at $1100^{\circ} \mathrm{C}$. However, after annealing the membranes still buckle, as was the case for the single side compensated membranes. 


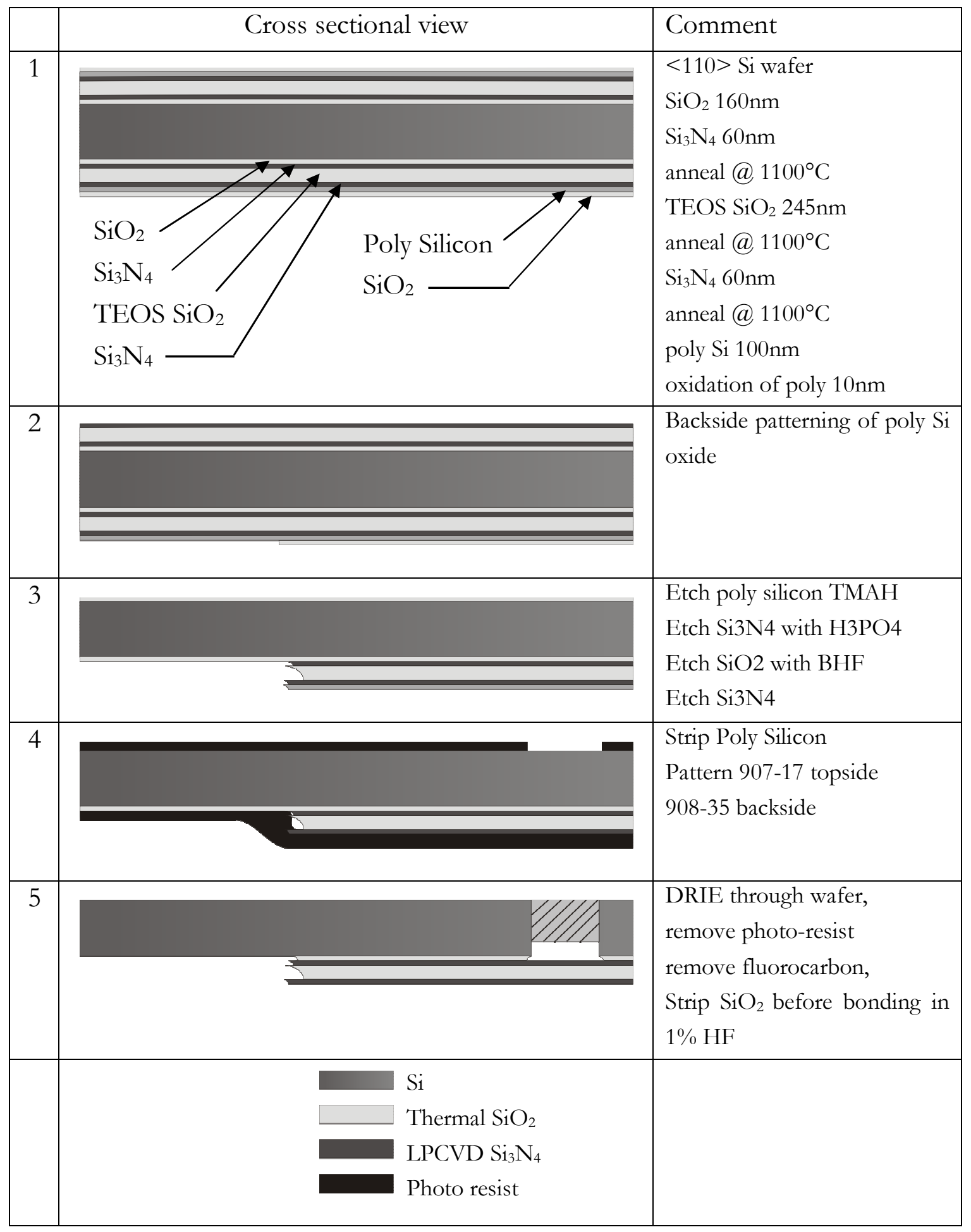

Table 5-V: Double side compensated membrane fabrication scheme 1.

Figure 5-30.a and .b show the membrane before and after annealing, respectively. One can see the typical flower shape due to the notching effect. The membrane in Figure 5-30.a is not buckled while the annealed membrane in Figure 5-30.b clearly 
shows wrinkles. To find out the approximate stress in the $\mathrm{Si}_{3} \mathrm{~N}_{4}$ after annealing, an additional layer of $44 \mathrm{~nm} 1.1 \mathrm{GPa}$ tensile $\mathrm{Si}_{3} \mathrm{~N}_{4}$ is deposited. The membrane is now tensile again. The situation is shown in Figure 5-31. By etching the $\mathrm{Si}_{3} \mathrm{~N}_{4}$ back with $50 \% \mathrm{HF}$ in steps of about $10 \mathrm{~nm}$ we observed that the membrane is still tensile with $21 \mathrm{~nm}$ of $\mathrm{Si}_{3} \mathrm{~N}_{4}$ on both sides of the membrane. The membrane is buckled when there is $10.7 \mathrm{~nm} \mathrm{Si} \mathrm{N}_{4}$ left. Thus, between these values $-16 \mathrm{~nm}( \pm 5 \mathrm{~nm})-$ the membrane changes from tensile to compressive. From this we calculate the stress in the annealed $\mathrm{Si}_{3} \mathrm{~N}_{4}$ layer.

$$
\begin{gathered}
\sigma_{S i_{3} N_{4}} \cdot t_{S i_{3} N_{4}}+\sigma_{S i_{3} N_{4(1.1 G P a)}} \cdot t_{S i_{3} N_{4(1.1 G P a)}}+\sigma_{S i O_{2}} \cdot t_{S i O_{2}} \approx 0 \\
\sigma_{S i_{3} N_{4}}=\frac{-\sigma_{S i_{3} N_{4(1.1 G P a)}} \cdot t_{S i_{3} N_{4(1.1 G P a)}}+\sigma_{S i O_{2}} \cdot t_{S i O_{2}}}{t_{S i_{3} N_{4}}} \\
=\frac{-1.1 \cdot[2 \cdot 16( \pm 5)]+0.3 \cdot 245}{2 \cdot 60}=0.32( \pm 0.1) \mathrm{GPa}
\end{gathered}
$$

The stress in the $\mathrm{Si}_{3} \mathrm{~N}_{4}$ layer of the single side compensated membranes was found to be $0.6( \pm 0.1) \mathrm{GPa}$ tensile. The stress in these double side compensated membranes is even lower, $0.32( \pm 0.1) \mathrm{GPa}$ tensile. The single side compensated membranes are part of a $6.3 \mathrm{~mm} \mathrm{Si}_{3} \mathrm{~N}_{4}$ island while these double side compensated membranes are $1.5 \mathrm{~mm}$ in diameter. The $\mathrm{Si}_{3} \mathrm{~N}_{4}$ has been annealed at $1100^{\circ} \mathrm{C}$ for several hours which is now believed to release a substantial part of the intrinsic stress. Moreover, we think that the stress in smaller islands is released faster. Appendix D gives more evidence in this direction.

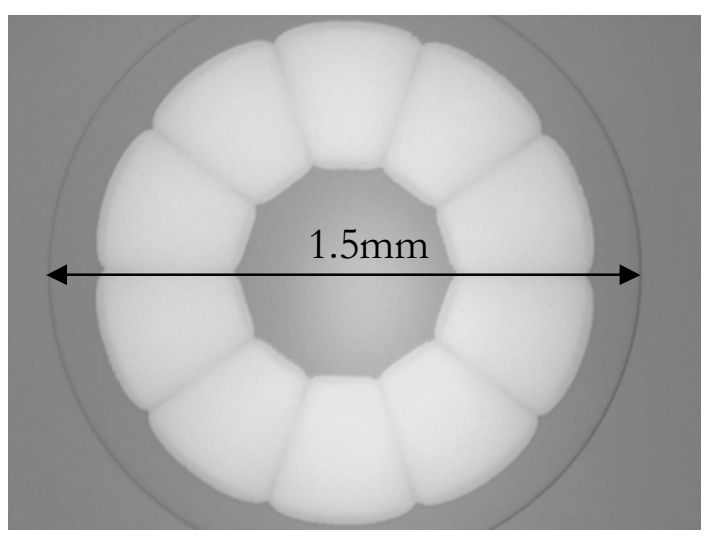

a)

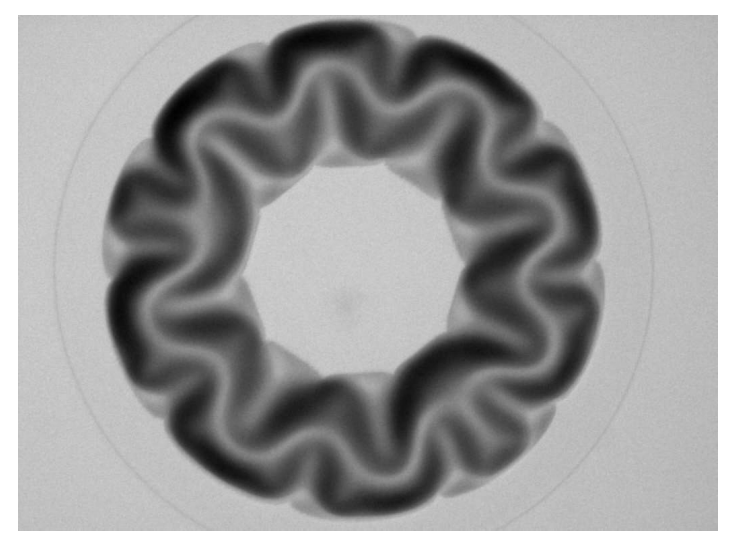

b)

Figure 5-30: a) Flower membrane before anneal b) Flower membrane after 1 hour at $1100^{\circ} \mathrm{C}$ 


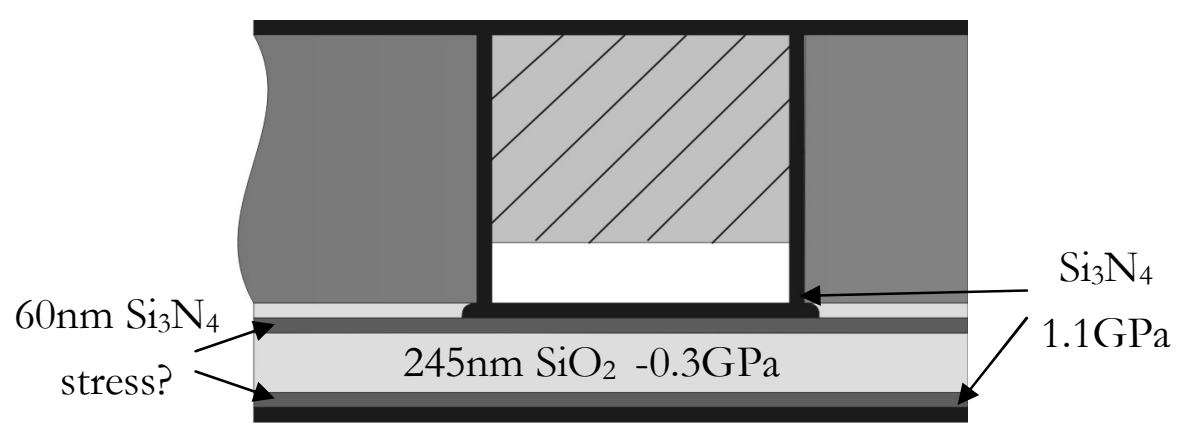

Figure 5-31: Membrane covert with an additional layer of $\mathrm{Si}_{3} \mathrm{~N}_{4}$

The second fabrication approach for the double side compensated membranes is shown in Table 5-VI. With this fabrication method the $\mathrm{Si}_{3} \mathrm{~N}_{4}$ film is mechanically constraint and we expect that the stress is released much slower. In appendix D some experiments are presented which support this assumption. We assume a stress in the $\mathrm{Si}_{3} \mathrm{~N}_{4}$ film of $1.0 \mathrm{GPa}$ after bonding (appendix D). The thickness of the $\mathrm{Si}_{3} \mathrm{~N}_{4}$ layers (total $160 \mathrm{~nm}$ ) and $\mathrm{SiO}_{2}$ layer $(477 \mathrm{~nm})$ is tuned in such a way that the mean stress is tensile.

$$
\begin{aligned}
& \frac{\sigma_{S_{3} N_{4}} \cdot t_{S i_{3} N_{4}}+\sigma_{S i O_{2}} \cdot t_{S i O_{2}}}{t_{S i_{3} N_{4}}+t_{S i O_{2}}} \\
& =\frac{1.0 \cdot 160-0.29 \cdot 477}{637}=34 \mathrm{MPa}
\end{aligned}
$$

The fabrication starts with the shaping of some anchor points for the final $\mathrm{Si}_{3} \mathrm{~N}_{4}-$ $\mathrm{SiO}_{2}-\mathrm{Si}_{3} \mathrm{~N}_{4}$ membrane. This is the mechanical constraint which ensures that the $\mathrm{Si}_{3} \mathrm{~N}_{4}$ cannot release its stress at high temperature.

1) On the backside of a $<110>380 \mu \mathrm{m}$ thick silicon wafer a photo-resist layer is patterned to determine the mechanical anchor. The silicon is etched by DRIE for a depth of $20 \mu \mathrm{m}$ and the fluorocarbon residue is removed.

2) Then, the wafer is covered with a $495 \mathrm{~nm}$ thick TEOS layer. This layer is not annealed and thus the stress is close to zero.

3) On the backside of the wafer a layer of photo-resist is spin-coated and the topside TEOS layer is removed in $1 \% \mathrm{HF}$.

4) Subsequently, a layer of photo-resist is spin-coated on the topside and patterned to define the membrane. The wafer is etched through by DRIE and we selectively stop on the TEOS layer. 


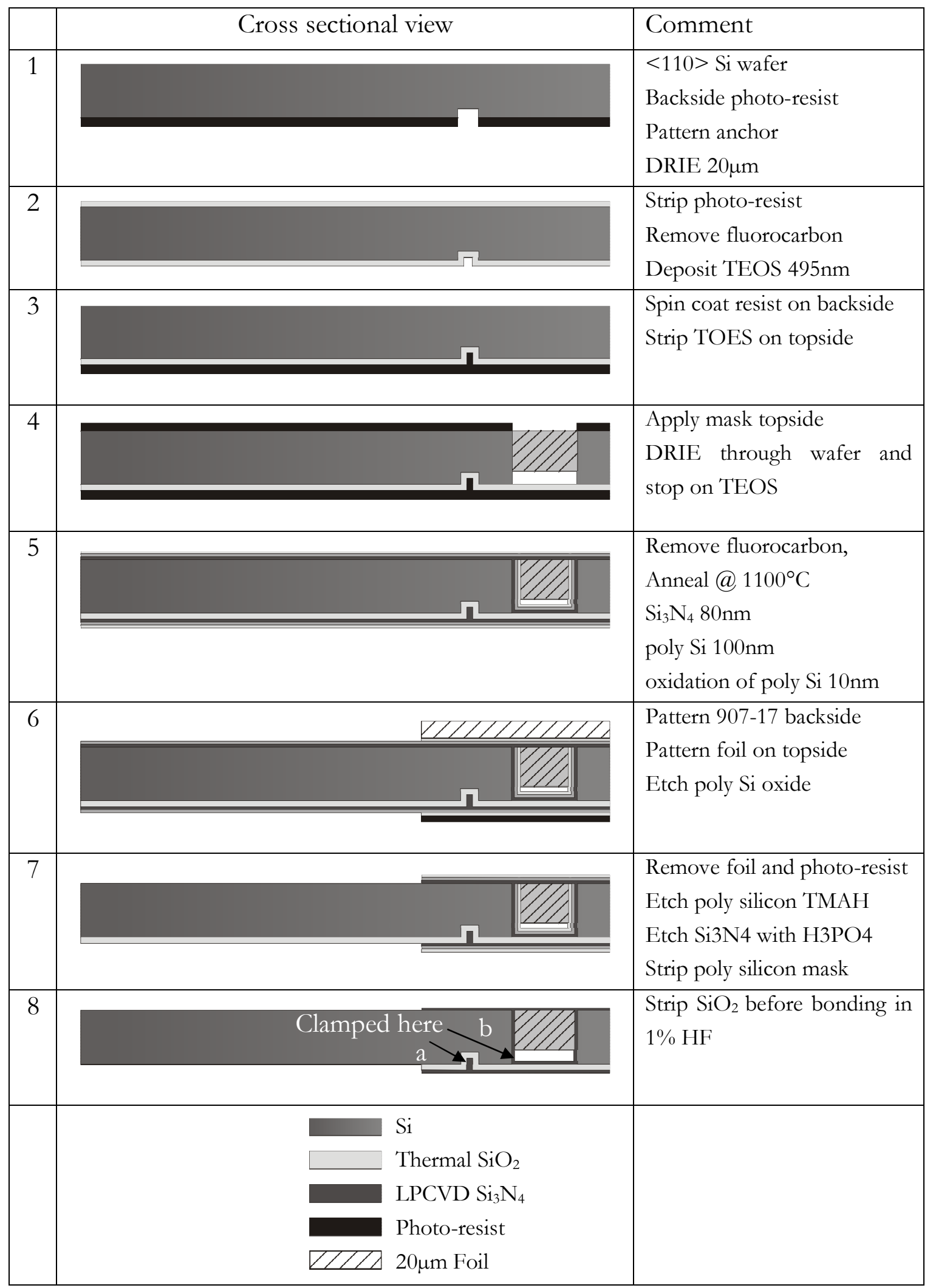

Table 5-VI: Double side compensated membrane fabrication scheme 1. 
5) After this etch step the fluorocarbon residue is removed as discussed in section 5.4.1. Since we need a $\mathrm{SiO}_{2}$ layer with a compressive stress of $0.3 \mathrm{GPa}$ the TEOS is annealed at $1100^{\circ} \mathrm{C}$ for one hour. The layer thickness reduced to $477 \mathrm{~nm}$ due to the annealing. When the wafer is taken out of the oven and cooled down to room temperature, the membranes buckle due to the compressive stress. This makes them quite fragile and therefore it is important to deposit the $\mathrm{Si}_{3} \mathrm{~N}_{4}$ directly after this step. The deposition of the $80 \mathrm{~nm}$ thick $\mathrm{Si}_{3} \mathrm{~N}_{4}$ - on both sides of the membrane - is done at $850^{\circ} \mathrm{C}$. At the start of this process - when the wafers are heated to this temperature - the membranes become tensile again. This is due to the difference in thermal expansion between the $\mathrm{SiO}_{2}$ and silicon substrate. Thus the membranes are not buckled during the deposition process of the $\mathrm{Si}_{3} \mathrm{~N}_{4}$. After the $\mathrm{Si}_{3} \mathrm{~N}_{4}$ deposition, a $100 \mathrm{~nm}$ thick layer of poly silicon is deposited and $10 \mathrm{~nm}$ of this layer is oxidized.

6) The poly silicon oxide is patterned. The backside of the wafer can simply be patterned by using a spin-coated photo-resist mask. The topside of the membrane needs to be protected for the next wet etch step. To do this a $20 \mu \mathrm{m}$ thick foil is laminated on top of the wafer and patterned. Thereby the cavity above the membrane is sealed and the membrane is protected. The poly silicon oxide is etched in $1 \% \mathrm{HF}$.

7) The foil and photo-resist are removed by $\mathrm{HNO}_{3}$. The poly silicon is etched in TMAH and thereafter the $\mathrm{Si}_{3} \mathrm{~N}_{4}$ is etched in $\mathrm{H}_{3} \mathrm{PO}_{4}$.

8) Before bonding the poly silicon mask layer is removed in TMAH and the $\mathrm{SiO}_{2}$ is stripped.

The fabricated membranes are annealed at $1100^{\circ} \mathrm{C}$ for 1 hour. Since the $\mathrm{Si}_{3} \mathrm{~N}_{4}$ is clamped - as indicated in Table 5-VI.8 - the stress in this layer is not changing much. This fabrication process shows two examples of how the $\mathrm{Si}_{3} \mathrm{~N}_{4}$ can be clamped.

a) The first example shows how the $\mathrm{Si}_{3} \mathrm{~N}_{4}$ is constraint by geometry. By etching a trench in the silicon substrate - as depicted in step 1 - and depositing the layers inside the trench, the $\mathrm{Si}_{3} \mathrm{~N}_{4}$ layer is anchored in the silicon.

b) The second method to constrain the $\mathrm{Si}_{3} \mathrm{~N}_{4}$ is simply by depositing it on the silicon substrate. The $\mathrm{Si}_{3} \mathrm{~N}_{4}$ is deposited inside the cavity above the membrane. It is attached to the silicon sidewall of the etched cavity. 
The membranes that are fabricated by this process are indeed tensile as shown in Figure 5-32.a, meaning the $\mathrm{Si}_{3} \mathrm{~N}_{4}$ stress is larger than $1.0 \mathrm{GPa}$ as calculated in equation (5.19). Figure 5-32.b shows a ruptured double side compensated membrane. This membrane is not curled - like the single side compensated membrane (Figure 5-29.a) - as is expected.

Both fabrication methods have been tested to make double side compensated membranes. When the $\mathrm{Si}_{3} \mathrm{~N}_{4}$ is clamped, the stress release is minor, as is demonstrated by the second fabrication method. Care must be taken with the stress in the $\mathrm{Si}_{3} \mathrm{~N}_{4}$ when it is free to float on a slippery layer as is the case with the first fabrication method. Part of the tensile stress is then released. It is observed that for smaller free floating $\mathrm{Si}_{3} \mathrm{~N}_{4}$ islands the stress release seems to be faster. Although the membranes that were made with this fabrication method were buckled, we believe that the thickness of the layers can be tuned in such a way that they stay tensile.

When we compare the stress in the membranes of the two fabrication methods the following can be said. The stress in the constraint $\mathrm{Si}_{3} \mathrm{~N}_{4}$ is much higher than for the $\mathrm{Si}_{3} \mathrm{~N}_{4}$ layer which could release part of this stress. Although the mean stress can be compensated, the internal stress of the $\mathrm{Si}_{3} \mathrm{~N}_{4}-\mathrm{SiO}_{2}-\mathrm{Si}_{3} \mathrm{~N}_{4}$ membrane is much higher in the clamped case. The higher the internal stresses the more fragile the membrane becomes. Therefore we prefer the method of stress release of the $\mathrm{Si}_{3} \mathrm{~N}_{4}$ which results in lower internal stress in the membrane.

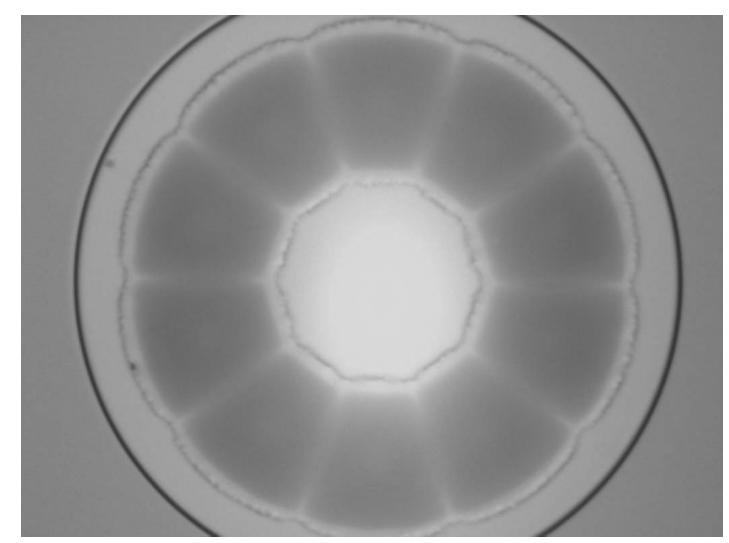

a)

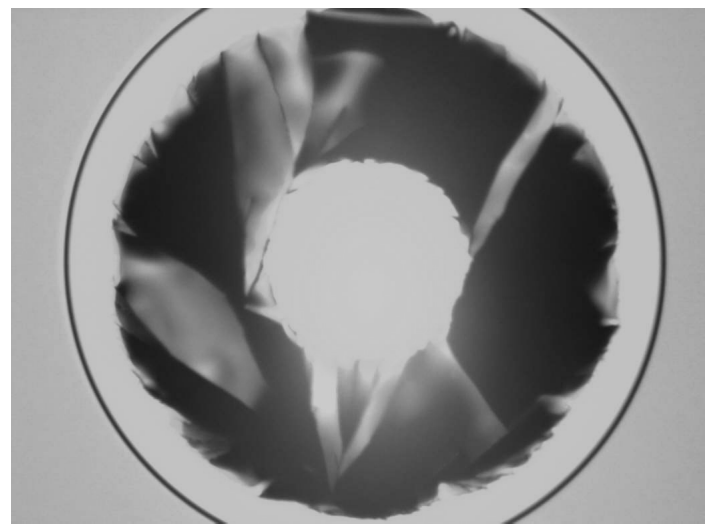

b)

Figure 5-32: a) Flower membrane after anneal b) Broken flower membrane after anneal 


\subsection{Conclusions}

In this chapter the research is described towards a leak-tight micro valve. Most micro valves make a seal between two hard surfaces. In this case two properties where found that can cause leakage, i.e. the surface waviness and trapped particles in the valve. Both cause a gap in between the valve seat and its sealing and thus a leak. The surface waviness is in the order of tens of nanometer which is enough to cause a leak which exceeds the allowed leak rate. Although filters can be integrated, there is always a chance that particles - already present in the system - get stuck inside the valve. For a valve with two hard surfaces an enormous amount of force is required to crush dirt particles and to deform the waviness of silicon surface to make a proper seal. Due to this high force an actuator is required which is very big compared to the size of the rest of the valve. When miniaturization is pursued this high force is a bottle neck. To allow for further miniaturization of micro valves especially leak-tight micro valves - a novel valve is presented which promises to be leak-tight without requiring a high force to close.

The new valve is designed in such a way that surface waviness and trapped particles will not cause a gap through which leakage is possible. A valve seat pattern is designed in such a way that the chance that multiple particles cause leakage is reduced. The shape of these valve seats encourages particles to slide towards a lower position in between the valve seats where they are neutralized. The valve seat pattern is sealed with a flexible thin film membrane composed of $\mathrm{SiO}_{2}$ and $\mathrm{Si}_{3} \mathrm{~N}_{4}$. These materials are not permeable for gases, which cannot be said for most flexible rubbers. This flexible membrane is pulled over the valve seat pattern and adjusts to the surface waviness. The force that is required to pull the membrane over the valve seat pattern is in the $\mathrm{mN}$ range which allows for a small actuator and thus further miniaturization.

The fabrication of this valve appeared to be quite complicated. The valve is consisting of two silicon wafers which are fusion bonded in the final step. The valve seat and chamber are shaped in the bottom wafer. The top wafer contains the spring mechanism and a thin film membrane which closes the valve. The valve seat fabrication is pretty straightforward although care must be taken with residues left by dry etching steps. These residues can inhibit the bonding process. It is therefore advised to use wet etch step there where possible. 
The fabrication of the thin film membrane consisting of $\mathrm{SiO}_{2}$ and $\mathrm{Si}_{3} \mathrm{~N}_{4}$ presents a yet unsolved problem. The stress in the $\mathrm{Si}_{3} \mathrm{~N}_{4}$ layer of these membranes can change during annealing at $1100^{\circ} \mathrm{C}$, which is a necessary step for the bonding of silicon wafers. When the $\mathrm{Si}_{3} \mathrm{~N}_{4}$ layer is clamped this process seems slow, but when $\mathrm{SiO}_{2}$ is underneath, the tensile stress releases faster. Due to the release of this stress the membranes buckle after annealing. Two fabrication methods have been presented to make double side compensated membranes. It is shown that we can make $\mathrm{Si}_{3} \mathrm{~N}_{4-}$ $\mathrm{SiO}_{2}-\mathrm{Si}_{3} \mathrm{~N}_{4}$ membranes which are still tensile after annealing. Integration of this process into the fabrication of the valve has not yet been done. So far, we have not been able to make a fully functioning valve. Thus, unfortunately we are not able to confirm the working principle of the sealing mechanism.

\subsection{References}

[1] Mueller J., A review and applicability assessment of MEMS-based microvalve technologies for microspacecraft propulsion, 35th ALAA/ASME/SAE/ASEE Joint Propulsion Conference and Exhibit, Los Angeles, California, USA (1999).

[2] Becker H. and G. C., Polymer microfabrication technologies for microfluidic systems, Analytical and Bioanalytical Chemistry 390 (2008), pp. 89-111.

[3] Browne V.d'A. and John J.E.A., Vacuum radial flow from the viscous through the free molecule regime, V acuum 20 (1970), pp. 525-533.

[4] Malik I.J., Pirooz S., Shive L.W., Davenport A.J. and Vitus C.M., Surface roughness of silicon wafers on different lateral length scales, J. of the Electrochemical Society 140 (1993), pp. L75-L77.

[5] Fazal I., Development of a gas microvalve based on fine- and micromachining, Tranceducer Science and Technology, University of Twente, Enschede (2007).

[6] Oh K.W. and Ahn C.H., A Review of Microvalves, J. of Micromechanics and Microengineering 16 (2006), pp. R13-R39.

[7] Choi J.-W, Oh K.W., Han A., et al., Development and characterization of microfluidic devices and systems for magnetic bead-based biochemical detection, Biomedical Microdevices 3 (2001), pp. 191-200. 
[8] Hirano M., Yanagisawa K., Kuwano H. and Nakano S., Microvalve with Ultra-Low Leakage, Proceedings of the IEEE Micro Electro Mechanical Systems (1997), pp. 323-326.

[9] Yang X., Hölke A., Jacobson S.A., Lang J.H., Schmidt M.A. and Umans S.D., An Electrostatic OnOff Microvalve Designed for Gas Fuel Delivery for the MIT Microengine, J. of Microelectromechanical Systems 13 (2004).

[10] Jerman H., Electrically activated, normally closed diaphragm valves, J. of Micromechanics and Microengineering 4 (1994), pp. 210-216.

[11] Rich C.A. and Wise K.D., A high-flow thermopneumatic microvalve with improved efficiency and integrated state sensing, J. of Microelectromechanical Systems 12 (2003).

[12] Yang X., Grosjean C. and Tai Y-C., Design, Fabrication, and Testing of Micromachined Silicone Rubber Membrane Valves, J. of Microelectromechanical Systems 8 (1999).

[13] G. J. Van Amerongen, The Permeability of Different Rubbers to Gases and Its Relation to Diffusivity and Solubility, Journal of Applied Physics 17 (1946), pp. $972-$ 985 .

[14] Rogge T., Rummler Z. and Schomburg W.K., Polymer micro valve with a hydraulic piezo-drive fabricated by the AMANDA, Sensors and Actuators, A: Physical 110 (2004), pp. 206-212.

[15] Köhler J., Bejhed J., Kratz H., et al., A hybrid cold gas microthruster system for spacecraft, Sensors and Actuators A: Physical 97-98 (2002), pp. 587-598.

[16] Yang E.H., Lee C., Mueller J. and George T., Leak tight piezoelectric microvalve for high pressure gas micropropulsion, J. Microelectromech. Syst. 13 (2004), pp. 799-807.

[17] Mueller J., Thruster Options for Microspacecraft: A Review and Evaluation of State-of-the-Art and Emerging Technologies, Micropropulsion for Small Spacecraft of the Progress in Astronautics and Aeronautics series 147 (2000), p. 45.

[18] http://www.20sim.com/. 
[19] Jansen H.V., de Boer M.J., Unnikrishnan S., Louwerse M.C. and Elwenspoek M.C., Black silicon method X: a review on high speed and selective plasma etching of silicon with profile control, J. Micromech. Microeng. 19 (2009).

[20] Jansen H.V., Verhagen R. and Elwenspoek M.C., The black silicon method III: design rules, modelling, optimisation and performance of precision position systems for scanning probe, gripping and other MEMS applications, Proceedings of Handling and Assembly of microparts (1994).

[21] Giovanni M. Di, Flat and Corrugated Diaphragm Design Handbook, Marcel Dekker Inc., New York (1982).

[22] Hwang G. and Giapis K.P., On the origin of the notching effect during etching in uniform high density plasmas, J V ac Sci Technol B 15 (1997).

[23] Müller B. and Stoffel A., Tensile strength characterization of lowtemperature fusion-bonded silicon wafers, J. Micromech. Microeng. 1 (1991), pp. 161 166.

[24] Gui C., Elwenspoek M.C., Tas N. and Gardeniers J.G.E., The effect of surface roughness on direct wafer bonding, J. of Applied Physics 85 (1999), pp. 74487454.

[25] Gui C., Oosterbroek R.E., Berenschot J. W., et al., Selective Wafer Bonding by Surface Roughness Control, J. of Electrochemical Society 148 (2001), pp. G225G228.

[26] Oosterbroek R.E., Modeling, Design and Realization of Microfluidic Components, Transducer Science and Technology, University Twente, Enschede (1999).

[27] Gui C., Albers H., Gardeniers J.G.E., Elwenspoek M. and Lambeck P.V., Fusion bonding of rough surfaces with polishing technique for silicon micromachining, Microsystem Technologies 3 (1997), pp. 122-128.

[28] Marcus R.B. and S. T.T., The oxidation of shaped silicon surfaces, J. of the Electrochemical Society (1982), pp. 1252-1260.

[29] Minha P.N., Ono T. and Esashi M., Nonuniform silicon oxidation and application for the fabrication of aperture for near-field scanning optical microscopy, Applied physics letters 75 (1999), pp. 4076-4078. 
[30] Tamura M. and Sunami H., Generation of Dislocations Induced by Chemical Vapor Deposited Si3N4 Films on Silicon, Japan. J. Appl. Phys. 12 (1972), pp. 1097-1105.

[31] Noskov A.G., Gorokhov E.B., Sokolova G.A., Trukhanow E.M. and Stenin S.I., Correlation Between Stress and Structure in Chemically Vapour Deposited Silicon Nitride Films, Thin Solid Films 163 (1988), pp. 129-143. 


\section{Conclusions}

This chapter gives a summary and résumés the conclusions of the previous chapters. 
To enable formation flying of micro satellites an extremely miniaturized propulsion system is required. Conventional solutions are too big and heavy. Micro system technology (MST) is shown to be an excellent technology to scale down several components of a propulsion system.

A miniaturized propulsion system is presented of a cold gas blow-down rocket engine. The functional design of this system is elaborated. The system consists of two parts, i.e. the low pressure storage system and the feeding and thruster system. The low pressure storage system ensures an operation pressure in between 1 and 4.5bar. The feeding and thruster system can generate the thrust required for position corrections of the satellite. The latter system consists of several functional modules. These modules are: an active valve, a particle filter, and a nozzle. For these modules it is shown that the characteristic dimensions are in the micrometer range and thus MST is a suitable technology. Furthermore, an electronic module is included to actuate the valve and to read out the pressure and temperature sensor.

A modular platform is presented to put these functional modules together. The modules are adjusted to fit in a baseline package. A simple glass tube is used as hermetically sealed package. It functions as a fluidic interconnection and a macro support for the functional modules. The modules can be tested and characterized before they are integrated in the glass tube package. The valve and nozzle module are fusion bonded onto the glass tube package. This system is tested up to 12bar which is significantly higher than the operation pressure - without mechanical failure. Other functional modules - like the electronic and filter module - are implemented by suspending them inside the glass tube by o-rings. The glass tube package, including all the modules, can be attached to the pressure storage tank, through which also the electrical connections are made.

The nozzle is used to increase the velocity of the exhaust and thereby the efficiency of the propulsion system is increased. Three fabrication methods are explored to make a truly 3D conical converging-diverging nozzle shape. These fabrication methods are deep reactive ion etching (DRIE), femtosecond laser machining (FLM) and a method which uses powder blasting and a heat treatment. The latter method 
is used to make a nozzle in glass while DRIE and FLM are used to shape a nozzle in silicon.

The DRIE fabrication method seemed promising because of the smooth sidewalls that can be obtained, which is an important requirement for the nozzle. We tried to make a nozzle shape by tuning the process settings to obtain a negative tapered profile. For the diverging part of the nozzle - which is the most important part this means a half angle in between 15 and 20 degrees. The largest negative taper that we could obtain had a half angle of 5 degrees. This angle is not large enough for the diverging part of the nozzle. Therefore, we were not able to make a proper nozzle with this method.

FLM is shown to be a good method to fabricate nozzles in silicon. The desired half angle could be tuned to the requirements. There are a couple of disadvantages regarding this method. First of all, a sharp corner is present in the throat of the nozzle which has a negative effect on the performance. Second, the roughness of the sidewall is in the order of microns. This increases the thickness of the fluid boundary and thereby the thrust is reduced. At last, this method requires double sided alignment for which our system was not optimized. Misalignment results in an asymmetrical nozzle shape.

The glass nozzles that are made with the method of powder blasting in combination with heat treatment have a smooth nozzle sidewall. This is most important in the nozzle throat. Although the powder blasting process results in a rough sidewall, this is corrected during the heat treatment. Due to reflow of the glass the roughness is reduced considerably. The radius of curvature and the position of the throat are controlled by the temperature step and can be adjusted according to specifications. A disadvantage of this method is the short inlet length, but the impact on the performance is minor. Furthermore, the powder blasting process is not very accurate and introduces deviations in the dimensions of the nozzle. Since the radius of curvature of the nozzle throat and the sidewall roughness are the most important parameters for the nozzle performance the powder blast method is preferred.

The valve module is most crucial for a successful satellite mission. The leak rate of the valve - by which the lifetime of the satellite is reduced - should be below $1.6 \cdot 10^{-4} \mathrm{sccm}$. This is a requirement which - in conventional valves - is only met by 
using a large force to close the valve. A large force implies a large actuator. When miniaturization is pursued, the approach of brute force is therefore not favorable. To allow for even smaller valves a novel design is presented requiring low force while still promising to be leak-tight.

Valve leakage is caused by waviness of two hard sealing surfaces and by trapped particles. The new valve is designed in such a way that the surface waviness is not causing any leakage. A flexible thin film membrane is pulled over a valve seat pattern to make a leak-tight seal. Due to the flexibility of this membrane it can deform and adjust to the surface topography. The force that is required to pull the membrane over the valve seat pattern is in the $\mathrm{mN}$ range. The chance that multiple particles induce leakage is reduces by a valve seat pattern consisting of several compartments. Furthermore, by making a triangular or rounded shaped valve seat, trapped particles are encouraged to slide towards a position in between the valve seats. In this position they do not contribute to leakage.

During the fabrication of the valve several technological challenges came forth. The valve consists of two wafers, i.e. the valve seat wafer and the membrane wafer. They are fabricated separately and finally fusion bonded. The success of this final process step is influenced by previous process steps. Residues left behind by dry etch steps are sometimes hard to remove and can inhibit the bonding process. Therefore it is advised to avoid dry etching - there where possible - and use wet etch step instead. Moreover, it is crucial to use a protective layer - like $\mathrm{SiO}_{2}-$ from the very start of processing until the pre-bonding step.

The fabrication of the thin film membrane consisting of $\mathrm{SiO}_{2}$ and $\mathrm{Si}_{3} \mathrm{~N}_{4}$ was found to be challenging. This membrane is used as a flexible thin film sealing. It is therefore important that the stress in this film is close to zero. However, it should absolutely not be compressive because then the membrane will buckle. It is found that annealing of these thin film membranes has a large influence on the stress. This anneal step is necessary for the final bonding step. The compressive stress in the $\mathrm{SiO}_{2}$ does not change. However, during a high temperature anneal the stress in $\mathrm{Si}_{3} \mathrm{~N}_{4}$ can change considerably in certain circumstances. When $\mathrm{Si}_{3} \mathrm{~N}_{4}$ - on top of a $\mathrm{SiO}_{2}$ layer - is annealed at $1100^{\circ} \mathrm{C}$ it tends to release its stress very fast in certain circumstances. The $\mathrm{SiO}_{2}$ layer is fluent at this high temperature. When the $\mathrm{Si}_{3} \mathrm{~N}_{4}$ on top of this fluent layer is not constraint in its movement it can contract or expand 
easily and thereby release stress. When the $\mathrm{Si}_{3} \mathrm{~N}_{4}$ layer is clamped the release of stress is slowed down considerably. The mechanisms that are responsible for the change in stress are not yet fully understood and require more experiments. Nevertheless, it is shown that we can make $\mathrm{Si}_{3} \mathrm{~N}_{4}-\mathrm{SiO}_{2}-\mathrm{Si}_{3} \mathrm{~N}_{4}$ membranes which are still tensile after annealing. These double side compensated membranes are not yet integrated in the fabrication of the valve. Therefore, we have not yet been able to show that this novel sealing mechanism is leak-tight.

To be able to integrate the tensile double side compensated membranes in the valve further research is required. There seems to be a relation between the diameter of a free floating $\mathrm{Si}_{3} \mathrm{~N}_{4}$ island and the speed at which it releases stress. Experiments are required to find this relation. Although less crucial, the valve seats can be further optimized to reduce the chance that a particle will cause leakage. Therefore, a fabrication scheme is presented to make round shaped valve seats. 



\section{Appendix A - Electronics module}

Figure A-I shows the electronic circuit including microcontroller. The circuit converts a $3.3-5 \mathrm{~V}$ supply voltage into a $180 \mathrm{~V}$ source to actuate the piezo-disc. In Table A-I a list of electronic components is given. Table A-II shows the C-program for the ATtiny 24 microcontroller. At last some voltage measurements are given.

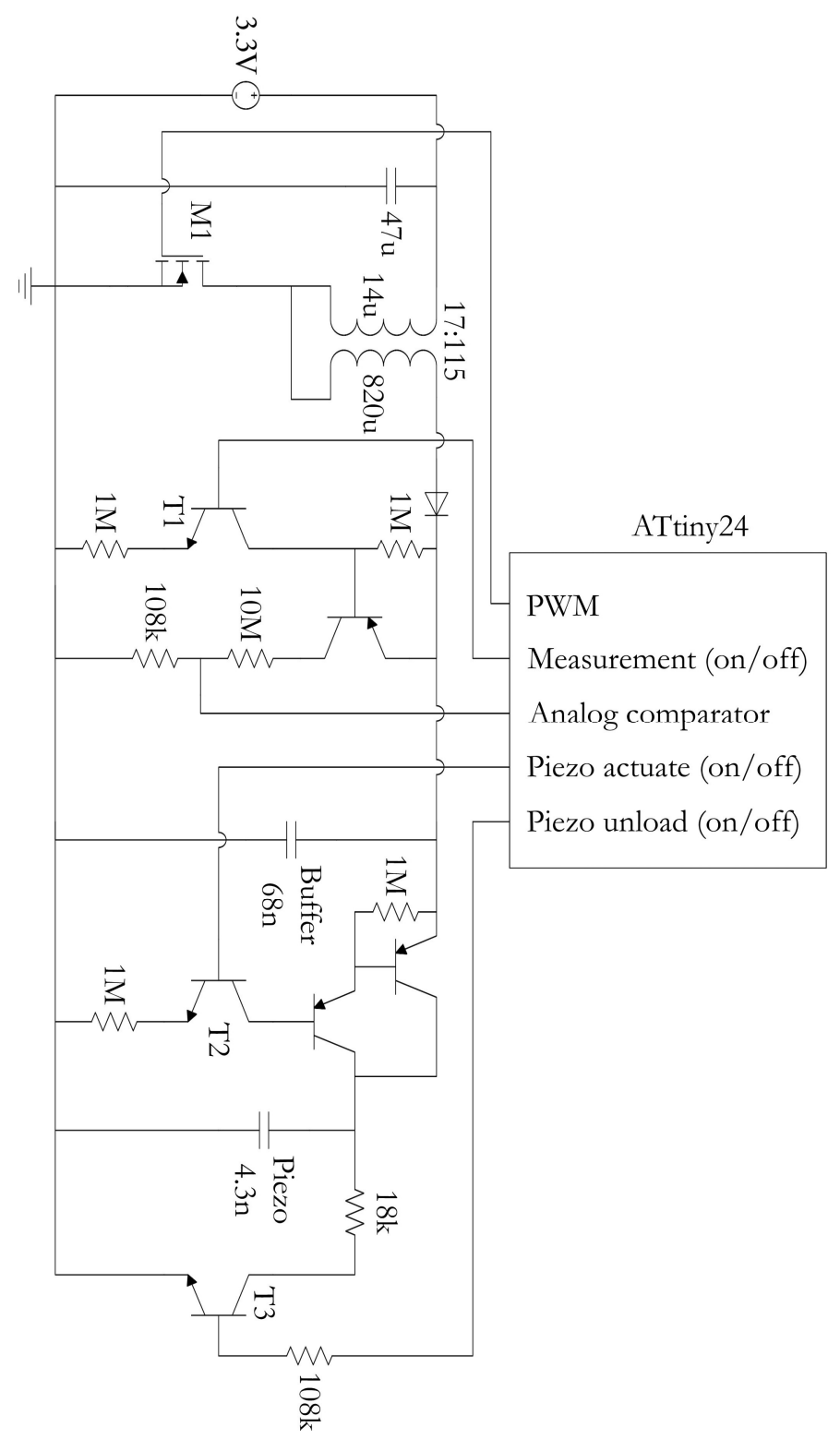

Figure A-I: Electronic circuit of the piezo driver. 


\begin{tabular}{|c|c|c|c|c|}
\hline Component type & number & Brand & Properties & Package \\
\hline Microcontroller & ATtiny24 & Atmel & $\begin{array}{l}\text { PWM, } \\
\text { Analog comparator, } \\
\text { Two-wire communication } \\
8 \text { and 16-bits Timer }\end{array}$ & $\begin{array}{l}\text { QFN, } \\
20 \text { lead, } \\
4 \times 4 \mathrm{~mm}^{2}\end{array}$ \\
\hline Bipolar NPN & BF820 & Philips & $\mathrm{V}_{\text {ce }}$ breakdown: $300 \mathrm{~V}$ & SOT-23 \\
\hline Bipolar PNP & BF821 & Philips & $\mathrm{V}_{\text {ce }}$ breakdown: $-300 \mathrm{~V}$ & SOT-23 \\
\hline $\begin{array}{l}\text { MOSFET } \\
\text { Polarity: N }\end{array}$ & ZXMN3F30FH & Zetex & $\begin{array}{l}\mathrm{V}_{\mathrm{ds}}: 30 \mathrm{~V}, \\
\mathrm{R}_{\mathrm{ds}} \text { on: } 0.047 \Omega\end{array}$ & SOT-23 \\
\hline Diode & ES1GL & $\begin{array}{l}\text { Taiwan } \\
\text { semi. }\end{array}$ & $\begin{array}{l}\text { Fast recovery, } \\
\text { Voltage rating: } 400 \mathrm{~V} \\
\mathrm{~V}_{\mathrm{f}} \max : 1.3 \mathrm{~V}\end{array}$ & SMF \\
\hline Inductor & LQH43M & Murata & $\begin{array}{l}820 \mu \mathrm{H}, 20.5 \Omega, \\
\mathrm{I}_{\mathrm{dc}} \text { max: } 60 \mathrm{~mA} \\
\text { Windings are added to make } \\
\text { a transformer coil }\end{array}$ & 1812 \\
\hline Capacitor & JMK107BJ106MA-T & Yaio Yuden & $\begin{array}{l}\text { Ceramic Multi-Layer, } \\
10 \mu \mathrm{F}, 6.3 \mathrm{~V}\end{array}$ & 0603 \\
\hline Capacitor & 12062C683KAT2A & AVX & $\begin{array}{l}\text { Ceramic Multi-Layer, } \\
68 \mathrm{nF}, 200 \mathrm{~V}\end{array}$ & 1206 \\
\hline Capacitor & C0805C476M9PAC 7800 & Kemet & $\begin{array}{l}\text { Ceramic Multi-Layer, } \\
47 \mu \mathrm{F}, 6.3 \mathrm{~V}\end{array}$ & 0805 \\
\hline Resistor & CRCW06031M00FKEA & $\begin{array}{l}\text { Vishay } \\
\text { Draloric }\end{array}$ & $1 \mathrm{M} \Omega 1 \%, 50 \mathrm{~V}, 0.1 \mathrm{~W}$ & 0603 \\
\hline Resistor & SG73S2BTTD1802F & $\mathrm{KOA}$ & $18 \mathrm{k} \Omega 1 \%, 200 \mathrm{~V}, 0.33 \mathrm{~W}$ & 1206 \\
\hline Resistor & 232272461006 & Phycomp & $10 \mathrm{M} \Omega 1 \%, 200 \mathrm{~V}, 0.25 \mathrm{~W}$ & 1206 \\
\hline Resistor & MC $0.063 \mathrm{~W} 06031 \% 107 \mathrm{~K}$ & Multicomp & $107 \mathrm{k} \Omega 1 \%, 50 \mathrm{~V}, 0.063 \mathrm{~W}$ & 0603 \\
\hline Resistor & CRCW060310R0FKEA & $\begin{array}{l}\text { Vishay } \\
\text { Draloric }\end{array}$ & $10 \Omega 1 \%, 75 \mathrm{~V}, 0.1 \mathrm{~W}$ & 0603 \\
\hline
\end{tabular}

Table A-I: List of electronic components. The most important selection criterion is the package size; the smallest components available are chosen. 


\begin{tabular}{|c|c|}
\hline C-code for the ATtiny24 & Comments \\
\hline $\begin{array}{l}\text { \#include < tiny84.h> } \\
\text { \#include < delay.h }> \\
\text { \#include }<\text { sleep.h }>\end{array}$ & Include libraries \\
\hline $\begin{array}{l}\text { unsigned char wait, state, piezo_on; } \\
\text { unsigned int increment; }\end{array}$ & Declare your global variables \\
\hline $\begin{array}{l}\text { interrupt [ANA_COMP] void ana_comp_isr(void) } \\
\text { wait=0; // end of load_buffer function } \\
\text { state }=1 ; / / \text { voltage }=>\text { required voltage } \\
\text { TCCR0A=0x03; / PWM off } \\
\text { TCCR0B=0x00; } \\
\text { if (!piezo_on }) \\
\{ \\
\text { OCR1AH=0xEA; // 20ms between measurements when loading } \\
\text { OCR1AL=0x60; // Minimize overshoot } \\
\}\end{array}$ & $\begin{array}{l}\text { Analog Comparator interrupt service } \\
\text { routine. } \\
\text { When this int. function is called the voltage } \\
\text { has reached the required value. The PWM } \\
\text { signal is stopped and the time between } \\
\text { measurements is increased to } 60 \mathrm{~ms} \text {. }\end{array}$ \\
\hline $\begin{array}{l}\text { interrupt [TIM1_COMPA] void timer1_compa_isr(void) } \\
\text { PORTA=0x08; // measurement resistances on } \\
\text { delay_us(50); // Required time for } A C \text { int. } \\
\text { PORTA=0x00; // measurement resistances off } \\
\text { increment++; }\end{array}$ & $\begin{array}{l}\text { Timer } 1 \text { output compare } A \text { interrupt service } \\
\text { routine. } \\
\text { Measure voltage }\end{array}$ \\
\hline 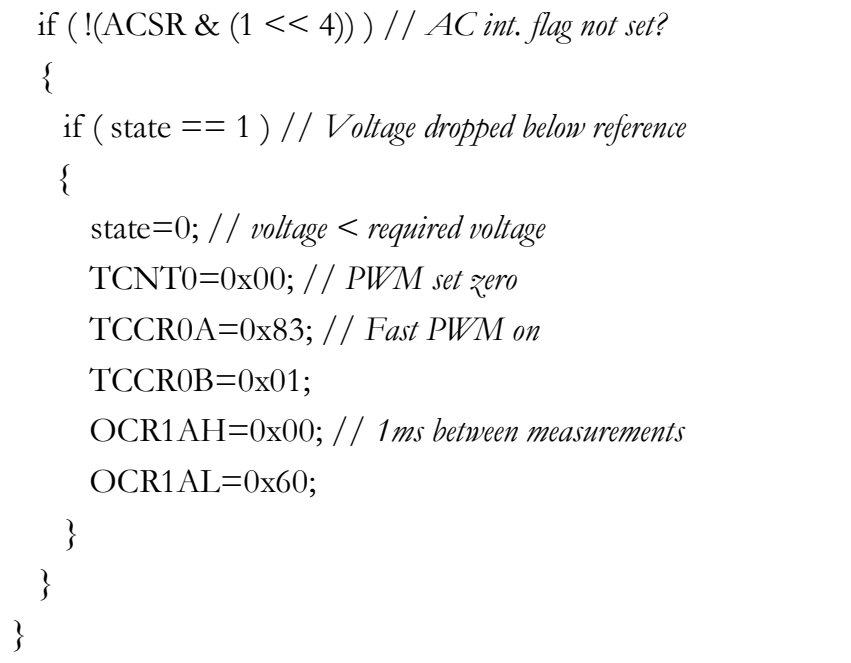 & \\
\hline $\begin{array}{l}\text { void PWM_on(void) } \\
\left\{\begin{array}{l}\text { TCNT0=0x00; // PWM set zero } \\
\text { TCCR0A=0x83; / / Fast PWM on }\end{array}\right.\end{array}$ & $P W M$ signal on \\
\hline
\end{tabular}




\begin{tabular}{|c|c|}
\hline TCCR0B=0x01; & \\
\hline 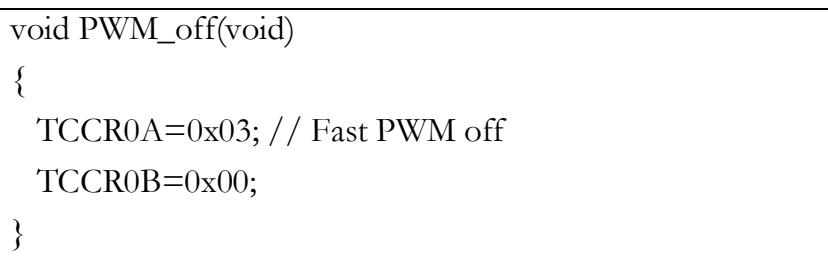 & PWM signal off \\
\hline $\begin{array}{l}\text { void load_buffer(void) } \\
\{ \\
\text { wait=1; } \\
\text { PWM_on } 0 ; \\
\text { PORTA=0x00; // measurement resistances off } \\
\text { TIFR1=0x00; // int. flag reset } \\
\text { TIMSK1=0x02; // timer on, interrupt after } 5 \text { ms } \\
\text { TCNT1H=0x00; // set timer zero } \\
\text { TCNT1L=0x00; } \\
\text { while(wait); } \\
\text { \} }\end{array}$ & Load buffer capacitor for startup \\
\hline $\begin{array}{l}\text { void actuate_piezo(unsigned int open_time) } \\
\text { piezo_on }=1 ; \\
\text { increment }=0 \text {; } \\
\text { ADMUX }=0 x 00 ; / / 180 V \text { adc measurement }\end{array}$ & $\begin{array}{l}\text { Actuate piezo-disc } \\
\text { Use timer for determining open-time (ms). }\end{array}$ \\
\hline $\begin{array}{l}\text { TIFR1=0x00; // int. flag reset } \\
\text { PWM_on0; } \\
\text { delay_ms(20); // Pre-loading to avoid undershoot of pięo voltage }\end{array}$ & \\
\hline $\begin{array}{l}\text { TCNT1H=0x00; / / set counter to zero } \\
\text { TCNT1L=0x00; } \\
\text { TIMSK1 =0x02; } \\
\text { OCR1AH=0x00; // 200us between measurements } \\
\text { OCR1AL=0xC8; } \\
\text { PORTB }=0 \mathrm{x} 01 ; / / \text { piezo-on } \\
\text { while(1); / / increment }<=\text { open_time }\end{array}$ & \\
\hline $\begin{array}{l}\text { PORTB=0x02; / / piezo-off } \\
\text { piezo_on=0; } \\
\text { ADMUX=0x01; / / 190V adc measurement } \\
\text { ? }\end{array}$ & \\
\hline $\begin{array}{l}\text { void unload_buffer(void) } \\
\text { PORTB }=0 \mathrm{x} 00 ; / / \text { idle state } \\
\text { PORTA }=0 \mathrm{x} 08 ; / / \text { unload buffer over measurement resistances }\end{array}$ & Unload buffer capacitor \\
\hline
\end{tabular}




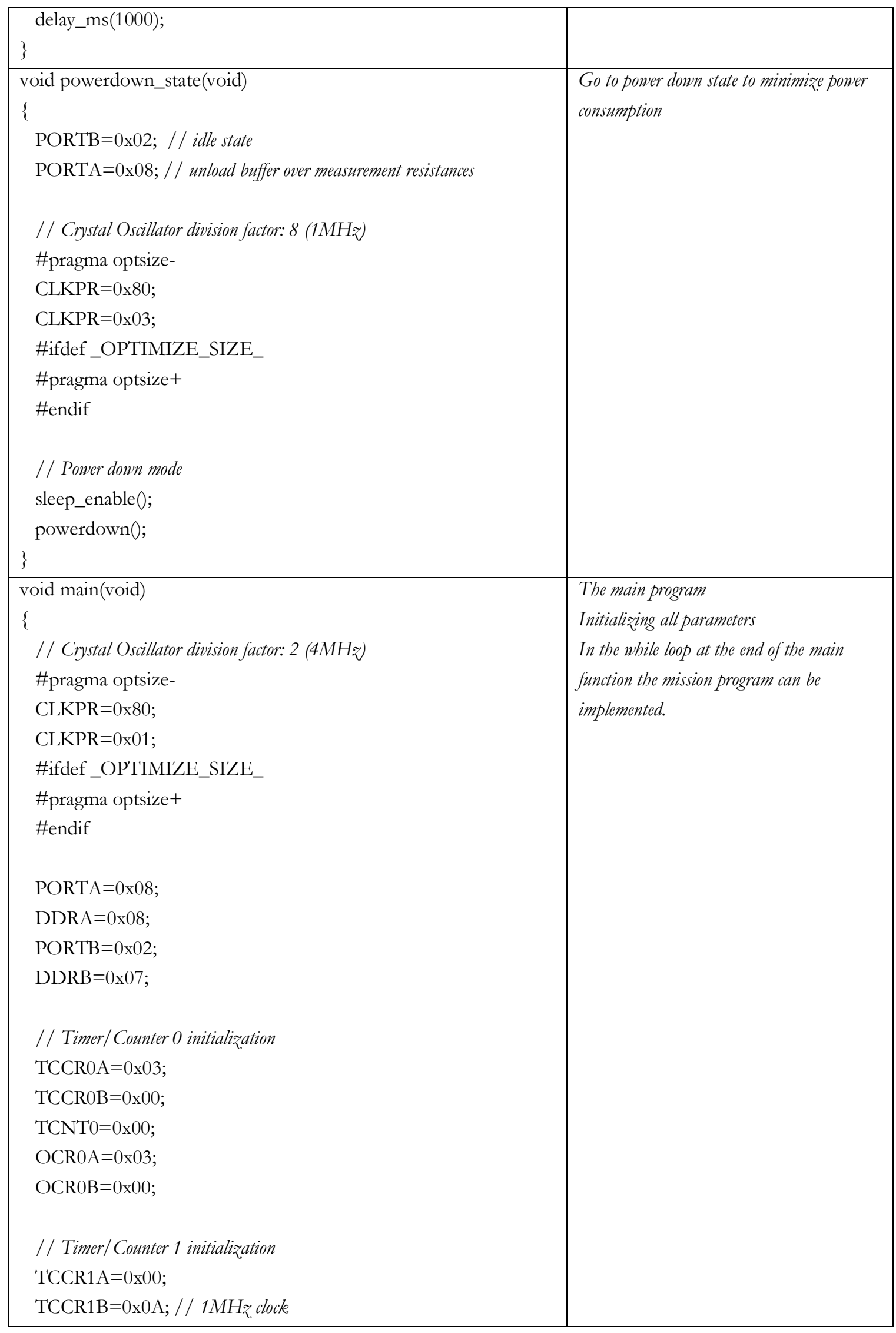




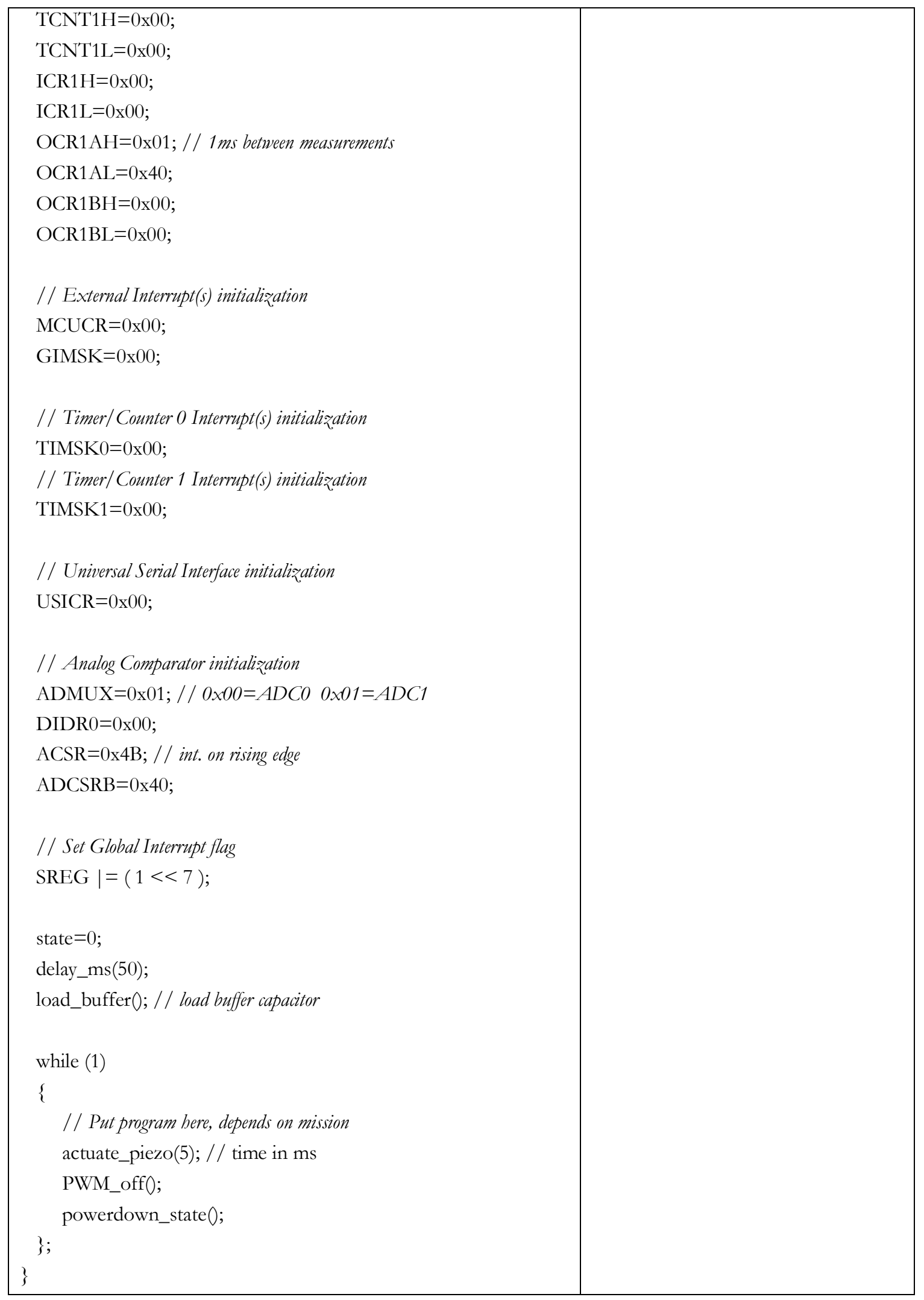

Table A-II: C-code for the ATtiny24 programmed in CodeVision AVR 
Figure A-II shows the measured voltage over the piezo-disc for $100 \mathrm{~ms}$ actuation time. In Figure A-III the rising and falling flanks are shown. Within $400 \mu$ s a stable voltage is reached and within $700 \mu$ s the piezo element is discharged. The ripple on the $180 \mathrm{~V}$ has been measured during actuation. The amplitude of the ripple is $0.5 \mathrm{~V}$.

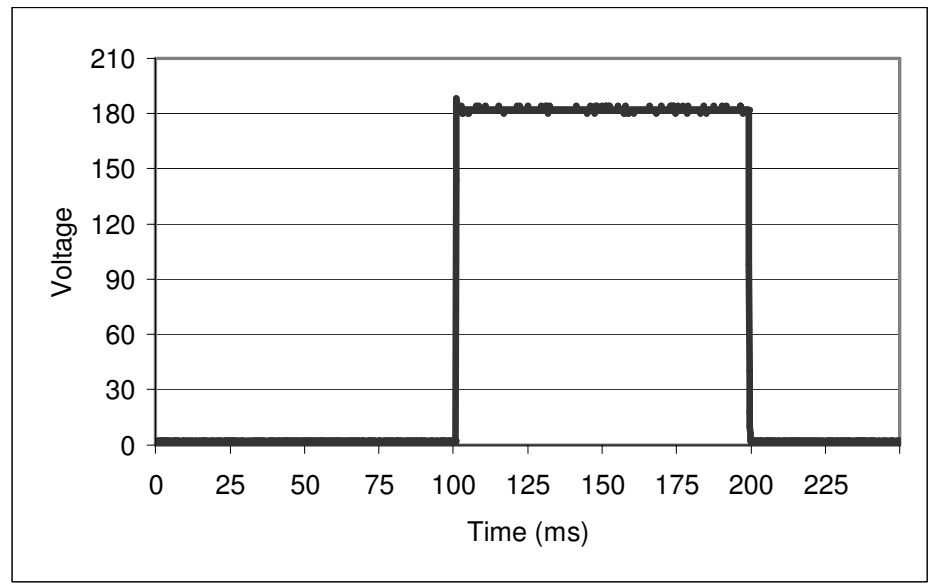

Figure A-II: Voltage over the piezo-disc (100ms open time)

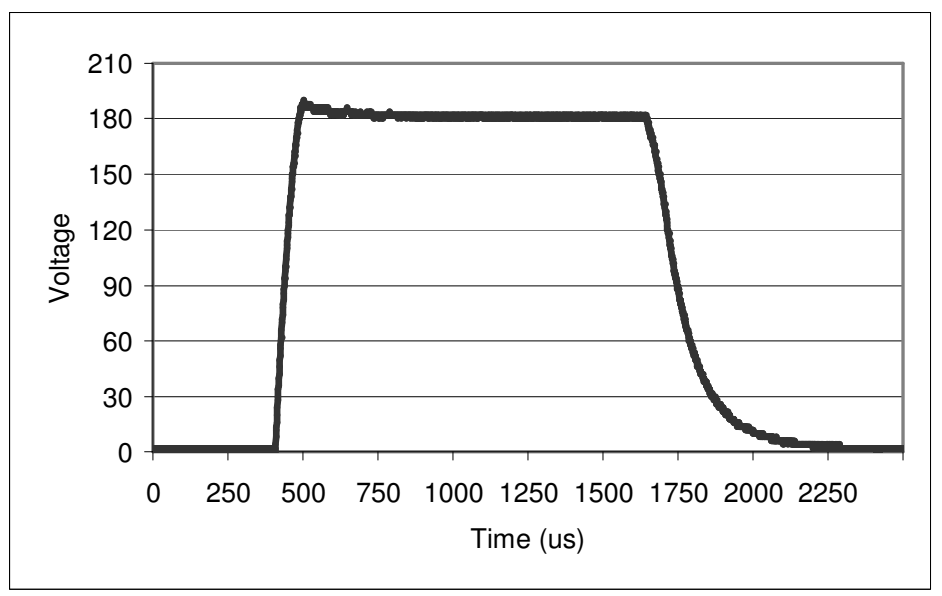

Figure A-III: Voltage over the Piezo-disc (750us open time) 


\section{Appendix B - Glass blowing}

The fabrication of the nozzle by the method of powder blasting and heat treatment revealed a peculiar effect. During the experiments, which led to the glass nozzle shape, some holes were not powder blasted through the wafer. After anodic bonding of the silicon wafer to the glass substrate and a consecutive heat treatment at $790^{\circ} \mathrm{C}$ for $30 \mathrm{~min}$ the nozzle structures where diced to obtain a cross sectional view. The closed cavities change their shape as is visible in Figure B-I. Figure B-I.a shows a cavity before the high temperature step and Figure B-I.b afterwards. During the heat treatment the pressure of entrapped air in the cavity rises significantly while the glass viscosity decreases. At high temperature the glass can reflow and the cavity deforms under the air pressure. Eklund et al used such a glass blowing effect to make spherical microcells [1]. When looking at Figure B-I.b a remarkable effect is observed; a torus shape is formed around the cavity. A shell of the initial cavity is still remaining and in it some small bubbles are visible. It is postulated that the physical properties of the glass shell have been changed by the powder blasting process. Therefore it deforms less easy compared to glass that is not treated by the abrasive $\mathrm{Al}_{2} \mathrm{O}_{3}$ beam. Near the entrance of the powder blasted hole the glass is untreated and can easily deform. At the interface between the silicon and near the entrance of the glass hole the pressurized air deforms the glass resulting in a torus shaped air bubble.

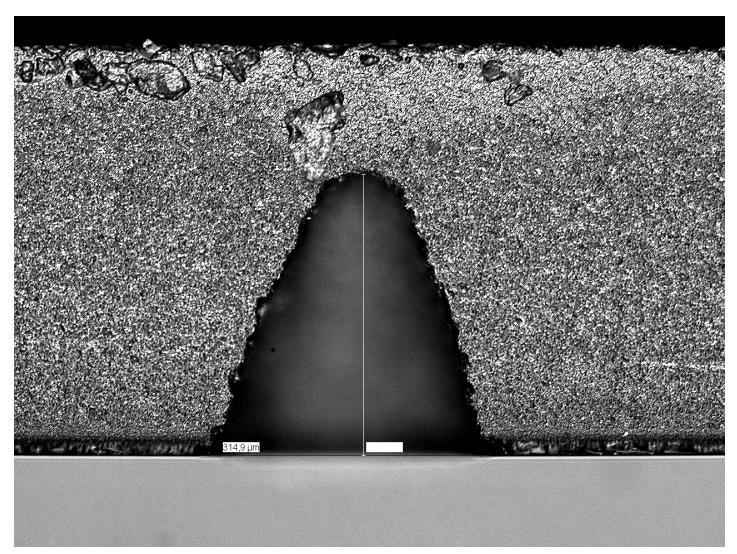

a)

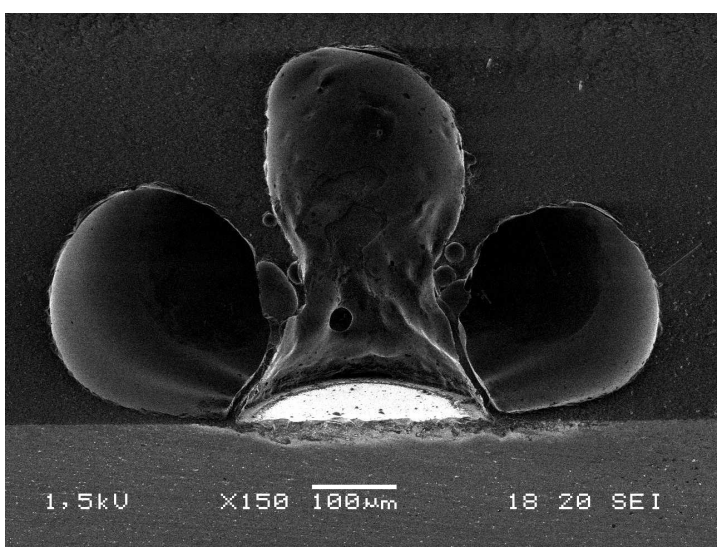

b)

Figure B-I: a) Powder blasted hole before anneal b) SEM image of a closed cavity after heat treatment 
Figure B-II shows cross sectional views of three closed holes after heat treatment. Figure B-II.b corresponds to the SEM picture Figure B-I.b. From the pictures the following is observed. The bigger the initial cavity, the larger the torus lobes and the smaller circular head in the middle. In these pictures one can also see that the surface of the glass wafer is deformed.

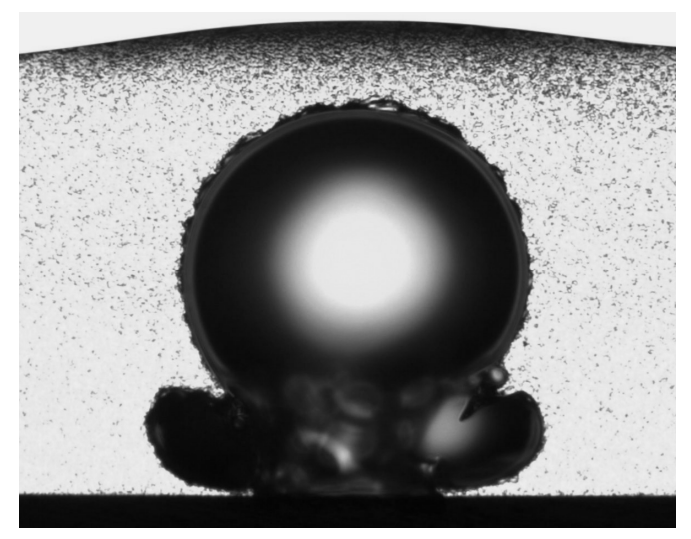

a)

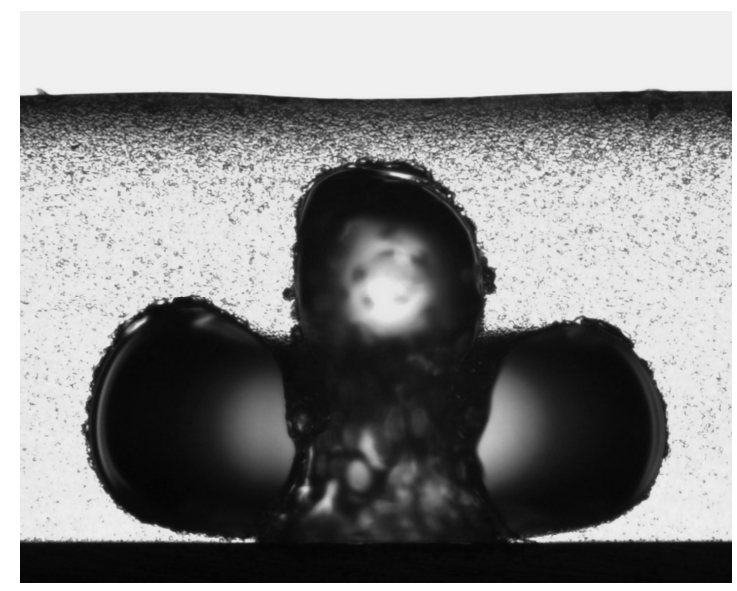

b)

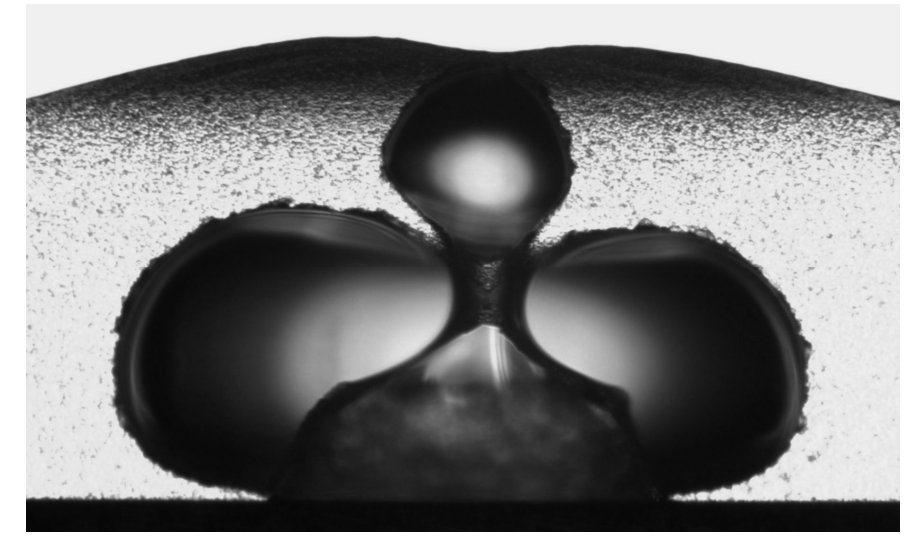

c)

Figure B-II: Closed holes after heat treatment. a) $200 \mu \mathrm{m}$ outlet b) $300 \mu \mathrm{m}$ outlet c) $500 \mu \mathrm{m}$ outlet

[1] Eklund E.J., Shkel A.M., Knappe S., Donley E. and Kitching J., Glass-blown spherical microcells for chip-scale atomic devices, Sensors and Actuators A 143 (2008), pp. 175-180. 


\section{Appendix C - First valve}

Figure C-I shows a schematic of the first generation valve. The glass tube is functioning as a hermetically sealed package, fluidic interconnect as well as a macro support for the fragile MST components [3]. The active valve is normally closed by an embossed membrane which is attached to a piezo-disc. This stack is fixed inside the glass tube by a spring. The spring is made by laser machining of a thin metal sheet and folded into the right shape. It is stacked inside the tube and can exert a force on the valve sealing to repress leakage. The exerted force can be tuned by a screw mechanism. The size of the piezo-disc actuator $(12.7 \mathrm{~mm}$ diameter) determines the diameter of the glass tube which is $13 \mathrm{~mm}$ inner- and $16 \mathrm{~mm}$ outer diameter. When actuated, the piezo-disc pushes against the rigid edge of the membrane and pulls the embossed membrane upwards, which opens the valve, as shown in Figure C-II. Electrical contact with the backside of the piezo-disc is made via the conducting silicon. Electrical contact with the topside of the piezo-disc is made from inside the glass tube. To make the bottom part of the valve rigid enough to avoid deflection under pressure, which causes leakage, it is reinforced by a stack of a glass and silicon disc.

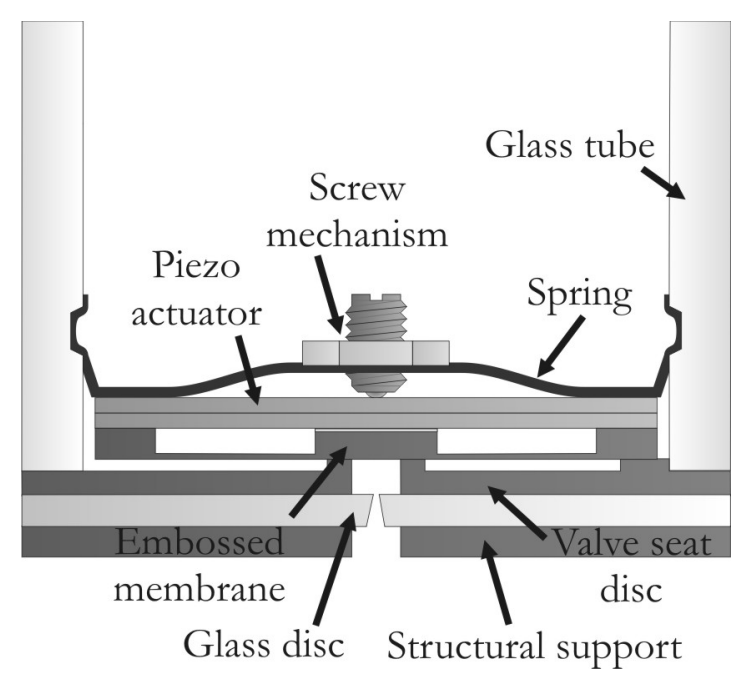

a)

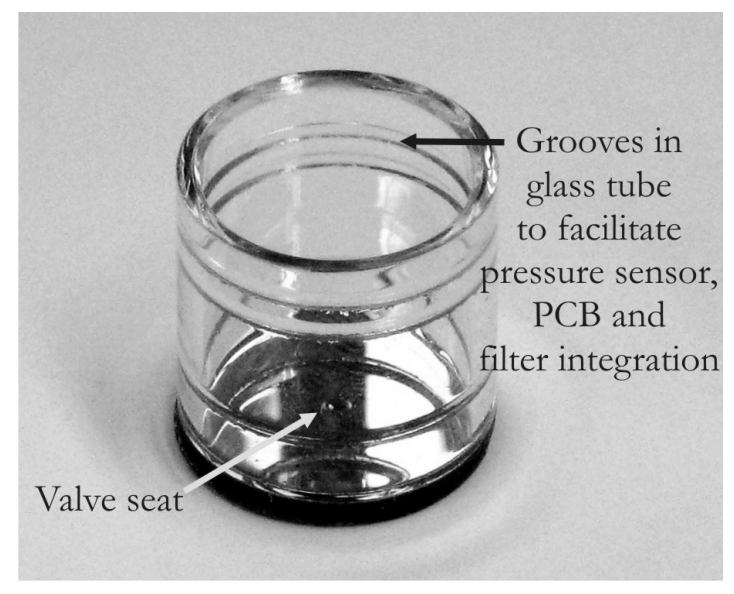

b)

Figure C-I: a) Schematic of valve b) Glass tube on top of the silicon-glass-silicon stack 


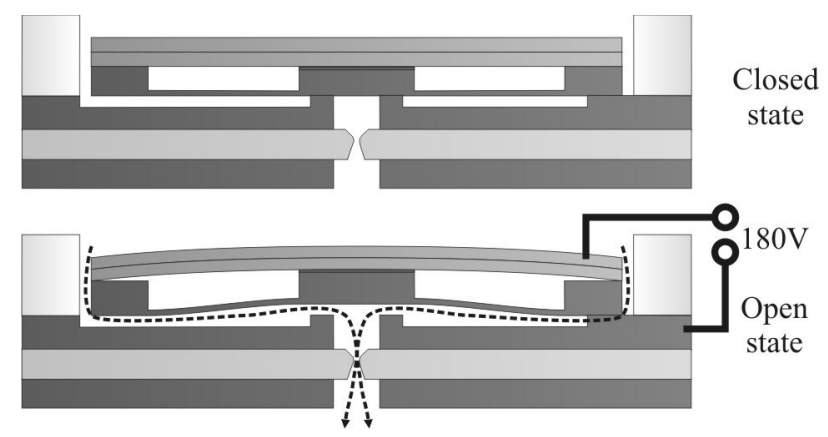

Figure C-II: Schematic of valve actuation

\section{- Fabrication}

The baseline of the thruster and feeding system is a glass machined tube, bonded on the MST part which consists of a silicon-glass-silicon stack as shown in Figure C-I. The silicon wafer containing the valve seat is shaped by two successive reactive ion etching (RIE) steps. The fabrication scheme is shown in Figure C-III. (1) A $500 \mathrm{~nm}$ thick $\mathrm{SiO}_{2}$ layer is grown on a $100 \mathrm{~mm}$ and $525 \mu \mathrm{m}$ thick $<100>$ silicon wafer by wet oxidation. (2) This $\mathrm{SiO}_{2}$ layer is patterned with the shape of the valve seats. (3) An Olin 907-17 mask layer is patterned and used to etch the outlet of the valve and the break trenches. These break trenches are required because we want to get a circular device. With this RIE step the $525 \mu \mathrm{m}$ thick wafer is almost etched through leaving $20 \mu \mathrm{m}$ silicon standing. (4) During a second RIE step a valve seat is shaped using the oxide pattern of step 2 and the valve outlet and break trenches are opened by etching the last $20 \mu \mathrm{m}$ of silicon. A dummy wafer is used to avoid leakage of the Helium backside cooling which is utilized in RIE [BSM VIII]. The dummy wafer is attached to the device wafer by a thin spin coated layer of Fomblin oil, which is normally used as a lubricant in vacuum systems. This additional wafer reduces the heat transfer from the device wafer and causes the devices to break out due to thermal stress when a high power recipe of 2500W is used [11]. By using a $1500 \mathrm{~W}$ recipe the thermal impact is reduced and etching is successful. (5) Finally the $\mathrm{SiO}_{2}$ is removed by $\mathrm{BHF}$. 

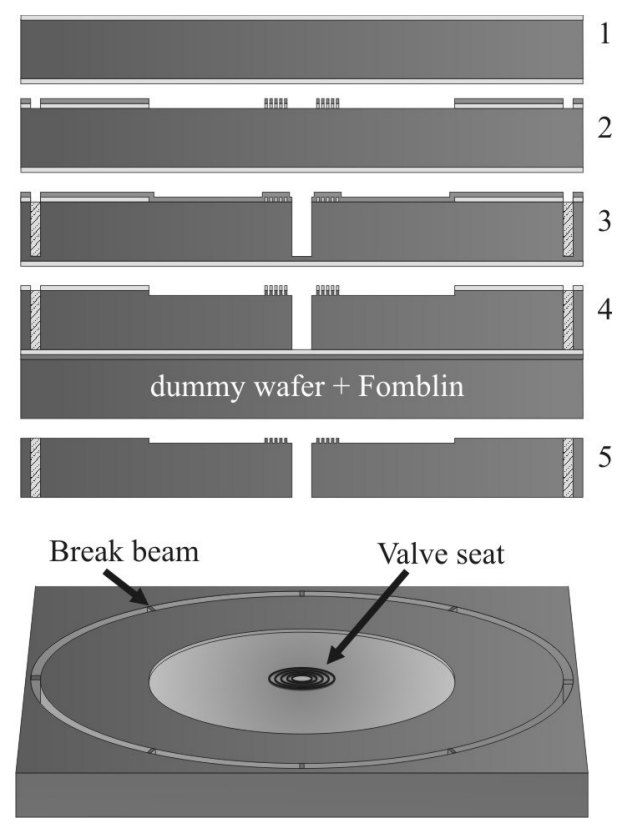

Figure C-III: Valve seat fabrication scheme

In the final thruster and feeding system the glass disc underneath the valve seat (Figure C-I) contains a nozzle but for this prototype the nozzle is not included [5]. Instead of the nozzle, a simple hole is powder blasted in the glass disc functioning as outlet. The silicon support disc (Figure C-I) is manufactured by etching through the wafer by RIE in the same way as described for the silicon disc containing the valve seat. The glass tube is fixed on the MST part by fusion bonding at elevated temperature $[3,12]$. During this high temperature step the two discs that reinforce the valve are also bonded to the valve. Finally, the piezo actuator is attached to the embossed membrane by conductive glue and fixed inside the glass tube by the spring mechanism.

\section{- Actuation measurement}

A specifically designed sample holder is made to connect the gas supply and electrical contacts with the MST valve as shown in Figure C-IV. The bottom part of the holder contains the valve sample. The top part comprises the fluidic interconnect and electrical interface. 

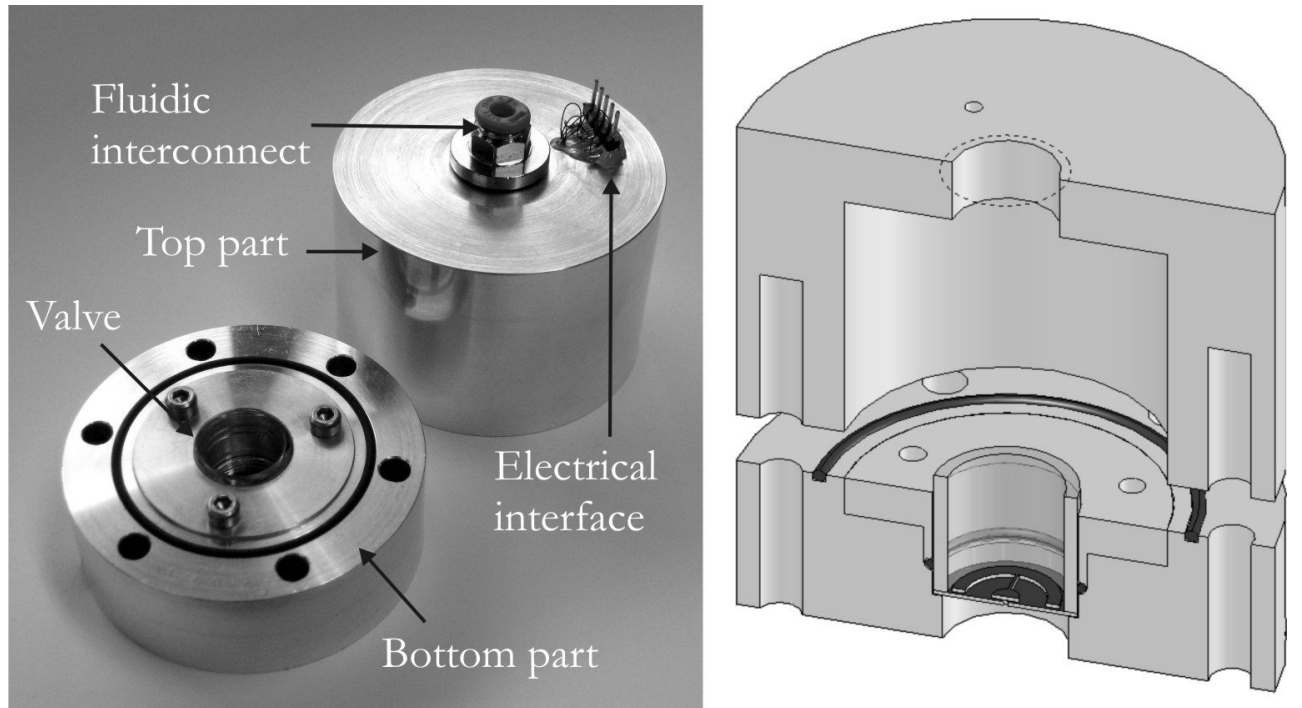

Figure C-IV: Specific sample holder

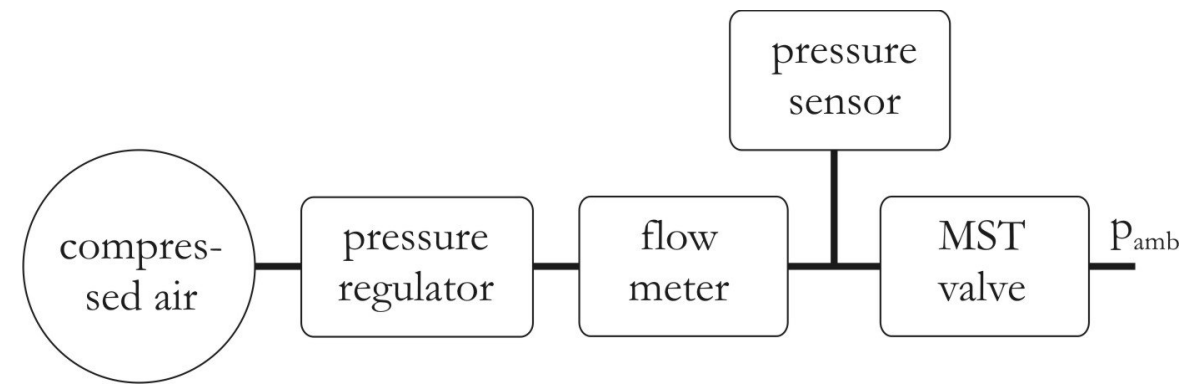

Figure $\boldsymbol{C}$-V: Schematic of the flow measurement setup

The mass flow of air through the valve is measured with the flow measurement setup as shown in Figure $\mathrm{C}-\mathrm{V}$. The outlet of the valve is at ambient pressure, whereas the inlet pressure is controlled by the pressure regulator. The pressure and mass flow are measured at the inlet. The mass flow is measured at 1 bar differential pressure. During the measurement no force is exerted by the spring on the valve sealing. Figure C-VI shows the results of a valve actuated with a block pulse of $150 \mathrm{~V}$ with duration of $20 \mathrm{sec}$ and a $50 \%$ duty cycle. When the valve is opened $(+150 \mathrm{~V})$ the mass flow is out of the range of the flow-meter, above $230 \mathrm{sccm}$. The measured flow when the piezo-disc is relaxed $(0 \mathrm{~V})$ is below $1 \mathrm{sccm}$. Although the measured flow in the closed state is not accurate it is observed that the leakage is much higher than required. 


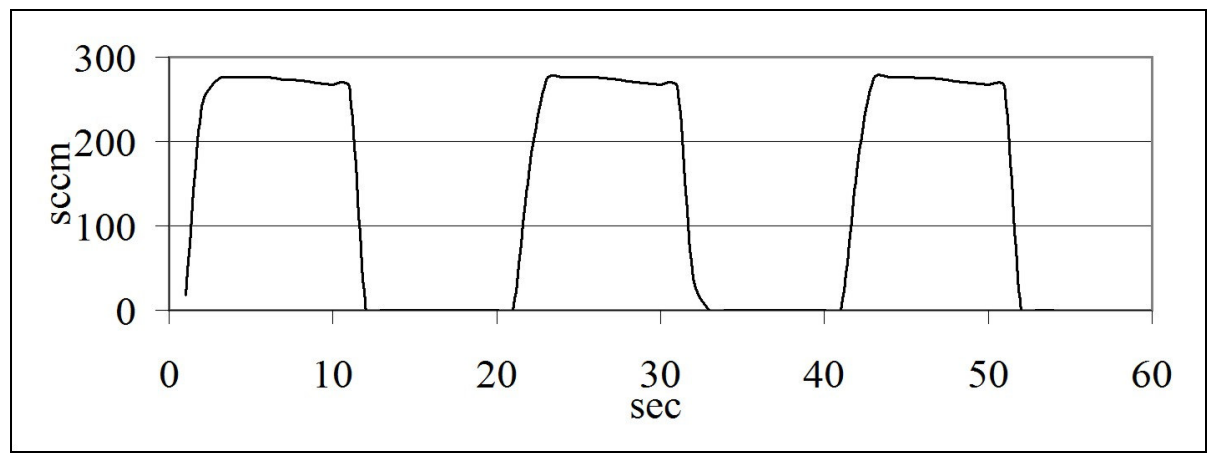

Figure $\boldsymbol{C}$-VI: Flow rate during actuation

- Leakage measurement

To measure the leakage of the valve a sample holder is used wherein the valve is sealed except for the valve outlet as shown in Figure C-VII. For this measurement the top part of the holder contains a pressure sensor. A fluidic interconnect is connected to the bottom part of the sample holder; the outlet of the valve sample.

For this measurement the spring is exerting a considerable force on the valve sealing to see what the minimum leakage is that can be achieved. This force is adjusted by way of the screw mechanism shown in Figure C-I. The valve chamber is pressurized to 1 bar differential pressure through the valve outlet. After filling, the pressure is monitored while the valve is leaking. The result is shown in Figure C-VIII.
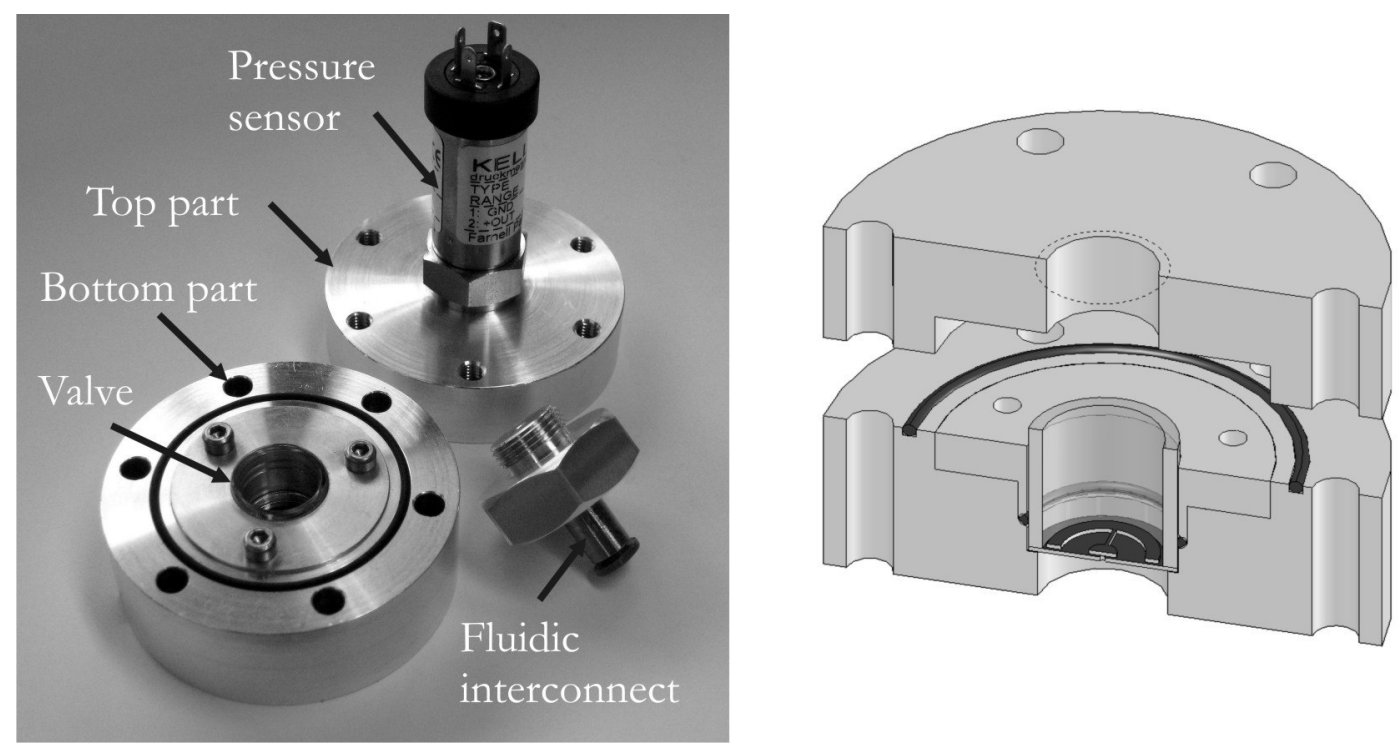

Figure C-VII: Specific sample bolder 


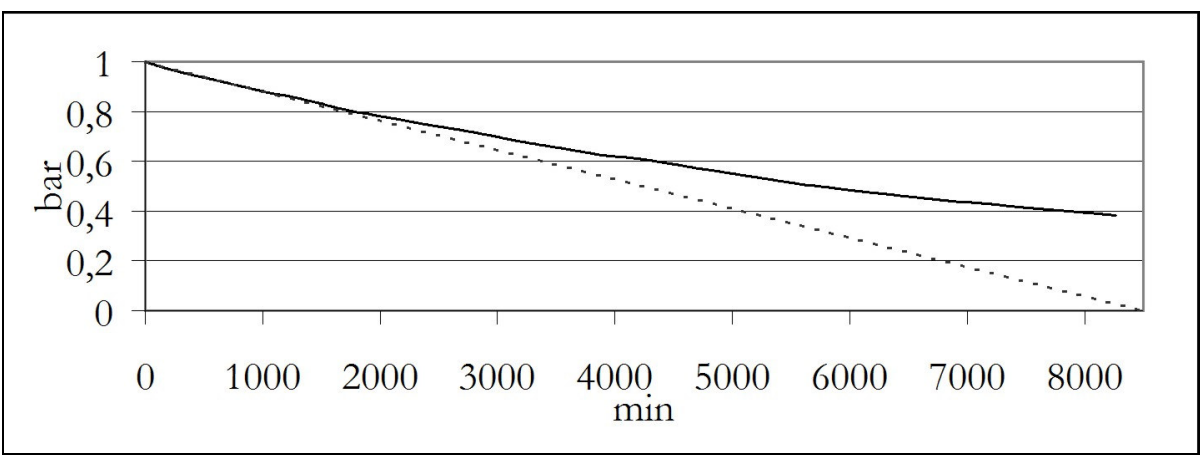

Figure $\boldsymbol{C}$-VIII: V alve leakage

An exponential decay is observed in the pressure and can be described by:

$$
p=p_{0} e^{-t / \tau}
$$

Where $p_{0}$ is the initial pressure, $t$ is the time and $\tau$ is the decay time estimated to be 8500 minutes as indicated by the dashed line in Figure C-VIII. The volume of the valve chamber $(V)$ is approximated by $7 \mathrm{ml}$. The leakage is then given by:

$$
\varphi=\frac{V \cdot p_{0}}{\tau}
$$

The calculated leakage is $8 \cdot 10^{-4} \mathrm{sccm}$ which is 5 times worse than specified.

\section{- Discussion}

As the experiments show the valve is successfully actuated but it shows too high leak-rates in the closed state when no external force is applied. By introducing an external force to close the valve a leak-rate of $8 \cdot 10^{-4} \mathrm{sccm}$ was obtained which is 5 times worse than specified. However, it then became impossible to open the valve since the piezo-actuator generates insufficient force to counter act the external force generated by the spring. The low leakage is quite a strong requirement and not easily satisfied with MST. In conventional valves these low leakages are obtained by using a soft layer which is pressed against a hard valve seat with a very high force [13]. Dirt particles are pressed in the soft layer so they will not cause leakage. A problem with a soft-hard valve is the additional stroke that is required by the actuator. The soft layer is compressed which requires additional stroke. When two hard surfaces are used one needs even more force to crush the dirt particles in order to prevent leakage which further complicates fabrication. Even worse, the valve seat can be damaged due to these high forces as we have seen when applying the external force on the valve seat. 


\section{Appendix D - Stress experiments}

\section{- Introduction}

In chapter 5 thin film membranes were fabricated consisting of $\mathrm{SiO}_{2}$ and $\mathrm{Si}_{3} \mathrm{~N}_{4}$. It is generally believed that the thermal $\mathrm{SiO}_{2}$ has a compressive stress of $0.3 \mathrm{GPa}$ with respect to the silicon substrate, whereas the $\mathrm{Si}_{3} \mathrm{~N}_{4}$ deposited by $\mathrm{LPCVD}$ at $800^{\circ} \mathrm{C}$ would develop a tensile stress of $1.1 \mathrm{GPa}$. For the membranes, the thicknesses of the $\mathrm{SiO}_{2}$ and $\mathrm{Si}_{3} \mathrm{~N}_{4}$ layers were chosen in such a way that both stresses are compensating each other. However, these membranes are exposed to a high temperature anneal at $1100^{\circ} \mathrm{C}$. In our case, this anneal is required for a bonding process. It is found that the stress compensated membranes - although designed to be slightly tensile - became compressive after annealing. This appendix describes initial experiments on this behavior.

For the experiments described, several stress measurements have been done by measuring the surface curvature. The surface curvature is obtained by scanning a needle along the diameter of the wafer surface. The apparatus we use for this is a Veeco Dektak 8. The curvature is measured before and after one side stripping - of both $\mathrm{Si}_{3} \mathrm{~N}_{4}$ and $\mathrm{SiO}_{2}$ - to reveal the film stress, or before and after annealing which is believed to cause a change in stress. The stress in the thin film is then calculated by Stoney's formula [1, 2]:

$$
\sigma_{f}=\frac{4}{3} \frac{Y_{s}}{\left(1-v_{s}\right)} \frac{t_{s}^{2} \delta}{d^{2} t_{f}}=1.04 \cdot 10^{7} \frac{\delta}{t_{f}}
$$

Where $Y_{s}$ is the Young's modulus of the $\mathrm{p}$-type $<100>$ double side polished silicon substrate $(130 \mathrm{GPa}), v_{s}$ the Poisson's ratio of the substrate $(0.28)$ and $t_{s}$ the thickness of the substrate $(525 \mu \mathrm{m})$. The change in deflection is denoted by $\delta, d$ is the scan length $(8 \mathrm{~cm})$ and $t_{f}$ the thickness of the thin film. Over a scan length of $8 \mathrm{~cm}$ the precision of the measurement is within $\pm 1 \mu \mathrm{m}$. This precision is confirmed by repeated scans over a time period of several days. The curvature of the wafers is measured from south to north - where south is the primary flat - and from east to west, where west is the secondary flat. The given deflection is taken at the wafer centre after levelling. The measurements are always taken from the topside of the wafer; having the laser imprinted wafer number. 


\section{- Wafer preparation}

For the experiments we use $<100>525 \pm 5 \mu \mathrm{m}$ silicon substrates. Several layers are deposited and we can distinguish 5 types as shown in Table 2-I. Type1: The thermal oxide (WOX) is grown wet in a steam furnace at $1150^{\circ} \mathrm{C}$. Type2: The TEOS layer is deposited by low pressure chemical vapor deposition (LPCVD) at $700^{\circ} \mathrm{C}$ and consecutively annealed at $1100^{\circ} \mathrm{C}$ for 1 hour. We expect the TEOS to transform into thermal oxide when annealed, due to viscous flow at temperatures above $950^{\circ} \mathrm{C}[3]$. Extra silicon wafers and some of the previous oxidized wafers have been additionally coated with $\mathrm{Si}_{3} \mathrm{~N}_{4}$. The $\mathrm{Si}_{3} \mathrm{~N}_{4}$ is deposited by LPCVD at $800^{\circ} \mathrm{C}$ and thereafter annealed at $1100^{\circ} \mathrm{C}$ for 1 hour. Type $3: \mathrm{Si}_{3} \mathrm{~N}_{4}$ directly on top of the silicon. Type 4: $\mathrm{Si}_{3} \mathrm{~N}_{4}$ on top of WOX and Type 5: $\mathrm{Si}_{3} \mathrm{~N}_{4}$ on top of annealed TEOS. Three wafers of every type are prepared, making a total of 15 test wafers. We introduce a new differentiation by patterning some of the wafers with an $8 \mathrm{~cm}$ ring as shown in Figure D-I.a. For 5 wafers - one of every type - no lithography is done, denoted by the letter N. For another 5 wafers lithography is done only on the backside, i.e. single side lithography (S). And for the last 5 wafers lithography is done on both sides, i.e. double side lithography (D). Figure D-I.b shows the cross section of the different situations.

\begin{tabular}{|l|l|l|l|l|}
\hline Type & Code & Wafer & Layer 1 & Layer 2 \\
\hline 1 & WOX & N1, S1, D1 & $937 \mathrm{~nm}$ thermal oxide $(\mathrm{n}=1.465)$ & \\
\hline 2 & TEOS & N2, S2, D2 & $941 \mathrm{~nm}$ TEOS (annealed) $(\mathrm{n}=1.462)$ & \\
\hline 3 & $\mathrm{Si}_{3} \mathrm{~N}_{4}$ & $\mathrm{~N} 3, \mathrm{~S} 3, \mathrm{D} 3$ & $232 \mathrm{~nm} \mathrm{Si}_{3} \mathrm{~N}_{4}$ (annealed) $(\mathrm{n}=2.021)$ & \\
\hline 4 & $\begin{array}{l}\text { WOX } \\
\mathrm{Si}_{3} \mathrm{~N}_{4}\end{array}$ & $\mathrm{~N} 4, \mathrm{~S} 4, \mathrm{D} 4$ & $937 \mathrm{~nm}$ thermal oxide & $\begin{array}{l}232 \mathrm{~nm} \mathrm{Si}_{3} \mathrm{~N}_{4} \\
\text { (annealed) }\end{array}$ \\
\hline 5 & $\begin{array}{l}\text { TEOS } \\
\mathrm{Si}_{3} \mathrm{~N}_{4}\end{array}$ & $\mathrm{~N} 5, \mathrm{~S} 5, \mathrm{D} 5$ & $941 \mathrm{~nm}$ TEOS (annealed) & $\begin{array}{l}232 \mathrm{~nm} \mathrm{Si} \mathrm{N}_{4} \\
\text { (annealed) }\end{array}$ \\
\hline
\end{tabular}

Table D-I: Test wafer types. Annealed means 1 hour at $1100^{\circ} \mathrm{C}$ in nitrogen atmosphere.

\section{- Results}

The thickness of the thin films has been measured with ellipsometry (Plasmos). The used wavelength of the laser light source is $632.8 \mathrm{~nm}$. Due to the annealing, the TEOS layer gets approximately 3.5\% thinner due to outgassing and rearranging of the atoms which resulted in a layer thickness of $941 \mathrm{~nm}$. The thickness of the 
thermal oxide is measured to be $937 \mathrm{~nm}$ and annealing has no effect on the thickness. As deposited the TEOS has a refractive index of 1.484, while after annealing the value goes to 1.462 , which is close to the refractive index of thermal $\mathrm{SiO}_{2}$ (1.465). Additionally, it is found that BHF etching of annealed TEOS resembles closely the etch rate of thermally grown oxide (about $62 \mathrm{~nm}$ per min). The thickness of $\mathrm{Si}_{3} \mathrm{~N}_{4}-232 \mathrm{~nm}$ - is not affected by additional annealing. The refractive index is measured to be 2.021 and is not changing due to annealing. However, the etch-rate of $\mathrm{Si}_{3} \mathrm{~N}_{4}$ in $50 \% \mathrm{HF}$ seems to halve after 15 hours annealing at $1100^{\circ} \mathrm{C}$ (from 10 to $5.5 \mathrm{~nm}$ per min).

On the next page a table is displayed with the measured deflection data (values are in micrometers). No experiments have been done with wafers N4, D1, D2, D3, S1, S2 and S3 thus they are left out of the table. For the calculation of the change in deflection values are taken from this table. In the calculations, the numbers in between the squared brackets indicate the two columns from which the values are taken. The difference is calculated for two perpendicular measurements and the mean value is calculated. For single thin films - i.e. only $\mathrm{SiO}_{2}$ or $\mathrm{Si}_{3} \mathrm{~N}_{4}-$ this mean value is used to calculate the stress with the help of Stoney's formula. The used layer thicknesses can be found in Table 2-I. The abbreviations bsc and osc mean both sides coated and one side coated, respectively. Where bsc means the wafer had a heat treatment with thin films on both sides and osc means that one side of the wafer was stripped before a heat treatment. Furthermore, for double side patterned wafers the abbreviation $d s l$ ( $l=$ litho) is used and for single side patterned wafers we use ssl. A final remark; be aware that for the initial state, the wafers with $\mathrm{Si}_{3} \mathrm{~N}_{4}$, have already been annealed for $1 \mathrm{hr}$ at $1100^{\circ} \mathrm{C}$.

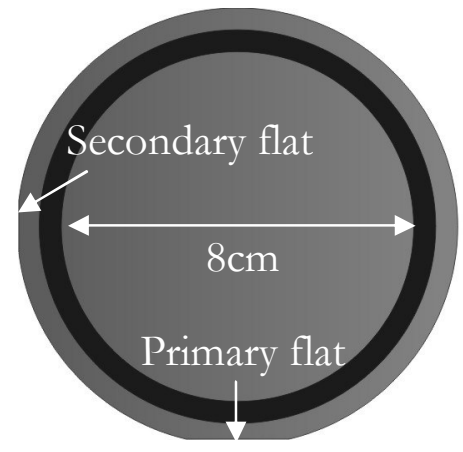

a)

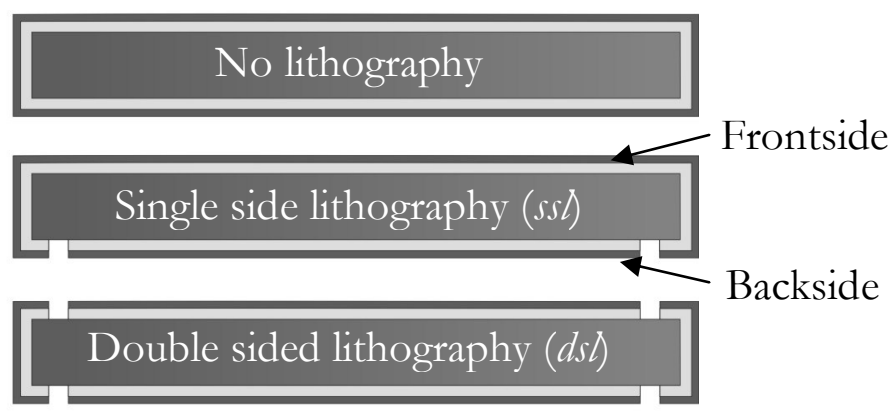

b)

Figure D-I: a) Topside test wafers b) Cross section of the three different situations 


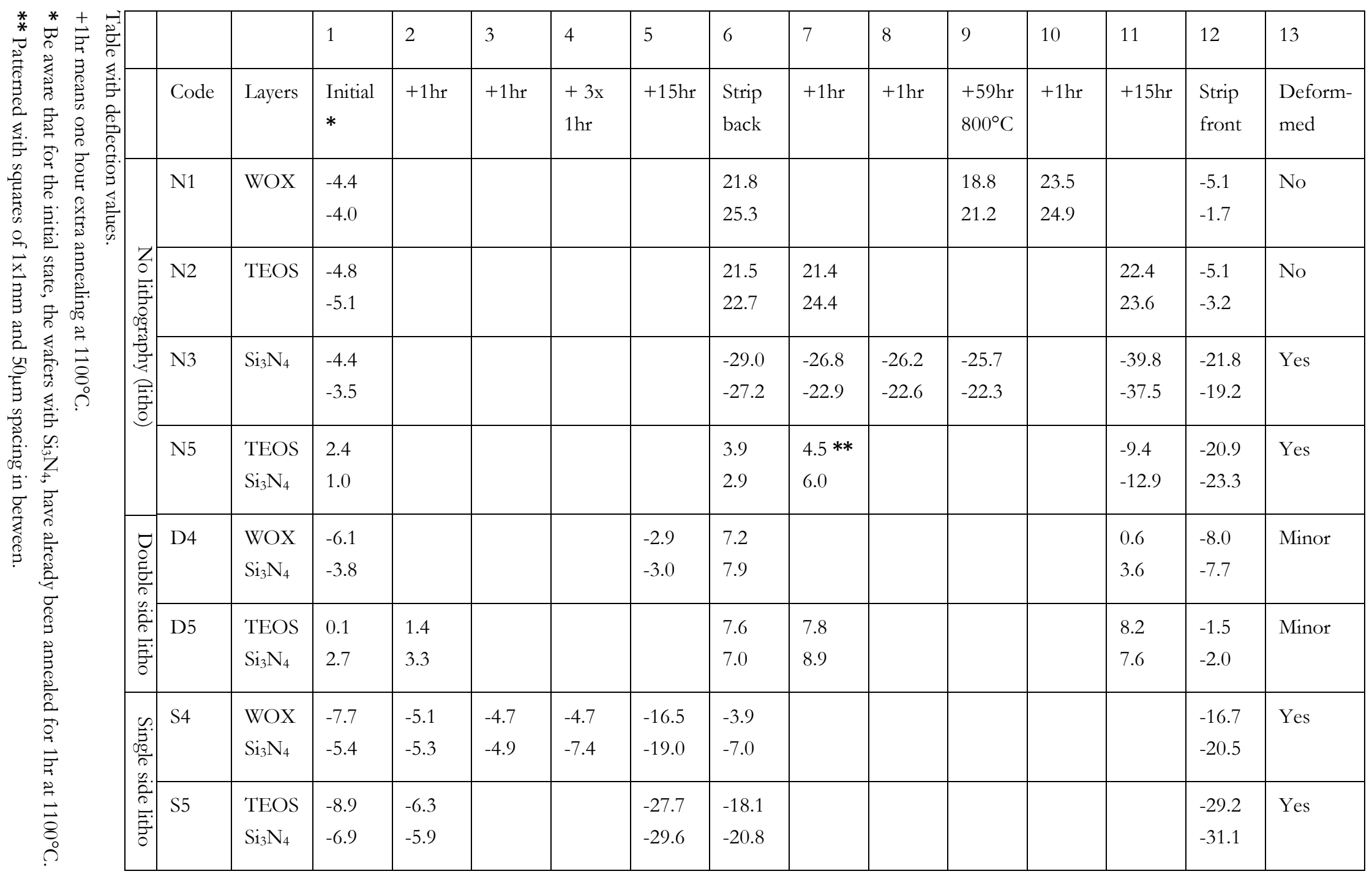




\section{Results from the N-series (no lithography):}

1. N1: WOX strip backside

$\Delta_{a} \delta[6: 1]=(21.8 \pm 1)-(-4.4 \pm 1)=+26.2 \pm 2 \mu \mathrm{m}$

$\Delta_{b} \delta[6: 1]=(25.3 \pm 1)-(-4.0 \pm 1)=+29.3 \pm 2 \mu \mathrm{m}$

$\Delta_{a \nu} \delta[6: 1]=(26.2+29.3) / 2=+27.8 \pm 2 \mu \mathrm{m}$

$\rightarrow \sigma$ WOX,bsc, $1150=-0.31 \pm 0.02 \mathrm{GPa}$

2. N1: +59 hrs annealing backside coated WOX at $800^{\circ} \mathrm{C}$

$\Delta_{a l} \delta[9: 1]=(23.2+25.2) / 2=+24.2 \pm 2 \mu \mathrm{m}$

$\rightarrow \sigma$ WOX,osc, $800=-0.27 \pm 0.02 \mathrm{GPa}$

3. N1: $+1 \mathrm{hr}$ annealing backside coated $\mathrm{WOX}$ at $1100^{\circ} \mathrm{C}$

$\Delta_{a \nu} \delta[10: 1]=(27.9+28.9) / 2=+28.4 \pm 2 \mu \mathrm{m}$

$\rightarrow \sigma$ WOX,osc, $1100=-0.31 \pm 0.02 \mathrm{GPa}$

4. N1: Strip remaining frontside WOX

$\Delta_{a \nu} \delta[12: 10]=(-28.6-26.6) / 2=-27.6 \pm 2 \mu \mathrm{m}$

$\rightarrow \sigma$ WOX,osc, $1100=-0.31 \pm 0.02 \mathrm{GPa}$

5. N1: Compare initial value and all WOX stripped

$\Delta_{a \nu} \delta[12: 1]=(-0.7+2.3) / 2=+0.8 \pm 2 \mu \mathrm{m}$

$\rightarrow$ the silicon wafer is not deformed during several annealing steps

6. N2: TEOS strip backside

$\Delta_{a l} \delta[6: 1]=(26.3+27.8) / 2=+27.1 \pm 2 \mu \mathrm{m}$

$\rightarrow \sigma$ TEOS,bsc, $1100=-0.30 \pm 0.02 \mathrm{GPa}$

7. N2: $+1+15$ hrs annealing backside coated TEOS at $1100^{\circ} \mathrm{C}$

$\Delta_{a \nu} \delta[11: 1]=(27.2+28.7) / 2=+28.0 \pm 2 \mu \mathrm{m}$

$\rightarrow \sigma$ TEOS,osc, $1100=-0.31 \pm 0.02 \mathrm{GPa}$

8. N2: Strip remaining frontside TEOS

$\Delta_{a l} \delta[12: 11]=(-27.5-26.8) / 2=-27.2 \pm 2 \mu \mathrm{m}$

$\rightarrow \sigma$ TEOS,osc, $1100=-0.30 \pm 0.02 \mathrm{GPa}$

9. N2: Compare initial value and all TEOS stripped

$\Delta_{a v} \delta[12: 1]=(-0.3+1.9) / 2=+0.8 \pm 2 \mu \mathrm{m}$

$\rightarrow$ the silicon wafer is not deformed during several annealing steps

10. N3: $\underline{\mathbf{S i}}_{3} \underline{\mathbf{N}}_{4}$ strip backside

$\Delta_{a \nu} \delta[6: 1]=(-24.6-23.7) / 2=-24.2 \pm 2 \mu \mathrm{m}$

$\rightarrow \sigma{ }_{s i 3 N 4, b s c, 1100}=+1.08 \pm 0.09 \mathrm{GPa}$ 
11. N3: $+3+15$ hrs annealing backside coated $\mathrm{Si}_{3} \mathrm{~N}_{4}$ at $1100^{\circ} \mathrm{C}$

and strip remaining frontside $\mathrm{Si}_{3} \mathrm{~N}_{4}$

$\Delta_{a \imath} \delta[12: 11]=(18.0+18.3) / 2=+18.2 \pm 2 \mu \mathrm{m}$

$\rightarrow \sigma$ si3N4,oss, $1100=+0.81 \pm 0.09 \mathrm{GPa}$

12. N3: Compare initial value and all $\mathrm{Si}_{3} \mathrm{~N}_{4}$ stripped

$\Delta_{a \nu} \delta[12: 1]=(-17.4-15.7) / 2=-16.6 \pm 2 \mu \mathrm{m}$

$\rightarrow$ the silicon wafer is heavily deformed during several annealing steps

13. N5: $\underline{\mathbf{S i}}_{3} \underline{\mathbf{N}}_{4}$ on top of TEOS strip both layers backside

$\Delta_{a \nu} \delta[6: 1]=(1.5+1.9) / 2=1.7 \pm 2 \mu \mathrm{m}$

The stress in the $\mathrm{Si}_{3} \mathrm{~N}_{4}$ is calculated by using result 6 and 10

$\rightarrow \sigma$ Si3N4 on TEOS,bsc, $1100=1.08^{*}(27.1-1.7) / 24.2=+1.14 \pm 0.09 \mathrm{GPa}$

14. N5: **Front patterned with squares of $1 \mathrm{x} 1 \mathrm{~mm}$ with $50 \mu \mathrm{m}$ spacing in between.

$+1+15$ hrs annealing at $1100^{\circ} \mathrm{C}$ of $\mathrm{Si}_{3} \mathrm{~N}_{4}$ on top of TEOS

and strip remaining frontside $\mathrm{Si}_{3} \mathrm{~N}_{4}$ and TEOS

$\Delta_{a l} \delta[12: 11]=(-11.5-10.4) / 2=-11.0 \pm 2 \mu \mathrm{m}$

$\rightarrow \sigma$ Si3N4 on TEOS,oss, $1100=1.08 *(27.1-11.0) / 24.2=+0.72 \pm 0.09 \mathrm{GPa}$

15. N5: Compare initial value and all $\mathrm{Si}_{3} \mathrm{~N}_{4}$ and TEOS stripped

$\Delta_{a l} \delta[12: 1]=(-23.3-24.3) / 2=-23.8 \pm 2 \mu \mathrm{m}$

$\rightarrow$ the silicon wafer is heavily deformed during several annealing steps

\section{Results from the D-series (double side lithography):}

16. D4: $\underline{\mathbf{S i}}_{3} \underline{\mathbf{N}}_{4}$ on top of WOX $+15 \mathrm{hrs}$ annealing at $1100^{\circ} \mathrm{C}$ and backside strip

$\Delta_{a \iota} \delta[6: 5]=(10.1+10.9) / 2=+10.5 \pm 2 \mu \mathrm{m}$

The stress in the $\mathrm{Si}_{3} \mathrm{~N}_{4}$ is calculated by using result 1 and 10

$\rightarrow \sigma$ Si3N4 on WOX,bsc, $1100=1.08 *(27.8-10.5) / 24.2=0.77 \pm 0.09 \mathrm{GPa}$

17. D4: +15 hrs extra annealing one side coated $\mathrm{Si}_{3} \mathrm{~N}_{4}$ on top of WOX at $1100^{\circ} \mathrm{C}$ and strip remaining frontside $\mathrm{Si}_{3} \mathrm{~N}_{4}$ and WOX

$\Delta_{a \nu} \delta[12: 11]=(-8.6-11.3) / 2=-10.0 \pm 2 \mu \mathrm{m}$

$\rightarrow \sigma$ si3N4 on WOX,bsc, $1100=1.08 *(27.8-10.0) / 24.2=0.80 \pm 0.09 \mathrm{GPa}$

18. D4: Compare initial value and all $\mathrm{Si}_{3} \mathrm{~N}_{4}$ and WOX stripped

$\Delta_{a l} \delta[12: 1]=(-1.9-3.9) / 2=-2.9 \pm 2 \mu \mathrm{m}$

$\rightarrow$ the silicon wafer is slightly deformed during several annealing steps

19. D5: $\underline{\mathbf{S i}}_{3} \underline{\mathbf{N}}_{4} \underline{\text { on top of } \mathbf{T E O S}}+1 \mathrm{hr}$ annealing at $1100^{\circ} \mathrm{C}$ and backside layers strip $\Delta_{a v} \delta[6: 2]=(7.5+4.3) / 2=+5.9 \pm 2 \mu \mathrm{m}$ 
The stress in the $\mathrm{Si}_{3} \mathrm{~N}_{4}$ is calculated by using result 6 and 10 $\rightarrow \sigma$ Si3N4 on $W O X, b s, 1100=1.08 *(27.1-5.9) / 24.2=0.95 \pm 0.09 \mathrm{GPa}$

20. D5: $+1+15 \mathrm{hrs}$ annealing one side coated $\mathrm{Si}_{3} \mathrm{~N}_{4}$ on top of TEOS at $1100^{\circ} \mathrm{C}$ and strip remaining frontside $\mathrm{Si}_{3} \mathrm{~N}_{4}$ and TEOS

$\Delta_{a \jmath} \delta[12: 11]=(-9.7-9.6) / 2=-9.7 \pm 2 \mu \mathrm{m}$

$\rightarrow \sigma$ Si3N4 on WOX,bsc, $1100=1.08 *(27.1-9.7) / 24.2=0.78 \pm 0.09 \mathrm{GPa}$

21. D5: Compare initial value and all $\mathrm{Si}_{3} \mathrm{~N}_{4}$ and TEOS stripped

$\Delta_{a \diamond} \delta[12: 1]=(-1.6-4.7) / 2=-3.2 \pm 2 \mu \mathrm{m}$

$\rightarrow$ the silicon wafer is slightly deformed during several annealing steps

\section{Results from the S-series (single side lithography):}

22. S4: $\underline{\mathbf{S}}_{3} \underline{\mathbf{N}}_{4}$ on top of WOX $+5^{*} 1+15 \mathrm{hrs}$ annealing at $1100^{\circ} \mathrm{C}$ and backside strip

$\Delta_{a \nu} \delta[6: 5]=(12.6+12.0) / 2=+12.3 \pm 2 \mu \mathrm{m}$

The stress in the $\mathrm{Si}_{3} \mathrm{~N}_{4}$ is calculated by using result 1 and 10

$\rightarrow \sigma$ Si3N4 on WOX,bsc, $1100=1.08 *(27.8-12.3) / 24.2=0.69 \pm 0.09 \mathrm{GPa}$

23. S4: Strip remaining frontside $\mathrm{Si}_{3} \mathrm{~N}_{4}$ and WOX

$\Delta_{a \prime \prime} \delta[12: 6]=(12.8+13.5) / 2=+13.2 \pm 2 \mu \mathrm{m}$

$\rightarrow \sigma$ Si3NA on $W O X$, bsc, $1100=1.08 *(27.8-13.2) / 24.2=0.65 \pm 0.09 \mathrm{GPa}$

24. S4: Compare initial value and all $\mathrm{Si}_{3} \mathrm{~N}_{4}$ and WOX stripped

$\Delta_{a l} \delta[12: 1]=(-9.0-15.1) / 2=-12.1 \pm 2 \mu \mathrm{m}$

$\rightarrow$ the silicon wafer is heavily deformed during several annealing steps

25. S5: $\underline{\mathrm{Si}}_{3} \underline{\mathbf{N}}_{4}$ on top of TEOS $+1+15 \mathrm{hrs}$ annealing at $1100^{\circ} \mathrm{C}$ and backside strip $\Delta_{a l} \delta[6: 5]=(9.6+8.8) / 2=+9.2 \pm 2 \mu \mathrm{m}$

The stress in the $\mathrm{Si}_{3} \mathrm{~N}_{4}$ is calculated by using result 1 and 10

$\rightarrow \sigma$ Si3N4 on TEOS,bss, $1100=1.08 *(27.1-9.2) / 24.2=0.80 \pm 0.09 \mathrm{GPa}$

26. S5: Strip remaining frontside $\mathrm{Si}_{3} \mathrm{~N}_{4}$ and TEOS

$\Delta_{a \curlywedge} \delta[12: 6]=(11.1+10.3) / 2=+10.7 \pm 2 \mu \mathrm{m}$

$\rightarrow \sigma$ Si3N4 on WOX,bss, $1100=1.08 *(27.1-10.7) / 24.2=0.73 \pm 0.09 \mathrm{GPa}$

27. S5: Compare initial value and all $\mathrm{Si}_{3} \mathrm{~N}_{4}$ and WOX stripped

$\Delta_{a i \delta}[12: 1]=(-20.3-24.2) / 2=-22.3 \pm 2 \mu \mathrm{m}$

$\rightarrow$ the silicon wafer is heavily deformed during several annealing steps 


\begin{tabular}{|c|c|c|c|c|c|c|c|c|c|c|c|c|c|c|c|c|}
\hline 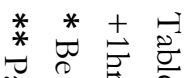 & & & & 1 & 2 & 3 & 4 & 5 & 6 & 7 & 8 & 9 & 10 & 11 & 12 & 13 \\
\hline 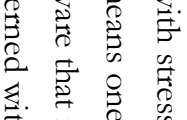 & & Code & Layers & $\begin{array}{l}\text { Initial } \\
*\end{array}$ & $+1 \mathrm{hr}$ & $+1 \mathrm{hr}$ & $\begin{array}{l}+3 \mathrm{x} \\
1 \mathrm{hr}\end{array}$ & $+15 \mathrm{hr}$ & $\begin{array}{l}\text { Strip } \\
\text { back }\end{array}$ & $+1 \mathrm{hr}$ & $+1 \mathrm{hr}$ & $\begin{array}{l}+59 \mathrm{hr} \\
800^{\circ} \mathrm{C}\end{array}$ & $+1 \mathrm{hr}$ & $+15 \mathrm{hr}$ & $\begin{array}{l}\text { Strip } \\
\text { front }\end{array}$ & $\begin{array}{l}\text { Deform- } \\
\text { med }\end{array}$ \\
\hline 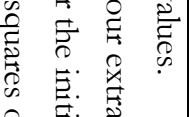 & & $\mathrm{N} 1$ & WOX & -0.31 & & & & & -0.31 & & & -0.27 & -0.31 & & -0.31 & $\begin{array}{l}\text { No } \\
0.8\end{array}$ \\
\hline 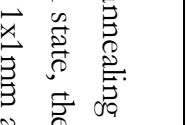 & 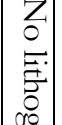 & $\mathrm{N} 2$ & TEOS & -0.30 & & & & & -0.30 & -0.31 & & & & -0.31 & -0.30 & $\begin{array}{l}\text { No } \\
0.8\end{array}$ \\
\hline 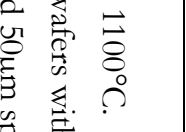 & 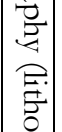 & N3 & $\mathrm{Si}_{3} \mathrm{~N}_{4}$ & 1.08 & & & & & 1.08 & & & & & 0.81 & 0.81 & $\begin{array}{l}\text { Yes } \\
-16.6\end{array}$ \\
\hline 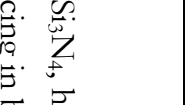 & & N5 & $\begin{array}{l}\text { TEOS } \\
\mathrm{Si}_{3} \mathrm{~N}_{4}\end{array}$ & 1.14 & & & & & 1.14 & $* *$ & & & & 0.72 & 0.72 & $\begin{array}{l}\text { Yes } \\
-23.8\end{array}$ \\
\hline 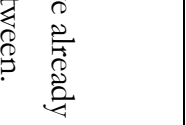 & $\left|\begin{array}{l}E \\
0 \\
0 \\
\frac{D}{0} \\
\end{array}\right|$ & D4 & $\begin{array}{l}\text { WOX } \\
\mathrm{Si}_{3} \mathrm{~N}_{4}\end{array}$ & "1.1" & & & & 0.77 & 0.77 & & & & & 0.80 & 0.80 & $\begin{array}{l}\text { Minor } \\
-2.9\end{array}$ \\
\hline $\begin{array}{l}\vec{y} \\
\tilde{\tilde{E}} \\
\overrightarrow{0}\end{array}$ & 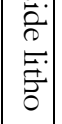 & D5 & $\begin{array}{l}\text { TEOS } \\
\mathrm{Si}_{3} \mathrm{~N}_{4}\end{array}$ & “1.1" & 0.95 & & & & 0.95 & & & & & 0.78 & 0.78 & $\begin{array}{l}\text { Minor } \\
-3.4\end{array}$ \\
\hline 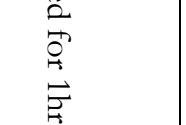 & 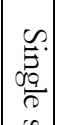 & S4 & $\begin{array}{l}\text { WOX } \\
\mathrm{Si}_{3} \mathrm{~N}_{4}\end{array}$ & "1.1" & & & & 0.69 & 0.69 & & & & & & 0.65 & $\begin{array}{l}\text { Yes } \\
-12.1\end{array}$ \\
\hline $\begin{array}{l}\overrightarrow{\overrightarrow{8}} \\
0 \\
0\end{array}$ & 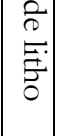 & S5 & $\begin{array}{l}\text { TEOS } \\
\mathrm{Si}_{3} \mathrm{~N}_{4}\end{array}$ & "1.1" & & & & 0.8 & 0.8 & & & & & & 0.73 & $\begin{array}{l}\text { Yes } \\
-22.3\end{array}$ \\
\hline
\end{tabular}


- Conclusions

\section{The following conclusions are made from the $\mathrm{N}$-series.}

1. ( $\boldsymbol{W O X}$ result 1) Wet thermal oxide (WOX) grown at $1150^{\circ} \mathrm{C}$ has a stress of $0.31 \pm 0.02 \mathrm{GPa}[4]$.

2. ( $\boldsymbol{W O X}$ result 2) When WOX is annealed afterwards at $800^{\circ} \mathrm{C}$ the stress decreases to $-0.27 \pm 0.02 \mathrm{GPa}$. This is explained by the notion that the $\mathrm{SiO}_{2}$ film is slowly rearranging its structure and releasing stress to converge to a stress free situation at $800^{\circ} \mathrm{C}$. Since the stress is only caused by differences in thermal expansion, cooling down from $800^{\circ} \mathrm{C}$ to room temperature results in a lower stress buildup than when it is cooled down from $1100^{\circ} \mathrm{C}$ [3].

3. (WOX result 3) When the WOX - after the $800^{\circ} \mathrm{C}$ anneal - is again annealed at $1100^{\circ} \mathrm{C}$ the stress returns to its original value of $-0.31 \pm 0.02 \mathrm{GPa}$.

4. (TEOS result 6 and 7) TEOS annealed for $>1$ hour at $1100^{\circ} \mathrm{C}$ develops the same stress as WOX and becomes indistinguishable. Therefore, we consider annealed TEOS and WOX as similar for the following conclusions.

5. (WOX and TEOS result 1, 4, 6 and 8) Annealing of a one side $\mathrm{SiO}_{2}$ coated wafer has the same stress value as for both side coated wafers.

6. (WOX and TEOS result 5 and 9) Annealing of a one side $\mathrm{SiO}_{2}$ coated wafer does not deform silicon. This is explained by the fact that the oxide is stress free at $1100^{\circ} \mathrm{C}$ and, so, the wafer is not under strain.

7. (Sij $\boldsymbol{N}_{4}$ result 10 and 13 ) Stoichiometric LPCVD $\mathrm{Si}_{3} \mathrm{~N}_{4}$ has a stress value of $1.1 \pm 0.09 \mathrm{GPa}$ both on silicon and TEOS. Note that this is after an hour annealing as this was the initial condition.

8. ( $\boldsymbol{S i}_{3} \boldsymbol{N}_{4}$ result 11 ) Annealing at $1100^{\circ} \mathrm{C}$ seems to release part of the $\mathrm{Si}_{3} \mathrm{~N}_{4}$ stress. After $3+15$ hours annealing the $\mathrm{Si}_{3} \mathrm{~N}_{4}$ stress lowers to $0.81 \pm 0.09 \mathrm{GPa}$.

9. ( $\boldsymbol{S i}_{3} \mathbf{N}_{4}$ result 12$)$ One side coated $\mathrm{Si}_{3} \mathrm{~N}_{4}$ deforms the wafer. At $1100^{\circ} \mathrm{C}$ there is still intrinsic stress in the $\mathrm{Si}_{3} \mathrm{~N}_{4}$ layer which causes the wafer to bend. At this high temperature the silicon substrate then plastically deforms. The deformation of silicon has been observed previously by Tamura et al [5]. Along the $<111>$ planes dislocations appear, changing the shape of the wafer permanently. These dislocations can be observed by looking at the surface of the silicon with a differential interference microscope. Figure D-II.a shows the surface of a deformed $<100>$ silicon wafer and Figure D-II.b shows a deformed $<110>$ silicon wafer. The lines that are visible in both pictures are along the $<110>$ 
direction, indicating dislocations along the $<111>$ planes. For the $<100>$ wafer this results in a squared pattern, as for the $<110>$ wafer it is a parallelogram.

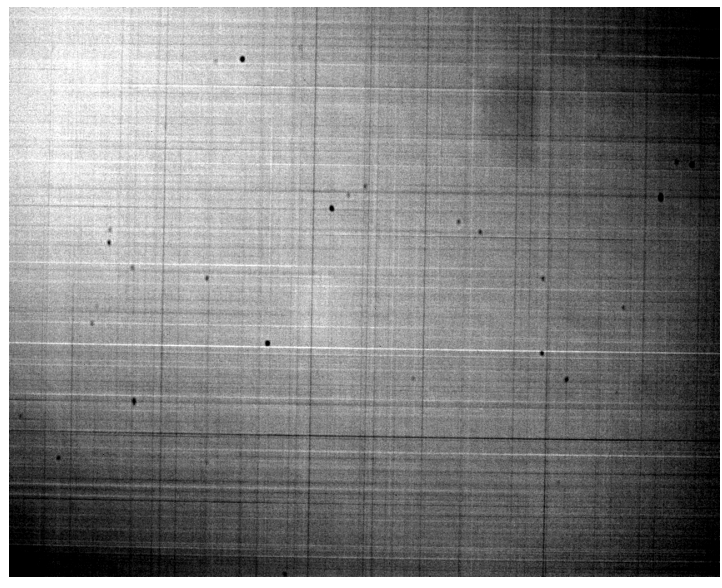

a)

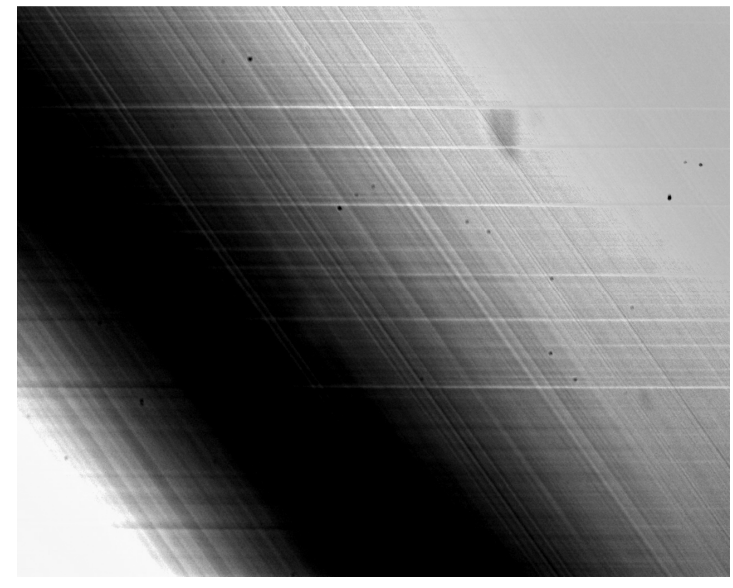

b)

Figure D-II: a) Dislocation lines on $a<100>$ silicon wafer b) Dislocation lines on a $<110>$ silicon wafer

The following conclusions are made from the D-series.

10. $\left(\boldsymbol{S i}_{3} \boldsymbol{N}_{4}\right.$ on $\boldsymbol{T E} \boldsymbol{O S}$ result 19$)$ The stress in $\mathrm{Si}_{3} \mathrm{~N}_{4}$ after 1 hour annealing at $1100^{\circ} \mathrm{C}$ of a both side coated wafer with $\mathrm{Si}_{3} \mathrm{~N}_{4}$ on top of TEOS reduces to $0.95 \pm 0.09$ GPa.

11. ( $\boldsymbol{S i}_{3} \boldsymbol{N}_{4}$ on $\boldsymbol{W O X}$ result 16 and 19) 15 hours annealing at $1100^{\circ} \mathrm{C}$ of a both side coated wafer with $\mathrm{Si}_{3} \mathrm{~N}_{4}$ on top of WOX reduces the $\mathrm{Si}_{3} \mathrm{~N}_{4}$ stress further to $0.77 \pm 0.09 \mathrm{GPa}$.

12. ( $\boldsymbol{S i}_{3} \mathbf{N}_{4}$ on $\boldsymbol{W O} \boldsymbol{X}$ result 16 and 17) A second time 15 hours annealing of $\mathrm{Si}_{3} \mathrm{~N}_{4}$ on top of WOX does not change the stress significantly anymore $(0.80 \pm 0.09 \mathrm{GPa})$. We conclude that after the first 15 hours the stress value is stabilized.

13. ( $\boldsymbol{S i}_{3} \mathbf{N}_{4}$ on WOX or TEOS result 18, 19 and 21) 15 hours annealing of one side coated $\mathrm{Si}_{3} \mathrm{~N}_{4}$ on top of WOX (result 18) and also for $\mathrm{Si}_{3} \mathrm{~N}_{4}$ on top of TEOS (result 21) results in practically no deformation of the silicon substrate. It is an asymmetrical system and we expected plastic deformation. We look now at wafer D5. At first this wafer is annealed for 1 hour with both sides coated. In this step a significant amount of stress is be released (result 19). We think that, if this wafer had been one side coated in this first hour of annealing it would have deformed. Plastic deformation of the silicon substrate happens in the first hour of annealing while the stress in the $\mathrm{Si}_{3} \mathrm{~N}_{4}$ is highest. Nevertheless, this result is in conflict with the literature which indicates that $\mathrm{Si}_{3} \mathrm{~N}_{4}$ has a smaller thermal 
expansion than silicon and thus, it should have bent the wafer with only one side coated. More experiments are needed to unravel this issue.

14. $\left(\boldsymbol{S i}_{3} \boldsymbol{N}_{4}\right.$ on WOX or TEOS result 14 and 16$)$ We patterned one side coated $\mathrm{Si}_{3} \mathrm{~N}_{4}$ on top of TEOS with squares of $1 \times 1 \mathrm{~mm}$ with $50 \mu \mathrm{m}$ spacing in between. This is done to see if this has an effect on the speed of the stress release. After 1+15 hours annealing the $\mathrm{Si}_{3} \mathrm{~N}_{4}$ stress lowers to $0.72 \pm 0.09 \mathrm{GPa}$ (result 14). Comparing this with 15 hours annealing of an $8 \mathrm{~cm}$ island of $\mathrm{Si}_{3} \mathrm{~N}_{4}$ on top of WOX (result $16,0.77 \pm 0.09 \mathrm{GPa}$ ) a small difference is observed but this is still in the error range. We conclude that the feature size of a floating island of $\mathrm{Si}_{3} \mathrm{~N}_{4}$ on top $\mathrm{SiO}_{2}$ is minor.

15. ( $\boldsymbol{S i}_{3} \mathrm{~N}_{4}$ on WOX or $\boldsymbol{T E O S}$ result 15$)$ One side coated $\mathrm{Si}_{3} \mathrm{~N}_{4}$ on top of TEOS and patterned with the squares- deforms the wafer strongly.

\section{The following conclusions are made from the S-series.}

16. ( $\boldsymbol{S i}_{3} \mathbf{N}_{4}$ on $\boldsymbol{W O X}$ or $\boldsymbol{T E O S}$ result $22,23,25$ and 26$)$ After $5^{*} 1+15$ hours annealing, the stress in the back $\mathrm{Si}_{3} \mathrm{~N}_{4}$ layer reduces to $0.69 \pm 0.09 \mathrm{GPa}$, which resembles the stress of the film on the frontside $(0.65 \pm 0.09 \mathrm{GPa})$. The same result is found for the $\mathrm{Si}_{3} \mathrm{~N}_{4}$ in WOX wafer $0.80 \pm 0.09$ and $0.73 \pm 0.09 \mathrm{GPa}$.

17. ( $\boldsymbol{S i}_{3} \boldsymbol{N}_{4}$ on WOX or TEOS result 24 and 27) Of a one side patterned wafer, the topside $\mathrm{Si}_{3} \mathrm{~N}_{4}$ layer is clamped around the wafer edges while the backside layer is free floating on top of TEOS or WOX. As already observed for the single side annealed $\mathrm{Si}_{3} \mathrm{~N}_{4}$ wafer, a one side patterned wafer also shows plastic deformation. An asymmetric system might result in bending of the substrate during the annealing and the wafer plastically deforms.

One additional comment is made regarding the experiments. It seems that the stress release in the $\mathrm{Si}_{3} \mathrm{~N}_{4}$ is faster when there is $\mathrm{SiO}_{2}$ underneath it. Nevertheless, after 15 hours of annealing the stress value of $\mathrm{Si}_{3} \mathrm{~N}_{4}$ goes to $0.7-0.8 \mathrm{GPa}$ independent of the basis it is deposited on.

From these experiments it is not yet clear how the stress changes in the membranes as presented in chapter 5. For the membranes a tensile stress in the $\mathrm{Si}_{3} \mathrm{~N}_{4}$ as low as $0.32 \pm 0.1 \mathrm{GPa}$ is found after 1 hour annealing at $1100^{\circ} \mathrm{C}$. Such a low stress value has not been measured for $\mathrm{Si}_{3} \mathrm{~N}_{4}$ on top of $\mathrm{SiO}_{2}$ with these experiments. Even after 15 hours annealing the lowest measured stress is about $0.72 \pm 0.09 \mathrm{GPa}$. However, 
there is a difference in the situation between the membranes and the experiments which might be the cause for the difference in this stress. For the experiments, the $\mathrm{Si}_{3} \mathrm{~N}_{4}$ on top of $\mathrm{SiO}_{2}$ is everywhere attached to the silicon substrate. Whereas, the membranes are free hanging and are only attached to the silicon substrates at the membrane edge. The membrane is pulling on the interface with the silicon substrate only locally. This results in a high stress concentration at this interface. At high temperature two things might happen. The membrane is attached to the silicon substrate by a $\mathrm{SiO}_{2}$ layer. This $\mathrm{SiO}_{2}$ layer might slip at high temperature due to the lowered viscosity and the pulling of the $\mathrm{Si}_{3} \mathrm{~N}_{4}$ film. The second possibility is plastic deformation of the silicon substrate at the interface with the membrane at high temperature. We think this latter possibility is less probable because we were able to make tensile membranes by clamping them to the silicon substrate. If the substrate would have locally deformed the membranes should have buckled. More experiments are required to solve this problem.

[1] Stoney G. G., The Tension of Metallic Films Deposited by Electrolysis, Proc. Roy. Soc. London Ser. A 82 (1909), p. 172.

[2] Pister K.S. and Dong S.B., Proceedings Am. Soc. Civil Eng. 85 (1959).

[3] EerNisse E.P., Viscous flow of thermal $\mathrm{SiO}_{2}$, Appl. Phys. Lett. 30 (1977).

[4] Touloukian Y.S., Thermophysical Properties of Matter, IFI/Plenum, New York (1977).

[5] Tamura M. and Sunami H., Generation of Dislocations Induced by Chemical Vapor Deposited Si3N4 Films on Silicon, Japan. J. Appl. Phys. 12 (1972), pp. 1097-1105. 


\section{Appendix E - TEOS sponge effect}

Just after deposition of a TEOS layer by chemical vapor deposition the stress is $10 \mathrm{MPa}$ tensile. Within a couple of days after the deposition this layer tends to become compressive as is shown in Figure E-I. When the TEOS film is annealed at $750^{\circ} \mathrm{C}$ for 1 hour it becomes tensile as is also shown in Figure. It is postulated that at $750^{\circ} \mathrm{C}$ all water is removed from the TEOS layer. When exposed to ambient conditions, water vapor is absorbed and the film becomes compressive; the TEOS film acts as a sponge. When the TEOS film is covered with poly silicon or silicon nitride - directly after TEOS deposition - the layer stays tensile. Water vapor is unable to diffuse through the poly silicon or silicon nitride and thus the sponge effect is inhibited.

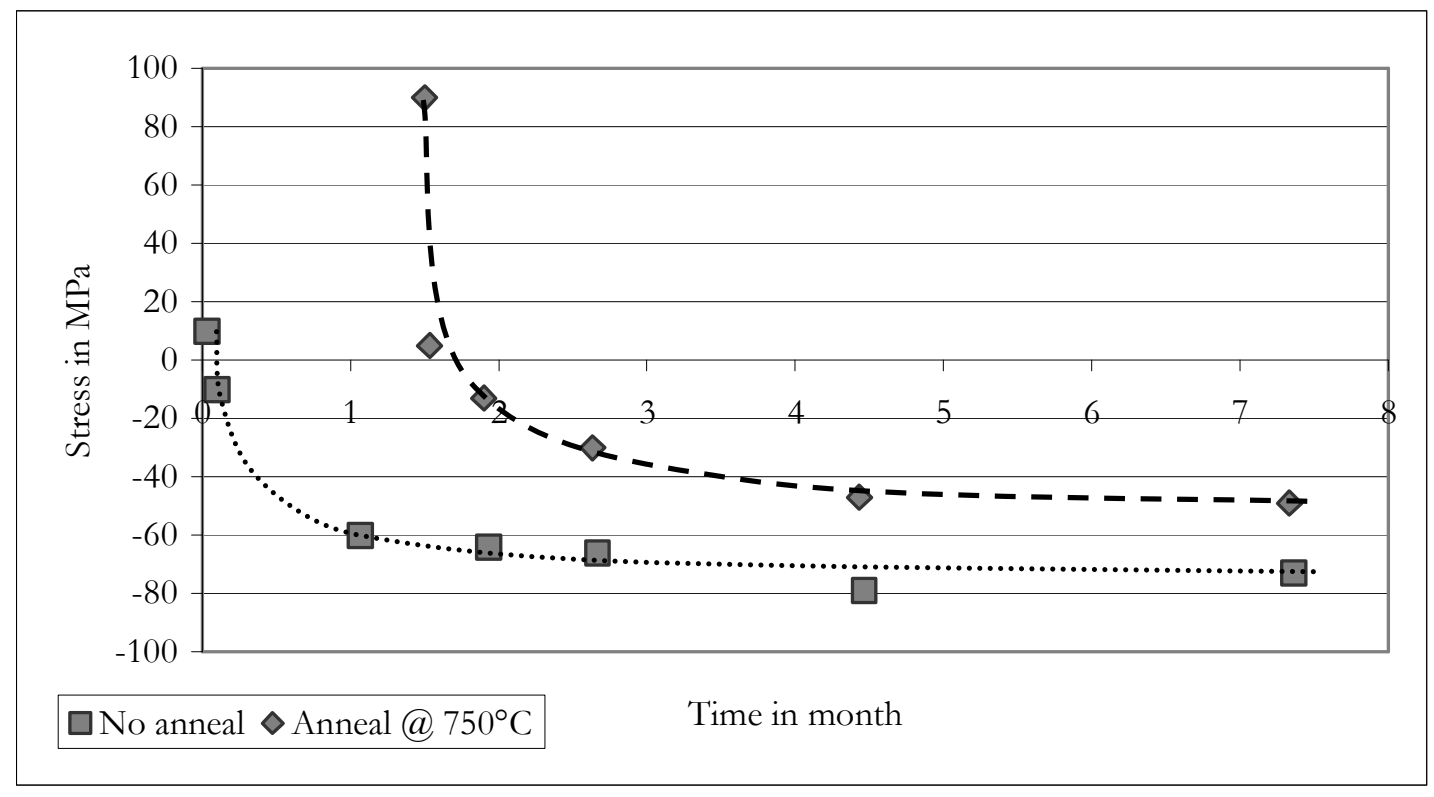

Figure $\boldsymbol{E}$-I: Stress in TEOS as a function of time after deposition 


\section{Summary}

This thesis describes the development of a micro propulsion system. The trend of miniaturization of satellites requires small sized propulsion systems. For particular missions it is important to maintain an accurate distance between multiple satellites. Satellites can drift apart due to differences in mass, area and position of the satellites. Design guidelines have been derived for a propulsion system which is supposed to control the distance between two small satellites. The design guidelines show that the dimensions of several system components are in the micrometer range which makes micro system technology (MST) a suitable option.

A miniaturized propulsion system of a cold gas blow-down rocket engine is presented. The system consists of two parts; the low pressure storage system and the feeding and thruster system. The feeding and thruster system can generate the thrust required for position corrections of the satellite. It consists of several functional modules; an active valve, a particle filter, a nozzle and an electronic module. A modular platform is presented to put these functional modules together. The modules are adjusted to fit in a baseline package. This package functions as a fluidic interconnection and a macro support for the functional modules. The nozzle and the valve are the key components of the system and are researched in more detail.

A nozzle is needed which can deliver a thrust in the $\mathrm{mN}$ range. The nozzle is used to increase the efficiency of the propulsion system. We explored the fabrication of a 3D conical converging-diverging nozzle shape by means of MST techniques. These techniques are: deep reactive ion etching, femtosecond laser machining and a technique which uses powder blasting in combination with a heat treatment. It is shown that the latter two methods are most promising.

The valve has to be very leak-tight to ensure a successful satellite mission. In conventional valves a low leak-rate is obtained by a large and forceful actuator. To allow for smaller valves a novel design is presented requiring low force - and thus a smaller actuator - while still promising to be leak-tight. A flexible thin film 
membrane is pulled over a valve seat pattern to make a leak-tight seal. Due to the flexibility of this membrane it can deform and adjust to the surface topography. From technological point of view, a lot of progress has been made. However, further research is necessary to be able to manufacture a fully functioning valve. 


\section{Samenvatting}

In dit proefschrift wordt de ontwikkeling van een micro voortstuwingssysteem beschreven. De trend van het miniaturiseren van satellieten vereist een klein voortstuwingssystemen. Voor bepaalde missies is het belangrijk dat de afstand tussen meerdere satellieten accuraat wordt gecontroleerd. Satellieten kunnen uit elkaar drijven door verschillen in massa, oppervlak en door verschilde omgevings omstandigheden. Enkele ontwerprichtlijnen zijn opgesteld voor een voortstuwingssysteem dat bedoeld is om de afstand tussen twee satellieten constant te houden. Hieruit blijkt dat de dimensies voor verschillende componenten van het systeem in het micrometer gebied liggen. Voor de ontwikkeling van componenten met dit soort afmetingen is het gebruik van micro systeem technologie (MST) een interresante optie.

Een geminiaturiseerd voortstuwingssysteem gebaseerd op een koud gas 'blowdown' raket motor wordt gepresenteerd. Het systeem bestaat uit twee delen; Het lage druk gas opslag systeem en het voedings- en stuwkrachtssysteem. Het voedings- en stuwkrachtssysteem kan een nauwkeurige voortstuwing leveren om precieze positie correcties van de satelliet te kunnen uitvoeren. Dit systeem bestaat uit een aantal functionele modules; een actieve klep, een deeltjes filter, de nozzle en een electronica module. Een modulair platvorm wordt gepresenteerd om de verschillende modules aan elkaar te koppelen. De modules worden zo ontworpen dat ze in een standaard behuizing kunnen worden geplaatst. Deze behuizing functioneerd als een gas aansluiting en een macro houvast voor de fragiele modules. De nozzle en de klep zijn de belangrijkste componenten van het systeem en zijn in meer detail onderzocht.

De nozzle moet een stuwkracht kunnen leveren in het $\mathrm{mN}$ bereik. De nozzle wordt gebruikt om de efficientie van het voortstuwingssysteem te verbeteren. De fabricage, door middel van MST technieken, van een 3D conische convergerendedivergerende nozzle vorm is onderzocht. Deze technieken zijn: reactief ionen etsen, femtoseconde laser fabricage en een techniek die gebruik maakt van poederstralen 
in combinatie met een hitte behandeling. De laatste twee methoden zijn het meest veel belovend.

De klep moet erg lek dicht zijn om een succesvolle satelliet missie te garanderen. Bij conventionele kleppen wordt een lage lek bewerkstelligd door een grote en krachtige actuator. Een nieuw klep ontwerp wordt gepresenteerd waarvoor weinig kracht nodig is, en dus een kleinere actuator, terwijl de lek toch erg laag beloofd te zijn. Een flexibel membraan, van dunne film materiaal, wordt over een klepzitting patroon heen getrokken om een goede afdichting te maken. Door de flexibiliteit van dit membraan kan het zich vervormen naar de oppervlakte topografie van de klepzitting. Vanuit technologisch oogpunt is er flinke vordering gemaakt. Desondanks, is er meer onderzoek nodig om een volledig werkende klep te maken. 


\section{Dankwoord}

Op deze plaats wil ik graag een aantal mensen bedanken die de afgelopen vier jaar, op wat voor manier dan ook, hebben bijgedragen aan het tot stand komen van dit proefschrift.

Als eerste wil ik Miko bedanken, zonder promotor ook geen promotie! We hebben niet heel veel contact gehad gedurende de afgelopen vier jaar. De momenten dat we elkaar spraken zijn toch vaak geruststellend geweest; het komt allemaal goed.

Henri, we hebben intensief samengewerkt en ik heb dat erg prettig gevonden. Ik vind je een fijn persoon! Luttele ets-uurtjes hebben we met Dixy doorgebracht en toch moesten we de resultaten softwarematig in elkaar draaien. De stress experimenten, die op de valreep moesten plaatsvinden, waren uiteindelijk 'relaxerend'.

Job wil ik bedanken voor zijn bijdrage aan het onderzoek naar de nozzle fabricage. Ik hoop dat ik niet al teveel een beroep op je hoef te doen tijdens de verdediging! Veel belangrijker dan je wetenschappelijke bijdrage vind ik onze uitwisseling van gedachten over het leven waarbij we elkaar precies begrijpen en vaak maar een half woord nodig hebben.

Dan de kamer Meint-Erwin; hangen over het cubical muurtje en ouwehoeren, fijn! Erwin: ik ben je dankbaar dat je in de laatste maanden nog een poging hebt gedaan om een klep uit de Cleanroom te krijgen. Daar zijn toch weer belangrijke resultaten uit gekomen.

Ik heb genoten van de koffie-lunch-etenjes met: Pino, Dennis, Doekle, Jeroen en aanhang. Soms drinken we echt te lang koffie/thee... Alle overige MicMeccers: Bedankt! voor de prettige sfeer die in de groep aanwezig is.

Als laatste, Guillaume et Josephine (natuurpark le Treillut): Heerlijk om een plek te hebben waar je altijd liefdevol wordt ontvangen en er even helemaal tussenuit kunt om daarna weer fris en fruitig verder te kunnen. 
